\title{
LITHIC ANALYSIS OF THE MIDDLE AND LATE UPPER PALAEOLITHIC IN HUNGARY
}

\author{
GYÖRGY LENGYEL
}

A u th o r's a d d re s s: Institute of Systematics and Evolution of Animals, Polish Academy of Sciences, Sławkowska 17, 31-016 Kraków, Poland, e-mail: lengyel.isea@gmail.com

A b s t r a c t. This paper presents lithic technology studies on the Middle and Late period of the Upper Palaeolithic in Hungary between 26 and $13 \mathrm{ka}$ BP. The studies aimed at describing and then comparing the technological processes from lithic raw material procurement to the formal tool making. An attempt was made to find correlations between technological features and chronological positions of the assemblages to see if lithic technology operated traditionally or opportunistically. The study found that technology was rather shaped toward efficiency with an adaptive behavior. Therefore, in most cases, the way how tools were made is useless to differentiate archaeological cultures, while the tools themselves, especially the armatures, can be markers of cultures as was shown earlier. This study found that the formation of the archaeological record and its variability most likely depended upon the dynamism of human ecology.

K e y w o r d s: Lithic technology, technical behavior, raw material procurement, hunter-gatherers, subsistence strategy

\section{INTRODUCTION}

The archaeological record of Late Pleistocene modern humans in Hungary is chiefly represented from the Middle and Late Upper Palaeolithic (MUP and LUP) periods 28-12 ka BP. MUP and LUP lithic assemblages had long been classified with the Gravettian Entity model (GEM) into four discrete units of the Gravettian family: Pavlovian 28-26 ka BP, Ságvárian 20-17 ka BP, Epigravettian (the "expedient") 18-16 ka BP and Epigravettian ("rich in blunted blades") 16-12 ka BP (DoBosi 2000a, 2004, 2009a, b). GEM claimed that Pavlovian tools were always made with higher quality, but originated the Epigravettian in the Pavlovian, and meanwhile isolated the Ságvárian being different on the basis of technological characters (DoBosi 2004, 2009b). GEM's chronology thus was based upon lithic tool typology, radiocarbon dates and lithic technology.

A test on the typological considerations of GEM found that: 1) the units are typologically different, 2) the Pavlovian term cannot be applied for the Hungarian assemblages 
because Pavlovian tools are missing and the finds of this unit possess Late Gravettian characters dated to $25-21 \mathrm{ka} \mathrm{BP}$ elsewhere, 3) in fact, there is no convincing data on Gravettian occupation before $26 \mathrm{ka} \mathrm{BP}$, and 4) the Ságvárian typologically is not different from the "expedient" Epigravettian (Lengyel 2016). The revised typology thus suggested an alternative chronology for the MUP and LUP in the Carpathian basin, which consisted of Late Gravettian (LG) 26-21 ka BP, Early Epigravettian (EE) 21-17 ka BP and Late Epigravettian (LE) 17-13 ka BP human occupations (LENGYEL 2016). This alternative classification was largely supported by the revised radiocarbon database of GEM (LENGYEL 2008-2009).

GEM's taxonomy also was laid on lithic technological observations and assumed that lithic assemblages produced by the same brand of technology likely had a common cultural origin. This was emphasized with an alternative vocabulary in GEM, which termed Older Blade Gravettian, Pebble Gravettian and Younger Blade Gravettian the Pavlovian, Ságvárian and the Epigravettian, respectively (Doвosi 2004). The supporting technological information emerged from the distinction between blade and flake production on pebble or not pebble raw material. As a result, the presence of a classic slender blade technology supported to originate the Epigravettian in the Pavlovian along the Blade Gravettian lineage. The Ságvárian that produced mainly flakes and short blades was an outlier of the blade technology heritage, and its pebble economy gave it an atypical character rooted probably in the pebble user Middle Palaeolithic (Tolna-Dobosi 2001; Dobosi 2004, 2009b, 2016).

To confront basic lithic technology data, as GEM has done that, to define cultural entities, must be handled with care because the flintknapping process and the subsequent archaeological record often could have been affected by the availability, quality and shape of the raw materials (ANDREFSKY 1994a, b; LENGYel 2013; TIXIER 2012). On the other hand, there are more modalities even within the domain of blade technology which can make lithic assemblages different (PeLEGRIN 2011), and undeniably there is a relation between human subsistence strategy and lithic assemblage formation (BINFORD 1979, 1982; Kelly 1983, 2013; ANDREFSKY 2009).

Therefore, involving the lithic technological component among the principles of GEM brings up several aspects of the formation of the MUP and LUP lithic archaeological record in Hungary which are yet unclear. If indeed there was a technological lineage from the Gravettian to the Epigravettian, there must be clear traces of it, inferring to similar ecology, subsistence strategy and technological knowledge, which could be detected with an analysis digging deeper into the domain of lithic technology.

\section{THE MUP AND LUP IN EASTERN CENTRAL EUROPE}

During the hundreds of millennia of the Pleistocene, the fluctuation of the terrestrial ice sheets in the northern hemisphere profoundly affected the settlements of humans (TAllavaARaA et al. 2015). Well-known cold period in European prehistory is the Upper Pleniglacial, in which the continental ice sheet restarted southward advancing 
after a mild period of the Weichselian Interpleniglacial (Boulton et al. 2004). This cooling peaked in the Last Glacial Maximum (LGM), which according to global data earliest started at $\sim 24 \mathrm{ka} \mathrm{BP}$ and lasted until $17 \mathrm{ka}$ BP (ClARK et al. 2009; Hughes et al. 2013). The LGM in Eurasia united the British-Irish, Fennoscandian and BarentsKara ice sheets into a Eurasian Ice Sheet (EIS) (Grosswald 1980). EIS reached its maximum extent by $21 \mathrm{ka}$ BP with covering seven million square kilometers from the British Isles to the Taymyr Peninsula between the $52^{\circ} \mathrm{N}$ and $77^{\circ} \mathrm{N}$ latitude (Hughes et al. 2016). The EIS lasted in this position until $18 \mathrm{ka} \mathrm{BP}$ and began retreating during $17 \mathrm{ka}$ BP. By $12 \mathrm{ka}$ BP, the line of the current Baltic sea shore line was once again unglaciated (MARKs 2012; STROEVEN et al. 2015).

The MUP and LUP human record in eastern Central Europe (ECE) consists of three archaeological cultures that represent three hunter-gatherer populations: Gravettian 30-28 ka BP, Epigravettian 20-13 kya BP and Magdalénian 15-12 kyr BP (SvoBODA 2007; MAIER 2015; LENGYEL, WILCZYŃSKI 2018). The Gravettian archaeological record further can be classified into three clusters. The earliest is the Early Gravettian, dated to $\sim 30-28 \mathrm{ka} \mathrm{BP}$ (Moreau 2009). The next is the Pavlovian, dated to $\sim 27-26 \mathrm{ka}$ BP (Svoboda 2016). The last member of the Gravettian culture is the Late Gravettian, also called Willendorf-Kostenkian (Svoвoda 2007), which occupied ECE between 26-20 ka BP (WILCZYŃSKI 2016).

Gravettian lithic tools are distinct from the subsequent periods. One key to classify ECE lithic assemblages is the armature component of the retouched tool kit (LENGYEL 2016). Early Gravettian armatures are the microgravette, backed bladelet, retouched blade point and fléchette (Moreau 2009). The Pavlovian contains the Early Gravettian armatures, and additional tools are the backed denticulated bladelets, crescents, triangles and basally inverse thinned blade points (KozŁowsKi 1996, 2015; SvoBodA 1996, 2007). The Late Gravettian lacks these additional Pavlovian types, but besides the Early Gravettian armatures, it further has shouldered point, backed truncated bladelet, rectangle, including backed ventrally truncated type and sometimes bifacial tools (LeNGYel et al. 2016). The Early Epigravettian, previously often called Ságvárian (KozŁowski 1979) or Kašovian (SvobodA, Novák 2004), completely lacks the Gravettian armature except for the backed bladelet, sometimes truncated too, and the retouched point. The Late Epigravettian armature includes an abundant number of backed armature, including straight, curved and arched backed points, rectangles and backed-truncated bladelets. The Magdalenian armature also consists of backed bladelets, which are sometimes truncated, but interestingly, its key lithic tool type in ECE is a domestic tool, the Lacan type burin and the borer (PoŁтоwicz-BoвAK 2012).

According to the distribution of armature types and radiocarbon dates in the MUP and LUP in ECE, Early Gravettian occupations are scarce and the only firm archaeological record is located at Willendorf II in layer 5 (MorEAU 2012) (Fig. 1). Henryków 15, southwestern Poland, was also dated to this period (WIŚNIEWSKI et al. 2015), but it differs from Willendorf II layer 5 because the microgravette is absent and there is a bifacially shaped point that is not characteristic to Early Gravettian inventories (Moreau 2009). Within the Carpathian basin, the only human occupation in this period 


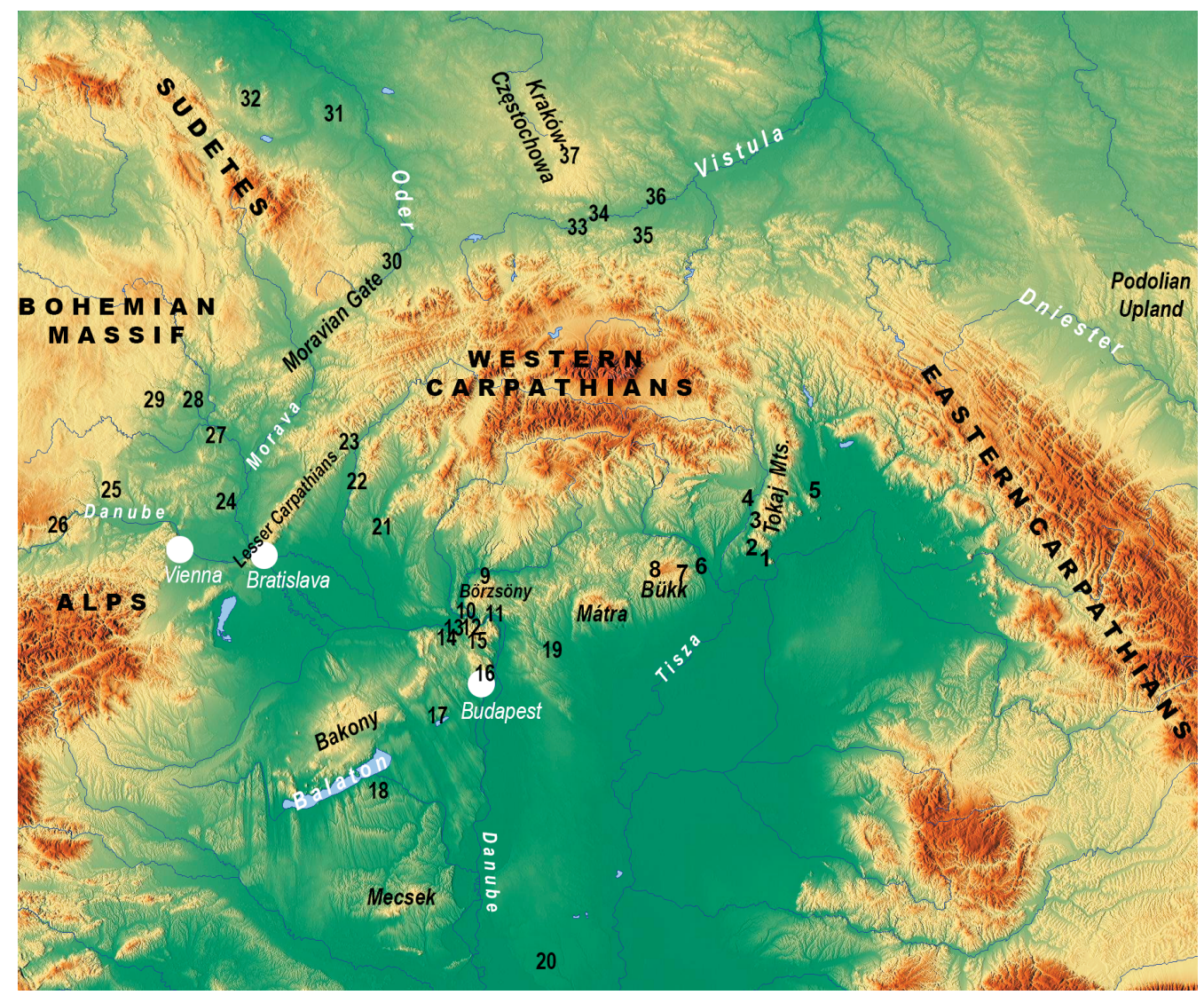

Fig. 1. Major sites mentioned in the text. 1 - Bodrogkeresztúr; 2 - Megyaszó; 3 -Arka; 4 - Hidasnémeti; 5 - Kašov I, Cejkov I; 6 - Sajószentpéter; 7 - Szeleta cave; 8 - Istállóskő Cave; 9 - Hont-Parassa III; 10 - Szob; 11 - Verőce; 12 - Pilismarót; 13 - Esztergom; 14 - Mogyorósbánya; 15 - Pilisszántó I rockshelter; 16 - Budapest-Csillaghegy; 17 - Nadap; 18 - Ságvár; 19 - Jászfelsőszentgyörgy; 20 - Madaras; 21 - Nitra-I Cěrmáň; 22 - Moravany sites; 23 - Trenčianske Bohuslavice; 24 - Grub-Kranawetberg; 25 - Grubgraben; 26 - Willendorf II; 27 - Dolní Věstonice, Pavlov, Milovice I; 28 - Brno Štýřice III; Stránská skála IV; 29 - Mohelno-Plevovce; 30 - Petřkovice I; 31 - Sowin 7; 32 - Henryków; 33 - Piekary II; 34 - Kraków-Spadzista; 35 - Targowisko 10; 36 - Jaksice II; 36 - Deszczowa Cave

is in Istállóskő cave upper layer (Aurignacian II in VÉRTES 1955) in Bükk Mountains, Northeast Hungary, which yielded Mladeč point and a mixed character of lithics (Davies, Hedges 2008-2009; Markó 2015; Patou-Mathis et al. 2016).

After the Early Gravettian, between 28 and 26 ka BP, Pavlovian occupations are found solely in Moravia (Dolní Věstonice and Pavlov sites) (Svoboda 2007) and Lower Austria (HäNDEL 2017). Parallel with the disappearance of the Pavlovian archaeological record in Moravia and Lower Austria, Late Gravettian lithics started overtaking the archaeological record between 26 and 21 ka BP (LENGYEL, WILCZYŃsKI 2018).

In contrast to the preceding Gravettian periods, the Late Gravettian was widely spread from the western edge of the Middle Danube basin to the Dniester basin, 
including the Carpathian basin (KozŁowsKi 2008). Most prominent sites in ECE are Kraków-Spadzista in Poland (KozŁowski, SobczyK 1987; WiLczyŃski 2016), Petřkovice I (NováK 2008) and Milovice I (Oliva 2009) in the Czech Republic, Willendorf II layer 9 (Otте 1981) in Lower Austria, Trenčianske Bohuslavice (BÁRTA 1988), Moravany-Banka (KozŁowski 2000) and Nitra-I Cěrmáň (KamiŃsKA, KozŁowsKi 2011) in Slovakia. The revised Hungarian MUP and LUP chronology (Lengyel 2015, 2016) showed that sites called Pavlovian by GEM such as Bodrogkeresztúr, Hidasnémeti and Sajószentpéter, suit Late Gravettian taxonomy. The lithic assemblage of Arka, formerly classified Epigravettian, showed strong similarity with Bodrogkeresztúr. Further Late Gravettian armature can be found in Pilisszántó I rock shelter (DoBosI, Vörös 1987; Kormos, Lambrecht 1915), Szeleta Cave layer 6 and 5 (SimÁn 1990; Lengyel et al. 2016) and Hont-Parassa III (Dobosi, Simán 2003).

With the onset of the LGM, human settlements seem to have disappeared from the north of the Carpathians and the Sudetes, thus Early Epigravettian lithics mostly are found in Moravia, Lower Austria and in the Carpathian basin. In Hungary, taking into account the armature types and their frequencies, Ságvár, Budapest Corvin-tér (LENGYEL 2016), the Pilismarót site cluster (Pálrét, Diós, Bitóc and Bánom) (DoBosi 2006, 2014), Jászfelsőszentgyörgy (DoBosi 2001), Szob (Markó 2007), Madaras (Dobosi et al. 1989), Mogyorósbánya (DoBosi 2016), Herman Ottó Cave (Szolyák 2008-2009), Veröce (BRADÁK et al. 2014) and Tarcal-Citrombánya (Dobosi 1974) can be listed here. Sites of this period outside Hungary are Kašov upper layer (BÁNESZ et al. 1992) in East Slovakia, Grubgraben (Montet-White 1990; Neugebauer-Maresch et al. 2016) in Lower Austria and Stránská skála IV (SvobodA 1991; ŠKrdLA, PlCH 1993) and Mohelno-Plevovce (Demidenko et al. 2018) in Moravia. Often mentioned Epigravettian sites in Poland are Piekary IIa layer 5 and Kraków-Spadzista layer 5 in B+B1 (WILCZYŃSKI 2007; WiśNIEWSKI et al. 2017). Kraków-Spadzista C2 area layer 6a yielded a single radiocarbon date of LGM age 17.4 ka BP (KozŁowski, SoBCZYK 1987), but this is clearly associated with Late Gravettian lithics and re-dated to 22-24 ka BP (WILCZYŃSKI et al. 2015). Layer 5 remained undated, and all we know about it is that it postdates the Late Gravettian with unknown gap of time. Deszczowa Cave layer VIII in the Kraków-Częstochowa Upland northwest of Kraków yielded a few artifacts including a retouched blade and end scraper dated to between 17.4 and 20.8 ka BP (CYreK et al. 2000; NADACHOWSKI et al. 2009), but the overlying layers IX and X were dated to 22-24 ka BP (LorENC 2006), which warns for caution interpreting these findings. Therefore, Poland likely was deserted during the LGM.

When EIS started retreating, Late Epigravettian hunters repopulated the north of the Carpathians. Two important sites in Hungary, which are the only ones in the viewpoint of typology, are Esztergom and Nadap. Latter formerly was classified Pavlovian (DoBOSI 2009a). Uncertainly, Megyaszó site classified among "Pavlovian" (DoBosi, SimáN 1996) could also be listed here, whose armature consists of mostly backed bladelets and points without Gravettian character. Budapest-Csillaghegy (GÁBORI-CsáNK 1986), dated to 15.9 ka BP (SüMegi et al. 1998; Lengyel 2008-2009), seems to be a human occupation of the deglaciation period, however, it does not have Late Epigravettian 
armature. Sites outside Hungary, Targowisko 10 (WILCZYŃSKi 2009), Sowin 7 lower layer (WiŚNIEwSKI et al. 2012, 2017) and Brno-Štýřice III (NERUdová, NERUdA 2014) were dated to $c a$. $15 \mathrm{ka} \mathrm{BP}$.

The Magdalenian occupation of ECE occurred with the late phase of this culture, but Magdalénian hunters did contribute to the formation of the archaeological record in the Carpathian basin (MaIER 2015).

\section{METHODS}

The archaeology of human relics from the Pleistocene significantly differs from later periods of the Prehistory. The time between the burial of the finds and their unearthing often faced sever geological processes that altered the Pleistocene landscape and eventually affected the preservation of the finds. Artifacts made of organic materials easily decay in the ground due to physical and chemical effects. But, stones used to make tools for scraping, cutting, carving, engraving and hunting weaponry were resistant to most geochemical and physical processes in the soil. The durability of the stone is the reason for knapped lithic tools to be the most common archaeological find material at Pleistocene hunter-gatherer camps.

Stone tools, therefore, are found wherever hunters established camps. The lithic tools at hunter-gatherer camps are two sorts. One sort of tools was used to make the equipment, clothing, sheltering, butchering, to accomplish domestic activities. The other sort of tools is the hunting weaponry. It is up to the function of the camp which type of tool is found in majority. A residential camp contains many of the domestic tools because it is the place to host the group for a longer period, while a field camp devoted for hunting is likely to yield a wealth of armature lithic types which were abandoned after damage and replaced with new items (BINFORD 1980, 1982, 1990).

To subsist as a hunter-gatherer, foraging for animal and vegetal food is vital, which needs coordinating apt techniques, knowledge and the technological processes (SнотT 1986; Kelly 2013). In arid environment, alike the Pleniglacial Europe, the subsistence was regularly based upon hunting because vegetal resources might be scarce, and migratory preys subsequently made humans mobile (PrYOR 2008; VerPoORTE 2009; Kelly 2013). Thus, mobility can distribute the archaeological record over vast areas.

A general proxy to recognize human mobility is the lithic raw material provenience (GoODYEAR 1979). Since raw material procurement often can be embedded within hunting or food gathering activities (BINFORD 1979), the distance between site and lithic source may mark the annual range of hunter-gatherers (BINFORD 1982). In eastern Central Europe, there is great potential for mapping Upper Palaeolithic mobility patterns (FÉBLOT-Augustins 1997), because siliceous rock raw materials are highly variable around Carpathians due to being regionally specific (P̌̌ICHYSTAL 2013). Therefore, studying the lithic technology generates issues of subsistence strategies.

The lithic technology study performed here embraces the production process from lithic raw material procurement to tool making. The lithic raw material provenience 
classification made the use of the Lithoteca collection at the Hungarian National Museum (BIRó, Dobosi 1991; Biró et al. 2000) and the Lithic Reference Collection of the ELTE University of Budapest (Mester 2013).

The lithic raw materials were divided into three categories according to their origin. Local materials were derived from within $10 \mathrm{~km}$ radius of a site. This distance was simply defined by time considerations, which means that the raw material can be collected and brought to the site within one day. This correspond with the 6 miles foraging radius of BINFORD (1982) and also assumes that the site location was not randomly chosen but to locate to maximize foraging efficiency (VITA-FINZI et al. 1970). Beyond the local zone was the regional one between 10 and $100 \mathrm{~km}$ from the site. This would fairly correspond with the logistical radius of a hunter-gatherer residential site (BINFORD 1982). Distant raw materials were those located over $100 \mathrm{~km}$ but within the Carpathian basin. Using BINFORD's (1982) zonation, this can be equal to the extended range. The fourth zone is called here transcarpathian (TC) territory that includes lithic sources beyond the arch of the Carpathians, and may fit the visiting zone of BINFORD (1982).

Each lithic assemblage was divided into eight technological categories: flakes, blades, debris, platform rejuvenating flakes of blade cores (core tablets included), crest blades, neo-crest blades, blade cores and flake cores following InIZAN et al (1999). Contrary to what has been applied widely in lithic technology, blades and bladelets were undifferentiated, and the term "blade" covers all the laminar products regardless their size.

The lithic raw materials were weighed in grams by major technological groups: blades, flakes, debris and cores.

The lithic technology study aimed at revealing the debitage(s) applied. The term debitage here means the flintknapping process that aims at producing blank flakes or blades for tools from a piece of lithic raw material via reducing a core (INIZAN et al. 1999). Each debitage is presented from raw material procurement to tool retouching. This was based on the concept of the chaine opératoire (INIZAN et al. 1999). Since blade debitage was found in each assemblage, the production of blades is always separated from that of the flakes. The parameters of the flakes were presented even if no flake debitage was found, because they were regarded as the by-products of the blade debitage.

The blade debitage was divided into two chief core reduction methods: unidirectional and bidirectional. These were traced by the orientation of the scars on the dorsal faces of the blades. Scar orientation parallel with the orientation of the blade was defined unidirectional, while oppositely patterned scars represented bidirectional debitage. Also, the number of the striking platform on the cores were used to define the orientation of the debitage. Single striking platform represented the unidirectional method, and opposed platformed cores were evidences of bidirectional debitage core reduction. A specific mode of the debitage that applies two opposed striking platforms is when the debitage surfaces do not cross each others' way; this is called alternate debitage. In this case the blades have unidirectional scars on their ventral faces. The same considerations were used for the flake debitage. Additionally, a third specific debitage method that used several sides of the core to remove flakes and resulted in multiple platforms was differentiated. 
The knapping technique was also defined in the debitages. The knapping technique, direct soft and hard hammer percussions, herein does not mean the matter of the hammer as the names of the techniques refer to it (InIZAN et al. 1999). Instead, these are understood as the movement of the knapper's arm holding the hammer. Therefore, direct soft hammer percussion may include any hammer type that tangentially hit the edge of the core. Delivering the strike onto the surface of the striking platform is the direct hard hammer percussion technique. Soft hammer technique was defined when the overhang was abraded and the impact point was missing. Hard hammer technique was defined when the impact point was present and the overhang remained unabraded. When impact point is present and the overhang is abraded, there is a chance that the tangential edge hit was inaccurate and impacted surface of the striking platform. When the overhang remained unabraded and impact point did not occur, the technique was classified hard hammer because of the lack of platform edge abrasion, which means that the platform edge was not prepared for soft hammer technique.

The flake and the blade platforms were classed into plain, dihedral, faceted, cortical, punctiform, linear and irregular types. Platform type was used to investigate the preparation of the core striking platform before removal.

The lithic artefact size was recorded with three measurements following INIZAN et al. (1999). Length was measured along the technological axis of the artifact. Thickness was measured at the greatest distance between the dorsal and the ventral face perpendicularly to the plan of the artifact. Width was measured between the left and the right edges of the artifact perpendicularly to the technological axis.

The technological data recording was accomplished solely on complete unretouched artifacts. Broken and retouched artifacts were excluded because the missing parts could have borne vital technological information to classify the given artifact. These artifacts, however, were added to the counts of the technological classes of the lithic assemblages. Retouched artifacts were measured for length, width and thickness.

The length, width and thickness measurements are presented by the minimum and the maximum values, the mean, the median and the standard deviation.

The technological data were broken down by raw material types to find differences in the processing of the different raw materials. To compare two sets of measurements, t-test was applied. Comparing more than two sets of data, ANOVA was used with Tukey post hoc test.

A lithic tool means any lithic artifact whose original edges were modified by retouching or burin spall removal. Studied were the selection of blanks for tools, the ratio of the blank types among the tools, the frequency of the given blank type, for instance blades, were used up to make tools.

The lithic tools were divided into two major groups, armatures and domestic tools. Armature includes tools which were parts of a hunting weaponry (ELSTON, BRANTIGHAM 2002). Armatures are the backed and backed-truncated blades and the points (Fig. 2).

The criterion for backing was restricted for those pieces whose edges were perpendicularly retouched up to the thickest part of the blank. If the perpendicular retouch did not reach the thickest part of the blank, and the retouch was visible only on the 


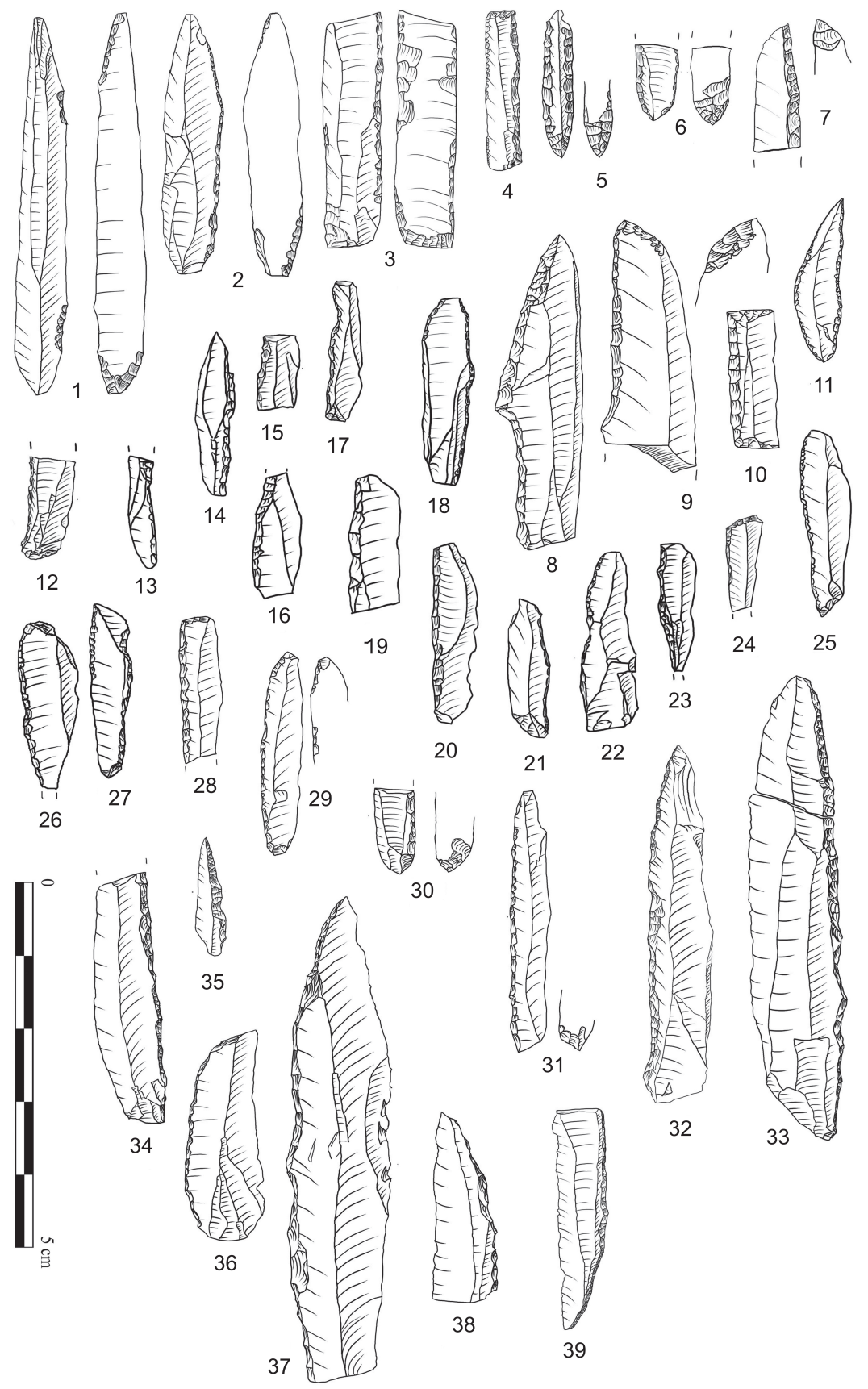

Fig. 2. Armatures typology of the studied sites. 1-7 - Bodrogkeresztúr; 8-11 - Hidasnémeti; 12-24 - Ságvár; 25, 26 - Corvin-tér; 27-31 - Arka; 32-35 - Nadap; 36-39 - Esztergom. Fléchette: 1, 2, 11, 29; Backed ventrally truncated blade: 3; Backed-truncated blade: 4, 24, 28; Vachons point: 5, 6; Gravette/microgravette: 7, 9, 30, 31; Retouched point: 25; Abruptly retouched blade: 18, 21; Shouldered point: 8; Rectangle: 10; Backed blade: 12-17, 19, 20, 22, 23, 26, 27, 34; Curved backed point: 32, 37-39; Arched backed point: 36; 
edge, the piece was classified as an abruptly retouched tool and sorted into the edge retouched tools.

Points were divided into four classes. Retouched points were made with regular edge retouch at the tip of the blank. The Gravette/microgravette point must have a basal inverse flat retouch opposed to the backed edge (Demars, LAURENT 1992). Rarely, the inverse retouch might have occurred on the distal part. When a point was backed without further retouching on the opposed edge, it was simply classified backed point. The delineation of the backed edge served to refine the variants of backed point types. Backed blade with straight back ending in a point were backed points. Backed

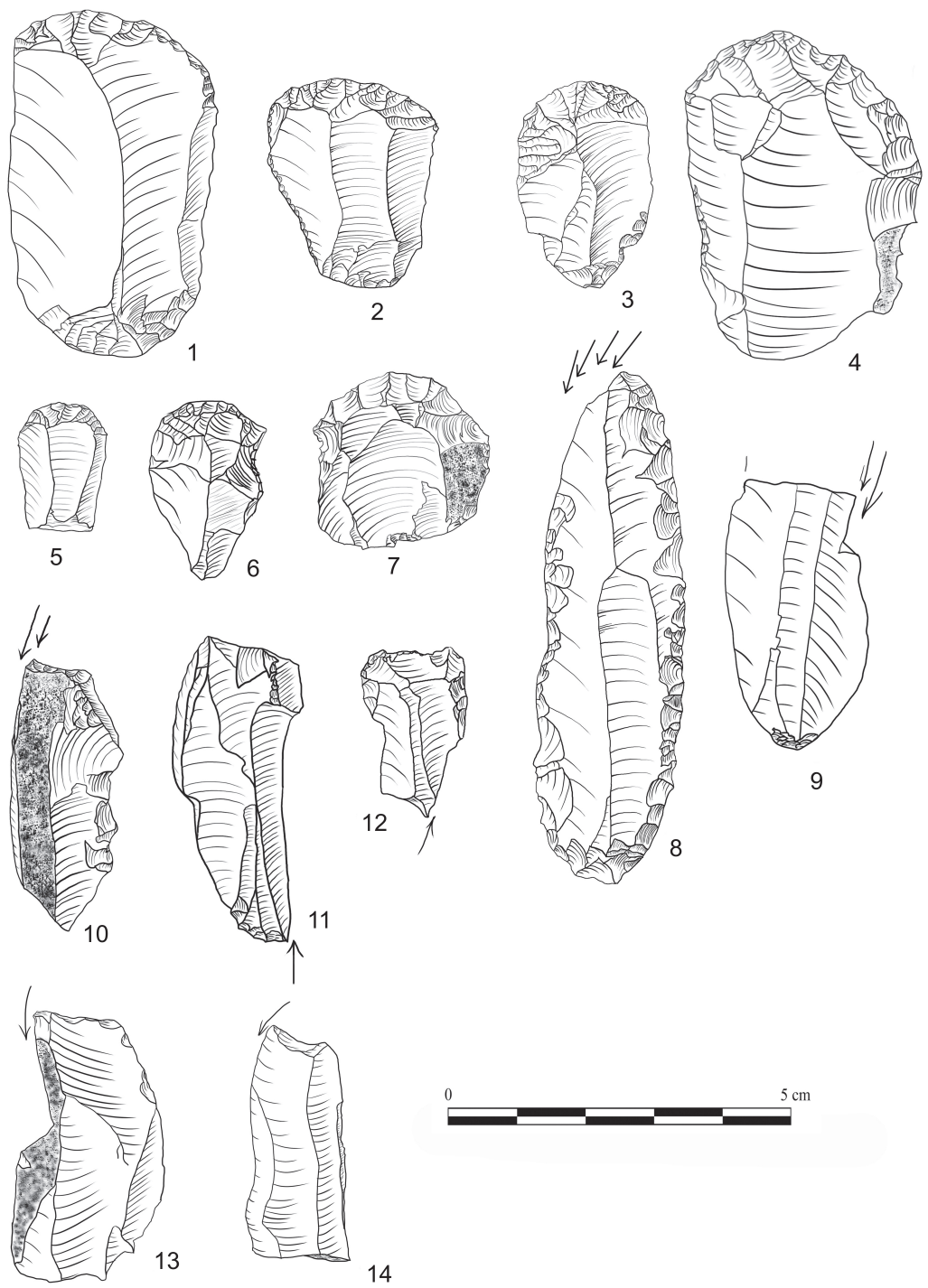

Fig. 3. Domestic tools of the studied sites. 1 - Arka; 2, 3, 8 - Bodrogkeresztúr; 4, 9 - Hidasnémeti; 5, 7, 10, 11 - Ságvár; 6, 12 - Corvin-tér; 13 - Esztergom; 14 - Nadap; 1-7 - end-scrapers; 8-14 - burins 
points with slightly convex back were curved back points. Arched backed points have a smaller radius of back curvature near the tip therefore the tip is rather in an offset position. Vachons point, shouldered point, fléchette, and bifacial leaf points were further point types (Demars, LaURent 1992).

The rest of the tools, end-scrapers, burins, edge retouched tools, borer, truncated tools, splintered tool, notched-denticulated and combined tool, were regarded here as domestic tools (Fig. 3). These classes were not further divided into subtypes. This simplification was made to reduce the number of typological classes of the domestic tools that are often impractical to differentiate cultures in contrast to the armature types.

\section{ARKA}

\section{The site}

The site is located in northeast Hungary in the western zone of Tokaj Mountains, at village Arka. The site was found $255 \mathrm{~m}$ a.s.l. near stream Arka on the western slope of Magoska hill $734 \mathrm{~m}$ a.s.l. The main river of this area is the Hernád that flows $4 \mathrm{~km}$ westwards but its view from the site is blocked by a ridge rising up to $280 \mathrm{~m}$ a.s.l.

The site was excavated in 1960, 1961, and 1963 by L. VéRTES $(1962,1964-1965)$. The excavation was carried out on a slope where two narrow 2-3 $\mathrm{m}$ deep converging gullies cut the surface. Between the gullies the widest area was $c a .12 \mathrm{~m}$. The main part of the excavation was carried out in this $12 \mathrm{~m}$ wide strip of land.

The stratigraphy consisted of the recent soil cover, than a humic soil of Holocene age, under which the Pleistocene sequence begun. The Pleistocene layers were severely reworked by cryoturbation down to the andesite bedrock. The surface of the Pleistocene layers showed also polygonal forms. The interface with the bedrock was uneven.

The lithic material appeared in varying elevations and the finds were scattered between 30 and $80 \mathrm{~cm}$ thickness of the sediment. VÉRTES claimed the lithics had been recovered from two levels separated by nearly $1 \mathrm{~m}$ sterile sediment. The thickness of the lower layer was $c a .30 \mathrm{~cm}$. The lower archaeological level preserved a hearth with an andesite structure 2 meters under the actual surface. This feature in the original field notes of VÉRTES was described as a cluster of charcoals, bones and lithics, and an angular flat surfaced andesite rock. Another rock with a depression in the middle bore ochre stain. The find distribution, however, showed that where material appeared in the lower level, in the very same square, the upper level was empty or scarce of lithics. Lithic refitting tested the displacement of artifacts throughout the two layers and found matching pieces. This result supports VÉRTES' observation that cryoturbation admixed the sediments and contradicts the existence of two occupational layers at the site.

Besides the hearths, further charcoals were found in pockets and scattered animal bones and teeth of poor preservation.

VÉRTES (1964-1965) defined the assemblage as Eastern Gravettian. Later the site became a representative of the Epigravettian (Dовоsi 2009a). Because the material 
consists of a large quantity of locally available raw material, it was defined as a workshop site. VÉRTES claimed that near the site there are rock blocks enclosing limnic silicite, which can be confirmed by a recent field survey.

The first ${ }^{14} \mathrm{C}$ date for Arka, $17050 \pm 350$ (GrN-4038), was obtained on charcoals of the 1961 excavation (Vogel, Waterbolk 1964). Its sample derived from the hearth of the lower layer. During the sample cleaning all organic matter dissolved in alkali and this fraction was measured. According to the laboratory, the true age may thus be higher (Vogel, Waterbolk 1964). The second date is $13230 \pm 85$ (GrN-4218). The sample is hearth filling from $2 \mathrm{~m}$ depth in trench M excavated in 1963 (VOGEL, WaterbolK 1967). In the field notes of VÉRTES, the location of the sample was a small but thick charcoal layer next to which lithic raw material blocks were found. The report on this radiocarbon date by VOGEL and WATERBOLK (1967) mentions that VérTES submitted the sample as taken from the lower layer but later changed the stratigraphic identity to the upper layer. Henceforth, this date has been associated with the upper archaeological level (GÁBori-CsánK 1970; Dobosi, SzÁntó 2003). The third date, $18600 \pm 1900$ (A-518) (HAYNES et al. 1966), was obtained from charcoals of the 1963 excavation season. The sample was taken from within a small patch of charcoal $75 \mathrm{~cm}$ beneath the former sample of the same excavation block. The sampled level was situated in the lower third part of an archaeologically sterile portion of the site stratigraphy. The next level of finds was located $25 \mathrm{~cm}$ beneath this sampled charcoal patch. On the sample submission form the lower archaeological layer is indicated as the sample's relative chronological position. This date appears inaccurately $18700 \pm 190$ in summaries of Hungarian ${ }^{14} \mathrm{C}$ dates (GÁBORI-CsÁNK 1970; DoBosi, SzÁnTó 2003). The admixture at Arka also might have modified the original position of the organic remains sampled for dating. It is, therefore, difficult to make authentic association between radiocarbon samples and the artifacts. The effect of cryoturbation upon the sediments of the site raises the insecurity of the stratigraphic position of the $13 \mathrm{ka}$ BP date whose sample was originally submitted from the lower layer but after the young dating result compared to the $17 \mathrm{k}$ years date VÉRTES changed the archaeological stratigraphic position of the sample with the upper layer. The Epigravettian age for the site thus was found unsupportable (LENGYEL 2008-2009), and the typological character of the assemblage suited the Late Gravettian inventory of Bodrogkeresztúr (LENGYEL 2016).

The lithic sample used in this study derived from adjacent trenches A, B, C, D, M, N, $\mathrm{P}$ and $\mathrm{L}$ of the 1961 and 1963 excavations. The material does not include the total number of recovered find, because VÉRTES did not collect all flakes, chunks, and debris of the local raw material, but the blades, cores, tools, and each piece of non-local raw materials. In spite of this, the assemblage may represent a sample from the occupation.

\section{Raw materials}

Local materials make up $81.5 \%$ of the total assemblage (Tab. 1) which are the limnic silicite outcropping in large quantity in the local stream bed within 100 meters distance west and north of the site. These weigh $101 \mathrm{~kg}$ while all the other materials make up a few kilograms of the assemblage. The local raw materials yielded 62.4 item per $\mathrm{kg}$. 
Regional raw materials make up $13.6 \%$ of the total assemblage. This group consists of the eastern Tokaj materials such as the $\mathrm{C} 1$ and $\mathrm{C} 2$ obsidian, red jasper, the eastern Slovakian radiolarite, black quartzite and menilite, and the meta-rhyolite of the Bükk Mountains. Latter is present only with a single item. Regional materials yielded 143.7 item per $\mathrm{kg}$.

Transcarpathian (TC) materials make up $4.9 \%$ of the total assemblage. These are the Jurassic flint from the Kraków-Częstochowa upland, the erratic flint from Silesia/ Moravian Gate, and the Volyn-Podolian flints $270 \mathrm{~km}$ northeast. TC materials yielded 197.3 item per $\mathrm{kg}$.

\section{Blade tool production}

The local and regional raw materials have all the technological categories while the transcarpathian group lacks flake cores (Tab. 2). According to the dominancy of blade cores and the high ratio of blades in the tool assemblage $(70.9 \%)$, the dominant knapping activity was the blade production. In spite of the mass of flakes, flake cores make up only $3.1 \%$ of the total core assemblage.

Overall, unidirectional removal scars dominate the blades (Tab. 3) and there is a predominance of single striking platform blade cores (Tab. 4). Besides, there is an important percent of perpendicular scars, which is due to the abundancy of neo-crested blades (Tab. 2). The presence of blade cores of two striking platforms shows bidirectional core reduction, as well. The blade platforms are predominantly plain (Tab. 5) and there is an insignificant ratio of platform faceting. The absence of impact point and the frequent overhang abrasion signal soft hammer technique in the blade debitage (Tab. 6).

Different means of blade sizes were measured by raw material groups (Tab. 7). The ANOVA test (Tab. 8) showed that local blades are significantly longer, wider, and thicker than regional and TC ones, while regional and TC items are statistically similar. Also, the measurements of the unbroken blade cores (Tab. 9) showed that local specimens are greater in each dimension (Tab. 10).

Blades highly dominate the tool assemblage (Tab. 11) with $9.3 \%$ of all blades being tools, while the tool frequency among flakes is $2.6 \%$ (Tab. 12). Breaking down the ratio by raw materials, observed is that the larger the distance between site and lithic source, the greater the ratio of blades used up for making tool.

The blade tools (Tab. 13) are generally shorter than the blanks while there are no differences concerning width and thickness (Tab. 14). The difference in length is due to that $84 \%$ of the blade tools are broken or severely shortened by retouching.

Comparing the sizes of the blade tools by raw materials (Tab. 15), ANOVA found that blade tools of local raw material are longer, wider and thicker than the blade tools of the other two raw materials. This coincides with the observation on the blank blade mean values of length, width and thickness.

Most blade tools are domestic types, but the armature group is significant (Tab. 16). Most local blade tools are end-scrapers, while regional and TC blade tools tend to be armatures. 
The armatures are mostly backed bladelets (Tab. 17), and backed truncated types also are numerous, including a trapeze-rectangle and a backed ventrally truncated type. Points are tip retouched blades and microgravette types besides a single specimen of fléchette (Tab. 18).

\section{Flake tool production}

The very few remains of flake debitage are six flake cores. This allowed to deduce that the numerous flakes of the local material indeed are by-products of the blade debitage. Out of the six flake cores four were made of local raw materials and two are of regional.

Unidirectional scars are the minority on the local and regional flakes, but TC flakes were removed with unidirectional knapping orientation (Tab. 19). The flake platforms are plain in most cases (Tab. 20) and have impact points and unabraded overhangs, showing the extensive use of hard hammer technique (Tab. 21).

The largest flakes were found among the local materials (Tab. 22). When the mean lengths, widths and thicknesses of the flakes are compared by raw materials, ANOVA showed local flakes larger (Tab. 23).

The flakes compose the minority of the toolkit (Tab. 11). Out of all flakes only $2.6 \%$ were used for tools, which is a lower portion than what was found concerning the blades (Tab. 12). Most flake tools were made of the local raw materials (Tab. 11), but a greater portion of the regional and TC flakes, respectively, were used for making tools (Tab. 12).

Local flake tools are larger than the others (Tab. 24). There are differences between blank and tool flakes sizes, too (Tab. 25). The t-test comparison showed that local raw material flake tools are narrower than the blank items. Concerning regional and TC raw materials, flake tools are considerably thicker than the blank ones.

Comparing the sizes of the flake tools by raw materials (Tab. 26), ANOVA found local flake tools wider than the others.

End-scrapers and burins are the most common flake tools (Tab. 27). Local materials were used to make all the types made of flakes, while regional material did not yield borer and composite tool. TC items are end-scraper, burin and an edge retouched tool. The notched-denticulated tool production seems to be in sole association with the local materials. There are also two end-scrapers made of core platform rejuvenating flakes of local raw materials. 
Lithic analysis of the Middle and Late Upper Palaeolithic in Hungary

Table 1. Arka lithic raw material composition by weight in grams

\begin{tabular}{|l|c|c|c|c|c|c|}
\hline Raw material & Blade & Flake & Core & Raw Block & Total & Total \% \\
\hline Local & 10863 & 64595 & 20698 & 4986 & 101142 & 88.76076 \\
\hline Within local \% & 10.74035 & 63.86565 & 20.4643 & 4.929703 & 100 & \\
\hline Regional & 1124 & 3620 & 2231 & 3997 & 10972 & 9.628869 \\
\hline Within regional \% & 10.24426 & 32.99307 & 20.33358 & 36.42909 & 100 & \\
\hline Transcarpathian & 724 & 696 & 415 & & 1835 & 1.61037 \\
\hline Within transcarpathian \% & 39.45504 & 37.92916 & 22.6158 & 0 & 100 & \\
\hline Total & 12711 & 68911 & 23344 & 8983 & 113949 & 100 \\
\hline Within total \% & 11.15499 & 60.4753 & 20.48636 & 7.883351 & 100 & \\
\hline
\end{tabular}

Table 2. Arka lithic assemblage composition by raw material types and technological categories

\begin{tabular}{|c|c|c|c|c|c|c|}
\hline & \multicolumn{3}{|c|}{ Raw material } & \multirow{2}{*}{ Total } \\
\hline & & & local & regional & transcarpathian & \\
\hline \multirow[t]{16}{*}{ Class } & \multirow[t]{2}{*}{ flake } & count & 2086 & 277 & 95 & 2458 \\
\hline & & $\%$ within raw material & $34.7 \%$ & $27.6 \%$ & $26.2 \%$ & $33.4 \%$ \\
\hline & \multirow[t]{2}{*}{ blade } & count & 1032 & 410 & 182 & 1624 \\
\hline & & $\%$ within raw material & $17.2 \%$ & $40.9 \%$ & $50.3 \%$ & $22.0 \%$ \\
\hline & \multirow[t]{2}{*}{ debris } & count & 2612 & 207 & 59 & 2878 \\
\hline & & $\%$ within raw material & $43.5 \%$ & $20.7 \%$ & $16.3 \%$ & $39.1 \%$ \\
\hline & \multirow{2}{*}{$\begin{array}{l}\text { rejuvenating } \\
\text { flake }\end{array}$} & count & 65 & 12 & 4 & 81 \\
\hline & & $\%$ within raw material & $1.1 \%$ & $1.2 \%$ & $1.1 \%$ & $1.1 \%$ \\
\hline & \multirow[t]{2}{*}{ crest } & count & 14 & 2 & 1 & 17 \\
\hline & & $\%$ within raw material & $0.2 \%$ & $0.2 \%$ & $0.3 \%$ & $0.2 \%$ \\
\hline & \multirow[t]{2}{*}{ neo-crest } & count & 74 & 28 & 11 & 113 \\
\hline & & $\%$ within raw material & $1.2 \%$ & $2.8 \%$ & $3.0 \%$ & $1.5 \%$ \\
\hline & \multirow[t]{2}{*}{ blade core } & count & 116 & 64 & 10 & 190 \\
\hline & & $\%$ within raw material & $1.9 \%$ & $6.4 \%$ & $2.8 \%$ & $2.6 \%$ \\
\hline & \multirow[t]{2}{*}{ flake core } & count & 4 & 2 & 0 & 6 \\
\hline & & $\%$ within raw material & $0.1 \%$ & $0.2 \%$ & $0.0 \%$ & $0.1 \%$ \\
\hline \multirow{2}{*}{\multicolumn{2}{|c|}{ Total }} & count & 6003 & 1002 & 362 & 7367 \\
\hline & & $\%$ within raw material & $100.0 \%$ & $100.0 \%$ & $100.0 \%$ & $100.0 \%$ \\
\hline
\end{tabular}

Table 3. Arka blade assemblage (complete specimens) dorsal scar pattern frequency by raw material

\begin{tabular}{|c|c|c|c|c|c|c|}
\hline & \multicolumn{3}{|c|}{ Raw material } & \multirow{2}{*}{ Total } \\
\hline & & & local & regional & transcarpathian & \\
\hline \multirow[t]{10}{*}{ Scars } & \multirow[t]{2}{*}{ unidirectional } & count & 144 & 54 & 40 & 238 \\
\hline & & $\%$ within raw material & $49.0 \%$ & $59.3 \%$ & $53.3 \%$ & $51.7 \%$ \\
\hline & \multirow[t]{2}{*}{ opposite } & count & 58 & 18 & 17 & 93 \\
\hline & & $\%$ within raw material & $19.7 \%$ & $19.8 \%$ & $22.7 \%$ & $20.2 \%$ \\
\hline & \multirow[t]{2}{*}{ perpendicular } & count & 73 & 14 & 14 & 101 \\
\hline & & $\%$ within raw material & $24.8 \%$ & $15.4 \%$ & $18.7 \%$ & $22.0 \%$ \\
\hline & \multirow[t]{2}{*}{ multiple } & count & 18 & 4 & 4 & 26 \\
\hline & & $\%$ within raw material & $6.1 \%$ & $4.4 \%$ & $5.3 \%$ & $5.7 \%$ \\
\hline & \multirow[t]{2}{*}{ no scar } & count & 1 & 1 & 0 & 2 \\
\hline & & $\%$ within raw material & $0.3 \%$ & $1.1 \%$ & $0.0 \%$ & $0.4 \%$ \\
\hline \multirow{2}{*}{\multicolumn{2}{|c|}{ Total }} & count & 294 & 91 & 75 & 460 \\
\hline & & $\%$ within raw material & $100.0 \%$ & $100.0 \%$ & $100.0 \%$ & $100.0 \%$ \\
\hline
\end{tabular}


Table 4. Arka blade core types by raw material

\begin{tabular}{|c|c|c|c|c|c|c|}
\hline & \multicolumn{3}{|c|}{ Raw material } & \multirow{2}{*}{ Total } \\
\hline & & & local & regional & transcarpathian & \\
\hline \multirow[t]{12}{*}{ Types } & \multirow[t]{2}{*}{ unidirectional } & count & 65 & 21 & 6 & 92 \\
\hline & & $\%$ within raw material & $56.0 \%$ & $32.8 \%$ & $60.0 \%$ & $48.4 \%$ \\
\hline & \multirow[t]{2}{*}{ bidirectional } & count & 7 & 2 & 0 & 9 \\
\hline & & $\%$ within raw material & $6.0 \%$ & $3.1 \%$ & $0.0 \%$ & $4.7 \%$ \\
\hline & \multirow[t]{2}{*}{ alternate } & count & 19 & 12 & 4 & 35 \\
\hline & & $\%$ within raw material & $16.4 \%$ & $18.8 \%$ & $40.0 \%$ & $18.4 \%$ \\
\hline & \multirow[t]{2}{*}{ multidirectional } & count & 2 & 0 & 0 & 2 \\
\hline & & $\%$ within raw material & $1.7 \%$ & $0.0 \%$ & $0.0 \%$ & $1.1 \%$ \\
\hline & \multirow[t]{2}{*}{ pre-core } & count & 2 & 2 & 0 & 4 \\
\hline & & $\%$ within raw material & $1.7 \%$ & $3.1 \%$ & $0.0 \%$ & $2.1 \%$ \\
\hline & \multirow[t]{2}{*}{ core fragment } & count & 21 & 27 & 0 & 48 \\
\hline & & $\%$ within raw material & $18.1 \%$ & $42.2 \%$ & $0.0 \%$ & $25.3 \%$ \\
\hline \multirow{2}{*}{\multicolumn{2}{|c|}{ Total }} & count & 116 & 64 & 10 & 190 \\
\hline & & $\%$ within raw material & $100.0 \%$ & $100.0 \%$ & $100.0 \%$ & $100.0 \%$ \\
\hline
\end{tabular}

Table 5. Arka blade assemblage (complete specimens) platform type frequency by raw material

\begin{tabular}{|c|c|c|c|c|c|c|}
\hline & \multicolumn{3}{|c|}{ Raw material } & \multirow{2}{*}{ Total } \\
\hline & & & local & regional & transcarpathian & \\
\hline \multirow[t]{12}{*}{ Platform } & \multirow[t]{2}{*}{ plain } & count & 241 & 68 & 54 & 363 \\
\hline & & $\%$ within raw material & $82.0 \%$ & $74.7 \%$ & $72.0 \%$ & $78.9 \%$ \\
\hline & \multirow[t]{2}{*}{ dihedral } & count & 19 & 9 & 1 & 29 \\
\hline & & $\%$ within raw material & $6.5 \%$ & $9.9 \%$ & $1.3 \%$ & $6.3 \%$ \\
\hline & \multirow[t]{2}{*}{ faceted } & count & 16 & 5 & 3 & 24 \\
\hline & & $\%$ within raw material & $5.4 \%$ & $5.5 \%$ & $4.0 \%$ & $5.2 \%$ \\
\hline & \multirow[t]{2}{*}{ cortical } & count & 4 & 3 & 3 & 10 \\
\hline & & $\%$ within raw material & $1.4 \%$ & $3.3 \%$ & $4.0 \%$ & $2.2 \%$ \\
\hline & \multirow[t]{2}{*}{ linear } & count & 12 & 6 & 14 & 32 \\
\hline & & $\%$ within raw material & $4.1 \%$ & $6.6 \%$ & $18.7 \%$ & $7.0 \%$ \\
\hline & \multirow[t]{2}{*}{ punctiform } & count & 2 & 0 & 0 & 2 \\
\hline & & $\%$ within raw material & $0.7 \%$ & $0.0 \%$ & $0.0 \%$ & $0.4 \%$ \\
\hline \multirow{2}{*}{\multicolumn{2}{|c|}{ Total }} & count & 294 & 91 & 75 & 460 \\
\hline & & $\%$ within raw material & $100.0 \%$ & $100.0 \%$ & $100.0 \%$ & $100.0 \%$ \\
\hline
\end{tabular}


Table 6. Arka blade assemblage (complete specimens) impact point-overhang co-presence by raw material

\begin{tabular}{|c|c|c|c|c|c|c|}
\hline & \multicolumn{3}{|c|}{ Raw material } & \multirow{2}{*}{ Total } \\
\hline & & & local & regional & transcarpathian & \\
\hline \multirow{8}{*}{$\begin{array}{l}\text { Impact point } \\
\text {-overhang }\end{array}$} & \multirow[t]{2}{*}{ none } & count & 166 & 70 & 64 & 300 \\
\hline & & $\%$ within raw material & $56.5 \%$ & $76.9 \%$ & $85.3 \%$ & $65.2 \%$ \\
\hline & \multirow[t]{2}{*}{ yes-no } & count & 30 & 1 & 6 & 37 \\
\hline & & $\%$ within raw material & $10.2 \%$ & $1.1 \%$ & $8.0 \%$ & $8.0 \%$ \\
\hline & \multirow[t]{2}{*}{ yes-yes } & count & 42 & 8 & 0 & 50 \\
\hline & & $\%$ within raw material & $14.3 \%$ & $8.8 \%$ & $0.0 \%$ & $10.9 \%$ \\
\hline & \multirow[t]{2}{*}{ no-yes } & count & 56 & 12 & 5 & 73 \\
\hline & & $\%$ within raw material & $19.0 \%$ & $13.2 \%$ & $6.7 \%$ & $15.9 \%$ \\
\hline \multirow{2}{*}{\multicolumn{2}{|c|}{ Total }} & count & 294 & 91 & 75 & 460 \\
\hline & & $\%$ within raw material & $100.0 \%$ & $100.0 \%$ & $100.0 \%$ & $100.0 \%$ \\
\hline
\end{tabular}

Table 7. Arka blade assemblage (complete specimens) length, width and thickness by raw material

\begin{tabular}{|c|c|c|c|c|}
\hline \multicolumn{2}{|l|}{ Raw material } & Length [mm] & Width [mm] & Thickness [mm] \\
\hline \multirow[t]{6}{*}{ Local } & Minimum & 7.50 & 6.20 & 1.50 \\
\hline & Maximum & 133.20 & 93.80 & 26.90 \\
\hline & Mean & 56.5611 & 21.7852 & 8.4279 \\
\hline & Median & 52.2000 & 21.0000 & 7.3000 \\
\hline & $\mathrm{N}$ & 294 & 294 & 294 \\
\hline & Std. deviation & 21.83817 & 9.39898 & 4.82171 \\
\hline \multirow[t]{6}{*}{ Regional } & Minimum & 4.10 & 6.70 & 2.20 \\
\hline & Maximum & 115.60 & 28.80 & 15.10 \\
\hline & Mean & 39.0144 & 14.1158 & 5.4319 \\
\hline & Median & 38.4000 & 13.0000 & 4.6000 \\
\hline & $\mathrm{N}$ & 91 & 91 & 91 \\
\hline & Std. deviation & 14.68085 & 5.19578 & 2.67916 \\
\hline \multirow[t]{6}{*}{ Transcarpathian } & Minimum & 20.30 & 6.20 & 1.90 \\
\hline & Maximum & 80.00 & 43.10 & 12.80 \\
\hline & Mean & 42.5560 & 14.7080 & 4.6645 \\
\hline & Median & 41.4000 & 13.4000 & 3.9000 \\
\hline & $\mathrm{N}$ & 75 & 75 & 75 \\
\hline & Std. deviation & 13.84572 & 5.80272 & 2.26361 \\
\hline \multirow[t]{6}{*}{ Total } & Minimum & 4.10 & 6.20 & 1.50 \\
\hline & Maximum & 133.20 & 93.80 & 26.90 \\
\hline & Mean & 50.8065 & 19.1141 & 7.2216 \\
\hline & Median & 46.2500 & 18.0500 & 6.0000 \\
\hline & $\mathrm{N}$ & 460 & 460 & 460 \\
\hline & Std. deviation & 20.91648 & 8.93358 & 4.43958 \\
\hline
\end{tabular}


Table 8. Arka blade assemblage (complete specimens) ANOVA and Tukey post hoc test to compare length, width and thickness by raw materials

ANOVA

\begin{tabular}{|l|l|r|r|r|r|c|}
\hline \multicolumn{2}{|c|}{} & Sum of squares & df & Mean square & F & Sig. \\
\hline \multirow{3}{*}{ Length $[\mathrm{mm}]$} & between groups & 27495.090 & 2 & 13747.545 & 36.249 & 0.000 \\
\cline { 2 - 7 } & within groups & 173316.936 & 457 & 379.249 & & \\
\cline { 2 - 7 } & Total & 200812.027 & 459 & & & \\
\hline \multirow{3}{*}{ Width $[\mathrm{mm}]$} & between groups & 5827.037 & 2 & 2913.518 & 43.223 & 0.000 \\
\cline { 2 - 7 } & within groups & 30805.203 & 457 & 67.407 & & \\
\cline { 2 - 7 } & Total & 36632.240 & 459 & & & \\
\hline \multirow{2}{*}{ Thickness $[\mathrm{mm}]$} & between groups & 1209.715 & 2 & 604.857 & 35.271 & 0.000 \\
\cline { 2 - 7 } & within groups & 7837.100 & 457 & 17.149 & & \\
\cline { 2 - 7 } & Total & 9046.814 & 459 & & & \\
\hline
\end{tabular}

Multiple Comparisons

Tukey HSD

\begin{tabular}{|c|c|c|c|c|c|c|c|}
\hline \multirow{2}{*}{$\begin{array}{l}\text { Dependent } \\
\text { variable }\end{array}$} & \multirow{2}{*}{ (I) raw material } & \multirow{2}{*}{ (J) raw material } & \multirow{2}{*}{$\begin{array}{c}\text { Mean } \\
\text { difference } \\
(\mathrm{I}-\mathrm{J})\end{array}$} & \multirow{2}{*}{$\begin{array}{l}\text { Std. } \\
\text { error }\end{array}$} & \multirow{2}{*}{ Sig. } & \multicolumn{2}{|c|}{$\begin{array}{l}\text { 95\% Confidence } \\
\text { interval }\end{array}$} \\
\hline & & & & & & $\begin{array}{l}\text { Lower } \\
\text { bound }\end{array}$ & $\begin{array}{l}\text { Upper } \\
\text { bound }\end{array}$ \\
\hline \multirow{6}{*}{$\begin{array}{l}\text { Length } \\
{[\mathrm{mm}]}\end{array}$} & \multirow[t]{2}{*}{ local } & regional & 17.54669 & 2.33614 & 0.000 & 12.0536 & 23.0398 \\
\hline & & transcarpathian & 14.00509 & 2.51925 & 0.000 & 8.0814 & 19.9288 \\
\hline & \multirow[t]{2}{*}{ regional } & local & -17.54669 & 2.33614 & 0.000 & -23.0398 & -12.0536 \\
\hline & & transcarpathian & -3.54160 & 3.03714 & 0.474 & -10.6831 & 3.5999 \\
\hline & \multirow[t]{2}{*}{ transcarpathian } & local & -14.00509 & 2.51925 & 0.000 & -19.9288 & -8.0814 \\
\hline & & regional & 3.54160 & 3.03714 & 0.474 & -3.5999 & 10.6831 \\
\hline \multirow{6}{*}{$\begin{array}{l}\text { Width } \\
{[\mathrm{mm}]}\end{array}$} & \multirow[t]{2}{*}{ local } & regional & 7.66935 & 0.98490 & 0.000 & 5.3535 & 9.9852 \\
\hline & & transcarpathian & 7.07717 & 1.06209 & 0.000 & 4.5798 & 9.5745 \\
\hline & \multirow[t]{2}{*}{ regional } & local & -7.66935 & 0.98490 & 0.000 & -9.9852 & -5.3535 \\
\hline & & transcarpathian & -0.59218 & 1.28043 & 0.889 & -3.6030 & 2.4186 \\
\hline & \multirow[t]{2}{*}{ transcarpathian } & local & -7.07717 & 1.06209 & 0.000 & -9.5745 & -4.5798 \\
\hline & & regional & 0.59218 & 1.28043 & 0.889 & -2.4186 & 3.6030 \\
\hline \multirow{6}{*}{$\begin{array}{l}\text { Thickness } \\
{[\mathrm{mm}]}\end{array}$} & \multirow[t]{2}{*}{ local } & regional & 2.99606 & 0.49677 & 0.000 & 1.8280 & 4.1641 \\
\hline & & transcarpathian & 3.76339 & 0.53571 & 0.000 & 2.5037 & 5.0230 \\
\hline & \multirow[t]{2}{*}{ regional } & local & -2.99606 & 0.49677 & 0.000 & -4.1641 & -1.8280 \\
\hline & & transcarpathian & 0.76733 & 0.64584 & 0.461 & -0.7513 & 2.2859 \\
\hline & \multirow[t]{2}{*}{\begin{tabular}{|l|} 
transcarpathian \\
\end{tabular}} & local & -3.76339 & 0.53571 & 0.000 & -5.0230 & -2.5037 \\
\hline & & regional & -0.76733 & 0.64584 & 0.461 & -2.2859 & 0.7513 \\
\hline
\end{tabular}


Lithic analysis of the Middle and Late Upper Palaeolithic in Hungary

Table 9. Arka blade core (complete specimens) size by raw material

\begin{tabular}{|c|c|c|c|c|}
\hline \multicolumn{2}{|l|}{ Raw material } & Length & Width & Depth \\
\hline \multirow[t]{6}{*}{ Local } & Minimum & 24.20 & 16.60 & 11.10 \\
\hline & Maximum & 148.00 & 96.30 & 90.00 \\
\hline & Mean & 65.9774 & 40.7645 & 43.8226 \\
\hline & Median & 61.3000 & 39.9000 & 39.4000 \\
\hline & $\mathrm{N}$ & 93 & 93 & 93 \\
\hline & Std. deviation & 22.07892 & 15.07795 & 17.18868 \\
\hline \multirow[t]{6}{*}{ Regional } & Minimum & 25.00 & 12.40 & 11.50 \\
\hline & Maximum & 60.70 & 40.30 & 41.40 \\
\hline & Mean & 35.9057 & 24.8514 & 27.2543 \\
\hline & Median & 34.6000 & 24.2000 & 27.2000 \\
\hline & $\mathrm{N}$ & 35 & 35 & 35 \\
\hline & Std. deviation & 8.37777 & 7.62775 & 7.71078 \\
\hline \multirow[t]{6}{*}{ Transcarpathian } & Minimum & 23.90 & 13.80 & 11.00 \\
\hline & Maximum & 62.60 & 39.70 & 56.70 \\
\hline & Mean & 43.6200 & 25.1600 & 29.9900 \\
\hline & Median & 40.7000 & 24.1000 & 28.7000 \\
\hline & $\mathrm{N}$ & 10 & 10 & 10 \\
\hline & Std. deviation & 11.49355 & 8.58075 & 12.43958 \\
\hline \multirow[t]{6}{*}{ Total } & Minimum & 23.90 & 12.40 & 11.00 \\
\hline & Maximum & 148.00 & 96.30 & 90.00 \\
\hline & Mean & 56.7304 & 35.5978 & 38.6181 \\
\hline & Median & 53.5500 & 33.6500 & 34.2500 \\
\hline & $\mathrm{N}$ & 138 & 138 & 138 \\
\hline & Std. deviation & 23.12654 & 15.08386 & 16.73733 \\
\hline
\end{tabular}

Table 10. Arka blade core assemblage (complete specimens) ANOVA and Tukey post hoc test to compare length, width and thickness by raw materials

ANOVA

\begin{tabular}{|l|l|r|r|r|r|c|}
\hline \multicolumn{2}{|c|}{} & Sum of squares & df & Mean square & F & Sig. \\
\hline \multirow{3}{*}{ Length } & between groups & 24849.375 & 2 & 12424.687 & 34.639 & 0.000 \\
\cline { 2 - 7 } & within groups & 48423.297 & 135 & 358.691 & & \\
\cline { 2 - 7 } & Total & 73272.672 & 137 & & & \\
\hline \multirow{4}{*}{ Width } & between groups & 7614.065 & 2 & 3807.033 & 21.818 & 0.000 \\
\cline { 2 - 7 } & within groups & 23556.564 & 135 & 174.493 & & \\
\cline { 2 - 7 } & Total & 31170.629 & 137 & & & \\
\hline \multirow{3}{*}{ Depth } & between groups & 7783.266 & 2 & 3891.633 & 17.171 & 0.000 \\
\cline { 2 - 7 } & within groups & 30595.658 & 135 & 226.635 & & \\
\cline { 2 - 7 } & Total & 38378.925 & 137 & & & \\
\hline
\end{tabular}


Table 10. Continued

Multiple Comparisons

Tukey HSD

\begin{tabular}{|c|c|c|c|c|c|c|c|}
\hline \multirow{2}{*}{$\begin{array}{l}\text { Dependent } \\
\text { variable }\end{array}$} & \multirow{2}{*}{ (I) raw material } & \multirow{2}{*}{ (J) raw material } & \multirow{2}{*}{$\begin{array}{c}\text { Mean } \\
\text { difference } \\
(\mathrm{I}-\mathrm{J})\end{array}$} & \multirow{2}{*}{$\begin{array}{l}\text { Std. } \\
\text { error }\end{array}$} & \multirow{2}{*}{ Sig. } & \multicolumn{2}{|c|}{$\begin{array}{l}\text { 95\% Confidence } \\
\text { interval }\end{array}$} \\
\hline & & & & & & $\begin{array}{l}\text { Lower } \\
\text { bound }\end{array}$ & $\begin{array}{l}\text { Upper } \\
\text { bound }\end{array}$ \\
\hline \multirow[t]{6}{*}{ Length } & \multirow[t]{2}{*}{ local } & regional & 30.07171 & 3.75569 & 0.000 & 21.1713 & 38.9721 \\
\hline & & transcarpathian & 22.35742 & 6.30286 & 0.002 & 7.4206 & 37.2942 \\
\hline & \multirow[t]{2}{*}{ regional } & local & -30.07171 & 3.75569 & 0.000 & -38.9721 & -21.1713 \\
\hline & & transcarpathian & -7.71429 & 6.79098 & 0.494 & -23.8079 & 8.3793 \\
\hline & \multirow[t]{2}{*}{ transcarpathian } & local & -22.35742 & 6.30286 & 0.002 & -37.2942 & -7.4206 \\
\hline & & regional & 7.71429 & 6.79098 & 0.494 & -8.3793 & 23.8079 \\
\hline \multirow[t]{6}{*}{ Width } & \multirow[t]{2}{*}{ local } & regional & 15.91309 & 2.61950 & 0.000 & 9.7053 & 22.1209 \\
\hline & & transcarpathian & 15.60452 & 4.39609 & 0.002 & 5.1865 & 26.0226 \\
\hline & \multirow[t]{2}{*}{ regional } & local & -15.91309 & 2.61950 & 0.000 & -22.1209 & -9.7053 \\
\hline & & transcarpathian & -0.30857 & 4.73654 & 0.998 & -11.5334 & 10.9163 \\
\hline & \multirow[t]{2}{*}{ transcarpathian } & local & -15.60452 & 4.39609 & 0.002 & -26.0226 & -5.1865 \\
\hline & & regional & 0.30857 & 4.73654 & 0.998 & -10.9163 & 11.5334 \\
\hline \multirow[t]{6}{*}{ Depth } & \multirow[t]{2}{*}{ local } & regional & 16.56829 & 2.98533 & 0.000 & 9.4935 & 23.6431 \\
\hline & & transcarpathian & 13.83258 & 5.01003 & 0.018 & 1.9596 & 25.7056 \\
\hline & \multirow[t]{2}{*}{ regional } & local & -16.56829 & 2.98533 & 0.000 & -23.6431 & -9.4935 \\
\hline & & transcarpathian & -2.73571 & 5.39803 & 0.868 & -15.5282 & 10.0568 \\
\hline & \multirow[t]{2}{*}{ transcarpathian } & local & -13.83258 & 5.01003 & 0.018 & -25.7056 & -1.9596 \\
\hline & & regional & 2.73571 & 5.39803 & 0.868 & -10.0568 & 15.5282 \\
\hline
\end{tabular}

Table 11. Arka tool assemblage product composition by raw materials

\begin{tabular}{|c|c|c|c|c|c|}
\hline & \multicolumn{3}{|c|}{ Raw material } & \multirow{2}{*}{ Total } \\
\hline & & local & regional & transcarpathian & \\
\hline \multirow[t]{2}{*}{ Flake } & count & 35 & 22 & 8 & 65 \\
\hline & $\%$ within raw material & $36.5 \%$ & $24.4 \%$ & $18.2 \%$ & $28.3 \%$ \\
\hline \multirow[t]{2}{*}{ Blade } & count & 59 & 68 & 36 & 163 \\
\hline & $\%$ within raw material & $61.5 \%$ & $75.6 \%$ & $81.8 \%$ & $70.9 \%$ \\
\hline \multirow{2}{*}{$\begin{array}{l}\text { Rejuvenating } \\
\text { flake }\end{array}$} & count & 2 & 0 & 0 & 2 \\
\hline & $\%$ within raw material & $2.1 \%$ & $0.0 \%$ & $0.0 \%$ & $0.9 \%$ \\
\hline \multirow{2}{*}{ Total } & count & 96 & 90 & 44 & 230 \\
\hline & $\%$ within raw material & $100.0 \%$ & $100.0 \%$ & $100.0 \%$ & $100.0 \%$ \\
\hline
\end{tabular}


Lithic analysis of the Middle and Late Upper Palaeolithic in Hungary

Table 12. Arka knapped product frequency in tool assemblage by raw materials

\begin{tabular}{|c|c|c|c|c|c|c|c|}
\hline & \multicolumn{3}{|c|}{ Raw material } & \multirow{2}{*}{ Total } \\
\hline & & & & local & regional & transcarpathian & \\
\hline \multirow[t]{6}{*}{ Flake } & \multirow[t]{4}{*}{ state } & \multirow[t]{2}{*}{ blank } & count & 2051 & 255 & 87 & 2393 \\
\hline & & & $\%$ within raw material & $98.3 \%$ & $92.1 \%$ & $91.6 \%$ & $97.4 \%$ \\
\hline & & \multirow[t]{2}{*}{ tool } & count & 35 & 22 & 8 & 65 \\
\hline & & & $\%$ within raw material & $1.7 \%$ & $7.9 \%$ & $8.4 \%$ & $2.6 \%$ \\
\hline & \multirow{2}{*}{\multicolumn{2}{|c|}{ total }} & count & 2086 & 277 & 95 & 2458 \\
\hline & & & $\%$ within raw material & $100.0 \%$ & $100.0 \%$ & $100.0 \%$ & $100.0 \%$ \\
\hline \multirow[t]{6}{*}{ Blade } & \multirow[t]{4}{*}{ state } & \multirow[t]{2}{*}{ blank } & count & 1061 & 372 & 158 & 1591 \\
\hline & & & $\%$ within raw material & $94.7 \%$ & $84.5 \%$ & $81.4 \%$ & $90.7 \%$ \\
\hline & & \multirow[t]{2}{*}{ tool } & count & 59 & 68 & 36 & 163 \\
\hline & & & $\%$ within raw material & $5.3 \%$ & $15.5 \%$ & $18.6 \%$ & $9.3 \%$ \\
\hline & \multirow{2}{*}{\multicolumn{2}{|c|}{ total }} & count & 1120 & 440 & 194 & 1754 \\
\hline & & & $\%$ within raw material & $100.0 \%$ & $100.0 \%$ & $100.0 \%$ & $100.0 \%$ \\
\hline \multirow[t]{4}{*}{ Debris } & \multirow[t]{2}{*}{ state } & \multirow[t]{2}{*}{ blank } & count & 2612 & 207 & 59 & 2878 \\
\hline & & & \% within raw material & $100.0 \%$ & $100.0 \%$ & $100.0 \%$ & $100.0 \%$ \\
\hline & \multirow{2}{*}{\multicolumn{2}{|c|}{ total }} & count & 2612 & 207 & 59 & 2878 \\
\hline & & & $\%$ within raw material & $100.0 \%$ & $100.0 \%$ & $100.0 \%$ & $100.0 \%$ \\
\hline \multirow{6}{*}{$\begin{array}{l}\text { Rejuvenating } \\
\text { flake }\end{array}$} & \multirow[t]{4}{*}{ state } & \multirow[t]{2}{*}{ blank } & count & 63 & 12 & 4 & 79 \\
\hline & & & $\%$ within raw material & $96.9 \%$ & $100.0 \%$ & $100.0 \%$ & $97.5 \%$ \\
\hline & & \multirow[t]{2}{*}{ tool } & count & 2 & 0 & 0 & 2 \\
\hline & & & $\%$ within raw material & $3.1 \%$ & $0.0 \%$ & $0.0 \%$ & $2.5 \%$ \\
\hline & \multirow{2}{*}{\multicolumn{2}{|c|}{ total }} & count & 65 & 12 & 4 & 81 \\
\hline & & & \% within raw material & $100.0 \%$ & $100.0 \%$ & $100.0 \%$ & $100.0 \%$ \\
\hline
\end{tabular}

Table 13. Arka blade tool assemblage length, width and thickness by raw materials

\begin{tabular}{|c|c|c|c|c|}
\hline \multicolumn{2}{|l|}{ Raw material } & Length [mm] & Width [mm] & Thickness [mm] \\
\hline \multirow[t]{6}{*}{ Local } & Minimum & 10.10 & 4.20 & 1.80 \\
\hline & Maximum & 113.70 & 47.20 & 32.90 \\
\hline & Mean & 46.2464 & 20.3846 & 9.4027 \\
\hline & Median & 40.9000 & 19.7000 & 7.8000 \\
\hline & $\mathrm{N}$ & 59 & 59 & 59 \\
\hline & Std. deviation & 25.18068 & 9.59131 & 6.63280 \\
\hline \multirow[t]{6}{*}{ Regional } & Minimum & 7.60 & 3.60 & 1.00 \\
\hline & Maximum & 67.10 & 29.00 & 14.00 \\
\hline & Mean & 29.9347 & 13.2813 & 4.7479 \\
\hline & Median & 27.5000 & 13.1750 & 4.1000 \\
\hline & $\mathrm{N}$ & 68 & 68 & 68 \\
\hline & Std. deviation & 12.27628 & 5.91327 & 2.57142 \\
\hline \multirow[t]{6}{*}{ Transcarpathian } & Minimum & 9.10 & 3.68 & 1.48 \\
\hline & Maximum & 144.80 & 50.70 & 13.20 \\
\hline & Mean & 34.7197 & 14.1292 & 4.6394 \\
\hline & Median & 30.7000 & 12.1500 & 4.4000 \\
\hline & $\mathrm{N}$ & 36 & 36 & 36 \\
\hline & Std. deviation & 23.32726 & 9.12088 & 2.51161 \\
\hline \multirow[t]{6}{*}{ Total } & Minimum & 7.60 & 3.60 & 1.00 \\
\hline & Maximum & 144.80 & 50.70 & 32.90 \\
\hline & Mean & 36.8958 & 16.0397 & 6.4088 \\
\hline & Median & 32.6000 & 14.8000 & 5.2000 \\
\hline & $\mathrm{N}$ & 163 & 163 & 163 \\
\hline & Std. deviation & 21.45093 & 8.73217 & 4.99665 \\
\hline
\end{tabular}


Table 14. Arka blade blank and tool length, width and thickness mean t-test comparison by raw material

\begin{tabular}{|c|c|c|c|c|c|c|c|c|}
\hline \multicolumn{9}{|c|}{ Independent Samples Test } \\
\hline \multirow{2}{*}{\multicolumn{2}{|c|}{ Raw material }} & \multicolumn{2}{|c|}{$\begin{array}{c}\text { Levene's Test } \\
\text { for Equality } \\
\text { of Variances }\end{array}$} & \multicolumn{5}{|c|}{ t-test for Equality of Means } \\
\hline & & $\mathrm{F}$ & Sig. & $\mathrm{t}$ & $\mathrm{df}$ & \multirow{2}{*}{$\begin{array}{c}\begin{array}{c}\text { Sig. } \\
\text { (2-tailed) }\end{array} \\
0.001\end{array}$} & \multirow{2}{*}{$\begin{array}{c}\begin{array}{c}\text { Mean } \\
\text { difference }\end{array} \\
10.31465\end{array}$} & $\begin{array}{l}\text { Std. error } \\
\text { difference }\end{array}$ \\
\hline \multirow[t]{6}{*}{ Local } & \multirow{2}{*}{$\begin{array}{l}\text { length } \\
{[\mathrm{mm}]}\end{array}$} & 1.013 & 0.315 & 3.224 & 351 & & & 3.19903 \\
\hline & & & & 2.933 & 76.485 & 0.004 & 10.31465 & 3.51696 \\
\hline & \multirow{2}{*}{$\begin{array}{l}\text { width } \\
{[\mathrm{mm}]}\end{array}$} & 0.244 & 0.622 & 1.041 & 351 & 0.299 & 1.40059 & 1.34539 \\
\hline & & & & 1.027 & 81.907 & 0.307 & 1.40059 & 1.36370 \\
\hline & \multirow{2}{*}{$\begin{array}{l}\text { thickness } \\
{[\mathrm{mm}]}\end{array}$} & 5.489 & 0.020 & -1.323 & 351 & 0.187 & -0.97479 & 0.73681 \\
\hline & & & & -1.073 & 70.797 & 0.287 & -0.97479 & 0.90815 \\
\hline \multirow[t]{6}{*}{ Regional } & \multirow{2}{*}{$\begin{array}{l}\text { length } \\
{[\mathrm{mm}]}\end{array}$} & 0.703 & 0.403 & 4.133 & 157 & 0.000 & 9.07969 & 2.19708 \\
\hline & & & & 4.240 & 154.967 & 0.000 & 9.07969 & 2.14119 \\
\hline & \multirow{2}{*}{$\begin{array}{l}\text { width } \\
{[\mathrm{mm}]}\end{array}$} & 2.213 & 0.139 & 0.944 & 157 & 0.346 & 0.83450 & 0.88378 \\
\hline & & & & 0.927 & 133.523 & 0.356 & 0.83450 & 0.90049 \\
\hline & \multirow{2}{*}{$\begin{array}{l}\text { thickness } \\
{[\mathrm{mm}]}\end{array}$} & 0.382 & 0.537 & 1.620 & 157 & 0.107 & 0.68393 & 0.42218 \\
\hline & & & & 1.630 & 147.521 & 0.105 & 0.68393 & 0.41966 \\
\hline \multirow[t]{6}{*}{ Transcarpathian } & \multirow{2}{*}{$\begin{array}{l}\text { length } \\
{[\mathrm{mm}]}\end{array}$} & 0.771 & 0.382 & 2.213 & 109 & 0.029 & 7.83628 & 3.54032 \\
\hline & & & & 1.864 & 47.199 & 0.069 & 7.83628 & 4.20376 \\
\hline & \multirow{2}{*}{$\begin{array}{l}\text { width } \\
{[\mathrm{mm}]}\end{array}$} & 5.069 & 0.026 & 0.405 & 109 & 0.686 & 0.57883 & 1.42757 \\
\hline & & & & 0.348 & 49.045 & 0.729 & 0.57883 & 1.66126 \\
\hline & \multirow{2}{*}{$\begin{array}{l}\text { thickness } \\
{[\mathrm{mm}]}\end{array}$} & 0.201 & 0.655 & 0.053 & 109 & 0.958 & 0.02509 & 0.47569 \\
\hline & & & & 0.051 & 63.077 & 0.960 & 0.02509 & 0.49350 \\
\hline
\end{tabular}

Table 15. Arka blade tool length, width and thickness mean comparison by raw materials with ANOVA and the Tukey post hoc

ANOVA

\begin{tabular}{|l|l|r|r|r|r|c|}
\hline \multicolumn{2}{|c|}{} & Sum of squares & df & Mean square & F & Sig. \\
\hline \multirow{2}{*}{ Length $[\mathrm{mm}]$} & between groups & 8624.170 & 2 & 4312.085 & 10.466 & 0.000 \\
\cline { 2 - 7 } & within groups & 65918.883 & 160 & 411.993 & & \\
\cline { 2 - 7 } & Total & 74543.053 & 162 & & & \\
\hline \multirow{3}{*}{ Width $[\mathrm{mm}]$} & between groups & 1762.592 & 2 & 881.296 & 13.315 & 0.000 \\
\cline { 2 - 7 } & within groups & 10590.045 & 160 & 66.188 & & \\
\cline { 2 - 7 } & Total & 12352.636 & 162 & & & \\
\hline \multirow{2}{*}{ Thickness [mm] } & between groups & 829.124 & 2 & 414.562 & 20.628 & 0.000 \\
\cline { 2 - 7 } & within groups & 3215.457 & 160 & 20.097 & & \\
\cline { 2 - 7 } & Total & 4044.581 & 162 & & & \\
\hline
\end{tabular}


Table 15. Continued

Multiple Comparisons

Tukey HSD

\begin{tabular}{|c|c|c|c|c|c|c|c|}
\hline \multirow{2}{*}{$\begin{array}{l}\text { Dependent } \\
\text { variable }\end{array}$} & \multirow{2}{*}{ (I) raw material } & \multirow{2}{*}{ (J) raw material } & \multirow{2}{*}{$\begin{array}{c}\text { Mean } \\
\text { difference } \\
\quad(\mathrm{I}-\mathrm{J})\end{array}$} & \multirow{2}{*}{$\begin{array}{l}\text { Std. } \\
\text { error }\end{array}$} & \multirow{2}{*}{ Sig. } & \multicolumn{2}{|c|}{$\begin{array}{l}95 \% \text { Confidence } \\
\text { interval }\end{array}$} \\
\hline & & & & & & $\begin{array}{l}\text { Lower } \\
\text { bound }\end{array}$ & $\begin{array}{l}\text { Upper } \\
\text { bound }\end{array}$ \\
\hline \multirow{6}{*}{$\begin{array}{l}\text { Length } \\
{[\mathrm{mm}]}\end{array}$} & \multirow[t]{2}{*}{ local } & regional & 16.31173 & 3.61132 & 0.000 & 7.7683 & 24.8551 \\
\hline & & transcarpathian & 11.52672 & 4.29269 & 0.022 & 1.3714 & 21.6821 \\
\hline & \multirow[t]{2}{*}{ regional } & local & -16.31173 & 3.61132 & 0.000 & -24.8551 & -7.7683 \\
\hline & & transcarpathian & -4.78502 & 4.18366 & 0.489 & -14.6824 & 5.1124 \\
\hline & \multirow[t]{2}{*}{ transcarpathian } & local & -11.52672 & 4.29269 & 0.022 & -21.6821 & -1.3714 \\
\hline & & regional & 4.78502 & 4.18366 & 0.489 & -5.1124 & 14.6824 \\
\hline \multirow{6}{*}{$\begin{array}{l}\text { Width } \\
{[\mathrm{mm}]}\end{array}$} & \multirow[t]{2}{*}{ local } & regional & 7.10325 & 1.44747 & 0.000 & 3.6789 & 10.5276 \\
\hline & & transcarpathian & 6.25541 & 1.72057 & 0.001 & 2.1850 & 10.3258 \\
\hline & \multirow[t]{2}{*}{ regional } & local & -7.10325 & 1.44747 & 0.000 & -10.5276 & -3.6789 \\
\hline & & transcarpathian & -0.84784 & 1.67687 & 0.869 & -4.8149 & 3.1192 \\
\hline & \multirow[t]{2}{*}{ transcarpathian } & local & -6.25541 & 1.72057 & 0.001 & -10.3258 & -2.1850 \\
\hline & & regional & 0.84784 & 1.67687 & 0.869 & -3.1192 & 4.8149 \\
\hline \multirow{6}{*}{$\begin{array}{l}\text { Thickness } \\
{[\mathrm{mm}]}\end{array}$} & \multirow[t]{2}{*}{ local } & regional & 4.65477 & 0.79760 & 0.000 & 2.7679 & 6.5417 \\
\hline & & transcarpathian & 4.76327 & 0.94808 & 0.000 & 2.5204 & 7.0062 \\
\hline & \multirow[t]{2}{*}{ regional } & local & -4.65477 & 0.79760 & 0.000 & -6.5417 & -2.7679 \\
\hline & & transcarpathian & 0.10850 & 0.92400 & 0.992 & -2.0774 & 2.2944 \\
\hline & \multirow[t]{2}{*}{ transcarpathian } & local & -4.76327 & 0.94808 & 0.000 & -7.0062 & -2.5204 \\
\hline & & regional & -0.10850 & 0.92400 & 0.992 & -2.2944 & 2.0774 \\
\hline
\end{tabular}

Table 16. Arka blade tool types by raw material

\begin{tabular}{|c|c|c|c|c|c|c|}
\hline & \multicolumn{3}{|c|}{ Raw material } & \multirow{2}{*}{ Total } \\
\hline & & & local & regional & transcarpathian & \\
\hline \multirow[t]{18}{*}{ Tooltypes } & \multirow[t]{2}{*}{ endscraper } & count & 23 & 8 & 9 & 40 \\
\hline & & $\%$ within raw material & $39.0 \%$ & $11.8 \%$ & $25.0 \%$ & $24.5 \%$ \\
\hline & \multirow[t]{2}{*}{ burin } & count & 10 & 6 & 0 & 16 \\
\hline & & $\%$ within raw material & $16.9 \%$ & $8.8 \%$ & $0.0 \%$ & $9.8 \%$ \\
\hline & \multirow[t]{2}{*}{ retouched } & count & 4 & 15 & 7 & 26 \\
\hline & & $\%$ within raw material & $6.8 \%$ & $22.1 \%$ & $19.4 \%$ & $16.0 \%$ \\
\hline & \multirow[t]{2}{*}{ borer } & count & 0 & 0 & 1 & 1 \\
\hline & & \% within raw material & $0.0 \%$ & $0.0 \%$ & $2.8 \%$ & $0.6 \%$ \\
\hline & \multirow{2}{*}{$\begin{array}{l}\text { splintered } \\
\text { piece }\end{array}$} & count & 1 & 0 & 0 & 1 \\
\hline & & \% within raw material & $1.7 \%$ & $0.0 \%$ & $0.0 \%$ & $0.6 \%$ \\
\hline & \multirow{2}{*}{ truncation } & count & 6 & 12 & 5 & 23 \\
\hline & & $\%$ within raw material & $10.2 \%$ & $17.6 \%$ & $13.9 \%$ & $14.1 \%$ \\
\hline & \multirow{2}{*}{$\begin{array}{l}\text { notched- } \\
\text { denticulated }\end{array}$} & count & 2 & 2 & 0 & 4 \\
\hline & & $\%$ within raw material & $3.4 \%$ & $2.9 \%$ & $0.0 \%$ & $2.5 \%$ \\
\hline & \multirow[t]{2}{*}{ composite } & count & 1 & 1 & 1 & 3 \\
\hline & & $\%$ within raw material & $1.7 \%$ & $1.5 \%$ & $2.8 \%$ & $1.8 \%$ \\
\hline & \multirow[t]{2}{*}{ armature } & count & 12 & 24 & 13 & 49 \\
\hline & & $\%$ within raw material & $20.3 \%$ & $35.3 \%$ & $36.1 \%$ & $30.1 \%$ \\
\hline \multirow{2}{*}{\multicolumn{2}{|c|}{ Total }} & count & 59 & 68 & 36 & 163 \\
\hline & & $\%$ within raw material & $100.0 \%$ & $100.0 \%$ & $100.0 \%$ & $100.0 \%$ \\
\hline
\end{tabular}


Table 17. Arka armature types by raw material

\begin{tabular}{|c|c|c|c|c|c|c|}
\hline & \multicolumn{3}{|c|}{ Raw material } & \multirow{2}{*}{ Total } \\
\hline & & & local & regional & transcarpathian & \\
\hline \multirow[t]{10}{*}{ Armatures } & \multirow[t]{2}{*}{ backed } & count & 2 & 15 & 7 & 24 \\
\hline & & $\%$ within raw material & $16.7 \%$ & $62.5 \%$ & $53.8 \%$ & $49.0 \%$ \\
\hline & \multirow{2}{*}{$\begin{array}{l}\text { backed- } \\
\text { truncated }\end{array}$} & count & 7 & 1 & 3 & 11 \\
\hline & & $\%$ within raw material & $58.3 \%$ & $4.2 \%$ & $23.1 \%$ & $22.4 \%$ \\
\hline & \multirow{2}{*}{$\begin{array}{l}\text { backed- } \\
\text { ventral truncation }\end{array}$} & count & 0 & 1 & 0 & 1 \\
\hline & & $\%$ within raw material & $0.0 \%$ & $4.2 \%$ & $0.0 \%$ & $2.0 \%$ \\
\hline & \multirow[t]{2}{*}{ trapeze-rectangle } & count & 0 & 0 & 1 & 1 \\
\hline & & $\%$ within raw material & $0.0 \%$ & $0.0 \%$ & $7.7 \%$ & $2.0 \%$ \\
\hline & \multirow[t]{2}{*}{ points } & count & 3 & 7 & 2 & 12 \\
\hline & & $\%$ within raw material & $25.0 \%$ & $29.2 \%$ & $15.4 \%$ & $24.5 \%$ \\
\hline \multirow{2}{*}{\multicolumn{2}{|c|}{ Total }} & count & 12 & 24 & 13 & 49 \\
\hline & & $\%$ within raw material & $100.0 \%$ & $100.0 \%$ & $100.0 \%$ & $100.0 \%$ \\
\hline
\end{tabular}

Table 18. Arka point types by raw material

\begin{tabular}{|c|c|c|c|c|c|c|}
\hline & \multicolumn{3}{|c|}{ Raw material } & \multirow{2}{*}{ Total } \\
\hline & & & local & regional & transcarpathian & \\
\hline \multirow[t]{8}{*}{ Points } & \multirow[t]{2}{*}{ retouched } & count & 3 & 2 & 1 & 6 \\
\hline & & $\%$ within raw material & $100.0 \%$ & $28.6 \%$ & $50.0 \%$ & $50.0 \%$ \\
\hline & \multirow{2}{*}{$\begin{array}{l}\text { gravette/ } \\
\text { microgravette }\end{array}$} & count & 0 & 3 & 1 & 4 \\
\hline & & $\%$ within raw material & $0.0 \%$ & $42.9 \%$ & $50.0 \%$ & $33.3 \%$ \\
\hline & \multirow[t]{2}{*}{ backed } & count & 0 & 1 & 0 & 1 \\
\hline & & $\%$ within raw material & $0.0 \%$ & $14.3 \%$ & $0.0 \%$ & $8.3 \%$ \\
\hline & \multirow[t]{2}{*}{ fléchette } & count & 0 & 1 & 0 & 1 \\
\hline & & $\%$ within raw material & $0.0 \%$ & $14.3 \%$ & $0.0 \%$ & $8.3 \%$ \\
\hline \multirow{2}{*}{\multicolumn{2}{|c|}{ Total }} & count & 3 & 7 & 2 & 12 \\
\hline & & $\%$ within raw material & $100.0 \%$ & $100.0 \%$ & $100.0 \%$ & $100.0 \%$ \\
\hline
\end{tabular}

Table 19. Arka blank flake assemblage dorsal scar pattern

\begin{tabular}{|c|c|c|c|c|c|c|}
\hline & \multicolumn{3}{|c|}{ Raw material } & \multirow{2}{*}{ Total } \\
\hline & & & local & regional & transcarpathian & \\
\hline \multirow[t]{10}{*}{ Scars } & \multirow[t]{2}{*}{ unidirectional } & count & 281 & 41 & 23 & 345 \\
\hline & & $\%$ within raw material & $35.4 \%$ & $44.1 \%$ & $52.3 \%$ & $37.1 \%$ \\
\hline & \multirow[t]{2}{*}{ opposite } & count & 101 & 8 & 3 & 112 \\
\hline & & $\%$ within raw material & $12.7 \%$ & $8.6 \%$ & $6.8 \%$ & $12.0 \%$ \\
\hline & \multirow[t]{2}{*}{ perpendicular } & count & 264 & 25 & 12 & 301 \\
\hline & & $\%$ within raw material & $33.3 \%$ & $26.9 \%$ & $27.3 \%$ & $32.4 \%$ \\
\hline & \multirow[t]{2}{*}{ multiple } & count & 141 & 7 & 4 & 152 \\
\hline & & $\%$ within raw material & $17.8 \%$ & $7.5 \%$ & $9.1 \%$ & $16.3 \%$ \\
\hline & \multirow[t]{2}{*}{ no scar } & count & 6 & 12 & 2 & 20 \\
\hline & & $\%$ within raw material & $0.8 \%$ & $12.9 \%$ & $4.5 \%$ & $2.2 \%$ \\
\hline \multirow{2}{*}{\multicolumn{2}{|c|}{ Total }} & count & 793 & 93 & 44 & 930 \\
\hline & & $\%$ within raw material & $100.0 \%$ & $100.0 \%$ & $100.0 \%$ & $100.0 \%$ \\
\hline
\end{tabular}


Table 20. Arka blank flake assemblage platform types

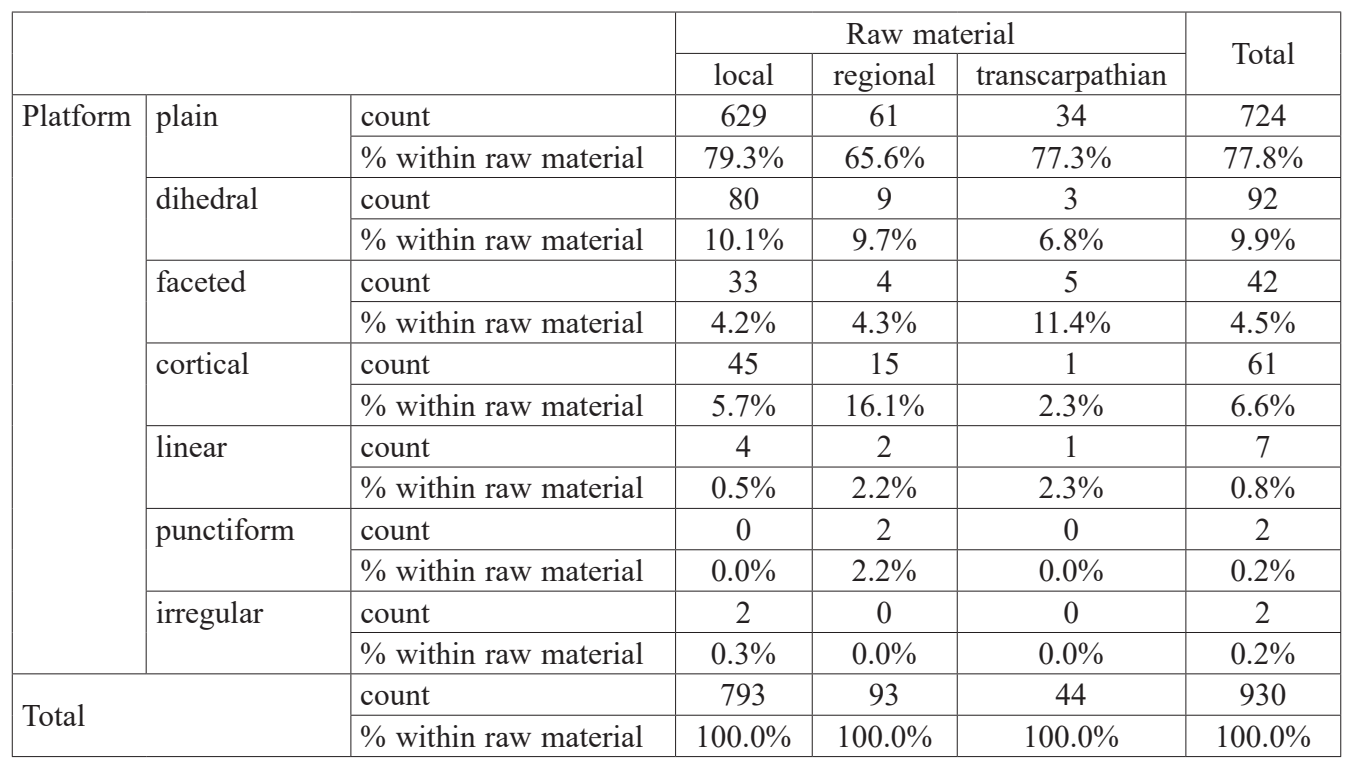

Table 21. Arka blank flake assemblage impact point-overhang frequency

\begin{tabular}{|c|c|c|c|c|c|c|}
\hline & \multicolumn{3}{|c|}{ Raw material } & \multirow{2}{*}{ Total } \\
\hline & & & local & regional & transcarpathian & \\
\hline \multirow{8}{*}{$\begin{array}{l}\text { Impact point- } \\
\text { overhang }\end{array}$} & \multirow[t]{2}{*}{ none } & count & 82 & 16 & 12 & 110 \\
\hline & & $\%$ within raw material & $10.3 \%$ & $17.2 \%$ & $27.3 \%$ & $11.8 \%$ \\
\hline & \multirow[t]{2}{*}{ yes-no } & count & 52 & 13 & 8 & 73 \\
\hline & & $\%$ within raw material & $6.6 \%$ & $14.0 \%$ & $18.2 \%$ & $7.8 \%$ \\
\hline & \multirow[t]{2}{*}{ yes-yes } & count & 418 & 38 & 10 & 466 \\
\hline & & $\%$ within raw material & $52.7 \%$ & $40.9 \%$ & $22.7 \%$ & $50.1 \%$ \\
\hline & \multirow[t]{2}{*}{ no-yes } & count & 241 & 26 & 14 & 281 \\
\hline & & $\%$ within raw material & $30.4 \%$ & $28.0 \%$ & $31.8 \%$ & $30.2 \%$ \\
\hline \multirow{2}{*}{\multicolumn{2}{|c|}{ Total }} & count & 793 & 93 & 44 & 930 \\
\hline & & $\%$ within raw material & $100.0 \%$ & $100.0 \%$ & $100.0 \%$ & $100.0 \%$ \\
\hline
\end{tabular}


Table 22. Arka flake assemblage length, width and thickness by raw materials

\begin{tabular}{|c|c|c|c|c|}
\hline \multicolumn{2}{|l|}{ Raw material } & Length [mm] & Width [mm] & Thickness [mm] \\
\hline \multirow[t]{6}{*}{ Local } & Minimum & 14.50 & 13.60 & 1.80 \\
\hline & Maximum & 140.10 & 127.00 & 75.00 \\
\hline & Mean & 50.2058 & 42.4666 & 12.9763 \\
\hline & Median & 47.1000 & 38.8000 & 11.6000 \\
\hline & $\mathrm{N}$ & 793 & 793 & 793 \\
\hline & Std. deviation & 19.03758 & 18.42058 & 7.39474 \\
\hline \multirow[t]{6}{*}{ Regional } & Minimum & 10.70 & 9.40 & 2.00 \\
\hline & Maximum & 95.00 & 49.50 & 29.00 \\
\hline & Mean & 31.3548 & 25.3937 & 8.3366 \\
\hline & Median & 27.2000 & 24.4000 & 7.1000 \\
\hline & $\mathrm{N}$ & 93 & 93 & 93 \\
\hline & Std. deviation & 14.04941 & 9.70546 & 4.86216 \\
\hline \multirow[t]{6}{*}{ Transcarpathian } & Minimum & 14.30 & 12.50 & 2.60 \\
\hline & Maximum & 71.00 & 81.00 & 16.80 \\
\hline & Mean & 37.7500 & 28.0205 & 6.7136 \\
\hline & Median & 34.7000 & 24.8000 & 6.2500 \\
\hline & $\mathrm{N}$ & 44 & 44 & 44 \\
\hline & Std. deviation & 13.43672 & 13.20801 & 3.08896 \\
\hline \multirow[t]{6}{*}{ Total } & Minimum & 10.70 & 9.40 & 1.80 \\
\hline & Maximum & 140.10 & 127.00 & 75.00 \\
\hline & Mean & 47.7314 & 40.0758 & 12.2161 \\
\hline & Median & 44.6500 & 36.2000 & 10.4500 \\
\hline & $\mathrm{N}$ & 930 & 930 & 930 \\
\hline & Std. deviation & 19.33078 & 18.43973 & 7.26878 \\
\hline
\end{tabular}

Table 23. Arka flake length, width and thickness mean comparison by raw materials with ANOVA and the Tukey post hoc

ANOVA

\begin{tabular}{|l|l|r|r|r|r|c|}
\hline \multicolumn{2}{|c|}{} & Sum of squares & df & Mean square & \multicolumn{1}{c|}{ F } & Sig. \\
\hline \multirow{4}{*}{ Length $[\mathrm{mm}]$} & between groups & 34180.750 & 2 & 17090.375 & 50.621 & 0.000 \\
\cline { 2 - 7 } & within groups & 312967.134 & 927 & 337.613 & & \\
\cline { 2 - 7 } & Total & 347147.883 & 929 & & & \\
\hline \multirow{4}{*}{ Width $[\mathrm{mm}]$} & between groups & 30974.915 & 2 & 15487.458 & 50.391 & 0.000 \\
\cline { 2 - 7 } & within groups & 284907.130 & 927 & 307.343 & & \\
\cline { 2 - 7 } & Total & 315882.046 & 929 & & & \\
\hline \multirow{3}{*}{ Thickness [mm] } & between groups & 3190.250 & 2 & 1595.125 & 32.220 & 0.000 \\
\cline { 2 - 7 } & within groups & 45893.549 & 927 & 49.508 & & \\
\cline { 2 - 7 } & Total & 49083.799 & 929 & & & \\
\hline
\end{tabular}


Table 23. Continued

Multiple Comparisons

Tukey HSD

\begin{tabular}{|c|c|c|c|c|c|c|c|}
\hline \multirow{2}{*}{$\begin{array}{l}\text { Dependent } \\
\text { variable }\end{array}$} & \multirow{2}{*}{ (I) raw material } & \multirow{2}{*}{ (J) raw material } & \multirow{2}{*}{$\begin{array}{c}\text { Mean } \\
\text { difference } \\
(\mathrm{I}-\mathrm{J})\end{array}$} & \multirow{2}{*}{$\begin{array}{l}\text { Std. } \\
\text { error }\end{array}$} & \multirow{2}{*}{ Sig. } & \multicolumn{2}{|c|}{$\begin{array}{l}95 \% \text { Confidence } \\
\text { interval }\end{array}$} \\
\hline & & & & & & $\begin{array}{l}\text { Lower } \\
\text { bound }\end{array}$ & $\begin{array}{l}\text { Upper } \\
\text { bound }\end{array}$ \\
\hline \multirow{6}{*}{$\begin{array}{l}\text { Length } \\
{[\mathrm{mm}]}\end{array}$} & \multirow[t]{2}{*}{ local } & regional & 18.85096 & 2.01395 & 0.000 & 14.1233 & 23.5787 \\
\hline & & transcarpathian & 12.45580 & 2.84583 & 0.000 & 5.7753 & 19.1363 \\
\hline & \multirow[t]{2}{*}{ regional } & local & -18.85096 & 2.01395 & 0.000 & -23.5787 & -14.1233 \\
\hline & & transcarpathian & -6.39516 & 3.36203 & 0.139 & -14.2875 & 1.4971 \\
\hline & \multirow[t]{2}{*}{ transcarpathian } & local & -12.45580 & 2.84583 & 0.000 & -19.1363 & -5.7753 \\
\hline & & regional & 6.39516 & 3.36203 & 0.139 & -1.4971 & 14.2875 \\
\hline \multirow{6}{*}{$\begin{array}{l}\text { Width } \\
{[\mathrm{mm}]}\end{array}$} & \multirow[t]{2}{*}{ local } & regional & 17.07295 & 1.92155 & 0.000 & 12.5622 & 21.5837 \\
\hline & & transcarpathian & 14.44615 & 2.71526 & 0.000 & 8.0721 & 20.8202 \\
\hline & \multirow[t]{2}{*}{ regional } & local & -17.07295 & 1.92155 & 0.000 & -21.5837 & -12.5622 \\
\hline & & transcarpathian & -2.62680 & 3.20778 & 0.691 & -10.1570 & 4.9034 \\
\hline & \multirow[t]{2}{*}{ transcarpathian } & local & -14.44615 & 2.71526 & 0.000 & -20.8202 & -8.0721 \\
\hline & & regional & 2.62680 & 3.20778 & 0.691 & -4.9034 & 10.1570 \\
\hline \multirow{6}{*}{$\begin{array}{l}\text { Thickness } \\
{[\mathrm{mm}]}\end{array}$} & \multirow[t]{2}{*}{ local } & regional & 4.63978 & 0.77121 & 0.000 & 2.8294 & 6.4502 \\
\hline & & transcarpathian & 6.26271 & 1.08977 & 0.000 & 3.7045 & 8.8209 \\
\hline & \multirow[t]{2}{*}{ regional } & local & -4.63978 & 0.77121 & 0.000 & -6.4502 & -2.8294 \\
\hline & & transcarpathian & 1.62292 & 1.28744 & 0.418 & -1.3993 & 4.6452 \\
\hline & \multirow[t]{2}{*}{\begin{tabular}{|l|} 
transcarpathian \\
\end{tabular}} & local & -6.26271 & 1.08977 & 0.000 & -8.8209 & -3.7045 \\
\hline & & regional & -1.62292 & 1.28744 & 0.418 & -4.6452 & 1.3993 \\
\hline
\end{tabular}

Table 24. Arka flake tool length, width and thickness by raw material

\begin{tabular}{|c|c|c|c|c|}
\hline \multicolumn{2}{|l|}{ Raw material } & Length [mm] & Width [mm] & Thickness [mm] \\
\hline \multirow[t]{6}{*}{ Local } & Minimum & 18.40 & 15.50 & 4.90 \\
\hline & Maximum & 129.20 & 62.20 & 43.00 \\
\hline & Mean & 46.4654 & 33.9463 & 14.7206 \\
\hline & Median & 41.4000 & 32.8000 & 10.7000 \\
\hline & $\mathrm{N}$ & 35 & 35 & 35 \\
\hline & Std. deviation & 22.05995 & 10.94052 & 9.65583 \\
\hline \multirow[t]{6}{*}{ Regional } & Minimum & 16.40 & 11.30 & 3.90 \\
\hline & Maximum & 71.00 & 47.90 & 16.20 \\
\hline & Mean & 35.0545 & 26.1682 & 10.7091 \\
\hline & Median & 32.3000 & 25.4000 & 10.6000 \\
\hline & $\mathrm{N}$ & 22 & 22 & 22 \\
\hline & Std. deviation & 12.76475 & 9.01789 & 3.67422 \\
\hline \multirow[t]{6}{*}{ Transcarpathian } & Minimum & 23.00 & 13.20 & 3.80 \\
\hline & Maximum & 51.20 & 33.80 & 19.90 \\
\hline & Mean & 32.7250 & 21.9750 & 10.5250 \\
\hline & Median & 30.2000 & 20.3500 & 10.0000 \\
\hline & $\mathrm{N}$ & 8 & 8 & 8 \\
\hline & Std. deviation & 9.00869 & 7.87759 & 4.99535 \\
\hline \multirow[t]{6}{*}{ Total } & Minimum & 16.40 & 11.30 & 3.80 \\
\hline & Maximum & 129.20 & 62.20 & 43.00 \\
\hline & Mean & 40.9122 & 29.8403 & 12.8465 \\
\hline & Median & 37.5000 & 29.3500 & 10.7000 \\
\hline & $\mathrm{N}$ & 65 & 65 & 65 \\
\hline & Std. deviation & 18.91844 & 10.89253 & 7.80095 \\
\hline
\end{tabular}


Table 25. Arka flake blank and tool length, width and thickness mean t-test comparison by raw material

\begin{tabular}{|c|c|c|c|c|c|c|c|c|}
\hline \multicolumn{9}{|c|}{ Independent Samples Test } \\
\hline \multirow{2}{*}{\multicolumn{2}{|c|}{ Raw material }} & \multicolumn{2}{|c|}{$\begin{array}{c}\text { Levene's Test } \\
\text { for Equality } \\
\text { of Variances }\end{array}$} & \multicolumn{5}{|c|}{ t-test for Equality of Means } \\
\hline & & $\mathrm{F}$ & Sig. & $\mathrm{t}$ & df & $\begin{array}{c}\text { Sig. } \\
\text { (2-tailed) }\end{array}$ & $\begin{array}{c}\text { Mean } \\
\text { difference }\end{array}$ & $\begin{array}{l}\text { Std. error } \\
\text { difference }\end{array}$ \\
\hline \multirow[t]{6}{*}{ Local } & \multirow{2}{*}{$\begin{array}{l}\text { length } \\
{[\mathrm{mm}]}\end{array}$} & 0.026 & 0.871 & 1.130 & 826 & 0.259 & 3.74037 & 3.31130 \\
\hline & & & & 0.987 & 36.270 & 0.330 & 3.74037 & 3.78960 \\
\hline & \multirow{2}{*}{$\begin{array}{l}\text { width } \\
{[\mathrm{mm}]}\end{array}$} & 7.560 & 0.006 & 2.714 & 826 & 0.007 & 8.52032 & 3.13895 \\
\hline & & & & 4.344 & 43.011 & 0.000 & 8.52032 & 1.96157 \\
\hline & \multirow{2}{*}{$\begin{array}{l}\text { thickness } \\
{[\mathrm{mm}]}\end{array}$} & 4.534 & 0.034 & -1.346 & 826 & 0.179 & -1.74423 & 1.29563 \\
\hline & & & & -1.055 & 35.782 & 0.298 & -1.74423 & 1.65312 \\
\hline \multirow[t]{6}{*}{ Regional } & \multirow{2}{*}{$\begin{array}{l}\text { length } \\
{[\mathrm{mm}]}\end{array}$} & 0.058 & 0.811 & -1.129 & 113 & 0.261 & -3.69971 & 3.27638 \\
\hline & & & & -1.199 & 34.121 & 0.239 & -3.69971 & 3.08687 \\
\hline & \multirow{2}{*}{$\begin{array}{l}\text { width } \\
\text { [mm] }\end{array}$} & 0.454 & 0.502 & -0.341 & 113 & 0.734 & -0.77453 & 2.27157 \\
\hline & & & & -0.357 & 33.511 & 0.723 & -0.77453 & 2.17010 \\
\hline & \multirow{2}{*}{$\begin{array}{l}\text { thickness } \\
{[\mathrm{mm}]}\end{array}$} & 1.487 & 0.225 & -2.145 & 113 & 0.034 & -2.37253 & 1.10582 \\
\hline & & & & -2.547 & 40.419 & 0.015 & -2.37253 & 0.93158 \\
\hline \multirow[t]{6}{*}{ Transcarpathian } & \multirow{2}{*}{$\begin{array}{l}\text { length } \\
{[\mathrm{mm}]}\end{array}$} & 2.096 & 0.154 & 1.013 & 50 & 0.316 & 5.02500 & 4.96144 \\
\hline & & & & 1.331 & 13.450 & 0.205 & 5.02500 & 3.77463 \\
\hline & \multirow{2}{*}{$\begin{array}{l}\text { width } \\
{[\mathrm{mm}]}\end{array}$} & 1.032 & 0.315 & 1.248 & 50 & 0.218 & 6.04545 & 4.84218 \\
\hline & & & & 1.766 & 15.332 & 0.097 & 6.04545 & 3.42372 \\
\hline & \multirow{2}{*}{$\begin{array}{l}\text { thickness } \\
{[\mathrm{mm}]}\end{array}$} & 2.825 & 0.099 & -2.899 & 50 & 0.006 & -3.81136 & 1.31465 \\
\hline & & & & -2.087 & 8.001 & 0.070 & -3.81136 & 1.82649 \\
\hline
\end{tabular}

Table 26. Arka flake tool length, width and thickness mean comparison by raw materials with ANOVA and the Tukey post hoc

ANOVA

\begin{tabular}{|l|l|c|r|r|r|c|}
\hline \multicolumn{2}{|c|}{} & Sum of squares & df & Mean square & F & Sig. \\
\hline \multirow{3}{*}{ Length $[\mathrm{mm}]$} & between groups & 2370.451 & 2 & 1185.225 & 3.578 & 0.034 \\
\cline { 2 - 7 } & within groups & 20535.618 & 62 & 331.220 & & \\
\cline { 2 - 7 } & Total & 22906.068 & 64 & & & \\
\hline \multirow{3}{*}{ Width $[\mathrm{mm}]$} & between groups & 1381.631 & 2 & 690.815 & 6.895 & 0.002 \\
\cline { 2 - 7 } & within groups & 6211.793 & 62 & 100.190 & & \\
\cline { 2 - 7 } & Total & 7593.424 & 64 & & & \\
\hline \multirow{3}{*}{ Thickness [mm] } & between groups & 266.547 & 2 & 133.274 & 2.277 & 0.111 \\
\cline { 2 - 7 } & within groups & 3628.165 & 62 & 58.519 & & \\
\cline { 2 - 7 } & Total & 3894.712 & 64 & & & \\
\hline
\end{tabular}


Table 26. Continued

Multiple Comparisons

Tukey HSD

\begin{tabular}{|c|c|c|c|c|c|c|c|}
\hline \multirow{2}{*}{$\begin{array}{l}\text { Dependent } \\
\text { variable }\end{array}$} & \multirow{2}{*}{ (I) raw material } & \multirow{2}{*}{ (J) raw material } & \multirow{2}{*}{$\begin{array}{c}\text { Mean } \\
\text { difference } \\
\quad(\mathrm{I}-\mathrm{J})\end{array}$} & \multirow{2}{*}{$\begin{array}{l}\text { Std. } \\
\text { error }\end{array}$} & \multirow{2}{*}{ Sig. } & \multicolumn{2}{|c|}{$\begin{array}{l}\text { 95\% Confidence } \\
\text { interval }\end{array}$} \\
\hline & & & & & & $\begin{array}{l}\text { Lower } \\
\text { bound }\end{array}$ & $\begin{array}{l}\text { Upper } \\
\text { bound }\end{array}$ \\
\hline \multirow{6}{*}{$\begin{array}{l}\text { Length } \\
{[\mathrm{mm}]}\end{array}$} & \multirow[t]{2}{*}{ local } & regional & 11.41088 & 4.95165 & 0.063 & -0.4793 & 23.3011 \\
\hline & & transcarpathian & 13.74043 & 7.13203 & 0.140 & -3.3854 & 30.8663 \\
\hline & \multirow[t]{2}{*}{ regional } & local & -11.41088 & 4.95165 & 0.063 & -23.3011 & 0.4793 \\
\hline & & transcarpathian & 2.32955 & 7.51385 & 0.948 & -15.7131 & 20.3722 \\
\hline & \multirow[t]{2}{*}{ transcarpathian } & local & -13.74043 & 7.13203 & 0.140 & -30.8663 & 3.3854 \\
\hline & & regional & -2.32955 & 7.51385 & 0.948 & -20.3722 & 15.7131 \\
\hline \multirow{6}{*}{$\begin{array}{l}\text { Width } \\
{[\mathrm{mm}]}\end{array}$} & \multirow[t]{2}{*}{ local } & regional & 7.77810 & 2.72336 & 0.016 & 1.2386 & 14.3176 \\
\hline & & transcarpathian & 11.97129 & 3.92254 & 0.009 & 2.5522 & 21.3903 \\
\hline & \multirow[t]{2}{*}{ regional } & local & -7.77810 & 2.72336 & 0.016 & -14.3176 & -1.2386 \\
\hline & & transcarpathian & 4.19318 & 4.13254 & 0.570 & -5.7301 & 14.1165 \\
\hline & \multirow[t]{2}{*}{ transcarpathian } & local & -11.97129 & 3.92254 & 0.009 & -21.3903 & -2.5522 \\
\hline & & regional & -4.19318 & 4.13254 & 0.570 & -14.1165 & 5.7301 \\
\hline \multirow{6}{*}{$\begin{array}{l}\text { Thickness } \\
{[\mathrm{mm}]}\end{array}$} & \multirow[t]{2}{*}{ local } & regional & 4.01148 & 2.08132 & 0.140 & -0.9863 & 9.0093 \\
\hline & & transcarpathian & 4.19557 & 2.99780 & 0.347 & -3.0029 & 11.3941 \\
\hline & \multirow[t]{2}{*}{ regional } & local & -4.01148 & 2.08132 & 0.140 & -9.0093 & 0.9863 \\
\hline & & transcarpathian & 0.18409 & 3.15829 & 0.998 & -7.3998 & 7.7680 \\
\hline & \multirow[t]{2}{*}{ transcarpathian } & local & -4.19557 & 2.99780 & 0.347 & -11.3941 & 3.0029 \\
\hline & & regional & -0.18409 & 3.15829 & 0.998 & -7.7680 & 7.3998 \\
\hline
\end{tabular}

Table 27. Arka flake tool types by raw material

\begin{tabular}{|c|c|c|c|c|c|c|}
\hline & \multicolumn{3}{|c|}{ Raw material } & \multirow{2}{*}{ Total } \\
\hline & & & local & regional & transcarpathian & \\
\hline \multirow[t]{12}{*}{ Tooltypes } & \multirow[t]{2}{*}{ endscraper } & count & 12 & 15 & 3 & 30 \\
\hline & & $\%$ within raw material & $34.3 \%$ & $68.2 \%$ & $37.5 \%$ & $46.2 \%$ \\
\hline & \multirow[t]{2}{*}{ burin } & count & 8 & 3 & 3 & 14 \\
\hline & & $\%$ within raw material & $22.9 \%$ & $13.6 \%$ & $37.5 \%$ & $21.5 \%$ \\
\hline & \multirow[t]{2}{*}{ retouched } & count & 6 & 3 & 2 & 11 \\
\hline & & $\%$ within raw material & $17.1 \%$ & $13.6 \%$ & $25.0 \%$ & $16.9 \%$ \\
\hline & \multirow[t]{2}{*}{ borer } & count & 1 & 0 & 0 & 1 \\
\hline & & $\%$ within raw material & $2.9 \%$ & $0.0 \%$ & $0.0 \%$ & $1.5 \%$ \\
\hline & \multirow[t]{2}{*}{ composite } & count & 1 & 0 & 0 & 1 \\
\hline & & $\%$ within raw material & $2.9 \%$ & $0.0 \%$ & $0.0 \%$ & $1.5 \%$ \\
\hline & \multirow{2}{*}{$\begin{array}{l}\text { notched- } \\
\text { denticulated }\end{array}$} & count & 7 & 1 & 0 & 8 \\
\hline & & $\%$ within raw material & $20.0 \%$ & $4.5 \%$ & $0.0 \%$ & $12.3 \%$ \\
\hline \multirow{2}{*}{\multicolumn{2}{|c|}{ Total }} & count & 35 & 22 & 8 & 65 \\
\hline & & $\%$ within raw material & $100.0 \%$ & $100.0 \%$ & $100.0 \%$ & $100.0 \%$ \\
\hline
\end{tabular}




\section{BODROGKERESZTÚR}

The site

The site was found on a hill (named Henye) $190 \mathrm{~m}$ a.s.l. at the village Bodrogkeresztúr located north to Mount Kopasz of Tokaj 513 m a.s.1. in Northeast Hungary. This area is the southwestern edge of Tokaj Mountains where the Great Plain of Hungary begins and river Bodrog flows into Tisza.

Two excavations were carried out at the site, in 1963 and 1982, and both found most of the artifacts in the top soil and the sediment underlying that was disturbed by agriculture (VÉRTES 1966; DOBOSI 2000b). Finds in undisturbed stratigraphic context lay on the border of a typical loess and an underlying limy, whitish loess like layer (DoBosi 2000b). A paleosol also was noticed at the site, which, according to VÉRTES (1966), lay under the cultural layer. Contrary, DoBOsI (2000b) associated the human occupation with the paleosol. The longest stratigraphic sequence of the site was located in unit I/8 of the 1982 excavation, where under the recent top soil a layer of loess, an embryonic soil, another layer of loess, an embryonic soil again, and the bedrock of "andesite tuff" composed the $2.50 \mathrm{~m}$ stratum from top to bottom (DoBOsi 2000b). A geological investigation (SüMEGi et al. 2000) found the strata consisted of 1) the agriculturally disturbed recent soil, 2) a reddish brown (dark brown when wet) paleosol of $10 \mathrm{~cm}$ thick woven by roots of recent vegetation and containing bones, lithic chips and charcoals in, 3) a yellowish brown calcareous loess layer with carbonate concretions $30 \mathrm{~cm}$ thick, 4) a $10 \mathrm{~cm}$ thick regolith layer of pebble and weathered fragments of rhyolite $1 \mathrm{~m}$ below the surface, and 5) the "rhyolite bedrock". These descriptions shows that the level of the archaeological finds was not related consistently with a single geological unit, and there are contradictions in the definitions of the geological units.

VÉRTES (1966) published charcoal samples ${ }^{14} \mathrm{C}$ dated to $28700 \pm 3000$ (GXO-195). VÉRTES recorded in the excavation diary that the sample had been taken from trench F, $10-15 \mathrm{~cm}$ thick amorphous patches of charcoals in two levels $(100-110 \mathrm{~cm}$ and 140-150 cm beneath the top soil), within which artifacts were not found. Vértes did not specify which level yielded the samples for dating. The second radiocarbon date of the site was obtained from samples taken during the geological investigation (SüMEGI et al. 2000). The samples (pine tree charcoals) were derived from a $5 \mathrm{~kg}$ sample of the paleosol taken for malacology and the charcoals found within sample were dated to $26318 \pm 365$ (Deb-2555). Two further dates on bone samples were obtained, $18575 \pm 208$ (Deb-3381) and $10630 \pm 270$ (Hv-12986) (Dobosi 2000b).

Dobosi (2000) classified the lithic assemblage Pavlovian, but the latest typological analysis pointed out a cultural affiliation with the Late Gravettian (LENGYEL 2015, 2016).

The archaeological material, besides the lithic tools, contains faunal remains (VöRös 2000) and an incised flat pebble interpreted as a lunar calendar (VÉRTES 1965). According to VöRÖs (2000), the most frequent hunted animals were the horse $(\mathrm{MNI}=50)$ and the elk $(\mathrm{MNI}=34)$. Further prey remains are deer, bison, mammoth, lion and hare. 
Knapped lithic analysis here contains material from the two excavation campaigns, including finds from the surface of the trenches, from the disturbed matrix and the undisturbed archaeological layer. Lithic artifacts collected by other field surveys were excluded. In spite of the poor preservation of archaeological features, the lithic assemblage is handled as a whole. Separating finds of the surface from those found buried was unnecessary because the agriculture distorted the most of the original settlement features. This decision most likely has not resulted in studying an admixture of finds from different periods of the Upper Palaeolithic because the only diagnostic tools are of Late Gravettian origin.

\section{Raw materials}

Local raw materials dominate the assemblage (Tab. 28). The most frequent kind among these are the metasomatically silicified rhyolite tuff and the limnic silicite. Further locally available materials are the obsidian of Carpathian 2 type and the red jasper. The local materials yielded 64.9 items per $\mathrm{kg}$.

Regional materials are the Carpathian 1 type obsidian, the radiolarite, the black Ondava chert and menilite of East Slovakia. The only regional material from west is the meta-rhyolite of Bükk mountains. Regional materials yielded 146.4 items per kg.

Transcarpathian materials are mostly the Cretaceous flints of Volhynian and Silesian origin, and in smaller portion the Jurassic flint from the Kraków-Częstochowa upland. Transcarpathian materials yielded 199 items per $\mathrm{kg}$.

\section{Blade tool production}

Materials of each procurement zone were processed with a full debitage cycle at the site (Tab. 29). By technological categories, flakes dominate the assemblage, but counting together the blades, neo-crested blades and crested blades, the number of blades becomes only slightly lower than that of the flakes. The predominance of the blade technology is also supported by that blade cores almost three times outnumber flake cores.

The scar pattern on the blades (Tab. 30) and the number of striking platforms of the cores (Tab. 31) identify unipolar blade technology, which kept the striking platforms plain (Tab. 32), and applied soft hammer technique (Tab. 33).

Local blades are longer, wider and thicker than the others (Tab. 34). ANOVA test (Tab. 35), however, found these differences significant only between local and transcarpathian specimens. Regional blades are only wider than local ones, and generally those do not differ from transcarpathian blades.

Overall, blades were the prime blanks of tools (Tab. 36). Blade usage ratio for tools is $19.2 \%$ opposing the $11.2 \%$ for the flakes (Tab. 37). Most of the blade tools were made of local and TC materials.

The length of local blade tools is shorter than that of the blank blades, but the thicknesses and the widths have the same mean value (Tabs 38, 39). Regional blades do not show difference in this comparison. Tool blades of TC are thicker and wider than the blank specimens. Comparing the sizes of the blade tools by raw materials, ANOVA (Tab. 40) found no difference between the raw materials. 
The blades are mostly edge retouched tools, burins, end-scrapers, and armatures (Tabs 41-43). Among regional blade tools edge retouched items are highly dominant and the majority of the transcarpathian blade tools are burins. Armatures mostly are local and transcarpathian blades. Between these two materials there is no significant difference in the shares of the types. The armature class typically is composed of points and backed bladelets (Tab. 42). The points are tip retouched and Gravette/ microgravette types (Tab. 43).

\section{Flake tool production}

The number of flake cores showed that part of the flakes were produced by a flake debitage (Tab. 39). Flake debitage was applied mostly to local and regional materials reduction. It is uncertain to separate the blade production by-product flakes and flakes made by their own debitage.

The flake cores typically have multiple debitage surfaces or a single striking platform (Tab. 44). Unidirectional scars dominate the dorsal faces of the flakes in each raw material group (Tab. 45), but the multiple direction is the most frequent on local raw material flakes in accordance with the multi-platform core frequency. Platforms of the flakes are plain (Tab. 46). A greater difference was found concerning the use of knapping technique in the flake production: local flakes bear more impact point and unabraded overhang (Tab. 47), signaling more frequent hard hammer technique.

The largest flakes are in the local material assemblage concerning all three measured parameters (Tab. 48). The mean length, width and thickness by raw materials are different (Tab. 49). The post hoc test showed that the mean lengths and widths of local flakes are greater compared to regional and transcarpathian flakes. Regarding thickness, local flakes have greater mean value than transcarpathian flakes.

Local flake tools (Tab. 50) are longer and thicker than flake blanks (Tab. 51). Transcarpathian flake tools are greater by all three measured parameter than blank flakes while regional flake tool and blank mean values are not different. Also tools of local material are greater in length, width and thickness than those in the other two groups (Tab. 52).

Most of the flake tools are end-scrapers, burins and retouched items (Tab. 53). There is no raw material which would have yielded all the flake tool types. Local and regional flakes tend to be end-scrapers while the transcarpathians are rather burins.

There are 12 tools made of debris, mostly burins, and except one splintered tool these were made of local raw material (Tab. 57). Rejuvenating flakes were also used from each raw material group for making end-scraper, burin, edge retouched and a borer. 
Table 28. Bodrogkeresztúr lithic raw material composition by weight in grams

\begin{tabular}{|l|c|c|c|c|c|c|c|}
\hline Raw material & Blade & Flake & Debris & Core & Raw block & Total & $\%$ \\
\hline Local & 3878 & 14945 & 4623 & 11287 & 116 & 34849 & 88.98223 \\
\hline Within local \% & 11.12801 & 42.88502 & 13.2658 & 32.3883 & 0.332865 & 100 & \\
\hline Regional & 562 & 882 & 147 & 985 & & 2576 & 6.577469 \\
\hline Within regional \% & 21.81677 & 34.23913 & 5.706522 & 38.23758 & 0 & 100 & \\
\hline Transcarpathian & 1184 & 444 & 86 & 25 & & 1739 & 4.440302 \\
\hline Within transcarpathian \% & 68.08511 & 25.53191 & 4.945371 & 1.437608 & 0 & 100 & \\
\hline Total & 5624 & 16271 & 4856 & 12297 & 116 & 39164 & 100 \\
\hline Within total \% & 14.36013 & 41.54581 & 12.39914 & 31.39873 & 0.29619 & 100 & \\
\hline
\end{tabular}

Table 29. Bodrogkeresztúr lithic assemblage composition by raw material types and technological categories

\begin{tabular}{|c|c|c|c|c|c|}
\hline & \multicolumn{3}{|c|}{ Raw material } & \multirow{2}{*}{ Total } \\
\hline & & local & regional & transcarpathian & \\
\hline \multirow[t]{2}{*}{ Flake } & count & 1089 & 186 & 102 & 1377 \\
\hline & $\%$ within raw material & $48.3 \%$ & $49.3 \%$ & $29.5 \%$ & $46.3 \%$ \\
\hline \multirow{2}{*}{ Blade } & count & 657 & 140 & 204 & 1001 \\
\hline & $\%$ within raw material & $29.2 \%$ & $37.1 \%$ & $59.0 \%$ & $33.6 \%$ \\
\hline \multirow[t]{2}{*}{ Debris } & count & 212 & 22 & 14 & 248 \\
\hline & $\%$ within raw material & $9.4 \%$ & $5.8 \%$ & $4.0 \%$ & $8.3 \%$ \\
\hline \multirow{2}{*}{$\begin{array}{l}\text { Rejuvenating } \\
\text { flake }\end{array}$} & count & 46 & 4 & 4 & 54 \\
\hline & $\%$ within raw material & $2.0 \%$ & $1.1 \%$ & $1.2 \%$ & $1.8 \%$ \\
\hline \multirow[t]{2}{*}{ Crest } & count & 21 & 0 & 3 & 24 \\
\hline & $\%$ within raw material & $0.9 \%$ & $0.0 \%$ & $0.9 \%$ & $0.8 \%$ \\
\hline \multirow[t]{2}{*}{ Neo-crest } & count & 66 & 5 & 14 & 85 \\
\hline & \% within raw material & $2.9 \%$ & $1.3 \%$ & $4.0 \%$ & $2.9 \%$ \\
\hline \multirow[t]{2}{*}{ Blade core } & count & 118 & 11 & 5 & 134 \\
\hline & \% within raw material & $5.2 \%$ & $2.9 \%$ & $1.4 \%$ & $4.5 \%$ \\
\hline \multirow[t]{2}{*}{ Flake core } & count & 44 & 9 & 0 & 53 \\
\hline & $\%$ within raw material & $2.0 \%$ & $2.4 \%$ & $0.0 \%$ & $1.8 \%$ \\
\hline \multirow{2}{*}{ Total } & count & 2253 & 377 & 346 & 2976 \\
\hline & $\%$ within raw material & $100.0 \%$ & $100.0 \%$ & $100.0 \%$ & $100.0 \%$ \\
\hline
\end{tabular}

Table 30. Bodrogkeresztúr blade assemblage (complete specimens) dorsal scar pattern frequency by raw material

\begin{tabular}{|c|c|c|c|c|c|c|}
\hline & \multicolumn{3}{|c|}{ Raw material } & \multirow{2}{*}{ Total } \\
\hline & & & local & regional & transcarpathian & \\
\hline \multirow[t]{10}{*}{ Scars } & \multirow[t]{2}{*}{ unidirectional } & count & 110 & 10 & 19 & 139 \\
\hline & & \% within raw material & $58.8 \%$ & $43.5 \%$ & $67.9 \%$ & $58.4 \%$ \\
\hline & \multirow[t]{2}{*}{ opposite } & count & 23 & 5 & 4 & 32 \\
\hline & & $\%$ within raw material & $12.3 \%$ & $21.7 \%$ & $14.3 \%$ & $13.4 \%$ \\
\hline & \multirow[t]{2}{*}{ perpendicular } & count & 41 & 5 & 5 & 51 \\
\hline & & $\%$ within raw material & $21.9 \%$ & $21.7 \%$ & $17.9 \%$ & $21.4 \%$ \\
\hline & \multirow[t]{2}{*}{ multiple } & count & 9 & 3 & 0 & 12 \\
\hline & & $\%$ within raw material & $4.8 \%$ & $13.0 \%$ & $0.0 \%$ & $5.0 \%$ \\
\hline & \multirow[t]{2}{*}{ no scar } & count & 4 & 0 & 0 & 4 \\
\hline & & $\%$ within raw material & $2.1 \%$ & $0.0 \%$ & $0.0 \%$ & $1.7 \%$ \\
\hline \multirow{2}{*}{\multicolumn{2}{|c|}{ Total }} & count & 187 & 23 & 28 & 238 \\
\hline & & $\%$ within raw material & $100.0 \%$ & $100.0 \%$ & $100.0 \%$ & $100.0 \%$ \\
\hline
\end{tabular}


Table 31. Bodrogkeresztúr blade core types by raw material

\begin{tabular}{|c|c|c|c|c|c|c|}
\hline & \multicolumn{3}{|c|}{ Raw material } & \multirow{2}{*}{ Total } \\
\hline & & & local & regional & transcarpathian & \\
\hline \multirow[t]{12}{*}{ Types } & \multirow[t]{2}{*}{ unidirectional } & count & 89 & 9 & 3 & 101 \\
\hline & & $\%$ within raw material & $75.4 \%$ & $81.8 \%$ & $60.0 \%$ & $75.4 \%$ \\
\hline & \multirow[t]{2}{*}{ bidirectional } & count & 10 & 0 & 0 & 10 \\
\hline & & $\%$ within raw material & $8.5 \%$ & $0.0 \%$ & $0.0 \%$ & $7.5 \%$ \\
\hline & \multirow[t]{2}{*}{ alternate } & count & 11 & 0 & 1 & 12 \\
\hline & & $\%$ within raw material & $9.3 \%$ & $0.0 \%$ & $20.0 \%$ & $9.0 \%$ \\
\hline & \multirow[t]{2}{*}{ multidirectional } & count & 1 & 0 & 1 & 2 \\
\hline & & $\%$ within raw material & $0.8 \%$ & $0.0 \%$ & $20.0 \%$ & $1.5 \%$ \\
\hline & \multirow[t]{2}{*}{ pre-core } & count & 1 & 0 & 0 & 1 \\
\hline & & $\%$ within raw material & $0.8 \%$ & $0.0 \%$ & $0.0 \%$ & $0.7 \%$ \\
\hline & \multirow[t]{2}{*}{ core fragment } & count & 6 & 2 & 0 & 8 \\
\hline & & $\%$ within raw material & $5.1 \%$ & $18.2 \%$ & $0.0 \%$ & $6.0 \%$ \\
\hline \multirow{2}{*}{\multicolumn{2}{|c|}{ Total }} & count & 118 & 11 & 5 & 134 \\
\hline & & $\%$ within raw material & $100.0 \%$ & $100.0 \%$ & $100.0 \%$ & $100.0 \%$ \\
\hline
\end{tabular}

Table 32. Bodrogkeresztúr blade assemblage (complete specimens) platform type frequency by raw material

\begin{tabular}{|c|c|c|c|c|c|c|}
\hline & \multicolumn{3}{|c|}{ Raw material } & \multirow{2}{*}{ Total } \\
\hline & & & local & regional & transcarpathian & \\
\hline \multirow[t]{14}{*}{ Platform } & \multirow[t]{2}{*}{ plain } & count & 132 & 13 & 17 & 162 \\
\hline & & $\%$ within raw material & $70.6 \%$ & $56.5 \%$ & $60.7 \%$ & $68.1 \%$ \\
\hline & \multirow[t]{2}{*}{ dihedral } & count & 14 & 1 & 3 & 18 \\
\hline & & $\%$ within raw material & $7.5 \%$ & $4.3 \%$ & $10.7 \%$ & $7.6 \%$ \\
\hline & \multirow[t]{2}{*}{ faceted } & count & 17 & 4 & 6 & 27 \\
\hline & & $\%$ within raw material & $9.1 \%$ & $17.4 \%$ & $21.4 \%$ & $11.3 \%$ \\
\hline & \multirow{2}{*}{ cortical } & count & 6 & 0 & 0 & 6 \\
\hline & & $\%$ within raw material & $3.2 \%$ & $0.0 \%$ & $0.0 \%$ & $2.5 \%$ \\
\hline & \multirow[t]{2}{*}{ linear } & count & 12 & 1 & 1 & 14 \\
\hline & & $\%$ within raw material & $6.4 \%$ & $4.3 \%$ & $3.6 \%$ & $5.9 \%$ \\
\hline & \multirow[t]{2}{*}{ punctiform } & count & 4 & 3 & 1 & 8 \\
\hline & & $\%$ within raw material & $2.1 \%$ & $13.0 \%$ & $3.6 \%$ & $3.4 \%$ \\
\hline & \multirow[t]{2}{*}{ irregular } & count & 2 & 1 & 0 & 3 \\
\hline & & $\%$ within raw material & $1.1 \%$ & $4.3 \%$ & $0.0 \%$ & $1.3 \%$ \\
\hline \multirow{2}{*}{\multicolumn{2}{|c|}{ Total }} & count & 187 & 23 & 28 & 238 \\
\hline & & $\%$ within raw material & $100.0 \%$ & $100.0 \%$ & $100.0 \%$ & $100.0 \%$ \\
\hline
\end{tabular}


Table 33. Bodrogkeresztúr blade assemblage (complete specimens) impact point-overhang co-presence by raw material

\begin{tabular}{|c|c|c|c|c|c|c|}
\hline & \multicolumn{3}{|c|}{ Raw material } & \multirow{2}{*}{ Total } \\
\hline & & & local & regional & transcarpathian & \\
\hline \multirow{8}{*}{$\begin{array}{l}\text { Impact } \\
\text { point-overhang }\end{array}$} & \multirow[t]{2}{*}{ none } & count & 119 & 15 & 26 & 160 \\
\hline & & $\%$ within raw material & $63.6 \%$ & $65.2 \%$ & $92.9 \%$ & $67.2 \%$ \\
\hline & \multirow[t]{2}{*}{ yes-no } & count & 16 & 3 & 0 & 19 \\
\hline & & $\%$ within raw material & $8.6 \%$ & $13.0 \%$ & $0.0 \%$ & $8.0 \%$ \\
\hline & \multirow[t]{2}{*}{ yes-yes } & count & 20 & 2 & 0 & 22 \\
\hline & & $\%$ within raw material & $10.7 \%$ & $8.7 \%$ & $0.0 \%$ & $9.2 \%$ \\
\hline & \multirow[t]{2}{*}{ no-yes } & count & 32 & 3 & 2 & 37 \\
\hline & & $\%$ within raw material & $17.1 \%$ & $13.0 \%$ & $7.1 \%$ & $15.5 \%$ \\
\hline \multirow{2}{*}{\multicolumn{2}{|c|}{ Total }} & count & 187 & 23 & 28 & 238 \\
\hline & & $\%$ within raw material & $100.0 \%$ & $100.0 \%$ & $100.0 \%$ & $100.0 \%$ \\
\hline
\end{tabular}

Table 34. Bodrogkeresztúr blade assemblage (complete specimens) length, width and thickness by raw material

\begin{tabular}{|c|c|c|c|c|}
\hline \multicolumn{2}{|l|}{ Raw material } & Length $[\mathrm{mm}]$ & Width [mm] & Thickness [mm] \\
\hline \multirow[t]{6}{*}{ Local } & Minimum & 6.10 & 6.30 & 1.70 \\
\hline & Maximum & 125.90 & 50.00 & 28.50 \\
\hline & Mean & 50.5225 & 19.0535 & 7.0856 \\
\hline & Median & 47.2000 & 17.6000 & 6.2000 \\
\hline & $\mathrm{N}$ & 187 & 187 & 187 \\
\hline & Std. deviation & 18.19229 & 6.80854 & 3.66159 \\
\hline \multirow[t]{6}{*}{ Regional } & Minimum & 18.20 & 5.50 & 1.50 \\
\hline & Maximum & 77.20 & 33.00 & 14.00 \\
\hline & Mean & 42.4043 & 15.2957 & 5.7957 \\
\hline & Median & 39.0000 & 13.0000 & 5.1000 \\
\hline & $\mathrm{N}$ & 23 & 23 & 23 \\
\hline & Std. deviation & 16.44977 & 7.03753 & 3.07918 \\
\hline \multirow[t]{6}{*}{ Transcarpathian } & Minimum & 19.50 & 6.20 & 1.70 \\
\hline & Maximum & 89.10 & 31.00 & 9.90 \\
\hline & Mean & 40.2857 & 12.6500 & 4.1321 \\
\hline & Median & 37.0000 & 9.8000 & 3.6000 \\
\hline & $\mathrm{N}$ & 28 & 28 & 28 \\
\hline & Std. deviation & 18.09112 & 6.94574 & 1.90808 \\
\hline \multirow[t]{6}{*}{ Total } & Minimum & 6.10 & 5.50 & 1.50 \\
\hline & Maximum & 125.90 & 50.00 & 28.50 \\
\hline & Mean & 48.5336 & 17.9370 & 6.6134 \\
\hline & Median & 45.5000 & 16.8000 & 5.7000 \\
\hline & $\mathrm{N}$ & 238 & 238 & 238 \\
\hline & Std. deviation & 18.35616 & 7.17198 & 3.57564 \\
\hline
\end{tabular}


Table 35. Bodrogkeresztúr blade assemblage (complete specimens) ANOVA and Tukey post hoc test to compare length, width and thickness by raw materials

ANOVA

\begin{tabular}{|l|l|r|r|r|c|c|}
\hline \multicolumn{2}{|c|}{} & Sum of squares & df & Mean square & F & Sig. \\
\hline \multirow{3}{*}{ Length $[\mathrm{mm}]$} & between groups & 3508.522 & 2 & 1754.261 & 5.400 & 0.005 \\
\cline { 2 - 7 } & within groups & 76348.310 & 235 & 324.886 & & \\
\cline { 2 - 7 } & Total & 79856.831 & 237 & & & \\
\hline \multirow{3}{*}{ Width $[\mathrm{mm}]$} & between groups & 1176.230 & 2 & 588.115 & 12.548 & 0.000 \\
\cline { 2 - 7 } & within groups & 11014.425 & 235 & 46.870 & & \\
\cline { 2 - 7 } & Total & 12190.655 & 237 & & & \\
\hline \multirow{2}{*}{ Thickness [mm] } & between groups & 229.455 & 2 & 114.728 & 9.627 & 0.000 \\
\cline { 2 - 7 } & within groups & 2800.642 & 235 & 11.918 & & \\
\cline { 2 - 7 } & Total & 3030.097 & 237 & & & \\
\hline
\end{tabular}

Multiple Comparisons

Tukey HSD

\begin{tabular}{|c|c|c|c|c|c|c|c|}
\hline \multirow{2}{*}{$\begin{array}{l}\text { Dependent } \\
\text { variable }\end{array}$} & \multirow{2}{*}{ (I) raw material } & \multirow{2}{*}{ (J) raw material } & \multirow{2}{*}{$\begin{array}{c}\text { Mean } \\
\text { difference } \\
(\mathrm{I}-\mathrm{J})\end{array}$} & \multirow{2}{*}{$\begin{array}{l}\text { Std. } \\
\text { error }\end{array}$} & \multirow{2}{*}{ Sig. } & \multicolumn{2}{|c|}{$\begin{array}{c}\text { 95\% Confidence } \\
\text { interval }\end{array}$} \\
\hline & & & & & & $\begin{array}{l}\text { Lower } \\
\text { bound }\end{array}$ & $\begin{array}{l}\text { Upper } \\
\text { bound }\end{array}$ \\
\hline \multirow{6}{*}{$\begin{array}{l}\text { Length } \\
{[\mathrm{mm}]}\end{array}$} & \multirow[t]{2}{*}{ local } & regional & 8.11811 & 3.98282 & 0.105 & -1.2760 & 17.5122 \\
\hline & & transcarpathian & 10.23675 & 3.65246 & 0.015 & 1.6218 & 18.8517 \\
\hline & \multirow[t]{2}{*}{ regional } & local & -8.11811 & 3.98282 & 0.105 & -17.5122 & 1.2760 \\
\hline & & transcarpathian & 2.11863 & 5.07234 & 0.908 & -9.8453 & 14.0826 \\
\hline & \multirow[t]{2}{*}{ transcarpathian } & local & -10.23675 & 3.65246 & 0.015 & -18.8517 & -1.6218 \\
\hline & & regional & -2.11863 & 5.07234 & 0.908 & -14.0826 & 9.8453 \\
\hline \multirow{6}{*}{$\begin{array}{l}\text { Width } \\
{[\mathrm{mm}]}\end{array}$} & \multirow[t]{2}{*}{ local } & regional & 3.75782 & 1.51277 & 0.036 & 0.1897 & 7.3259 \\
\hline & & transcarpathian & 6.40348 & 1.38729 & 0.000 & 3.1313 & 9.6756 \\
\hline & \multirow[t]{2}{*}{ regional } & local & -3.75782 & 1.51277 & 0.036 & -7.3259 & -0.1897 \\
\hline & & transcarpathian & 2.64565 & 1.92659 & 0.357 & -1.8985 & 7.1898 \\
\hline & \multirow[t]{2}{*}{ transcarpathian } & local & -6.40348 & 1.38729 & 0.000 & -9.6756 & -3.1313 \\
\hline & & regional & -2.64565 & 1.92659 & 0.357 & -7.1898 & 1.8985 \\
\hline \multirow{6}{*}{$\begin{array}{l}\text { Thickness } \\
{[\mathrm{mm}]}\end{array}$} & \multirow[t]{2}{*}{ local } & regional & 1.28991 & 0.76282 & 0.211 & -0.5093 & 3.0891 \\
\hline & & transcarpathian & 2.95342 & 0.69954 & 0.000 & 1.3034 & 4.6034 \\
\hline & \multirow[t]{2}{*}{ regional } & local & -1.28991 & 0.76282 & 0.211 & -3.0891 & 0.5093 \\
\hline & & transcarpathian & 1.66351 & 0.97149 & 0.203 & -0.6279 & 3.9549 \\
\hline & \multirow[t]{2}{*}{ transcarpathian } & local & -2.95342 & 0.69954 & 0.000 & -4.6034 & -1.3034 \\
\hline & & regional & -1.66351 & 0.97149 & 0.203 & -3.9549 & 0.6279 \\
\hline
\end{tabular}


Lithic analysis of the Middle and Late Upper Palaeolithic in Hungary

Table 36. Bodrogkeresztúr tool assemblage product composition by raw materials

\begin{tabular}{|c|c|c|c|c|c|}
\hline & \multicolumn{3}{|c|}{ Raw material } & \multirow{2}{*}{ Total } \\
\hline & & local & regional & transcarpathian & \\
\hline \multirow[t]{2}{*}{ Flake } & count & 91 & 40 & 23 & 154 \\
\hline & $\%$ within raw material & $47.2 \%$ & $50.0 \%$ & $20.7 \%$ & $40.1 \%$ \\
\hline \multirow[t]{2}{*}{ Blade } & count & 89 & 38 & 86 & 213 \\
\hline & $\%$ within raw material & $46.1 \%$ & $47.5 \%$ & $77.5 \%$ & $55.5 \%$ \\
\hline \multirow[t]{2}{*}{ Debris } & count & 11 & 1 & 0 & 12 \\
\hline & $\%$ within raw material & $5.7 \%$ & $1.3 \%$ & $0.0 \%$ & $3.1 \%$ \\
\hline \multirow{2}{*}{$\begin{array}{l}\text { Rejuvenating } \\
\text { flake }\end{array}$} & count & 2 & 1 & 2 & 5 \\
\hline & $\%$ within raw material & $1.0 \%$ & $1.3 \%$ & $1.8 \%$ & $1.3 \%$ \\
\hline \multirow{2}{*}{ Total } & count & 193 & 80 & 111 & 384 \\
\hline & $\%$ within raw material & $100.0 \%$ & $100.0 \%$ & $100.0 \%$ & $100.0 \%$ \\
\hline
\end{tabular}

Table 37. Bodrogkeresztúr knapped product frequency in tool assemblage by raw materials

\begin{tabular}{|c|c|c|c|c|c|c|c|}
\hline & \multicolumn{3}{|c|}{ Raw material } & \multirow{2}{*}{ Total } \\
\hline & & & & local & regional & transcarpathian & \\
\hline \multirow[t]{6}{*}{ Flake } & \multirow[t]{4}{*}{ state } & \multirow[t]{2}{*}{ blank } & count & 998 & 146 & 79 & 1223 \\
\hline & & & $\%$ within raw material & $91.6 \%$ & $78.5 \%$ & $77.5 \%$ & $88.8 \%$ \\
\hline & & \multirow[t]{2}{*}{ tool } & count & 91 & 40 & 23 & 154 \\
\hline & & & $\%$ within raw material & $8.4 \%$ & $21.5 \%$ & $22.5 \%$ & $11.2 \%$ \\
\hline & \multirow{2}{*}{\multicolumn{2}{|c|}{ total }} & count & 1089 & 186 & 102 & 1377 \\
\hline & & & $\%$ within raw material & $100.0 \%$ & $100.0 \%$ & $100.0 \%$ & $100.0 \%$ \\
\hline \multirow[t]{6}{*}{ Blade } & \multirow[t]{4}{*}{ state } & \multirow[t]{2}{*}{ blank } & count & 655 & 107 & 135 & 897 \\
\hline & & & $\%$ within raw material & $88.0 \%$ & $73.8 \%$ & $61.1 \%$ & $80.8 \%$ \\
\hline & & \multirow[t]{2}{*}{ tool } & count & 89 & 38 & 86 & 213 \\
\hline & & & $\%$ within raw material & $12.0 \%$ & $26.2 \%$ & $38.9 \%$ & $19.2 \%$ \\
\hline & \multirow{2}{*}{\multicolumn{2}{|c|}{ total }} & count & 744 & 145 & 221 & 1110 \\
\hline & & & $\%$ within raw material & $100.0 \%$ & $100.0 \%$ & $100.0 \%$ & $100.0 \%$ \\
\hline \multirow[t]{6}{*}{ Debris } & \multirow[t]{4}{*}{ state } & \multirow[t]{2}{*}{ blank } & count & 201 & 21 & 14 & 236 \\
\hline & & & $\%$ within raw material & $94.8 \%$ & $95.5 \%$ & $100.0 \%$ & $95.2 \%$ \\
\hline & & \multirow[t]{2}{*}{ tool } & count & 11 & 1 & 0 & 12 \\
\hline & & & $\%$ within raw material & $5.2 \%$ & $4.5 \%$ & $0.0 \%$ & $4.8 \%$ \\
\hline & \multirow{2}{*}{\multicolumn{2}{|c|}{ total }} & count & 212 & 22 & 14 & 248 \\
\hline & & & $\%$ within raw material & $100.0 \%$ & $100.0 \%$ & $100.0 \%$ & $100.0 \%$ \\
\hline \multirow{6}{*}{$\begin{array}{l}\text { Rejuvenating } \\
\text { flake }\end{array}$} & \multirow[t]{4}{*}{ state } & \multirow[t]{2}{*}{ blank } & count & 44 & 3 & 2 & 49 \\
\hline & & & $\%$ within raw material & $95.7 \%$ & $75.0 \%$ & $50.0 \%$ & $90.7 \%$ \\
\hline & & \multirow[t]{2}{*}{ tool } & count & 2 & 1 & 2 & 5 \\
\hline & & & $\%$ within raw material & $4.3 \%$ & $25.0 \%$ & $50.0 \%$ & $9.3 \%$ \\
\hline & \multirow{2}{*}{\multicolumn{2}{|c|}{ total }} & count & 46 & 4 & 4 & 54 \\
\hline & & & $\%$ within raw material & $100.0 \%$ & $100.0 \%$ & $100.0 \%$ & $100.0 \%$ \\
\hline
\end{tabular}


Table 38. Bodrogkeresztúr blade tool assemblage length, width and thickness by raw materials

\begin{tabular}{|c|c|c|c|c|}
\hline \multicolumn{2}{|l|}{ Raw material } & Length [mm] & Width [mm] & Thickness [mm] \\
\hline \multirow[t]{6}{*}{ Local } & Minimum & 10.10 & 5.10 & 1.70 \\
\hline & Maximum & 107.90 & 44.30 & 18.80 \\
\hline & Mean & 42.3674 & 19.8798 & 7.7146 \\
\hline & Median & 34.6000 & 19.5000 & 7.1000 \\
\hline & $\mathrm{N}$ & 89 & 89 & 89 \\
\hline & Std. deviation & 22.97158 & 9.09991 & 4.19533 \\
\hline \multirow[t]{6}{*}{ Regional } & Minimum & 12.50 & 6.80 & 2.50 \\
\hline & Maximum & 123.50 & 33.80 & 12.80 \\
\hline & Mean & 42.5921 & 18.5474 & 6.6632 \\
\hline & Median & 40.4000 & 17.4500 & 6.2500 \\
\hline & $\mathrm{N}$ & 38 & 38 & 38 \\
\hline & Std. deviation & 20.89442 & 6.94125 & 2.88035 \\
\hline \multirow[t]{6}{*}{ Transcarpathian } & Minimum & 14.00 & 4.10 & 2.00 \\
\hline & Maximum & 110.00 & 39.00 & 13.90 \\
\hline & Mean & 46.5965 & 19.9826 & 6.7674 \\
\hline & Median & 45.2000 & 20.4500 & 6.8000 \\
\hline & $\mathrm{N}$ & 86 & 86 & 86 \\
\hline & Std. deviation & 21.35264 & 7.90186 & 2.80044 \\
\hline \multirow[t]{6}{*}{ Total } & Minimum & 10.10 & 4.10 & 1.70 \\
\hline & Maximum & 123.50 & 44.30 & 18.80 \\
\hline & Mean & 44.1150 & 19.6836 & 7.1446 \\
\hline & Median & 39.0000 & 20.0000 & 6.9000 \\
\hline & $\mathrm{N}$ & 213 & 213 & 213 \\
\hline & Std. deviation & 21.95991 & 8.25232 & 3.48339 \\
\hline
\end{tabular}

Table 39. Bodrogkeresztúr blade blank and tool length, width and thickness mean t-test comparison by raw material

\begin{tabular}{|c|c|c|c|c|c|c|c|c|}
\hline \multicolumn{9}{|c|}{ Independent Samples Test } \\
\hline \multirow{2}{*}{\multicolumn{2}{|c|}{ Raw material }} & \multicolumn{2}{|c|}{$\begin{array}{c}\text { Levene's Test } \\
\text { for Equality } \\
\text { of Variances }\end{array}$} & \multicolumn{5}{|c|}{ t-test for Equality of Means } \\
\hline & & $\mathrm{F}$ & Sig. & $\mathrm{t}$ & df & $\begin{array}{c}\text { Sig. } \\
\text { (2-tailed) }\end{array}$ & $\begin{array}{c}\text { Mean } \\
\text { difference }\end{array}$ & $\begin{array}{l}\text { Std. error } \\
\text { difference }\end{array}$ \\
\hline \multirow[t]{6}{*}{ Local } & \multirow{2}{*}{$\begin{array}{l}\text { length } \\
{[\mathrm{mm}]}\end{array}$} & 7.949 & 0.005 & 3.190 & 274 & 0.002 & 8.15504 & 2.55662 \\
\hline & & & & 2.939 & 142.375 & 0.004 & 8.15504 & 2.77470 \\
\hline & \multirow{2}{*}{$\begin{array}{l}\text { width } \\
{[\mathrm{mm}]}\end{array}$} & 13.728 & 0.000 & -0.842 & 274 & 0.400 & -0.82630 & 0.98127 \\
\hline & & & & -0.761 & 136.552 & 0.448 & -0.82630 & 1.08551 \\
\hline & \multirow{2}{*}{$\begin{array}{l}\text { thickness } \\
{[\mathrm{mm}]}\end{array}$} & 4.284 & 0.039 & -1.272 & 274 & 0.205 & -0.62905 & 0.49465 \\
\hline & & & & -1.212 & 153.809 & 0.227 & -0.62905 & 0.51909 \\
\hline \multirow[t]{6}{*}{ Regional } & \multirow{2}{*}{$\begin{array}{l}\text { length } \\
{[\mathrm{mm}]}\end{array}$} & 0.934 & 0.338 & -0.037 & 59 & 0.971 & -0.18776 & 5.11379 \\
\hline & & & & -0.039 & 54.848 & 0.969 & -0.18776 & 4.82223 \\
\hline & \multirow{2}{*}{$\begin{array}{l}\text { width } \\
{[\mathrm{mm}]}\end{array}$} & 0.234 & 0.630 & -1.764 & 59 & 0.083 & -3.25172 & 1.84330 \\
\hline & & & & -1.758 & 46.044 & 0.085 & -3.25172 & 1.84966 \\
\hline & \multirow{2}{*}{$\begin{array}{l}\text { thickness } \\
{[\mathrm{mm}]}\end{array}$} & 0.020 & 0.888 & -1.111 & 59 & 0.271 & -0.86751 & 0.78095 \\
\hline & & & & -1.092 & 44.116 & 0.281 & -0.86751 & 0.79408 \\
\hline \multirow[t]{6}{*}{ Transcarpathian } & \multirow{2}{*}{$\begin{array}{l}\text { length } \\
{[\mathrm{mm}]}\end{array}$} & 0.886 & 0.349 & -1.407 & 112 & 0.162 & -6.31080 & 4.48517 \\
\hline & & & & -1.531 & 53.547 & 0.132 & -6.31080 & 4.12195 \\
\hline & \multirow{2}{*}{$\begin{array}{l}\text { width } \\
{[\mathrm{mm}]}\end{array}$} & 0.882 & 0.350 & -4.387 & 112 & 0.000 & -7.33256 & 1.67153 \\
\hline & & & & -4.686 & 51.637 & 0.000 & -7.33256 & 1.56493 \\
\hline & \multirow{2}{*}{$\begin{array}{l}\text { thickness } \\
{[\mathrm{mm}]}\end{array}$} & 4.894 & 0.029 & -4.635 & 112 & 0.000 & -2.63530 & 0.56862 \\
\hline & & & & -5.603 & 67.591 & 0.000 & -2.63530 & 0.47034 \\
\hline
\end{tabular}


Lithic analysis of the Middle and Late Upper Palaeolithic in Hungary

Table 40. Bodrogkeresztúr blade tool length, width and thickness mean comparison by raw materials with ANOVA

ANOVA

\begin{tabular}{|l|l|r|r|r|c|c|}
\hline \multicolumn{2}{|c|}{} & Sum of squares & df & Mean square & F & Sig. \\
\hline \multirow{3}{*}{ Length $[\mathrm{mm}]$} & between groups & 889.520 & 2 & 444.760 & 0.922 & 0.399 \\
\cline { 2 - 7 } & within groups & 101344.892 & 210 & 482.595 & & \\
\cline { 2 - 7 } & Total & 102234.412 & 212 & & & \\
\hline \multirow{3}{*}{ Width [mm] } & between groups & 60.170 & 2 & 30.085 & 0.439 & 0.645 \\
\cline { 2 - 7 } & within groups & 14377.182 & 210 & 68.463 & & \\
\cline { 2 - 7 } & Total & 14437.352 & 212 & & & \\
\hline \multirow{3}{*}{ Thickness [mm] } & between groups & 49.958 & 2 & 24.979 & 2.080 & 0.128 \\
\cline { 2 - 7 } & within groups & 2522.448 & 210 & 12.012 & & \\
\cline { 2 - 7 } & Total & 2572.406 & 212 & & & \\
\hline
\end{tabular}

Table 41. Bodrogkeresztúr blade tool types by raw material

\begin{tabular}{|c|c|c|c|c|c|c|}
\hline & \multicolumn{3}{|c|}{ Raw material } & \multirow{2}{*}{ Total } \\
\hline & & & local & regional & transcarpathian & \\
\hline \multirow[t]{18}{*}{ Tooltypes } & \multirow[t]{2}{*}{ endscraper } & count & 19 & 11 & 10 & 40 \\
\hline & & $\%$ within raw material & $21.3 \%$ & $28.9 \%$ & $11.6 \%$ & $18.8 \%$ \\
\hline & \multirow[t]{2}{*}{ burin } & count & 15 & 5 & 31 & 51 \\
\hline & & $\%$ within raw material & $16.9 \%$ & $13.2 \%$ & $36.0 \%$ & $23.9 \%$ \\
\hline & \multirow[t]{2}{*}{ retouched } & count & 26 & 17 & 28 & 71 \\
\hline & & $\%$ within raw material & $29.2 \%$ & $44.7 \%$ & $32.6 \%$ & $33.3 \%$ \\
\hline & \multirow[t]{2}{*}{ borer } & count & 0 & 0 & 1 & 1 \\
\hline & & $\%$ within raw material & $0.0 \%$ & $0.0 \%$ & $1.2 \%$ & $0.5 \%$ \\
\hline & \multirow[t]{2}{*}{ splintered piece } & count & 1 & 0 & 0 & 1 \\
\hline & & \% within raw material & $1.1 \%$ & $0.0 \%$ & $0.0 \%$ & $0.5 \%$ \\
\hline & \multirow[t]{2}{*}{ truncation } & count & 6 & 1 & 4 & 11 \\
\hline & & $\%$ within raw material & $6.7 \%$ & $2.6 \%$ & $4.7 \%$ & $5.2 \%$ \\
\hline & \multirow{2}{*}{\begin{tabular}{|l|} 
notched- \\
denticulated
\end{tabular}} & count & 5 & 0 & 0 & 5 \\
\hline & & $\%$ within raw material & $5.6 \%$ & $0.0 \%$ & $0.0 \%$ & $2.3 \%$ \\
\hline & \multirow[t]{2}{*}{ composite } & count & 1 & 1 & 1 & 3 \\
\hline & & $\%$ within raw material & $1.1 \%$ & $2.6 \%$ & $1.2 \%$ & $1.4 \%$ \\
\hline & \multirow[t]{2}{*}{ armature } & count & 16 & 3 & 11 & 30 \\
\hline & & \% within raw material & $18.0 \%$ & $7.9 \%$ & $12.8 \%$ & $14.1 \%$ \\
\hline \multirow{2}{*}{\multicolumn{2}{|c|}{ Total }} & count & 89 & 38 & 86 & 213 \\
\hline & & \% within raw material & $100.0 \%$ & $100.0 \%$ & $100.0 \%$ & $100.0 \%$ \\
\hline
\end{tabular}


Table 42. Bodrogkeresztúr armature types by raw material

\begin{tabular}{|c|c|c|c|c|c|c|}
\hline & \multicolumn{3}{|c|}{ Raw material } & \multirow{2}{*}{ Total } \\
\hline & & & local & regional & transcarpathian & \\
\hline \multirow[t]{10}{*}{ Armatures } & \multirow[t]{2}{*}{ backed } & count & 6 & 1 & 3 & 10 \\
\hline & & $\%$ within raw material & $37.5 \%$ & $33.3 \%$ & $27.3 \%$ & $33.3 \%$ \\
\hline & \multirow[t]{2}{*}{ backed-truncated } & count & 2 & 0 & 2 & 4 \\
\hline & & $\%$ within raw material & $12.5 \%$ & $0.0 \%$ & $18.2 \%$ & $13.3 \%$ \\
\hline & \multirow{2}{*}{$\begin{array}{l}\text { backed- } \\
\text { ventral truncation }\end{array}$} & count & 0 & 0 & 1 & 1 \\
\hline & & $\%$ within raw material & $0.0 \%$ & $0.0 \%$ & $9.1 \%$ & $3.3 \%$ \\
\hline & \multirow[t]{2}{*}{ trapeze-rectangle } & count & 0 & 0 & 1 & 1 \\
\hline & & $\%$ within raw material & $0.0 \%$ & $0.0 \%$ & $9.1 \%$ & $3.3 \%$ \\
\hline & \multirow[t]{2}{*}{ points } & count & 8 & 2 & 4 & 14 \\
\hline & & $\%$ within raw material & $50.0 \%$ & $66.7 \%$ & $36.4 \%$ & $46.7 \%$ \\
\hline \multirow{2}{*}{\multicolumn{2}{|c|}{ Total }} & count & 16 & 3 & 11 & 30 \\
\hline & & $\%$ within raw material & $100.0 \%$ & $100.0 \%$ & $100.0 \%$ & $100.0 \%$ \\
\hline
\end{tabular}

Table 43. Bodrogkeresztúr point types by raw material

\begin{tabular}{|c|c|c|c|c|c|c|}
\hline & \multicolumn{3}{|c|}{ Raw material } & \multirow{2}{*}{ Total } \\
\hline & & & local & regional & transcarpathian & \\
\hline \multirow[t]{10}{*}{ Points } & \multirow[t]{2}{*}{ retouched } & count & 3 & 0 & 1 & 4 \\
\hline & & $\%$ within raw material & $37.5 \%$ & $0.0 \%$ & $25.0 \%$ & $28.6 \%$ \\
\hline & \multirow{2}{*}{$\begin{array}{l}\text { gravette/ } \\
\text { microgravette }\end{array}$} & count & 3 & 1 & 1 & 5 \\
\hline & & $\%$ within raw material & $37.5 \%$ & $50.0 \%$ & $25.0 \%$ & $35.7 \%$ \\
\hline & \multirow[t]{2}{*}{ vachons } & count & 1 & 0 & 1 & 2 \\
\hline & & \% within raw material & $12.5 \%$ & $0.0 \%$ & $25.0 \%$ & $14.3 \%$ \\
\hline & \multirow[t]{2}{*}{ shouldered } & count & 0 & 1 & 0 & 1 \\
\hline & & $\%$ within raw material & $0.0 \%$ & $50.0 \%$ & $0.0 \%$ & $7.1 \%$ \\
\hline & \multirow[t]{2}{*}{ fléchette } & count & 1 & 0 & 1 & 2 \\
\hline & & $\%$ within raw material & $12.5 \%$ & $0.0 \%$ & $25.0 \%$ & $14.3 \%$ \\
\hline \multirow{2}{*}{\multicolumn{2}{|c|}{ Total }} & count & 8 & 2 & 4 & 14 \\
\hline & & $\%$ within raw material & $100.0 \%$ & $100.0 \%$ & $100.0 \%$ & $100.0 \%$ \\
\hline
\end{tabular}

Table 44. Bodrogkeresztúr flake core types by raw material

\begin{tabular}{|c|c|c|c|c|c|}
\hline & \multicolumn{2}{|c|}{ Raw material } & \multirow{2}{*}{ Total } \\
\hline & & & local & regional & \\
\hline \multirow[t]{10}{*}{ Types } & \multirow[t]{2}{*}{ unidirectional } & count & 15 & 2 & 17 \\
\hline & & $\%$ within raw material & $34.1 \%$ & $22.2 \%$ & $32.1 \%$ \\
\hline & \multirow[t]{2}{*}{ bidirctional } & count & 4 & 0 & 4 \\
\hline & & $\%$ within raw material & $9.1 \%$ & $0.0 \%$ & $7.5 \%$ \\
\hline & \multirow[t]{2}{*}{ alternate } & count & 1 & 0 & 1 \\
\hline & & $\%$ within raw material & $2.3 \%$ & $0.0 \%$ & $1.9 \%$ \\
\hline & \multirow[t]{2}{*}{ multidirectional } & count & 19 & 6 & 25 \\
\hline & & $\%$ within raw material & $43.2 \%$ & $66.7 \%$ & $47.2 \%$ \\
\hline & \multirow[t]{2}{*}{ core fragment } & count & 5 & 1 & 6 \\
\hline & & $\%$ within raw material & $11.4 \%$ & $11.1 \%$ & $11.3 \%$ \\
\hline \multirow{2}{*}{\multicolumn{2}{|c|}{ Total }} & count & 44 & 9 & 53 \\
\hline & & $\%$ within raw material & $100.0 \%$ & $100.0 \%$ & $100.0 \%$ \\
\hline
\end{tabular}


Table 45. Bodrogkeresztúr flake assemblage (complete specimens) dorsal scar pattern frequency by raw material

\begin{tabular}{|c|c|c|c|c|c|c|}
\hline & \multicolumn{3}{|c|}{ Raw material } & \multirow{2}{*}{ Total } \\
\hline & & & local & regional & transcarpathian & \\
\hline \multirow[t]{10}{*}{ Scars } & \multirow[t]{2}{*}{ unidirectional } & count & 165 & 22 & 14 & 201 \\
\hline & & $\%$ within raw material & $47.0 \%$ & $48.9 \%$ & $42.4 \%$ & $46.9 \%$ \\
\hline & \multirow[t]{2}{*}{ opposite } & count & 36 & 8 & 3 & 47 \\
\hline & & $\%$ within raw material & $10.3 \%$ & $17.8 \%$ & $9.1 \%$ & $11.0 \%$ \\
\hline & \multirow[t]{2}{*}{ perpendicular } & count & 90 & 11 & 15 & 116 \\
\hline & & $\%$ within raw material & $25.6 \%$ & $24.4 \%$ & $45.5 \%$ & $27.0 \%$ \\
\hline & \multirow[t]{2}{*}{ multiple } & count & 49 & 2 & 1 & 52 \\
\hline & & $\%$ within raw material & $14.0 \%$ & $4.4 \%$ & $3.0 \%$ & $12.1 \%$ \\
\hline & \multirow[t]{2}{*}{ no scar } & count & 11 & 2 & 0 & 13 \\
\hline & & \% within raw material & $3.1 \%$ & $4.4 \%$ & $0.0 \%$ & $3.0 \%$ \\
\hline \multirow{2}{*}{\multicolumn{2}{|c|}{ Total }} & count & 351 & 45 & 33 & 429 \\
\hline & & $\%$ within raw material & $100.0 \%$ & $100.0 \%$ & $100.0 \%$ & $100.0 \%$ \\
\hline
\end{tabular}

Table 46. Bodrogkeresztúr flake assemblage (complete specimens) platform type frequency by raw material

\begin{tabular}{|c|c|c|c|c|c|c|}
\hline & \multicolumn{3}{|c|}{ Raw material } & \multirow{2}{*}{ Total } \\
\hline & & & local & regional & transcarpathian & \\
\hline \multirow[t]{14}{*}{ Platform } & \multirow[t]{2}{*}{ plain } & count & 220 & 26 & 20 & 266 \\
\hline & & $\%$ within raw material & $62.7 \%$ & $57.8 \%$ & $60.6 \%$ & $62.0 \%$ \\
\hline & \multirow[t]{2}{*}{ dihedral } & count & 47 & 4 & 4 & 55 \\
\hline & & $\%$ within raw material & $13.4 \%$ & $8.9 \%$ & $12.1 \%$ & $12.8 \%$ \\
\hline & \multirow[t]{2}{*}{ faceted } & count & 37 & 4 & 6 & 47 \\
\hline & & $\%$ within raw material & $10.5 \%$ & $8.9 \%$ & $18.2 \%$ & $11.0 \%$ \\
\hline & \multirow[t]{2}{*}{ cortical } & count & 33 & 5 & 1 & 39 \\
\hline & & $\%$ within raw material & $9.4 \%$ & $11.1 \%$ & $3.0 \%$ & $9.1 \%$ \\
\hline & \multirow[t]{2}{*}{ linear } & count & 5 & 3 & 1 & 9 \\
\hline & & $\%$ within raw material & $1.4 \%$ & $6.7 \%$ & $3.0 \%$ & $2.1 \%$ \\
\hline & \multirow[t]{2}{*}{ punctiform } & count & 1 & 1 & 0 & 2 \\
\hline & & $\%$ within raw material & $0.3 \%$ & $2.2 \%$ & $0.0 \%$ & $0.5 \%$ \\
\hline & \multirow[t]{2}{*}{ irregular } & count & 8 & 2 & 1 & 11 \\
\hline & & $\%$ within raw material & $2.3 \%$ & $4.4 \%$ & $3.0 \%$ & $2.6 \%$ \\
\hline \multirow{2}{*}{\multicolumn{2}{|c|}{ Total }} & count & 351 & 45 & 33 & 429 \\
\hline & & $\%$ within raw material & $100.0 \%$ & $100.0 \%$ & $100.0 \%$ & $100.0 \%$ \\
\hline
\end{tabular}


Table 47. Bodrogkeresztúr flake assemblage (complete specimens) impact point-overhang presence frequency by raw material

\begin{tabular}{|c|c|c|c|c|c|c|}
\hline & \multicolumn{3}{|c|}{ Raw material } & \multirow{2}{*}{ Total } \\
\hline & & & local & regional & transcarpathian & \\
\hline \multirow{8}{*}{$\begin{array}{l}\text { Impact } \\
\text { point-overhang }\end{array}$} & \multirow[t]{2}{*}{ none } & count & 60 & 13 & 10 & 83 \\
\hline & & \% within raw material & $17.1 \%$ & $28.9 \%$ & $30.3 \%$ & $19.3 \%$ \\
\hline & \multirow[t]{2}{*}{ yes-no } & count & 52 & 7 & 7 & 66 \\
\hline & & $\%$ within raw material & $14.8 \%$ & $15.6 \%$ & $21.2 \%$ & $15.4 \%$ \\
\hline & \multirow[t]{2}{*}{ yes-yes } & count & 129 & 15 & 9 & 153 \\
\hline & & $\%$ within raw material & $36.8 \%$ & $33.3 \%$ & $27.3 \%$ & $35.7 \%$ \\
\hline & \multirow[t]{2}{*}{ no-yes } & count & 110 & 10 & 7 & 127 \\
\hline & & $\%$ within raw material & $31.3 \%$ & $22.2 \%$ & $21.2 \%$ & $29.6 \%$ \\
\hline \multirow{2}{*}{\multicolumn{2}{|c|}{ Total }} & count & 351 & 45 & 33 & 429 \\
\hline & & $\%$ within raw material & $100.0 \%$ & $100.0 \%$ & $100.0 \%$ & $100.0 \%$ \\
\hline
\end{tabular}

Table 48. Bodrogkeresztúr flake assemblage length, width and thickness by raw materials

\begin{tabular}{|c|c|c|c|c|}
\hline \multicolumn{2}{|l|}{ Raw material } & Length $[\mathrm{mm}]$ & Width [mm] & Thickness [mm] \\
\hline \multirow[t]{6}{*}{ Local } & Minimum & 9.00 & 3.70 & 1.40 \\
\hline & Maximum & 97.20 & 103.20 & 44.10 \\
\hline & Mean & 39.5276 & 33.4875 & 9.8385 \\
\hline & Median & 36.7000 & 31.3000 & 8.1000 \\
\hline & $\mathrm{N}$ & 351 & 351 & 351 \\
\hline & Std. deviation & 16.97545 & 15.41051 & 6.21462 \\
\hline \multirow[t]{6}{*}{ Regional } & Minimum & 9.70 & 10.50 & 2.10 \\
\hline & Maximum & 67.50 & 46.20 & 18.80 \\
\hline & Mean & 29.1489 & 24.2889 & 7.6800 \\
\hline & Median & 28.0000 & 23.8000 & 7.2000 \\
\hline & $\mathrm{N}$ & 45 & 45 & 45 \\
\hline & Std. deviation & 10.89668 & 8.08307 & 4.03837 \\
\hline \multirow[t]{6}{*}{ Transcarpathian } & Minimum & 13.20 & 7.30 & 1.90 \\
\hline & Maximum & 45.10 & 50.20 & 11.20 \\
\hline & Mean & 27.5182 & 23.1727 & 6.0303 \\
\hline & Median & 28.0000 & 22.1000 & 5.6000 \\
\hline & $\mathrm{N}$ & 33 & 33 & 33 \\
\hline & Std. deviation & 7.39368 & 8.39391 & 2.38124 \\
\hline \multirow[t]{6}{*}{ Total } & Minimum & 9.00 & 3.70 & 1.40 \\
\hline & Maximum & 97.20 & 103.20 & 44.10 \\
\hline & Mean & 37.5152 & 31.7291 & 9.3191 \\
\hline & Median & 33.8000 & 28.9000 & 7.9000 \\
\hline & $\mathrm{N}$ & 429 & 429 & 429 \\
\hline & Std. deviation & 16.44171 & 14.83877 & 5.91787 \\
\hline
\end{tabular}


Table 49. Bodrogkeresztúr flake length, width and thickness mean comparison by raw materials with ANOVA and the Tukey post hoc

ANOVA

\begin{tabular}{|l|l|r|r|r|r|c|}
\hline \multicolumn{2}{|c|}{} & Sum of squares & df & Mean square & F & Sig. \\
\hline \multirow{4}{*}{ Length $[\mathrm{mm}]$} & between groups & 7869.328 & 2 & 3934.664 & 15.544 & 0.000 \\
\cline { 2 - 7 } & within groups & 107831.823 & 426 & 253.126 & & \\
\cline { 2 - 7 } & Total & 115701.152 & 428 & & & \\
\hline \multirow{5}{*}{ Width $[\mathrm{mm}]$} & between groups & 5992.271 & 2 & 2996.136 & 14.463 & 0.000 \\
\cline { 2 - 7 } & within groups & 88248.715 & 426 & 207.157 & & \\
\cline { 2 - 7 } & Total & 94240.986 & 428 & & & \\
\hline \multirow{2}{*}{ Thickness $[\mathrm{mm}]$} & between groups & 572.511 & 2 & 286.255 & 8.459 & 0.000 \\
\cline { 2 - 8 } & within groups & 14416.532 & 426 & 33.842 & & \\
\cline { 2 - 8 } & Total & 14989.043 & 428 & & & \\
\hline
\end{tabular}

Multiple Comparisons

Tukey HSD

\begin{tabular}{|c|c|c|c|c|c|c|c|}
\hline \multirow{2}{*}{$\begin{array}{l}\text { Dependent } \\
\text { variable }\end{array}$} & \multirow{2}{*}{ (I) raw material } & \multirow{2}{*}{ (J) raw material } & \multirow{2}{*}{$\begin{array}{c}\text { Mean } \\
\text { difference } \\
(\mathrm{I}-\mathrm{J})\end{array}$} & \multirow{2}{*}{ Std. error } & \multirow{2}{*}{ Sig. } & \multicolumn{2}{|c|}{$\begin{array}{l}\text { 95\% Confidence } \\
\text { interval }\end{array}$} \\
\hline & & & & & & $\begin{array}{l}\text { Lower } \\
\text { bound }\end{array}$ & $\begin{array}{l}\text { Upper } \\
\text { bound }\end{array}$ \\
\hline \multirow{6}{*}{$\begin{array}{l}\text { Length } \\
{[\mathrm{mm}]}\end{array}$} & \multirow[t]{2}{*}{ local } & regional & 10.37875 & 2.51916 & 0.000 & 4.4538 & 16.3037 \\
\hline & & transcarpathian & 12.00945 & 2.89684 & 0.000 & 5.1963 & 18.8226 \\
\hline & \multirow[t]{2}{*}{ regional } & local & -10.37875 & 2.51916 & 0.000 & -16.3037 & -4.4538 \\
\hline & & transcarpathian & 1.63071 & 3.64630 & 0.896 & -6.9452 & 10.2066 \\
\hline & \multirow[t]{2}{*}{ transcarpathian } & local & -12.00945 & 2.89684 & 0.000 & -18.8226 & -5.1963 \\
\hline & & regional & -1.63071 & 3.64630 & 0.896 & -10.2066 & 6.9452 \\
\hline \multirow{6}{*}{$\begin{array}{l}\text { Width } \\
{[\mathrm{mm}]}\end{array}$} & \multirow[t]{2}{*}{ local } & regional & 9.19858 & 2.27896 & 0.000 & 3.8386 & 14.5585 \\
\hline & & transcarpathian & 10.31474 & 2.62062 & 0.000 & 4.1512 & 16.4783 \\
\hline & \multirow[t]{2}{*}{ regional } & local & -9.19858 & 2.27896 & 0.000 & -14.5585 & -3.8386 \\
\hline & & transcarpathian & 1.11616 & 3.29863 & 0.939 & -6.6420 & 8.8743 \\
\hline & \multirow[t]{2}{*}{ transcarpathian } & local & -10.31474 & 2.62062 & 0.000 & -16.4783 & -4.1512 \\
\hline & & regional & -1.11616 & 3.29863 & 0.939 & -8.8743 & 6.6420 \\
\hline \multirow{6}{*}{$\begin{array}{l}\text { Thickness } \\
{[\mathrm{mm}]}\end{array}$} & \multirow[t]{2}{*}{ local } & regional & 2.15846 & 0.92111 & 0.051 & -0.0079 & 4.3249 \\
\hline & & transcarpathian & 3.80816 & 1.05921 & 0.001 & 1.3170 & 6.2993 \\
\hline & \multirow[t]{2}{*}{ regional } & local & -2.15846 & 0.92111 & 0.051 & -4.3249 & 0.0079 \\
\hline & & transcarpathian & 1.64970 & 1.33324 & 0.432 & -1.4860 & 4.7854 \\
\hline & \multirow[t]{2}{*}{ transcarpathian } & local & -3.80816 & 1.05921 & 0.001 & -6.2993 & -1.3170 \\
\hline & & regional & -1.64970 & 1.33324 & 0.432 & -4.7854 & 1.4860 \\
\hline
\end{tabular}


Table 50. Bodrogkeresztúr flake tool assemblage length, width and thickness by raw materials

\begin{tabular}{|c|c|c|c|c|}
\hline \multicolumn{2}{|l|}{ Raw material } & Length [mm] & Width [mm] & Thickness [mm] \\
\hline \multirow[t]{6}{*}{ Local } & Minimum & 12.10 & 9.80 & 3.90 \\
\hline & Maximum & 139.20 & 97.00 & 35.70 \\
\hline & Mean & 45.2890 & 36.0560 & 13.2857 \\
\hline & Median & 40.6000 & 31.2000 & 11.5000 \\
\hline & $\mathrm{N}$ & 91 & 91 & 91 \\
\hline & Std. deviation & 19.96260 & 16.44593 & 6.49319 \\
\hline \multirow[t]{6}{*}{ Regional } & Minimum & 12.00 & 11.00 & 4.10 \\
\hline & Maximum & 56.00 & 39.00 & 15.50 \\
\hline & Mean & 32.3525 & 24.6000 & 8.7275 \\
\hline & Median & 31.1500 & 24.3000 & 8.2500 \\
\hline & $\mathrm{N}$ & 40 & 40 & 40 \\
\hline & Std. deviation & 9.58193 & 5.40370 & 2.79826 \\
\hline \multirow[t]{6}{*}{ Transcarpathian } & Minimum & 9.70 & 17.50 & 4.20 \\
\hline & Maximum & 63.10 & 45.80 & 16.00 \\
\hline & Mean & 35.4000 & 28.4348 & 8.7565 \\
\hline & Median & 33.3000 & 28.0000 & 8.2000 \\
\hline & $\mathrm{N}$ & 23 & 23 & 23 \\
\hline & Std. deviation & 12.31998 & 7.70222 & 2.79575 \\
\hline \multirow[t]{6}{*}{ Total } & Minimum & 9.70 & 9.80 & 3.90 \\
\hline & Maximum & 139.20 & 97.00 & 35.70 \\
\hline & Mean & 40.4519 & 31.9422 & 11.4253 \\
\hline & Median & 36.1000 & 28.0500 & 10.1500 \\
\hline & $\mathrm{N}$ & 154 & 154 & 154 \\
\hline & Std. deviation & 17.73545 & 14.18031 & 5.74045 \\
\hline
\end{tabular}

Table 51. Bodrogkeresztúr flake blank and tool length, width and thickness mean t-test comparison

\begin{tabular}{|c|c|c|c|c|c|c|c|c|}
\hline \multicolumn{9}{|c|}{ Independent Samples Test } \\
\hline \multirow{2}{*}{\multicolumn{2}{|c|}{ Raw material }} & $\begin{array}{c}\text { Leven } \\
\text { for } \mathrm{E} \\
\text { of } \mathrm{Va}\end{array}$ & $\begin{array}{l}\text { 's Test } \\
\text { uality } \\
\text { ances }\end{array}$ & \multicolumn{5}{|c|}{ t-test for Equality of Means } \\
\hline & & $\mathrm{F}$ & Sig. & $\mathrm{t}$ & df & $\begin{array}{c}\text { Sig. } \\
\text { (2-tailed) }\end{array}$ & $\begin{array}{c}\text { Mean } \\
\text { difference }\end{array}$ & $\begin{array}{l}\text { Std. error } \\
\text { difference }\end{array}$ \\
\hline \multirow[t]{6}{*}{ Local } & \multirow{2}{*}{$\begin{array}{l}\text { length } \\
{[\mathrm{mm}]}\end{array}$} & 0.708 & 0.401 & -2.778 & 440 & 0.006 & -5.76138 & 2.07363 \\
\hline & & & & -2.526 & 125.772 & 0.013 & -5.76138 & 2.28039 \\
\hline & \multirow{2}{*}{$\begin{array}{l}\text { width } \\
{[\mathrm{mm}]}\end{array}$} & 0.258 & 0.612 & -1.397 & 440 & 0.163 & -2.56858 & 1.83839 \\
\hline & & & & -1.345 & 133.856 & 0.181 & -2.56858 & 1.91018 \\
\hline & \multirow{2}{*}{$\begin{array}{l}\text { thickness } \\
{[\mathrm{mm}]}\end{array}$} & 0.968 & 0.326 & -4.672 & 440 & 0.000 & -3.44725 & 0.73788 \\
\hline & & & & -4.553 & 135.854 & 0.000 & -3.44725 & 0.75720 \\
\hline \multirow[t]{6}{*}{ Regional } & \multirow{2}{*}{$\begin{array}{l}\text { length } \\
{[\mathrm{mm}]}\end{array}$} & 0.752 & 0.388 & -1.431 & 83 & 0.156 & -3.20361 & 2.23822 \\
\hline & & & & -1.442 & 82.993 & 0.153 & -3.20361 & 2.22125 \\
\hline & \multirow{2}{*}{$\begin{array}{l}\text { width } \\
{[\mathrm{mm}]}\end{array}$} & 6.686 & 0.011 & -0.206 & 83 & 0.837 & -0.31111 & 1.51112 \\
\hline & & & & -0.211 & 77.317 & 0.834 & -0.31111 & 1.47713 \\
\hline & \multirow{2}{*}{$\begin{array}{l}\text { thickness } \\
{[\mathrm{mm}]}\end{array}$} & 1.397 & 0.241 & -1.373 & 83 & 0.173 & -1.04750 & 0.76289 \\
\hline & & & & -1.402 & 78.523 & 0.165 & -1.04750 & 0.74711 \\
\hline \multirow[t]{6}{*}{ Transcarpathian } & \multirow{2}{*}{$\begin{array}{l}\text { length } \\
{[\mathrm{mm}]}\end{array}$} & 3.084 & 0.085 & -2.989 & 54 & 0.004 & -7.88182 & 2.63677 \\
\hline & & & & -2.743 & 33.002 & 0.010 & -7.88182 & 2.87329 \\
\hline & \multirow{2}{*}{$\begin{array}{l}\text { width } \\
{[\mathrm{mm}]}\end{array}$} & 0.001 & 0.974 & -2.386 & 54 & 0.021 & -5.26206 & 2.20540 \\
\hline & & & & -2.423 & 49.961 & 0.019 & -5.26206 & 2.17127 \\
\hline & \multirow{2}{*}{$\begin{array}{l}\text { thickness } \\
{[\mathrm{mm}]}\end{array}$} & 0.928 & 0.340 & -3.923 & 54 & 0.000 & -2.72622 & 0.69489 \\
\hline & & & & -3.811 & 42.416 & 0.000 & -2.72622 & 0.71531 \\
\hline
\end{tabular}


Table 52. Bodrogkeresztúr flake tool length, width and thickness mean comparison by raw materials with ANOVA and the Tukey post hoc

ANOVA

\begin{tabular}{|l|l|r|r|r|r|c|}
\hline \multicolumn{2}{|c|}{} & Sum of squares & df & Mean square & F & Sig. \\
\hline \multirow{3}{*}{ Length $[\mathrm{mm}]$} & between groups & 5340.196 & 2 & 2670.098 & 9.423 & 0.000 \\
\cline { 2 - 7 } & within groups & 42785.389 & 151 & 283.347 & & \\
\cline { 2 - 7 } & Total & 48125.584 & 153 & & & \\
\hline \multirow{3}{*}{ Width [mm] } & between groups & 3979.319 & 2 & 1989.660 & 11.216 & 0.000 \\
\cline { 2 - 7 } & within groups & 26786.096 & 151 & 177.391 & & \\
\cline { 2 - 7 } & Total & 30765.416 & 153 & & & \\
\hline \multirow{2}{*}{ Thickness [mm] } & between groups & 769.904 & 2 & 384.952 & 13.607 & 0.000 \\
\cline { 2 - 7 } & within groups & 4271.868 & 151 & 28.291 & & \\
\cline { 2 - 7 } & Total & 5041.771 & 153 & & & \\
\hline
\end{tabular}

Multiple Comparisons

Tukey HSD

\begin{tabular}{|c|c|c|c|c|c|c|c|}
\hline \multirow{2}{*}{$\begin{array}{l}\text { Dependent } \\
\text { variable }\end{array}$} & \multirow{2}{*}{ (I) raw material } & \multirow{2}{*}{ (J) raw material } & \multirow{2}{*}{$\begin{array}{c}\text { Mean } \\
\text { difference } \\
\quad(\mathrm{I}-\mathrm{J})\end{array}$} & \multirow{2}{*}{$\begin{array}{l}\text { Std. } \\
\text { error }\end{array}$} & \multirow{2}{*}{ Sig. } & \multicolumn{2}{|c|}{$\begin{array}{l}\text { 95\% Confidence } \\
\text { interval }\end{array}$} \\
\hline & & & & & & $\begin{array}{l}\text { Lower } \\
\text { bound }\end{array}$ & $\begin{array}{l}\text { Upper } \\
\text { bound }\end{array}$ \\
\hline \multirow{6}{*}{$\begin{array}{l}\text { Length } \\
{[\mathrm{mm}]}\end{array}$} & \multirow[t]{2}{*}{ local } & regional & 12.93651 & 3.19333 & 0.000 & 5.3777 & 20.4953 \\
\hline & & transcarpathian & 9.88901 & 3.92850 & 0.034 & 0.5900 & 19.1880 \\
\hline & \multirow[t]{2}{*}{ regional } & local & -12.93651 & 3.19333 & 0.000 & -20.4953 & -5.3777 \\
\hline & & transcarpathian & -3.04750 & 4.40490 & 0.769 & -13.4741 & 7.3791 \\
\hline & \multirow[t]{2}{*}{ transcarpathian } & local & -9.88901 & 3.92850 & 0.034 & -19.1880 & -0.5900 \\
\hline & & regional & 3.04750 & 4.40490 & 0.769 & -7.3791 & 13.4741 \\
\hline \multirow{6}{*}{$\begin{array}{l}\text { Width } \\
{[\mathrm{mm}]}\end{array}$} & \multirow[t]{2}{*}{ local } & regional & 11.45604 & 2.52669 & 0.000 & 5.4752 & 17.4368 \\
\hline & & transcarpathian & 7.62126 & 3.10838 & 0.040 & 0.2636 & 14.9790 \\
\hline & \multirow[t]{2}{*}{ regional } & local & -11.45604 & 2.52669 & 0.000 & -17.4368 & -5.4752 \\
\hline & & transcarpathian & -3.83478 & 3.48532 & 0.515 & -12.0847 & 4.4152 \\
\hline & \multirow[t]{2}{*}{ transcarpathian } & local & -7.62126 & 3.10838 & 0.040 & -14.9790 & -0.2636 \\
\hline & & \begin{tabular}{|l|} 
regional \\
\end{tabular} & 3.83478 & 3.48532 & 0.515 & -4.4152 & 12.0847 \\
\hline \multirow{6}{*}{$\begin{array}{l}\text { Thickness } \\
{[\mathrm{mm}]}\end{array}$} & \multirow[t]{2}{*}{ local } & regional & 4.55821 & 1.00903 & 0.000 & 2.1698 & 6.9466 \\
\hline & & transcarpathian & 4.52919 & 1.24133 & 0.001 & 1.5909 & 7.4675 \\
\hline & \multirow[t]{2}{*}{ regional } & local & -4.55821 & 1.00903 & 0.000 & -6.9466 & -2.1698 \\
\hline & & transcarpathian & -0.02902 & 1.39186 & 1.000 & -3.3236 & 3.2656 \\
\hline & \multirow[t]{2}{*}{ transcarpathian } & local & -4.52919 & 1.24133 & 0.001 & -7.4675 & -1.5909 \\
\hline & & regional & 0.02902 & 1.39186 & 1.000 & -3.2656 & 3.3236 \\
\hline
\end{tabular}


Table 53. Bodrogkeresztúr flake tool types by raw material

\begin{tabular}{|c|c|c|c|c|c|c|}
\hline & \multicolumn{3}{|c|}{ Raw material } & \multirow{2}{*}{ Total } \\
\hline & & & local & regional & transcarpathian & \\
\hline \multirow{16}{*}{ Tooltypes } & \multirow[t]{2}{*}{ endscraper } & count & 27 & 14 & 6 & 47 \\
\hline & & $\%$ within raw material & $29.7 \%$ & $35.0 \%$ & $26.1 \%$ & $30.5 \%$ \\
\hline & \multirow[t]{2}{*}{ burin } & count & 21 & 6 & 12 & 39 \\
\hline & & \% within raw material & $23.1 \%$ & $15.0 \%$ & $52.2 \%$ & $25.3 \%$ \\
\hline & \multirow[t]{2}{*}{ retouched } & count & 23 & 12 & 1 & 36 \\
\hline & & $\%$ within raw material & $25.3 \%$ & $30.0 \%$ & $4.3 \%$ & $23.4 \%$ \\
\hline & \multirow[t]{2}{*}{ borer } & count & 2 & 0 & 0 & 2 \\
\hline & & $\%$ within raw material & $2.2 \%$ & $0.0 \%$ & $0.0 \%$ & $1.3 \%$ \\
\hline & \multirow{2}{*}{$\begin{array}{l}\text { splintered } \\
\text { piece }\end{array}$} & count & 1 & 1 & 3 & 5 \\
\hline & & $\%$ within raw material & $1.1 \%$ & $2.5 \%$ & $13.0 \%$ & $3.2 \%$ \\
\hline & \multirow[t]{2}{*}{ truncation } & count & 0 & 3 & 0 & 3 \\
\hline & & $\%$ within raw material & $0.0 \%$ & $7.5 \%$ & $0.0 \%$ & $1.9 \%$ \\
\hline & \multirow[t]{2}{*}{ composite } & count & 2 & 0 & 1 & 3 \\
\hline & & $\%$ within raw material & $2.2 \%$ & $0.0 \%$ & $4.3 \%$ & $1.9 \%$ \\
\hline & \multirow{2}{*}{$\begin{array}{l}\text { notched- } \\
\text { denticulated }\end{array}$} & count & 15 & 4 & 0 & 19 \\
\hline & & $\%$ within raw material & $16.5 \%$ & $10.0 \%$ & $0.0 \%$ & $12.3 \%$ \\
\hline \multirow{2}{*}{\multicolumn{2}{|c|}{ Total }} & count & 91 & 40 & 23 & 154 \\
\hline & & $\%$ within raw material & $100.0 \%$ & $100.0 \%$ & $100.0 \%$ & $100.0 \%$ \\
\hline
\end{tabular}

Table 54. Bodrogkeresztúr rejuvenating flake and debris tool types

\begin{tabular}{|c|c|c|c|c|c|c|c|}
\hline & \multicolumn{3}{|c|}{ Raw material } & \multirow{2}{*}{ Total } \\
\hline & & & & local & regional & transcarpathian & \\
\hline \multirow[t]{10}{*}{ Debris } & \multirow{8}{*}{ 总 } & \multirow[t]{2}{*}{ burin } & count & 7 & 0 & & 7 \\
\hline & & & $\%$ within raw material & $63.6 \%$ & $0.0 \%$ & & $58.3 \%$ \\
\hline & & \multirow[t]{2}{*}{ retouched } & count & 2 & 0 & & 2 \\
\hline & & & $\%$ within raw material & $18.2 \%$ & $0.0 \%$ & & $16.7 \%$ \\
\hline & & \multirow{2}{*}{$\begin{array}{l}\text { splintered } \\
\text { piece }\end{array}$} & count & 0 & 1 & & 1 \\
\hline & & & $\%$ within raw material & $0.0 \%$ & $100.0 \%$ & & $8.3 \%$ \\
\hline & & \multirow{2}{*}{$\begin{array}{l}\text { notched- } \\
\text { denticulated }\end{array}$} & count & 2 & 0 & & 2 \\
\hline & & & $\%$ within raw material & $18.2 \%$ & $0.0 \%$ & & $16.7 \%$ \\
\hline & \multirow[t]{2}{*}{ total } & & count & 11 & 1 & & 12 \\
\hline & & & $\%$ within raw material & $100.0 \%$ & $100.0 \%$ & & $100.0 \%$ \\
\hline \multirow{10}{*}{$\begin{array}{l}\text { Rejuvenating } \\
\text { flake }\end{array}$} & \multirow{8}{*}{$\frac{\mathscr{E}}{\stackrel{\Xi}{\Xi}}$} & \multirow[t]{2}{*}{ endscraper } & count & 0 & 0 & 1 & 1 \\
\hline & & & $\%$ within raw material & $0.0 \%$ & $0.0 \%$ & $50.0 \%$ & $20.0 \%$ \\
\hline & & \multirow[t]{2}{*}{ burin } & count & 0 & 1 & 1 & 2 \\
\hline & & & $\%$ within raw material & $0.0 \%$ & $100.0 \%$ & $50.0 \%$ & $40.0 \%$ \\
\hline & & \multirow[t]{2}{*}{ retouched } & count & 1 & 0 & 0 & 1 \\
\hline & & & $\%$ within raw material & $50.0 \%$ & $0.0 \%$ & $0.0 \%$ & $20.0 \%$ \\
\hline & & \multirow[t]{2}{*}{ borer } & count & 1 & 0 & 0 & 1 \\
\hline & & & $\%$ within raw material & $50.0 \%$ & $0.0 \%$ & $0.0 \%$ & $20.0 \%$ \\
\hline & \multirow{2}{*}{\multicolumn{2}{|c|}{ total }} & count & 2 & 1 & 2 & 5 \\
\hline & & & $\%$ within raw material & $100.0 \%$ & $100.0 \%$ & $100.0 \%$ & $100.0 \%$ \\
\hline
\end{tabular}




\section{HIDASNÉMETI}

The site

The site is situated on the right bank terrace of river Hernád in northeast Hungary at an elevation of $190 \mathrm{~m}$ a.s.l. facing east towards river Hernád. After field surveys in 1982, the excavations were carried out between 1983 and 1985 and ten trenches recovered 150 square meters of the site (Simán 1989).

The site stratigraphy consisted of five layers: 1) the recent topsoil $20 \mathrm{~cm}, 2$ ) a red sandy clay $25 \mathrm{~cm}, 3$ ) a brown sandy clay $10-50 \mathrm{~cm}, 4$ ) a yellow hard clay of undefined thickness and 5) the gravel of the tertiary Hernád terrace. Two main levels of artifact were claimed to had been found in trenches VII-IX, but the exact stratigraphic position of these levels and the elevation difference between them remained unknown. Other trenches yielded finds throughout the whole sequence. Today it is impossible to separate all finds from the two levels because many has lost the inventory number that would indicate the archaeological level.

Besides the lithics, Simán (1989) described three hearts, charred residues of four pointed wooden sticks, and two postholes of 10 and $20 \mathrm{~cm}$ diameter in the upper level. In the lower level two hearths were found, one of which was hewn into the ground $15 \mathrm{~cm}$ deep on a surface of $20 \times 40 \mathrm{~cm}$ and $90 \times 30 \mathrm{~cm}$, respectively. Both contained calcined bones but charcoals. Also, a chunk of charred wood was fund in this level. The preservation of the bones was poor, thus none of them was identified to species, and the wood remains were not saved and analyzed either.

The dating of the site solely relies on the typology of the shouldered points which are typical to the Late Gravettian or Willendorf-Kostenkian dated elsewhere to 25-21 ka BP (SimÁn 1989; Lengyel 2014a). All the knapped lithics were analyzed here.

\section{Raw materials}

The assemblage almost entirely consists of regional limnic silicite (Tab. 55). The most popular type is the white variant that has a fine and heterogeneous body texture. Further types are brown in original colour, but the patina turns the colours into reddish, blue and white. These colours are often stripped on the materials. A third type is a greyish-blue silicite. Further materials present with a few items are the obsidian Carpathian 2 type, radiolarite, and the meta-rhyolite of Bükk Mountains. Regional materials yielded 134.9 items per $\mathrm{kg}$. Raw material from the longest distance is the Cretaceous flint, which provided 22.2 items per $\mathrm{kg}$.

\section{Blade tool production}

The technological composition of the assemblage showed that only the regional materials went through a complete processing, providing all the elements of a laminar debitage, except the crest blade (Tab. 56). Transcarpathian materials yielded only two flakes and two blades. 
The blade technology primarily used unidirectional debitage (Tabs 57, 58), and often adjusted the striking platform of the core before detaching a blade with a few minute flake removals (Tab. 59). Soft hammer technique was dominant in blade debitage (Tab. 60) which produced products between 20 and $110 \mathrm{~mm}$ (Tab. 61).

The majority of the tools $(80.0 \%)$ were made of blades (Tab. 62). Out of the total number of regional blades, $8.1 \%$ was used up for tools, while this ratio is much higher for transcarpathian blades, from which both specimens are tools (Tab. 63).

The mean length of the blade tools shows that regional specimens are similar to the transcarpathian ones (Tab. 64). The t-test found that blade tools (only regional items are involved) are shorter, but wider and thicker than blank blades (Tab. 65), and there is no difference between regional and transcarpathian blade tools in length, width and thickness (Tab. 66).

Most of the blade tools are burins and armature (Tab. 67). TC blade tools are a burin and a composite tool. The armatures are chiefly backed bladelets and points (Tab. 68). Among the points the shouldered, Gravette and tip retouched blades are present with more than one specimen, and the fléchette has a single occurrence (Tab. 69).

\section{Flake tool production}

The evidences of flake debitage are the few cores, and a half of them has multiplatform (Tab. 70). On the flakes unidirectional scars are prevalent (Tab. 71), plain butts are frequent (Tab. 72), and many of them have well visible impact points that are signs of hard hammer technique (Tab. 73). The mean size of the flakes is rather small (Tab. 74).

The flakes are the minority in the tools (Tab. 62) and only a small portion of the total flake assemblage was used up for tools (Tab. 63). Flake tool sizes differ from the blanks by the former being longer and thicker (Tabs 75, 76).

Most of the flake tools are edge retouched types, and endscrapers, burins, and notchdenticulates are in equal share (Tab. 77). A few tools were made of debris. These are two burins and one notched-denticulated type made of local material. There is a single notched-denticulated item made of a rejuvenating flake (Tab. 78).

Table 55. Hidasnémeti lithic raw material composition by weight in grams

\begin{tabular}{|l|c|c|c|c|c|c|}
\hline Raw material & Flake & Blade & Debris & Core & Total & $\%$ \\
\hline Regional & 7861 & 7109 & 3295 & 4634 & 22899 & 99.9 \\
\hline Within regional \% & 34.32901 & 31.04502 & 14.38927 & 20.23669 & 100 & \\
\hline Transcarpathian & & 18 & & & 18 & 0.1 \\
\hline Within transcarpathian \% & & 100 & & & 100 & \\
\hline Total & 7861 & 7127 & 3295 & 4634 & 22917 & \\
\hline$\%$ & 34.30205 & 31.09918 & 14.37797 & 20.2208 & 100 & \\
\hline
\end{tabular}


Table 56. Hidasnémeti lithic assemblage composition by raw material types and technological categories

\begin{tabular}{|c|c|c|c|c|c|}
\hline & \multicolumn{2}{|c|}{ Raw material } & \multirow{2}{*}{ Total } \\
\hline & & & regional & transcarpathian & \\
\hline \multirow[t]{16}{*}{ Class } & \multirow[t]{2}{*}{ flake } & count & 1076 & 2 & 1078 \\
\hline & & $\%$ within raw material & $34.8 \%$ & $50.0 \%$ & $34.9 \%$ \\
\hline & \multirow[t]{2}{*}{ blade } & count & 1146 & 2 & 1148 \\
\hline & & $\%$ within raw material & $37.1 \%$ & $50.0 \%$ & $37.1 \%$ \\
\hline & \multirow[t]{2}{*}{ debris } & count & 546 & 0 & 546 \\
\hline & & $\%$ within raw material & $17.7 \%$ & $0.0 \%$ & $17.7 \%$ \\
\hline & \multirow[t]{2}{*}{ rejuvenating flake } & count & 160 & 0 & 160 \\
\hline & & $\%$ within raw material & $5.2 \%$ & $0.0 \%$ & $5.2 \%$ \\
\hline & \multirow[t]{2}{*}{ crest } & count & 22 & 0 & 22 \\
\hline & & $\%$ within raw material & $0.7 \%$ & $0.0 \%$ & $0.7 \%$ \\
\hline & \multirow[t]{2}{*}{ neo-crest } & count & 86 & 0 & 86 \\
\hline & & $\%$ within raw material & $2.8 \%$ & $0.0 \%$ & $2.8 \%$ \\
\hline & \multirow{2}{*}{ blade core } & count & 47 & 0 & 47 \\
\hline & & $\%$ within raw material & $1.5 \%$ & $0.0 \%$ & $1.5 \%$ \\
\hline & \multirow[t]{2}{*}{ flake core } & count & 6 & 0 & 6 \\
\hline & & $\%$ within raw material & $0.2 \%$ & $0.0 \%$ & $0.2 \%$ \\
\hline \multirow{2}{*}{\multicolumn{2}{|c|}{ Total }} & count & 3089 & 4 & 3093 \\
\hline & & $\%$ within raw material & $100.0 \%$ & $100.0 \%$ & $100.0 \%$ \\
\hline
\end{tabular}

Table 57. Hidasnémeti blade assemblage (complete specimens) dorsal scar pattern frequency by raw material

\begin{tabular}{|c|c|c|c|c|}
\hline & Raw material & \multirow{2}{*}{ Total } \\
\hline & & & regional & \\
\hline \multirow[t]{6}{*}{ Scars } & \multirow[t]{2}{*}{ unidirectional } & count & 48 & 48 \\
\hline & & $\%$ within raw material & $56.5 \%$ & $56.5 \%$ \\
\hline & \multirow[t]{2}{*}{ opposite } & count & 20 & 20 \\
\hline & & $\%$ within raw material & $23.5 \%$ & $23.5 \%$ \\
\hline & \multirow{2}{*}{ perpendicular } & count & 17 & 17 \\
\hline & & \% within raw material & $20.0 \%$ & $20.0 \%$ \\
\hline \multirow{2}{*}{\multicolumn{2}{|c|}{ Total }} & count & 85 & 85 \\
\hline & & \% within raw material & $100.0 \%$ & $100.0 \%$ \\
\hline
\end{tabular}

Table 58. Hidasnémeti blade core types by raw material

\begin{tabular}{|c|c|c|c|c|}
\hline & \multirow{3}{*}{$\begin{array}{c}\text { Raw material } \\
\text { regional } \\
32\end{array}$} & \multirow{3}{*}{$\begin{array}{c}\text { Total } \\
32 \\
\end{array}$} \\
\hline & & & & \\
\hline \multirow[t]{10}{*}{ Types } & \multirow[t]{2}{*}{ unidirectional } & count & & \\
\hline & & $\%$ within raw material & $68.1 \%$ & $68.1 \%$ \\
\hline & \multirow[t]{2}{*}{ bidirectional } & count & 2 & 2 \\
\hline & & $\%$ within raw material & $4.3 \%$ & $4.3 \%$ \\
\hline & \multirow[t]{2}{*}{ alternate } & count & 2 & 2 \\
\hline & & $\%$ within raw material & $4.3 \%$ & $4.3 \%$ \\
\hline & \multirow[t]{2}{*}{ multidirectional } & count & 2 & 2 \\
\hline & & $\%$ within raw material & $4.3 \%$ & $4.3 \%$ \\
\hline & \multirow[t]{2}{*}{ core fragment } & count & 9 & 9 \\
\hline & & $\%$ within raw material & $19.1 \%$ & $19.1 \%$ \\
\hline \multirow{2}{*}{\multicolumn{2}{|c|}{ Total }} & count & 47 & 47 \\
\hline & & \% within raw material & $100.0 \%$ & $100.0 \%$ \\
\hline
\end{tabular}


Table 59. Hidasnémeti blade assemblage (complete specimens) platform type frequency by raw material

\begin{tabular}{|c|c|c|c|c|}
\hline & Raw material & \multirow{2}{*}{ Total } \\
\hline & & & regional & \\
\hline \multirow[t]{12}{*}{ Platform } & \multirow[t]{2}{*}{ plain } & count & 38 & 38 \\
\hline & & $\%$ within raw material & $44.7 \%$ & $44.7 \%$ \\
\hline & \multirow[t]{2}{*}{ dihedral } & count & 14 & 14 \\
\hline & & $\%$ within raw material & $16.5 \%$ & $16.5 \%$ \\
\hline & \multirow[t]{2}{*}{ faceted } & count & 28 & 28 \\
\hline & & $\%$ within raw material & $32.9 \%$ & $32.9 \%$ \\
\hline & \multirow[t]{2}{*}{ linear } & count & 2 & 2 \\
\hline & & $\%$ within raw material & $2.4 \%$ & $2.4 \%$ \\
\hline & \multirow[t]{2}{*}{ irregular } & count & 1 & 1 \\
\hline & & $\%$ within raw material & $1.2 \%$ & $1.2 \%$ \\
\hline & \multirow[t]{2}{*}{ spur } & count & 2 & 2 \\
\hline & & $\%$ within raw material & $2.4 \%$ & $2.4 \%$ \\
\hline \multirow{2}{*}{\multicolumn{2}{|c|}{ Total }} & count & 85 & 85 \\
\hline & & $\%$ within raw material & $100.0 \%$ & $100.0 \%$ \\
\hline
\end{tabular}

Table 60. Hidasnémeti blade assemblage (complete specimens) impact point-overhang co-presence by raw material

\begin{tabular}{|c|c|c|c|c|}
\hline & Raw material & \multirow{2}{*}{ Total } \\
\hline & & & regional & \\
\hline \multirow{6}{*}{$\begin{array}{l}\text { Impact } \\
\text { point-overhang }\end{array}$} & \multirow[t]{2}{*}{ none } & count & 75 & 75 \\
\hline & & $\%$ within raw material & $88.2 \%$ & $88.2 \%$ \\
\hline & \multirow[t]{2}{*}{ yes-no } & count & 1 & 1 \\
\hline & & $\%$ within raw material & $1.2 \%$ & $1.2 \%$ \\
\hline & \multirow[t]{2}{*}{ no-yes } & count & 9 & 9 \\
\hline & & $\%$ within raw material & $10.6 \%$ & $10.6 \%$ \\
\hline \multirow{2}{*}{\multicolumn{2}{|c|}{ Total }} & count & 85 & 85 \\
\hline & & $\%$ within raw material & $100.0 \%$ & $100.0 \%$ \\
\hline
\end{tabular}

Table 61. Hidasnémeti blade assemblage (complete specimens) length, width and thickness by raw material

\begin{tabular}{|l|l|c|c|c|}
\hline Raw material & & Length [mm] & Width [mm] & Thickness [mm] \\
\hline \multirow{5}{*}{ Regional } & Minimum & 19.30 & 6.80 & 2.00 \\
\cline { 2 - 5 } & Maximum & 111.50 & 37.31 & 17.30 \\
\cline { 2 - 5 } & Mean & 58.6071 & 20.1046 & 6.9004 \\
\cline { 2 - 5 } & Median & 55.0000 & 19.3000 & 6.9000 \\
\cline { 2 - 5 } & N & 85 & 85 & 85 \\
\cline { 2 - 5 } & Std. deviation & 20.84511 & 6.03526 & 2.86517 \\
\hline \multirow{5}{*}{ Total } & Minimum & 19.30 & 6.80 & 2.00 \\
\cline { 2 - 5 } & Maximum & 111.50 & 37.31 & 17.30 \\
\cline { 2 - 5 } & Mean & 58.6071 & 20.1046 & 6.9004 \\
\cline { 2 - 5 } & Median & 55.0000 & 19.3000 & 6.9000 \\
\cline { 2 - 5 } & N & 85 & 85 & 2.86517 \\
\cline { 2 - 5 } & Std. deviation & 20.84511 & 6.03526 & \\
\hline
\end{tabular}


Table 62. Hidasnémeti tool assemblage product composition by raw materials

\begin{tabular}{|l|l|c|c|c|}
\hline \multicolumn{2}{|c|}{} & \multicolumn{2}{|c|}{ Raw material } & \multirow{2}{*}{ Total } \\
\cline { 3 - 5 } \multicolumn{2}{|c|}{ Flake } & regional & transcarpathian & \\
\cline { 2 - 5 } & $\%$ within raw material & $17.2 \%$ & $0.0 \%$ & $16.9 \%$ \\
\hline \multirow{2}{*}{ Blade } & count & 102 & 2 & 104 \\
\cline { 2 - 5 } & $\%$ within raw material & $79.7 \%$ & $100.0 \%$ & $80.0 \%$ \\
\hline \multirow{2}{*}{ Debris } & count & 3 & 0 & 3 \\
\cline { 2 - 5 } & $\%$ within raw material & $2.3 \%$ & $0.0 \%$ & $2.3 \%$ \\
\hline $\begin{array}{l}\text { Rejuvenating } \\
\text { flake }\end{array}$ & count & 1 & 0 & 1 \\
\hline \multirow{2}{*}{ Total } & $\%$ within raw material & $0.8 \%$ & $0.0 \%$ & $0.8 \%$ \\
\cline { 2 - 5 } & count & 128 & 2 & 130 \\
\cline { 2 - 5 } & $\%$ within raw material & $100.0 \%$ & $100.0 \%$ & $100.0 \%$ \\
\hline
\end{tabular}

Table 63. Hidasnémeti knapped product frequency in tool assemblage by raw materials

\begin{tabular}{|c|c|c|c|c|c|c|}
\hline & & & & & material & Total \\
\hline & & & & regional & transcarpathian & Iotal \\
\hline Flake & state & blank & count & 1054 & 2 & 1056 \\
\hline & & & $\%$ within raw material & $98.0 \%$ & $100.0 \%$ & $98.0 \%$ \\
\hline & & tool & count & 22 & 0 & 22 \\
\hline & & & $\%$ within raw material & $2.0 \%$ & $0.0 \%$ & $2.0 \%$ \\
\hline & total & & count & 1076 & 2 & 1078 \\
\hline & & & $\%$ within raw material & $100.0 \%$ & $100.0 \%$ & $100.0 \%$ \\
\hline Blade & state & blank & count & 1152 & 0 & 1152 \\
\hline & & & $\%$ within raw material & $91.9 \%$ & $0.0 \%$ & $91.7 \%$ \\
\hline & & tool & count & 102 & 2 & 104 \\
\hline & & & $\%$ within raw material & $8.1 \%$ & $100.0 \%$ & $8.3 \%$ \\
\hline & total & & count & 1254 & 2 & 1256 \\
\hline & & & $\%$ within raw material & $100.0 \%$ & $100.0 \%$ & $100.0 \%$ \\
\hline Debris & state & blank & count & 543 & & 543 \\
\hline & & & $\%$ within raw material & $99.5 \%$ & & $99.5 \%$ \\
\hline & & tool & count & 3 & & 3 \\
\hline & & & $\%$ within raw material & $0.5 \%$ & & $0.5 \%$ \\
\hline & total & & count & 546 & & 546 \\
\hline & & & $\%$ within raw material & $100.0 \%$ & & $100.0 \%$ \\
\hline Rejuvenating & state & blank & count & 159 & & 159 \\
\hline flake & & & $\%$ within raw material & $99.4 \%$ & & $99.4 \%$ \\
\hline & & tool & count & 1 & & 1 \\
\hline & & & $\%$ within raw material & $0.6 \%$ & & $0.6 \%$ \\
\hline & total & & count & 160 & & 160 \\
\hline & & & $\%$ within raw material & $100.0 \%$ & & $100.0 \%$ \\
\hline
\end{tabular}


Table 64. Hidasnémeti blade tool assemblage length, width and thickness by raw materials

\begin{tabular}{|c|c|c|c|c|}
\hline \multicolumn{2}{|l|}{ Raw material } & Length [mm] & Width [mm] & Thickness [mm] \\
\hline \multirow[t]{6}{*}{ Regional } & Minimum & 3.40 & 5.72 & 1.50 \\
\hline & Maximum & 94.90 & 49.90 & 28.00 \\
\hline & Mean & 40.7355 & 22.2472 & 9.1966 \\
\hline & Median & 39.0500 & 21.9500 & 8.7000 \\
\hline & $\mathrm{N}$ & 102 & 102 & 102 \\
\hline & Std. deviation & 17.99811 & 9.86050 & 5.03526 \\
\hline \multirow{6}{*}{ Transcarpathian } & Minimum & 38.00 & 18.80 & 11.20 \\
\hline & Maximum & 42.40 & 23.60 & 11.30 \\
\hline & Mean & 40.2000 & 21.2000 & 11.2500 \\
\hline & Median & 40.2000 & 21.2000 & 11.2500 \\
\hline & $\mathrm{N}$ & 2 & 2 & 2 \\
\hline & Std. deviation & 3.11127 & 3.39411 & 0.07071 \\
\hline \multirow[t]{6}{*}{ Total } & Minimum & 3.40 & 5.72 & 1.50 \\
\hline & Maximum & 94.90 & 49.90 & 28.00 \\
\hline & Mean & 40.7252 & 22.2270 & 9.2361 \\
\hline & Median & 39.0500 & 21.9500 & 8.8350 \\
\hline & $\mathrm{N}$ & 104 & 104 & 104 \\
\hline & Std. deviation & 17.82531 & 9.77109 & 4.99418 \\
\hline
\end{tabular}

Table 65. Hidasnémeti blade blank and tool length, width and thickness mean t-test comparison for regional raw material

\begin{tabular}{|l|c|c|r|r|r|r|r|}
\hline \multicolumn{1}{|c|}{$\begin{array}{l}\text { Levene's Test } \\
\text { for Equality } \\
\text { of Variances }\end{array}$} & \multicolumn{6}{|c|}{ Independent Samples Test } \\
\cline { 2 - 8 } & $\mathrm{F}$ & Sig. & $\mathrm{t}$ & $\mathrm{df}$ & $\begin{array}{c}\text { Sig. } \\
\text { (2-tailed) }\end{array}$ & $\begin{array}{c}\text { Mean } \\
\text { difference }\end{array}$ & $\begin{array}{c}\text { Std. error } \\
\text { difference }\end{array}$ \\
\cline { 2 - 8 } & 2.012 & 0.158 & 6.291 & 185 & 0.000 & 17.87157 & 2.84074 \\
\hline $\begin{array}{l}\text { Length } \\
{[\mathrm{mm}]}\end{array}$ & & & 6.208 & 167.140 & 0.000 & 17.87157 & 2.87885 \\
\hline $\begin{array}{l}\text { Width } \\
{[\mathrm{mm}]}\end{array}$ & 20.262 & 0.000 & -1.748 & 185 & 0.082 & -2.14257 & 1.22541 \\
\hline $\begin{array}{l}\text { Thickness } \\
{[\mathrm{mm}]}\end{array}$ & 16.699 & 0.000 & -3.730 & 185 & 0.000 & -2.29622 & 0.61558 \\
\cline { 2 - 8 } & & & -3.909 & 164.816 & 0.000 & -2.29622 & 0.58749 \\
\hline
\end{tabular}

Table 66. Hidasnémeti blade tool length, width and thickness mean comparison by raw materials with t-test

\begin{tabular}{|l|c|c|r|r|r|r|r|}
\hline \multicolumn{1}{|c|}{} & $\begin{array}{c}\text { Levene's Test } \\
\text { for Equality } \\
\text { of Variances }\end{array}$ & \multicolumn{7}{|c|}{ Independent Samples Test } \\
\cline { 2 - 8 } & $\mathrm{F}$ & Sig. & $\mathrm{t}$ & $\mathrm{df}$ & Sig. (2-tailed) & $\begin{array}{c}\text { Mean } \\
\text { difference }\end{array}$ & $\begin{array}{c}\text { Std. error } \\
\text { difference }\end{array}$ \\
\cline { 2 - 8 } & 2.383 & 0.126 & 0.042 & 102 & 0.967 & 0.53549 & 12.78949 \\
\hline $\begin{array}{l}\text { Length } \\
{[\mathrm{mm}]}\end{array}$ & 1.860 & 0.176 & 0.189 & 2.731 & 0.863 & 0.53549 & 2.83122 \\
\hline $\begin{array}{l}\text { Width } \\
{[\mathrm{mm}]}\end{array}$ & & & 0.404 & 1.352 & 0.882 & 1.04716 & 7.00996 \\
\hline $\begin{array}{l}\text { Thickness } \\
{[\mathrm{mm}]}\end{array}$ & 2.842 & 0.095 & -0.574 & 102 & 0.567 & 1.04716 & 2.59099 \\
\cline { 2 - 8 } & & & -4.098 & 102.000 & 0.000 & -2.05343 & 3.57754 \\
\hline
\end{tabular}


Table 67. Hidasnémeti blade tool types by raw material

\begin{tabular}{|c|c|c|c|c|c|}
\hline & \multicolumn{2}{|c|}{ Raw material } & \multirow{2}{*}{ Total } \\
\hline & & & regional & transcarpathian & \\
\hline \multirow[t]{14}{*}{ Tooltypes } & \multirow[t]{2}{*}{ endscraper } & count & 14 & 0 & 14 \\
\hline & & $\%$ within raw material & $13.7 \%$ & $0.0 \%$ & $13.5 \%$ \\
\hline & \multirow[t]{2}{*}{ burin } & count & 37 & 1 & 38 \\
\hline & & $\%$ within raw material & $36.3 \%$ & $50.0 \%$ & $36.5 \%$ \\
\hline & \multirow[t]{2}{*}{ retouched } & count & 17 & 0 & 17 \\
\hline & & $\%$ within raw material & $16.7 \%$ & $0.0 \%$ & $16.3 \%$ \\
\hline & \multirow[t]{2}{*}{ truncation } & count & 4 & 0 & 4 \\
\hline & & $\%$ within raw material & $3.9 \%$ & $0.0 \%$ & $3.8 \%$ \\
\hline & \multirow{2}{*}{\begin{tabular}{|l|} 
notched- \\
denticulated
\end{tabular}} & count & 1 & 0 & 1 \\
\hline & & $\%$ within raw material & $1.0 \%$ & $0.0 \%$ & $1.0 \%$ \\
\hline & \multirow[t]{2}{*}{ composite } & count & 0 & 1 & 1 \\
\hline & & $\%$ within raw material & $0.0 \%$ & $50.0 \%$ & $1.0 \%$ \\
\hline & \multirow[t]{2}{*}{ armature } & count & 29 & 0 & 29 \\
\hline & & $\%$ within raw material & $28.4 \%$ & $0.0 \%$ & $27.9 \%$ \\
\hline \multirow{2}{*}{\multicolumn{2}{|c|}{ Total }} & count & 102 & 2 & 104 \\
\hline & & $\%$ within raw material & $100.0 \%$ & $100.0 \%$ & $100.0 \%$ \\
\hline
\end{tabular}

Table 68 . Hidasnémeti armature types by raw material

\begin{tabular}{|c|c|c|c|c|}
\hline & & & Raw material & Total \\
\hline Armatures & backed & count & 16 & 16 \\
\hline & & $\%$ within raw material & $55.2 \%$ & $55.2 \%$ \\
\hline & backed- & count & 3 & 3 \\
\hline & truncated & $\%$ within raw material & $10.3 \%$ & $10.3 \%$ \\
\hline & rectangle & count & 1 & 1 \\
\hline & & \% within raw material & $3.4 \%$ & $3.4 \%$ \\
\hline & points & count & 9 & 9 \\
\hline & & $\%$ within raw material & $31.0 \%$ & $31.0 \%$ \\
\hline & & count & 29 & 29 \\
\hline Total & & $\%$ within raw material & $100.0 \%$ & $100.0 \%$ \\
\hline
\end{tabular}

Table 69. Hidasnémeti point types by raw material

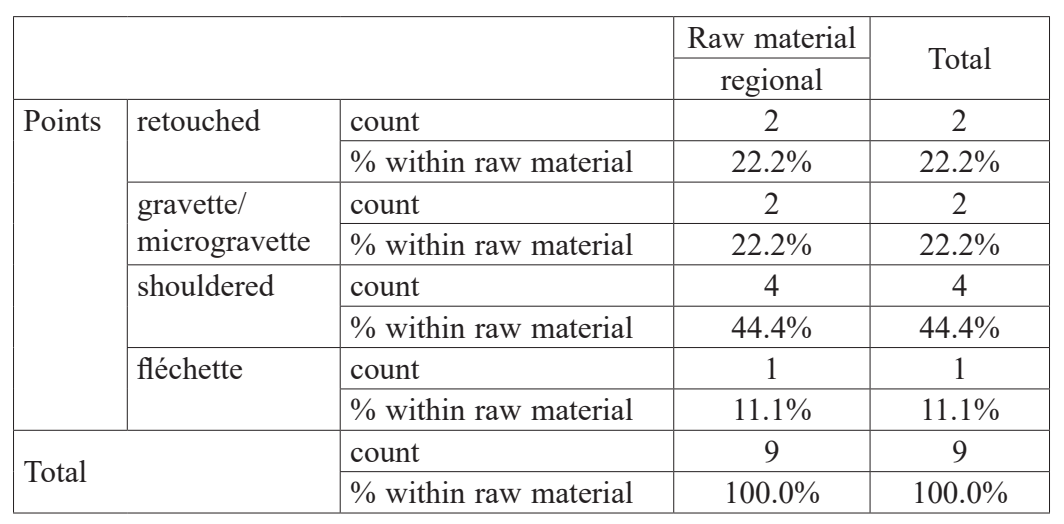


Table 70 . Hidasnémeti flake core types by raw material

\begin{tabular}{|l|l|l|c|c|}
\hline \multicolumn{2}{|c|}{} & Raw material & \multirow{2}{*}{ Total } \\
\cline { 3 - 5 } Types & unidirectional & count & 2 & 2 \\
\cline { 3 - 5 } & \% within raw material & $33.3 \%$ & $33.3 \%$ \\
\cline { 3 - 5 } & bidirectional & count & 1 & 1 \\
\cline { 3 - 5 } & multidirectional & \% within raw material & $16.7 \%$ & $16.7 \%$ \\
\cline { 3 - 5 } & & count & 3 & 3 \\
\hline \multirow{2}{*}{ Total } & count & $50.0 \%$ & $50.0 \%$ \\
\cline { 3 - 5 } & \% within raw material & 6 & 6 \\
\hline
\end{tabular}

Table 71. Hidasnémeti flake assemblage (complete specimens) dorsal scar pattern frequency by raw material

\begin{tabular}{|c|c|c|c|c|}
\hline & Raw material & \multirow{2}{*}{ Total } \\
\hline & & & regional & \\
\hline \multirow[t]{8}{*}{ Scars } & \multirow[t]{2}{*}{ unidirectional } & count & 83 & 83 \\
\hline & & $\%$ within raw material & $61.5 \%$ & $61.5 \%$ \\
\hline & \multirow[t]{2}{*}{ opposite } & count & 6 & 6 \\
\hline & & $\%$ within raw material & $4.4 \%$ & $4.4 \%$ \\
\hline & \multirow[t]{2}{*}{ perpendicular } & count & 37 & 37 \\
\hline & & $\%$ within raw material & $27.4 \%$ & $27.4 \%$ \\
\hline & \multirow[t]{2}{*}{ multiple } & count & 9 & 9 \\
\hline & & $\%$ within raw material & $6.7 \%$ & $6.7 \%$ \\
\hline \multirow{2}{*}{\multicolumn{2}{|c|}{ Total }} & count & 135 & 135 \\
\hline & & $\%$ within raw material & $100.0 \%$ & $100.0 \%$ \\
\hline
\end{tabular}

Table 72. Hidasnémeti flake assemblage (complete specimens) platform type frequency by raw material

\begin{tabular}{|c|c|c|c|c|}
\hline & Raw material & \multirow{2}{*}{ Total } \\
\hline & & & regional & \\
\hline \multirow[t]{8}{*}{ Platform } & \multirow[t]{2}{*}{ plain } & count & 97 & 97 \\
\hline & & $\%$ within raw material & $71.9 \%$ & $71.9 \%$ \\
\hline & \multirow[t]{2}{*}{ dihedral } & count & 15 & 15 \\
\hline & & $\%$ within raw material & $11.1 \%$ & $11.1 \%$ \\
\hline & \multirow[t]{2}{*}{ faceted } & count & 21 & 21 \\
\hline & & $\%$ within raw material & $15.6 \%$ & $15.6 \%$ \\
\hline & \multirow[t]{2}{*}{ cortical } & count & 2 & 2 \\
\hline & & $\%$ within raw material & $1.5 \%$ & $1.5 \%$ \\
\hline \multirow{2}{*}{\multicolumn{2}{|c|}{ Total }} & count & 135 & 135 \\
\hline & & $\%$ within raw material & $100.0 \%$ & $100.0 \%$ \\
\hline
\end{tabular}


Table 73. Hidasnémeti flake assemblage (complete specimens) impact point-overhang frequency by raw material

\begin{tabular}{|c|c|c|c|c|}
\hline & Raw material & \multirow{2}{*}{ Total } \\
\hline & & & regional & \\
\hline \multirow{8}{*}{$\begin{array}{l}\text { Impact } \\
\text { point-overhang }\end{array}$} & \multirow[t]{2}{*}{ none } & count & 51 & 51 \\
\hline & & $\%$ within raw material & $37.8 \%$ & $37.8 \%$ \\
\hline & \multirow[t]{2}{*}{ yes-no } & count & 12 & 12 \\
\hline & & $\%$ within raw material & $8.9 \%$ & $8.9 \%$ \\
\hline & \multirow[t]{2}{*}{ yes-yes } & count & 24 & 24 \\
\hline & & $\%$ within raw material & $17.8 \%$ & $17.8 \%$ \\
\hline & \multirow[t]{2}{*}{ no-yes } & count & 48 & 48 \\
\hline & & $\%$ within raw material & $35.6 \%$ & $35.6 \%$ \\
\hline \multirow{2}{*}{\multicolumn{2}{|c|}{ Total }} & count & 135 & 135 \\
\hline & & $\%$ within raw material & $100.0 \%$ & $100.0 \%$ \\
\hline
\end{tabular}

Table 74. Hidasnémeti flake blank assemblage length, width and thickness by raw materials

\begin{tabular}{|l|l|c|c|c|}
\hline \multicolumn{1}{|l|}{ Raw material } & Length [mm] & Width [mm] & Thickness [mm] \\
\hline Regional & Minimum & 13.09 & 14.57 & 2.15 \\
\cline { 2 - 5 } & Maximum & 69.82 & 92.45 & 23.80 \\
\cline { 2 - 5 } & Mean & 34.3324 & 31.4639 & 7.5630 \\
\cline { 2 - 5 } & Median & 32.9500 & 28.4400 & 6.5400 \\
\cline { 2 - 5 } & $\mathrm{N}$ & 135 & 135 & 135 \\
\cline { 2 - 5 } & Std. deviation & 11.91791 & 12.05520 & 3.80593 \\
\hline \multirow{5}{*}{ Total } & Minimum & 13.09 & 14.57 & 2.15 \\
\cline { 2 - 5 } & Maximum & 69.82 & 92.45 & 23.80 \\
\cline { 2 - 5 } & Mean & 34.3324 & 31.4639 & 7.5630 \\
\cline { 2 - 5 } & Median & 32.9500 & 28.4400 & 135 \\
\cline { 2 - 5 } & N & 135 & 135 & 3.80593 \\
\cline { 2 - 5 } & Std. deviation & 11.91791 & 12.05520 & \\
\hline
\end{tabular}

Table 75. Hidasnémeti flake tool length, width and thickness by raw materials

\begin{tabular}{|l|l|c|c|c|}
\hline \multicolumn{1}{|l|}{ Raw material } & Length [mm] & Width [mm] & Thickness [mm] \\
\hline Regional & Minimum & 18.24 & 9.15 & 3.06 \\
\cline { 2 - 5 } & Maximum & 128.59 & 82.87 & 30.10 \\
\cline { 2 - 5 } & Mean & 46.7668 & 38.0186 & 14.0623 \\
\cline { 2 - 5 } & Median & 42.4050 & 35.8800 & 13.3000 \\
\cline { 2 - 5 } & N & 22 & 22 & 22 \\
\cline { 2 - 5 } & Std. deviation & 26.22509 & 16.28582 & 6.56994 \\
\hline \multirow{5}{*}{ Total } & Minimum & 18.24 & 9.15 & 3.06 \\
\cline { 2 - 5 } & Maximum & 128.59 & 82.87 & 30.10 \\
\cline { 2 - 5 } & Mean & 46.7668 & 38.0186 & 14.0623 \\
\cline { 2 - 5 } & Median & 42.4050 & 35.8800 & 13.3000 \\
\cline { 2 - 5 } & N & 22 & 22 & 22 \\
\cline { 2 - 5 } & Std. deviation & 26.22509 & 16.28582 & 6.56994 \\
\hline
\end{tabular}


Table 76. Hidasnémeti flake blank and tool length, width and thickness mean t-test comparison by raw material

\begin{tabular}{|c|c|c|c|c|c|c|c|c|}
\hline \multicolumn{9}{|c|}{ Independent Samples Test } \\
\hline \multirow{2}{*}{\multicolumn{2}{|c|}{ Raw material }} & $\begin{array}{l}\text { Leven } \\
\text { for } \mathrm{E} \\
\text { of } \mathrm{Va}\end{array}$ & $\begin{array}{l}\text { Test } \\
\text { ality } \\
\text { inces }\end{array}$ & \multicolumn{5}{|c|}{ t-test for Equality of Means } \\
\hline & & $\mathrm{F}$ & Sig. & $\mathrm{t}$ & df & $\begin{array}{c}\text { Sig. } \\
\text { (2-tailed) }\end{array}$ & $\begin{array}{c}\text { Mean } \\
\text { difference }\end{array}$ & $\begin{array}{l}\text { Std. error } \\
\text { difference }\end{array}$ \\
\hline \multirow[t]{6}{*}{ Regional } & \multirow{2}{*}{$\begin{array}{l}\text { length } \\
{[\mathrm{mm}]}\end{array}$} & 13.051 & 0.000 & -3.680 & 155 & 0.000 & -12.43445 & 3.37887 \\
\hline & & & & -2.187 & 22.433 & 0.039 & -12.43445 & 5.68452 \\
\hline & \multirow{2}{*}{$\begin{array}{l}\text { width } \\
{[\mathrm{mm}]}\end{array}$} & 1.398 & 0.239 & -2.243 & 155 & 0.026 & -6.55471 & 2.92250 \\
\hline & & & & -1.809 & 24.887 & 0.083 & -6.55471 & 3.62385 \\
\hline & \multirow{2}{*}{$\begin{array}{l}\text { thickness } \\
{[\mathrm{mm}]}\end{array}$} & 13.677 & 0.000 & -6.595 & 155 & 0.000 & -6.49931 & 0.98545 \\
\hline & & & & -4.518 & 23.349 & 0.000 & -6.49931 & 1.43851 \\
\hline
\end{tabular}

Table 77. Hidasnémeti flake tool types by raw material

\begin{tabular}{|c|c|c|c|c|}
\hline & Raw material & \multirow{2}{*}{ Total } \\
\hline & & & regional & \\
\hline \multirow[t]{10}{*}{ Tooltypes } & \multirow[t]{2}{*}{ endscraper } & count & 4 & 4 \\
\hline & & $\%$ within raw material & $18.2 \%$ & $18.2 \%$ \\
\hline & \multirow[t]{2}{*}{ burin } & count & 4 & 4 \\
\hline & & $\%$ within raw material & $18.2 \%$ & $18.2 \%$ \\
\hline & \multirow[t]{2}{*}{ retouched } & count & 9 & 9 \\
\hline & & $\%$ within raw material & $40.9 \%$ & $40.9 \%$ \\
\hline & \multirow[t]{2}{*}{ borer } & count & 1 & 1 \\
\hline & & $\%$ within raw material & $4.5 \%$ & $4.5 \%$ \\
\hline & \multirow{2}{*}{$\begin{array}{l}\text { notched- } \\
\text { denticulated }\end{array}$} & count & 4 & 4 \\
\hline & & $\%$ within raw material & $18.2 \%$ & $18.2 \%$ \\
\hline \multirow{2}{*}{\multicolumn{2}{|c|}{ Total }} & count & 22 & 22 \\
\hline & & $\%$ within raw material & $100.0 \%$ & $100.0 \%$ \\
\hline
\end{tabular}

Table 78. Hidasnémeti complete tool assemblage by raw material

\begin{tabular}{|c|c|c|c|c|c|}
\hline & \multicolumn{2}{|c|}{ Raw material } & \multirow{2}{*}{ Total } \\
\hline & & & regional & transcarpathian & \\
\hline \multirow{16}{*}{ Tooltypes } & \multirow[t]{2}{*}{ endscraper } & count & 18 & 0 & 18 \\
\hline & & $\%$ within raw material & $14.1 \%$ & $0.0 \%$ & $13.8 \%$ \\
\hline & \multirow[t]{2}{*}{ burin } & count & 43 & 1 & 44 \\
\hline & & $\%$ within raw material & $33.6 \%$ & $50.0 \%$ & $33.8 \%$ \\
\hline & \multirow[t]{2}{*}{ retouched } & count & 26 & 0 & 26 \\
\hline & & $\%$ within raw material & $20.3 \%$ & $0.0 \%$ & $20.0 \%$ \\
\hline & \multirow[t]{2}{*}{ borer } & count & 1 & 0 & 1 \\
\hline & & $\%$ within raw material & $0.8 \%$ & $0.0 \%$ & $0.8 \%$ \\
\hline & \multirow{2}{*}{ truncation } & count & 4 & 0 & 4 \\
\hline & & $\%$ within raw material & $3.1 \%$ & $0.0 \%$ & $3.1 \%$ \\
\hline & \multirow{2}{*}{$\begin{array}{l}\text { notched- } \\
\text { denticulate }\end{array}$} & count & 7 & 0 & 7 \\
\hline & & $\%$ within raw material & $5.5 \%$ & $0.0 \%$ & $5.4 \%$ \\
\hline & \multirow[t]{2}{*}{ composite } & count & 0 & 1 & 1 \\
\hline & & $\%$ within raw material & $0.0 \%$ & $50.0 \%$ & $0.8 \%$ \\
\hline & \multirow[t]{2}{*}{ armature } & count & 29 & 0 & 29 \\
\hline & & $\%$ within raw material & $22.7 \%$ & $0.0 \%$ & $22.3 \%$ \\
\hline \multirow{2}{*}{\multicolumn{2}{|c|}{ Total }} & count & 128 & 2 & 130 \\
\hline & & $\%$ within raw material & $100.0 \%$ & $100.0 \%$ & $100.0 \%$ \\
\hline
\end{tabular}




\section{SAJÓSZENTPÉTER}

The site

The site is found $150 \mathrm{~m}$ a.s.l. in the Sajó valley the northeast Hungary, on the eastern edge of Bükk mountains, on a slope facing east. Excavations were carried out at the site between 1990 and 1992 (RINGER, Holló 2001). The stratigraphy consisted of mostly eolian sediments of six meters under the recent top soil. Layer 2 of unspecified thickness yielded most of the archaeological finds but artifacts were found sporadically in layers 1, 5, 6, and 12. Layer 2 was identified as forest-steppe soil level A developed at the end of MIS3. RiNGER and Holló (2001) identified the Gravettian occupation in layer 2 Pavlovian, while in layer 5 carinated end scrapers were found which most probably belonged to an Aurignacian occupation. The material studied here includes the artifacts of layer 2.

\section{Raw materials}

The majority of the raw materials is limnic silicite of two types: a white and the dark colored. Of these two, the white is dominant. The white was earlier identified as a local material (RINGER, Holló 2001). However, no such outcrop is known within $10 \mathrm{~km}$ area of the site. This most probably derived from the southern Bükk Mountains, from where the white variant was mentioned (BIRó, DoвOsI 1991). The dark variant could have derived from the Zemplén. Further materials are the meta-rhyolite of Bükk mountains, the radiolarite, and a single piece of Świeciechów flint from Poland. Except the latter, all the lithic raw materials are of regional origin, which yielded 79.2 item per kg (Tab. 79).

\section{Blade tool prodcution}

The complete sequence of lithic production was found in the assemblage (Tab. 80). The sole piece of transcarpathian material does not seem to have been produced at the site.

Blade debitage was accomplished applying unidirectional debitage (Tab. 81), however, single platform blade cores only rule the assemblage if pre-cores are included, otherwise, cores with more than one platform are abundant (Tab. 82). The blade platforms are plain (Tab. 83), and soft hammer technique ruled the knapping (Tab. 84). The mean length of the blades is $47.4 \mathrm{~mm}$ (Tab. 85).

Blades are prevalent in the tool kit (Tab. 86). Also, over $20 \%$ of the blades are tools (Tab. 87). The mean size of the blade tools are similar to the blanks (Tab. 88) and the t-test showed there is no difference between blank and tool blades (Tab. 89).

Most blade tools are burins and then armatures (Tab. 90). The armatures are generally points (Tab. 91), especially the Gravette type beside the only Vachons point (Tab. 92). 


\section{Flake tool production}

Flakes did not have a debitage and their production completely could be the byproduct of the blade debitage. Flakes have mostly unidirectional scars (Tab. 93), their platforms are typically plain (Tab. 94), and the frequency of impact point and unabraded overhang show frequent soft hammer percussion (Tab. 95).

The flakes are small (Tab. 96) but the tools (Tab. 97) are greater in length and thickness (Tab. 98). Only two types of tools were made of flakes: burin and end scraper (Tab. 99).

Table 79. Sajószentpéter lithic raw material composition by weight in grams

\begin{tabular}{|l|c|c|c|c|c|c|}
\hline Raw material & Blade & Flake & Debris & Core & Total & $\%$ \\
\hline Regional & 770.92 & 852.57 & 597.06 & 1845.06 & 4065.61 & 99.78402 \\
\hline Within regional \% & 18.96198 & 20.97028 & 14.68562 & 45.38212 & 100 & \\
\hline Transarpathian & & 8.8 & & & 8.8 & 0.215982 \\
\hline Within transcarpathian \% & & 100 & & & 100 & \\
\hline Total & 770.92 & 861.37 & 597.06 & 1845.06 & 4074.41 & 100 \\
\hline$\%$ & 18.92102 & 21.14098 & 14.6539 & 45.2841 & 100 & \\
\hline
\end{tabular}

Table 80 . Sajószentpéter lithic assemblage composition by raw material types and technological categories

\begin{tabular}{|c|c|c|c|c|c|}
\hline & \multicolumn{2}{|c|}{ Raw material } & \multirow{2}{*}{ Total } \\
\hline & & & regional & transcarpathian & \\
\hline \multirow[t]{14}{*}{ Class } & \multirow[t]{2}{*}{ flake } & count & 82 & 1 & 83 \\
\hline & & \% within raw material & $25.5 \%$ & $100.0 \%$ & $25.7 \%$ \\
\hline & \multirow[t]{2}{*}{ blade } & count & 108 & 0 & 108 \\
\hline & & $\%$ within raw material & $33.5 \%$ & $0.0 \%$ & $33.4 \%$ \\
\hline & \multirow[t]{2}{*}{ debris } & count & 95 & 0 & 95 \\
\hline & & $\%$ within raw material & $29.5 \%$ & $0.0 \%$ & $29.4 \%$ \\
\hline & \multirow{2}{*}{$\begin{array}{l}\text { rejuvenating } \\
\text { flake }\end{array}$} & count & 11 & 0 & 11 \\
\hline & & $\%$ within raw material & $3.4 \%$ & $0.0 \%$ & $3.4 \%$ \\
\hline & \multirow[t]{2}{*}{ crest } & count & 8 & 0 & 8 \\
\hline & & $\%$ within raw material & $2.5 \%$ & $0.0 \%$ & $2.5 \%$ \\
\hline & \multirow[t]{2}{*}{ neo-crest } & count & 8 & 0 & 8 \\
\hline & & \% within raw material & $2.5 \%$ & $0.0 \%$ & $2.5 \%$ \\
\hline & \multirow[t]{2}{*}{ blade core } & count & 10 & 0 & 10 \\
\hline & & $\%$ within raw material & $3.1 \%$ & $0.0 \%$ & $3.1 \%$ \\
\hline \multirow{2}{*}{\multicolumn{2}{|c|}{ Total }} & count & 322 & 1 & 323 \\
\hline & & $\%$ within raw material & $100.0 \%$ & $100.0 \%$ & $100.0 \%$ \\
\hline
\end{tabular}


Lithic analysis of the Middle and Late Upper Palaeolithic in Hungary

Table 81. Sajószentpéter blade assemblage (complete specimens) dorsal scar pattern frequency by raw material

\begin{tabular}{|c|c|c|c|c|}
\hline & Raw material & \multirow{2}{*}{ Total } \\
\hline & & & regional & \\
\hline \multirow[t]{10}{*}{ Scars } & \multirow[t]{2}{*}{ unidirectional } & count & 15 & 15 \\
\hline & & $\%$ within raw material & $60.0 \%$ & $60.0 \%$ \\
\hline & \multirow[t]{2}{*}{ opposite } & count & 3 & 3 \\
\hline & & $\%$ within raw material & $12.0 \%$ & $12.0 \%$ \\
\hline & \multirow[t]{2}{*}{ perpendicular } & count & 5 & 5 \\
\hline & & $\%$ within raw material & $20.0 \%$ & $20.0 \%$ \\
\hline & \multirow[t]{2}{*}{ multiple } & count & 1 & 1 \\
\hline & & $\%$ within raw material & $4.0 \%$ & $4.0 \%$ \\
\hline & \multirow[t]{2}{*}{ no scar } & count & 1 & 1 \\
\hline & & $\%$ within raw material & $4.0 \%$ & $4.0 \%$ \\
\hline \multirow{2}{*}{\multicolumn{2}{|c|}{ Total }} & count & 25 & 25 \\
\hline & & $\%$ within raw material & $100.0 \%$ & $100.0 \%$ \\
\hline
\end{tabular}

Table 82 . Sajószentpéter blade core types by raw material

\begin{tabular}{|c|c|c|c|c|}
\hline & Raw material & \multirow{2}{*}{ Total } \\
\hline & & & regional & \\
\hline \multirow[t]{8}{*}{ Types } & \multirow[t]{2}{*}{ unidirectional } & count & 3 & 3 \\
\hline & & $\%$ within raw material & $30.0 \%$ & $30.0 \%$ \\
\hline & \multirow[t]{2}{*}{ bidirectional } & count & 3 & 3 \\
\hline & & $\%$ within raw material & $30.0 \%$ & $30.0 \%$ \\
\hline & \multirow[t]{2}{*}{ multidirectional } & count & 1 & 1 \\
\hline & & $\%$ within raw material & $10.0 \%$ & $10.0 \%$ \\
\hline & \multirow[t]{2}{*}{ pre-core } & count & 3 & 3 \\
\hline & & $\%$ within raw material & $30.0 \%$ & $30.0 \%$ \\
\hline \multirow{2}{*}{\multicolumn{2}{|c|}{ Total }} & count & 10 & 10 \\
\hline & & $\%$ within raw material & $100.0 \%$ & $100.0 \%$ \\
\hline
\end{tabular}

Table 83. Sajószentpéter blade assemblage (complete specimens) platform type frequency by raw material

\begin{tabular}{|c|c|c|c|c|}
\hline & Raw material & \multirow{2}{*}{ Total } \\
\hline & & & regional & \\
\hline \multirow[t]{10}{*}{ Platform } & \multirow[t]{2}{*}{ plain } & count & 15 & 15 \\
\hline & & $\%$ within raw material & $60.0 \%$ & $60.0 \%$ \\
\hline & \multirow[t]{2}{*}{ faceted } & count & 5 & 5 \\
\hline & & $\%$ within raw material & $20.0 \%$ & $20.0 \%$ \\
\hline & \multirow[t]{2}{*}{ cortical } & count & 2 & 2 \\
\hline & & $\%$ within raw material & $8.0 \%$ & $8.0 \%$ \\
\hline & \multirow[t]{2}{*}{ linear } & count & 2 & 2 \\
\hline & & $\%$ within raw material & $8.0 \%$ & $8.0 \%$ \\
\hline & \multirow[t]{2}{*}{ damaged } & count & 1 & 1 \\
\hline & & $\%$ within raw material & $4.0 \%$ & $4.0 \%$ \\
\hline \multirow{2}{*}{\multicolumn{2}{|c|}{ Total }} & count & 25 & 25 \\
\hline & & $\%$ within raw material & $100.0 \%$ & $100.0 \%$ \\
\hline
\end{tabular}


Table 84. Sajószentpéter blade assemblage (complete specimens) impact point-overhang co-presence by raw material

\begin{tabular}{|c|c|c|c|c|}
\hline & Raw material & \multirow{2}{*}{ Total } \\
\hline & & & regional & \\
\hline \multirow{8}{*}{$\begin{array}{l}\text { Impact } \\
\text { point-overhang }\end{array}$} & \multirow[t]{2}{*}{ none } & count & 21 & 21 \\
\hline & & $\%$ within raw material & $84.0 \%$ & $84.0 \%$ \\
\hline & \multirow[t]{2}{*}{ yes-no } & count & 1 & 1 \\
\hline & & $\%$ within raw material & $4.0 \%$ & $4.0 \%$ \\
\hline & \multirow[t]{2}{*}{ yes-yes } & count & 1 & 1 \\
\hline & & $\%$ within raw material & $4.0 \%$ & $4.0 \%$ \\
\hline & \multirow[t]{2}{*}{ no-yes } & count & 2 & 2 \\
\hline & & $\%$ within raw material & $8.0 \%$ & $8.0 \%$ \\
\hline \multirow{2}{*}{\multicolumn{2}{|c|}{ Total }} & count & 25 & 25 \\
\hline & & $\%$ within raw material & $100.0 \%$ & $100.0 \%$ \\
\hline
\end{tabular}

Table 85. Sajószentpéter blade assemblage (complete specimens) length, width and thickness by raw material

\begin{tabular}{|l|l|c|c|c|}
\hline \multicolumn{2}{|l|}{ Raw material } & Length [mm] & Width [mm] & Thickness [mm] \\
\hline \multirow{4}{*}{ Regional } & Minimum & 19.98 & 9.37 & 1.98 \\
\cline { 2 - 5 } & Maximum & 97.55 & 31.97 & 17.85 \\
\cline { 2 - 5 } & Mean & 47.3896 & 17.6184 & 6.6948 \\
\cline { 2 - 5 } & Median & 45.0900 & 16.1400 & 5.5100 \\
\cline { 2 - 5 } & N & 25 & 25 & 25 \\
\cline { 2 - 5 } & Std. deviation & 18.10467 & 6.41317 & 3.31909 \\
\hline
\end{tabular}

Table 86. Sajószentpéter tool assemblage product composition by raw materials

\begin{tabular}{|c|c|c|c|c|}
\hline & \multicolumn{2}{|c|}{ Raw material } & \multirow{2}{*}{ Total } \\
\hline & & regional & transcarpathian & \\
\hline \multirow[t]{2}{*}{ Flake } & count & 15 & 1 & 16 \\
\hline & $\%$ within raw material & $34.1 \%$ & $100.0 \%$ & $35.6 \%$ \\
\hline \multirow[t]{2}{*}{ Blade } & count & 27 & 0 & 27 \\
\hline & $\%$ within raw material & $61.4 \%$ & $0.0 \%$ & $60.0 \%$ \\
\hline \multirow[t]{2}{*}{ Debris } & count & 2 & 0 & 2 \\
\hline & $\%$ within raw material & $4.5 \%$ & $0.0 \%$ & $4.4 \%$ \\
\hline \multirow{2}{*}{ Total } & count & 44 & 1 & 45 \\
\hline & $\%$ within raw material & $100.0 \%$ & $100.0 \%$ & $100.0 \%$ \\
\hline
\end{tabular}


Lithic analysis of the Middle and Late Upper Palaeolithic in Hungary

Table 87. Sajószentpéter knapped product frequency in tool assemblage by raw materials

\begin{tabular}{|c|c|c|c|c|c|c|}
\hline & \multicolumn{2}{|c|}{ Raw material } & \multirow{2}{*}{ Total } \\
\hline & & & & regional & transcarpathian & \\
\hline \multirow[t]{6}{*}{ Flake } & \multirow[t]{4}{*}{ state } & \multirow[t]{2}{*}{ blank } & count & 67 & 0 & 67 \\
\hline & & & $\%$ within raw material & $81.7 \%$ & $0.0 \%$ & $80.7 \%$ \\
\hline & & \multirow[t]{2}{*}{ tool } & count & 15 & 1 & 16 \\
\hline & & & $\%$ within raw material & $18.3 \%$ & $100.0 \%$ & $19.3 \%$ \\
\hline & \multirow{2}{*}{\multicolumn{2}{|c|}{ total }} & count & 82 & 1 & 83 \\
\hline & & & $\%$ within raw material & $100.0 \%$ & $100.0 \%$ & $100.0 \%$ \\
\hline \multirow[t]{6}{*}{ Blade } & \multirow[t]{4}{*}{ state } & \multirow[t]{2}{*}{ blank } & count & 97 & & 97 \\
\hline & & & $\%$ within raw material & $78.2 \%$ & & $78.2 \%$ \\
\hline & & \multirow[t]{2}{*}{ tool } & count & 27 & & 27 \\
\hline & & & $\%$ within raw material & $21.8 \%$ & & $21.8 \%$ \\
\hline & \multirow{2}{*}{\multicolumn{2}{|c|}{ total }} & count & 124 & & 124 \\
\hline & & & $\%$ within raw material & $100.0 \%$ & & $100.0 \%$ \\
\hline \multirow[t]{6}{*}{ Debris } & \multirow[t]{4}{*}{ state } & \multirow[t]{2}{*}{ blank } & count & 93 & & 93 \\
\hline & & & $\%$ within raw material & $97.9 \%$ & & $97.9 \%$ \\
\hline & & \multirow[t]{2}{*}{ tool } & count & 2 & & 2 \\
\hline & & & $\%$ within raw material & $2.1 \%$ & & $2.1 \%$ \\
\hline & \multirow{2}{*}{\multicolumn{2}{|c|}{ total }} & count & 95 & & 95 \\
\hline & & & $\%$ within raw material & $100.0 \%$ & & $100.0 \%$ \\
\hline
\end{tabular}

Table 88. Sajószentpéter blade tool assemblage length, width and thickness by raw materials

\begin{tabular}{|l|l|c|c|c|}
\hline \multicolumn{2}{|l|}{ Raw material } & Length [mm] & Width [mm] & Thickness [mm] \\
\hline \multirow{4}{*}{ Regional } & Minimum & 15.89 & 5.91 & 2.83 \\
\cline { 2 - 5 } & Maximum & 92.04 & 41.90 & 16.71 \\
\cline { 2 - 5 } & Mean & 48.8670 & 19.0722 & 8.4748 \\
\cline { 2 - 5 } & Median & 49.2500 & 18.8400 & 6.9400 \\
\cline { 2 - 5 } & N & 27 & 27 & 27 \\
\cline { 2 - 5 } & Std. deviation & 21.07666 & 9.07632 & 4.53766 \\
\hline
\end{tabular}

Table 89. Sajószentpéter blade blank and tool length, width and thickness mean t-test comparison by raw material

\begin{tabular}{|c|c|c|c|c|c|c|c|c|}
\hline \multicolumn{9}{|c|}{ Independent Samples Test } \\
\hline \multirow{2}{*}{\multicolumn{2}{|c|}{ Raw material }} & $\begin{array}{l}\text { Lever } \\
\text { for } \mathrm{E} \\
\text { of } \mathrm{Va}\end{array}$ & $\begin{array}{l}\text { s Test } \\
\text { uality } \\
\text { ances }\end{array}$ & \multicolumn{5}{|c|}{ t-test for Equality of Means } \\
\hline & & $\mathrm{F}$ & Sig. & $\mathrm{t}$ & $\mathrm{df}$ & $\begin{array}{c}\text { Sig. } \\
\text { (2-tailed) }\end{array}$ & $\begin{array}{c}\text { Mean } \\
\text { difference }\end{array}$ & $\begin{array}{l}\text { Std. error } \\
\text { difference }\end{array}$ \\
\hline \multirow[t]{6}{*}{ Regional } & \multirow{2}{*}{$\begin{array}{l}\text { length } \\
{[\mathrm{mm}]}\end{array}$} & 1.031 & 0.315 & -0.270 & 50 & 0.788 & -1.47744 & 5.46954 \\
\hline & & & & -0.272 & 49.734 & 0.787 & -1.47744 & 5.43728 \\
\hline & \multirow{2}{*}{$\begin{array}{l}\text { width } \\
\text { [mm] }\end{array}$} & 1.291 & 0.261 & -0.662 & 50 & 0.511 & -1.45382 & 2.19566 \\
\hline & & & & -0.671 & 46.844 & 0.506 & -1.45382 & 2.16708 \\
\hline & \multirow{2}{*}{$\begin{array}{l}\text { thickness } \\
{[\mathrm{mm}]}\end{array}$} & 6.472 & 0.014 & -1.604 & 50 & 0.115 & -1.78001 & 1.11004 \\
\hline & & & & -1.623 & 47.534 & 0.111 & -1.78001 & 1.09693 \\
\hline
\end{tabular}


Table 90. Sajószentpéter blade tool types by raw material

\begin{tabular}{|c|c|c|c|c|}
\hline & Raw material & \multirow{2}{*}{ Total } \\
\hline & & & regional & \\
\hline \multirow[t]{16}{*}{ Tooltypes } & \multirow[t]{2}{*}{ endscraper } & count & 3 & 3 \\
\hline & & $\%$ within raw material & $11.1 \%$ & $11.1 \%$ \\
\hline & \multirow[t]{2}{*}{ burin } & count & 9 & 9 \\
\hline & & $\%$ within raw material & $33.3 \%$ & $33.3 \%$ \\
\hline & \multirow[t]{2}{*}{ retouched } & count & 5 & 5 \\
\hline & & $\%$ within raw material & $18.5 \%$ & $18.5 \%$ \\
\hline & \multirow[t]{2}{*}{ borer } & count & 1 & 1 \\
\hline & & $\%$ within raw material & $3.7 \%$ & $3.7 \%$ \\
\hline & \multirow[t]{2}{*}{ armature } & count & 5 & 5 \\
\hline & & $\%$ within raw material & $18.5 \%$ & $18.5 \%$ \\
\hline & \multirow[t]{2}{*}{ truncation } & count & 1 & 1 \\
\hline & & $\%$ within raw material & $3.7 \%$ & $3.7 \%$ \\
\hline & \multirow[t]{2}{*}{ composite } & count & 1 & 1 \\
\hline & & $\%$ within raw material & $3.7 \%$ & $3.7 \%$ \\
\hline & \multirow{2}{*}{$\begin{array}{l}\text { notched- } \\
\text { denticulated }\end{array}$} & count & 2 & 2 \\
\hline & & $\%$ within raw material & $7.4 \%$ & $7.4 \%$ \\
\hline \multirow{2}{*}{\multicolumn{2}{|c|}{ Total }} & count & 27 & 27 \\
\hline & & $\%$ within raw material & $100.0 \%$ & $100.0 \%$ \\
\hline
\end{tabular}

Table 91. Sajószentpéter armature types by raw material

\begin{tabular}{|l|l|l|c|c|}
\hline \multicolumn{2}{|c|}{} & Raw material & \multirow{2}{*}{ Total } \\
\cline { 3 - 4 } Armature & \multirow{2}{*}{ backed } & count & 1 & 1 \\
\cline { 3 - 5 } & & $\%$ within raw material & $20.0 \%$ & $20.0 \%$ \\
\cline { 3 - 5 } & \multirow{2}{*}{ point } & count & 4 & 4 \\
\cline { 3 - 5 } & \% within raw material & $80.0 \%$ & $80.0 \%$ \\
\hline \multirow{2}{*}{ Total } & count & 5 & 5 \\
\cline { 3 - 5 } & \% within raw material & $100.0 \%$ & $100.0 \%$ \\
\hline
\end{tabular}

Table 92. Sajószentpéter point types by raw material

\begin{tabular}{|c|c|c|c|c|}
\hline & Raw material & \multirow{2}{*}{ Total } \\
\hline & & & regional & \\
\hline \multirow[t]{4}{*}{ Point } & \multirow[t]{2}{*}{ grav } & count & 3 & 3 \\
\hline & & \% within raw material & $75.0 \%$ & $75.0 \%$ \\
\hline & \multirow[t]{2}{*}{ vachons } & count & 1 & 1 \\
\hline & & $\%$ within raw material & $25.0 \%$ & $25.0 \%$ \\
\hline \multirow{2}{*}{\multicolumn{2}{|c|}{ Total }} & count & 4 & 4 \\
\hline & & $\%$ within raw material & $100.0 \%$ & $100.0 \%$ \\
\hline
\end{tabular}


Table 93. Sajószentpéter flake assemblage (complete specimens) dorsal scar pattern frequency by raw material

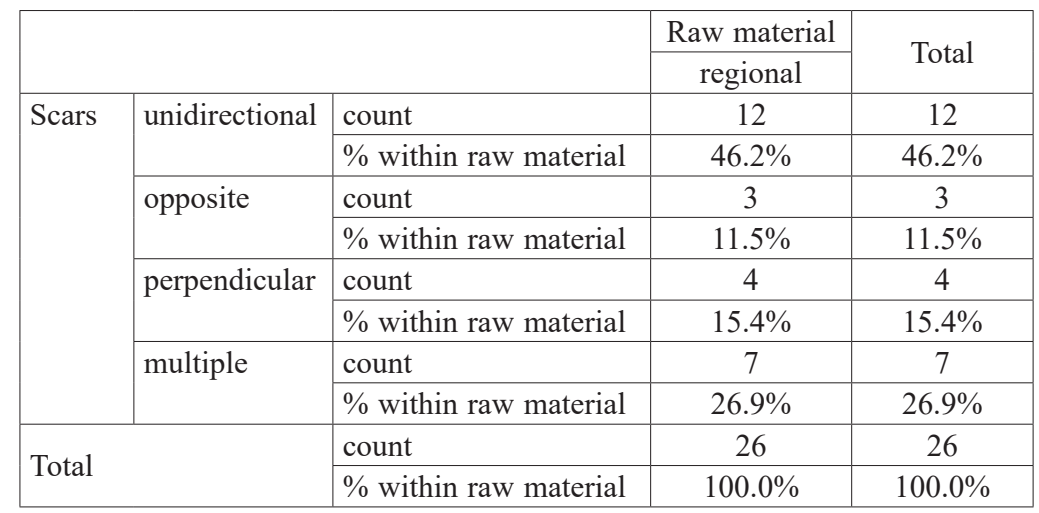

Table 94. Sajószentpéter flake assemblage (complete specimens) platform type frequency by raw material

\begin{tabular}{|c|c|c|c|c|}
\hline & Raw material & \multirow{2}{*}{ Total } \\
\hline & & & regional & \\
\hline \multirow[t]{8}{*}{ Platform } & \multirow[t]{2}{*}{ plain } & count & 19 & 19 \\
\hline & & \% within raw material & $73.1 \%$ & $73.1 \%$ \\
\hline & \multirow[t]{2}{*}{ dihedral } & count & 3 & 3 \\
\hline & & $\%$ within raw material & $11.5 \%$ & $11.5 \%$ \\
\hline & \multirow[t]{2}{*}{ faceted } & count & 3 & 3 \\
\hline & & \% within raw material & $11.5 \%$ & $11.5 \%$ \\
\hline & \multirow[t]{2}{*}{ punctiform } & count & 1 & 1 \\
\hline & & $\%$ within raw material & $3.8 \%$ & $3.8 \%$ \\
\hline \multirow{2}{*}{\multicolumn{2}{|c|}{ Total }} & count & 26 & 26 \\
\hline & & $\%$ within raw material & $100.0 \%$ & $100.0 \%$ \\
\hline
\end{tabular}

Table 95. Sajószentpéter flake assemblage (complete specimens) impact point-overhang presence frequency by raw material

\begin{tabular}{|c|c|c|c|c|}
\hline & Raw material & \multirow{2}{*}{ Total } \\
\hline & & & regional & \\
\hline \multirow{8}{*}{$\begin{array}{l}\text { Impact } \\
\text { point-overhang }\end{array}$} & \multirow[t]{2}{*}{ none } & count & 10 & 10 \\
\hline & & $\%$ within raw material & $38.5 \%$ & $38.5 \%$ \\
\hline & \multirow[t]{2}{*}{ yes-no } & count & 2 & 2 \\
\hline & & $\%$ within raw material & $7.7 \%$ & $7.7 \%$ \\
\hline & \multirow[t]{2}{*}{ yes-yes } & count & 6 & 6 \\
\hline & & $\%$ within raw material & $23.1 \%$ & $23.1 \%$ \\
\hline & \multirow[t]{2}{*}{ no-yes } & count & 8 & 8 \\
\hline & & $\%$ within raw material & $30.8 \%$ & $30.8 \%$ \\
\hline \multirow{2}{*}{\multicolumn{2}{|c|}{ Total }} & count & 26 & 26 \\
\hline & & \% within raw material & $100.0 \%$ & $100.0 \%$ \\
\hline
\end{tabular}


Table 96. Sajószentpéter flake assemblage length, width and thickness by raw materials

\begin{tabular}{|l|l|c|c|c|}
\hline \multicolumn{1}{|l|}{ Raw material } & Length [mm] & Width [mm] & Thickness [mm] \\
\hline \multirow{5}{*}{ Regional } & Minimum & 12.41 & 12.49 & 2.42 \\
\cline { 2 - 5 } & Maximum & 67.44 & 58.97 & 21.18 \\
\cline { 2 - 5 } & Mean & 33.7927 & 31.2938 & 8.4531 \\
\cline { 2 - 5 } & Median & 30.9950 & 28.0050 & 7.7400 \\
\cline { 2 - 5 } & $\mathrm{N}$ & 26 & 26 & 26 \\
\cline { 2 - 5 } & Std. deviation & 13.17213 & 13.70176 & 4.42157 \\
\hline \multirow{5}{*}{ Total } & Minimum & 12.41 & 12.49 & 2.42 \\
\cline { 2 - 5 } & Maximum & 67.44 & 58.97 & 8.4531 \\
\cline { 2 - 5 } & Mean & 33.7927 & 31.2938 & 7.7400 \\
\cline { 2 - 5 } & Median & 30.9950 & 28.0050 & 26 \\
\cline { 2 - 5 } & N & 26 & 26 & 4.42157 \\
\cline { 2 - 5 } & Std. deviation & 13.17213 & 13.70176 & \\
\hline
\end{tabular}

Table 97. Sajószentpéter flake tool assemblage length, width and thickness by raw materials

\begin{tabular}{|c|c|c|c|c|}
\hline \multicolumn{2}{|l|}{ Raw material } & Length [mm] & Width [mm] & Thickness [mm] \\
\hline \multirow[t]{6}{*}{ Regional } & Minimum & 27.59 & 12.49 & 4.40 \\
\hline & Maximum & 77.08 & 52.54 & 22.89 \\
\hline & Mean & 44.0473 & 30.0647 & 11.9013 \\
\hline & Median & 42.4000 & 27.8200 & 10.6400 \\
\hline & $\mathrm{N}$ & 15 & 15 & 15 \\
\hline & Std. deviation & 12.41200 & 11.55739 & 5.23638 \\
\hline \multirow[t]{6}{*}{ Transcarpathian } & Minimum & 42.40 & 21.69 & 10.92 \\
\hline & Maximum & 42.40 & 21.69 & 10.92 \\
\hline & Mean & 42.4000 & 21.6900 & 10.9200 \\
\hline & Median & 42.4000 & 21.6900 & 10.9200 \\
\hline & $\mathrm{N}$ & 1 & 1 & 1 \\
\hline & Std. deviation & - & - & - \\
\hline \multirow[t]{6}{*}{ Total } & Minimum & 27.59 & 12.49 & 4.40 \\
\hline & Maximum & 77.08 & 52.54 & 22.89 \\
\hline & Mean & 43.9444 & 29.5413 & 11.8400 \\
\hline & Median & 42.4000 & 26.6600 & 10.7800 \\
\hline & $\mathrm{N}$ & 16 & 16 & 16 \\
\hline & Std. deviation & 11.99820 & 11.36010 & 5.06476 \\
\hline
\end{tabular}

Table 98. Sajószentpéter flake blank and tool length, width and thickness mean t-test comparison

\begin{tabular}{|c|c|c|c|c|c|c|c|c|}
\hline \multicolumn{9}{|c|}{ Independent Samples Test } \\
\hline & & \multicolumn{2}{|c|}{$\begin{array}{c}\text { Levene's Test } \\
\text { for Equality } \\
\text { of Variances }\end{array}$} & \multicolumn{5}{|c|}{ t-test for Equality of Means } \\
\hline & & $\mathrm{F}$ & Sig. & $\mathrm{t}$ & df & $\begin{array}{c}\text { Sig. } \\
\text { (2-tailed) }\end{array}$ & $\begin{array}{c}\text { Mean } \\
\text { difference }\end{array}$ & $\begin{array}{l}\text { Std. error } \\
\text { difference }\end{array}$ \\
\hline \multirow[t]{6}{*}{ Flake } & \multirow{2}{*}{$\begin{array}{l}\text { length } \\
{[\mathrm{mm}]}\end{array}$} & 0.563 & 0.458 & -2.507 & 40 & 0.016 & -10.15168 & 4.04952 \\
\hline & & & & -2.564 & 34.211 & 0.015 & -10.15168 & 3.95861 \\
\hline & \multirow{2}{*}{$\begin{array}{l}\text { width } \\
{[\mathrm{mm}]}\end{array}$} & 0.803 & 0.376 & 0.428 & 40 & 0.671 & 1.75260 & 4.09053 \\
\hline & & & & 0.448 & 36.383 & 0.657 & 1.75260 & 3.90979 \\
\hline & \multirow{2}{*}{$\begin{array}{l}\text { thickness } \\
{[\mathrm{mm}]}\end{array}$} & 0.863 & 0.358 & -2.281 & 40 & 0.028 & -3.38692 & 1.48487 \\
\hline & & & & -2.207 & 28.596 & 0.036 & -3.38692 & 1.53466 \\
\hline
\end{tabular}


Table 99. Sajószentpéter flake tool types by raw material

\begin{tabular}{|c|c|c|c|c|c|}
\hline & \multicolumn{2}{|c|}{ Raw material } & \multirow{2}{*}{ Total } \\
\hline & & & regional & transcarpathian & \\
\hline \multirow[t]{4}{*}{ Tooltypes } & \multirow[t]{2}{*}{ endscraper } & count & 1 & 0 & 1 \\
\hline & & $\%$ within raw material & $6.7 \%$ & $0.0 \%$ & $6.3 \%$ \\
\hline & \multirow[t]{2}{*}{ burin } & count & 14 & 1 & 15 \\
\hline & & $\%$ within raw material & $93.3 \%$ & $100.0 \%$ & $93.8 \%$ \\
\hline \multirow{2}{*}{\multicolumn{2}{|c|}{ Total }} & count & 15 & 1 & 16 \\
\hline & & \% within raw material & $100.0 \%$ & $100.0 \%$ & $100.0 \%$ \\
\hline
\end{tabular}

\section{SÁGVÁR}

\section{The site}

Village Ságvár is located about $10 \mathrm{~km}$ south of Lake Balaton. The Palaeolithic site is situated outside of the village's core, in the hilly area of this region, on a hill (Lyukas-domb) rising $228 \mathrm{~m}$ a.s.l. above creek Jaba.

Several excavations were conducted at the site between 1928 and 1959 (LENGYEL 2010). The site preserved two archaeological layers imbedded in loess. According to GÁBORI and GÁBORI-CsÁNK (1957) two dark organic material rich bands represented the layers. In trench I of GÁBORI's excavation (GÁBORI 1959) the upper archaeological level appeared $1.2 \mathrm{~m}$ below the topsoil surface and its thickness was $8-10 \mathrm{~cm}$. Its general color was gray with blackish-brown hue, and it consisted of small red granules, bone morsels, and ash. The layer included hearths, which had been remarkable features of the site since the 1930s.

Also, a hut basement with post holes that abundantly contained bones, antlers, and knapped lithics was recovered (GÁBORI 1965). The lower archaeological layer lay $1.5 \mathrm{~m}$ beneath the upper level, separated by archaeologically sterile loess. Expanding over a maximum 2 square meters area, it contained seven hearths.

The fauna consisted of reindeer, horse and the mammoth (VöRös 1982). A perforated shell of Arca diluvia and pieces of red ochre were also found.

Microscopic analysis of charred wood pieces 1 to $5 \mathrm{~cm}$ large from the hearths excavated in the 1930s identified pine tree tissue (LaczKó et al. 1930; Csalogovits et al. 1931).

Charcoals from the upper layer hut base recovered in 1957 were sampled for radiocarbon dating and resulted in $17760 \pm 150 \mathrm{BP}$ (GrN-1959) (VogeL, WaterbolK 1964). The lower layer also yielded charcoal samples from one of the hearth features and resulted in $18900 \pm 100$ (GrN-1783) (Vogel, Waterbolk 1964). Mollusk shells and charcoals taken from near the excavation area also were dated to $18510 \pm 160$ (Deb-8822) and $19770 \pm 150$ (Deb-8821), respectively (Krolopp, SÜMEGI 2002). These dates were associated with the lower layer. 
Excavations in 1957-1959 recovered sand stone slabs and a number of antler tools including a perforated specimen. Many of the antler tools were made of shed specimens and the burr was worked down. These pieces showed traces of flint knapping and therefore they can be interpreted as soft hammers. The osseous tool assemblage contains a fragment of a polished point, as well.

Due to the unique character of the lithic artifacts, Ságvár Lyukas-domb archaeological site is the eponym for the Late Upper Palaeolithic archaeological culture called Ságvárian, dated to between 20 and 17 k years BP (KozŁowsKi 1979; TolnaIDoвosi 2001).

Besides its archaeological importance, the site appeared in the terminology of the Late Pleistocene period in Hungary. While Gábori-CsánK (1978) concluded that the organic material rich humic levels at Ságvár were equal to the Lascaux interstadial in France and henceforth called this period Lascaux-Ságvár interstadial. VöRös (1982) pointed out no correspondence to interstadial features on the basis of the faunal remains. The land snail fauna analysis (KrolOPP, SüMEGI 2002) claimed that the human occupation took place during a cold and humid climate with the mean temperature of July 12-12.6 Celsius. This result supports the conclusion of the faunal analysis mentioned above which also did not reveal real interstadial features, as well as the analysis of the loess stratum (BösKen et al. 2018). The age of the site is most probably the Last Glacial Maximum between 21 and $17 \mathrm{ka}$ BP.

The lithic assemblage of Ságvár has undergone a systematic refitting analysis for breakage surfaces and removal negatives. A total of $40 \%$ of the refitted flakes, blades and cores derived from the two archaeological layers (LeNGyel 2010). The high percentage of interlayer refitting at Ságvár suggested that the lithic material of the two archaeological layers most probably is the product of a single knapping activity and therefore they may constitute a single lithic industry.

The lithic assemblage studied here was excavated in 1957-1959 and due to the results of refitting, the two layers were studied unseparated.

\section{Raw materials}

The largest portion of the lithic raw materials are of regional origin from the northern Transdanubia (Bakony, Gerecse, and Vértes Mountains) (Tab. 100). These are chiefly different types of radiolarite, but there are some limnic silicite and silicified lime stone types. The southern Transdanubia was also exploited for radiolarites from Mecsek Mountains. Regional materials weigh $18.047 \mathrm{~kg}$ and yielded 125.1 item per kilogram.

The distant lithic raw materials are mostly limnic silicites. Distant sources are located in Cserhát and Mátra Mountains. These weigh $8.6 \mathrm{~kg}$ and yielded 86.1 item per kilogram.

A small portion of the assemblage, $42 \mathrm{~g}$, was made of flints of Jurassic and Cretaceous transcarpathian sources, which yielded 380.1 item per kilogram. 


\section{Blade tool production}

Basically, all three types of raw materials produced all necessary elements of lithic production. TC material contains the least elements of the full sequence, which is due to that this raw material was used to make flakes and the missing elements are all from blade technology. The other two material groups produced all elements of both flake and blade debitage.

Within each group of raw material flakes are dominant (Tab. 101). Blade cores are also less numerous than flake cores, and within the distant material group the flake cores significantly outnumber blade cores. Blade debitage elements were found in regional and distant material processing, while the TC production seems to have been devoted to making flakes (Tab. 101).

The blades are the minority in the assemblage, and each raw material yielded a small amount of blades. The blade production is chiefly unidirectional (Tab. 102) exploiting single platform cores (Tab. 103). This feature is uniform in the whole assemblage regardless to raw material type. Plain platforms are the most frequent, but preparation that results in dihedral and facetted platforms also occurred (Tab. 104). Blades were generally detached by soft hammer percussion (Tab. 105).

Regional blades tend to be shorter and thinner than distant ones (Tab. 106). The t-test showed this difference significant (Tab. 107).

The blades make up only $26.5 \%$ of the toolkit (Tab. 108), but while $13.8 \%$ of all flakes are tools, $19.0 \%$ of the blades were used to make tools (Tab. 109).

The blade tools are smaller than blank blades (Tab. 110), but the t-test found no difference in the mean length, width and thickness (Tab. 111). However, there is a difference between the tool sizes by raw materials: regional blade tools are thinner than distant blade tools (Tab. 112).

Blades were commonly edge retouched tools and burins, and they are the sole blanks for backed tools (Tab. 113). The armature is composed of a few backed bladelets and a single backed-truncated bladelet, all made of regional raw material (Tab. 114).

\section{Flake tool production}

Flakes are the most abundant products of the industry. The flake debitage used multiple striking platform cores (Tab. 115), but unidirectional scars dominate the flakes (Tab. 116). Flake platforms are primarily plain (Tab. 117), and hard hammer percussion is the dominant technique (Tab. 118).

The mean length, width and thickness of the flakes (Tab. 119) differ by raw materials. ANOVA (Tab. 120) showed that regional flakes are shorter, narrower and thinner than distant flakes, but these two do not differ from TC flakes.

Flakes are the main blanks for tools (Tab. 108), but a smaller portion of the total flake assemblage was used up to make tools than that of the blades (Tab. 109). The mean size of the flake tools is greater than that of the blanks (Tab. 121). The t-test found this difference significant among regional flakes, also the mean thickness of distant flakes tools is greater than that of the blank flakes (Tab. 122). The t-test also 
showed that the sizes of the flakes by raw materials are different when regional and distant materials are compared (Tab. 123). The only TC flake tool was excluded from this analysis.

Flake tools are most often burin, splintered item and endscraper (Tab. 124). There is a significant amount of tools made on debris (Tab. 108), especially of the regional raw material. The tool type list is similar to that of the flake tools, but tools made by debris are chiefly splintered items (Tab. 125). Rejuvenating flakes are few among the tools, these are mostly end-scrapers. Besides the flakes numerous debris and a few blade core platform rejuvenating flakes are also tools. A few flake and blade cores are also tools.

Table 100. Ságvár lithic raw material composition by weight in grams

\begin{tabular}{|l|c|c|c|c|c|}
\hline Raw material & Blade & Flake+debris & Core & Total & $\%$ \\
\hline Regional & 537 & 6033 & 11477 & 18047 & 67.46794 \\
\hline Within regional \% & 2.975564 & 33.42938 & 63.59506 & 100 & \\
\hline Distant & 179 & 4745 & 3736 & 8660 & 32.37504 \\
\hline Within distant \% & 2.066975 & 54.79215 & 43.14088 & 100 & \\
\hline Transcarpathian & 4 & 17 & 21 & 42 & 0.157015 \\
\hline Within transcarpathian \% & 9.52381 & 40.47619 & 50 & 100 & \\
\hline Total & 720 & 10795 & 15234 & 26749 & 100 \\
\hline
\end{tabular}

Table 101. Ságvár lithic assemblage composition by raw material types and technological categories

\begin{tabular}{|c|c|c|c|c|c|c|}
\hline & \multicolumn{3}{|c|}{ Raw material } & \multirow{2}{*}{ Total } \\
\hline & & & regional & distant & transcarpathian & \\
\hline \multirow[t]{16}{*}{ Class } & \multirow[t]{2}{*}{ flake } & count & 857 & 346 & 11 & 1214 \\
\hline & & $\%$ within raw material & $38.0 \%$ & $46.4 \%$ & $68.8 \%$ & $40.2 \%$ \\
\hline & \multirow[t]{2}{*}{ blade } & count & 318 & 72 & 1 & 391 \\
\hline & & $\%$ within raw material & $14.1 \%$ & $9.7 \%$ & $6.3 \%$ & $12.9 \%$ \\
\hline & \multirow[t]{2}{*}{ debris } & count & 862 & 251 & 3 & 1116 \\
\hline & & $\%$ within raw material & $38.2 \%$ & $33.6 \%$ & $18.8 \%$ & $37.0 \%$ \\
\hline & \multirow{2}{*}{$\begin{array}{l}\text { rejuvenating } \\
\text { flake }\end{array}$} & count & 18 & 9 & 0 & 27 \\
\hline & & $\%$ within raw material & $0.8 \%$ & $1.2 \%$ & $0.0 \%$ & $0.9 \%$ \\
\hline & \multirow[t]{2}{*}{ crest } & count & 9 & 1 & 0 & 10 \\
\hline & & $\%$ within raw material & $0.4 \%$ & $0.1 \%$ & $0.0 \%$ & $0.3 \%$ \\
\hline & \multirow[t]{2}{*}{ neo-crest } & count & 23 & 7 & 0 & 30 \\
\hline & & $\%$ within raw material & $1.0 \%$ & $0.9 \%$ & $0.0 \%$ & $1.0 \%$ \\
\hline & \multirow[t]{2}{*}{ blade core } & count & 83 & 23 & 0 & 106 \\
\hline & & $\%$ within raw material & $3.7 \%$ & $3.1 \%$ & $0.0 \%$ & $3.5 \%$ \\
\hline & \multirow[t]{2}{*}{ flake core } & count & 88 & 37 & 1 & 126 \\
\hline & & $\%$ within raw material & $3.9 \%$ & $5.0 \%$ & $6.3 \%$ & $4.2 \%$ \\
\hline \multirow{2}{*}{\multicolumn{2}{|c|}{ Total }} & count & 2258 & 746 & 16 & 3020 \\
\hline & & $\%$ within raw material & $100.0 \%$ & $100.0 \%$ & $100.0 \%$ & $100.0 \%$ \\
\hline
\end{tabular}


Table 102. Ságvár blade assemblage (complete specimens) dorsal scar pattern frequency by raw material

\begin{tabular}{|c|c|c|c|c|c|}
\hline & \multicolumn{2}{|c|}{ Raw material } & \multirow{2}{*}{ Total } \\
\hline & & & regional & distant & \\
\hline \multirow[t]{10}{*}{ Scars } & \multirow{2}{*}{ unidirectional } & count & 79 & 19 & 98 \\
\hline & & $\%$ within raw material & $54.9 \%$ & $57.6 \%$ & $55.4 \%$ \\
\hline & \multirow[t]{2}{*}{ opposite } & count & 19 & 7 & 26 \\
\hline & & $\%$ within raw material & $13.2 \%$ & $21.2 \%$ & $14.7 \%$ \\
\hline & \multirow[t]{2}{*}{ perpendicular } & count & 36 & 7 & 43 \\
\hline & & $\%$ within raw material & $25.0 \%$ & $21.2 \%$ & $24.3 \%$ \\
\hline & \multirow[t]{2}{*}{ multiple } & count & 9 & 0 & 9 \\
\hline & & $\%$ within raw material & $6.3 \%$ & $0.0 \%$ & $5.1 \%$ \\
\hline & \multirow[t]{2}{*}{ no scar } & count & 1 & 0 & 1 \\
\hline & & $\%$ within raw material & $0.7 \%$ & $0.0 \%$ & $0.6 \%$ \\
\hline \multirow{2}{*}{\multicolumn{2}{|c|}{ Total }} & count & 144 & 33 & 177 \\
\hline & & $\%$ within raw material & $100.0 \%$ & $100.0 \%$ & $100.0 \%$ \\
\hline
\end{tabular}

Table 103. Ságvár blade core types by raw material

\begin{tabular}{|c|c|c|c|c|c|}
\hline & \multicolumn{2}{|c|}{ Raw material } & \multirow{2}{*}{ Total } \\
\hline & & & regional & distant & \\
\hline \multirow[t]{6}{*}{ Types } & \multirow[t]{2}{*}{ unidirectional } & count & 60 & 21 & 81 \\
\hline & & \% within raw material & $72.3 \%$ & $91.3 \%$ & $76.4 \%$ \\
\hline & \multirow[t]{2}{*}{ bidirectional } & count & 8 & 1 & 9 \\
\hline & & \% within raw material & $9.6 \%$ & $4.3 \%$ & $8.5 \%$ \\
\hline & \multirow[t]{2}{*}{ alternate } & count & 15 & 1 & 16 \\
\hline & & $\%$ within raw material & $18.1 \%$ & $4.3 \%$ & $15.1 \%$ \\
\hline \multirow{2}{*}{\multicolumn{2}{|c|}{ Total }} & count & 83 & 23 & 106 \\
\hline & & $\%$ within raw material & $100.0 \%$ & $100.0 \%$ & $100.0 \%$ \\
\hline
\end{tabular}

Table 104. Ságvár blade assemblage (complete specimens) platform type frequency by raw material

\begin{tabular}{|c|c|c|c|c|c|}
\hline & \multicolumn{2}{|c|}{ Raw material } & \multirow{2}{*}{ Total } \\
\hline & & & regional & distant & \\
\hline \multirow[t]{14}{*}{ Platform } & \multirow[t]{2}{*}{ plain } & count & 82 & 19 & 101 \\
\hline & & $\%$ within raw material & $56.9 \%$ & $57.6 \%$ & $57.1 \%$ \\
\hline & \multirow[t]{2}{*}{ dihedral } & count & 9 & 1 & 10 \\
\hline & & $\%$ within raw material & $6.3 \%$ & $3.0 \%$ & $5.6 \%$ \\
\hline & \multirow[t]{2}{*}{ faceted } & count & 14 & 5 & 19 \\
\hline & & $\%$ within raw material & $9.7 \%$ & $15.2 \%$ & $10.7 \%$ \\
\hline & \multirow[t]{2}{*}{ cortical } & count & 6 & 3 & 9 \\
\hline & & $\%$ within raw material & $4.2 \%$ & $9.1 \%$ & $5.1 \%$ \\
\hline & \multirow[t]{2}{*}{ linear } & count & 17 & 3 & 20 \\
\hline & & $\%$ within raw material & $11.8 \%$ & $9.1 \%$ & $11.3 \%$ \\
\hline & \multirow[t]{2}{*}{ punctiform } & count & 12 & 2 & 14 \\
\hline & & $\%$ within raw material & $8.3 \%$ & $6.1 \%$ & $7.9 \%$ \\
\hline & \multirow[t]{2}{*}{ irregular } & count & 4 & 0 & 4 \\
\hline & & $\%$ within raw material & $2.8 \%$ & $0.0 \%$ & $2.3 \%$ \\
\hline \multirow{2}{*}{\multicolumn{2}{|c|}{ Total }} & count & 144 & 33 & 177 \\
\hline & & $\%$ within raw material & $100.0 \%$ & $100.0 \%$ & $100.0 \%$ \\
\hline
\end{tabular}


Table 105. Ságvár blade assemblage (complete specimens) impact point-overhang co-presence by raw material

\begin{tabular}{|c|c|c|c|c|c|}
\hline & \multicolumn{2}{|c|}{ Raw material } & \multirow{2}{*}{ Total } \\
\hline & & & regional & distant & \\
\hline \multirow{8}{*}{$\begin{array}{l}\text { Impact } \\
\text { point-overhang }\end{array}$} & \multirow{2}{*}{ none } & count & 88 & 17 & 105 \\
\hline & & $\%$ within raw material & $61.1 \%$ & $51.5 \%$ & $59.3 \%$ \\
\hline & \multirow[t]{2}{*}{ yes-no } & count & 15 & 4 & 19 \\
\hline & & $\%$ within raw material & $10.4 \%$ & $12.1 \%$ & $10.7 \%$ \\
\hline & \multirow[t]{2}{*}{ yes-yes } & count & 8 & 1 & 9 \\
\hline & & $\%$ within raw material & $5.6 \%$ & $3.0 \%$ & $5.1 \%$ \\
\hline & \multirow[t]{2}{*}{ no-yes } & count & 33 & 11 & 44 \\
\hline & & $\%$ within raw material & $22.9 \%$ & $33.3 \%$ & $24.9 \%$ \\
\hline \multirow{2}{*}{\multicolumn{2}{|c|}{ Total }} & count & 144 & 33 & 177 \\
\hline & & $\%$ within raw material & $100.0 \%$ & $100.0 \%$ & $100.0 \%$ \\
\hline
\end{tabular}

Table 106. Ságvár blade assemblage (complete specimens) length, width and thickness by raw material

\begin{tabular}{|l|l|c|c|c|}
\hline Raw material & & Length [mm] & Width [mm] & Thickness [mm] \\
\hline \multirow{5}{*}{ Regional } & Minimum & 3.80 & 4.00 & 1.20 \\
\cline { 2 - 5 } & Maximum & 73.50 & 32.40 & 27.70 \\
\cline { 2 - 5 } & Mean & 30.8167 & 12.7910 & 5.3944 \\
\cline { 2 - 5 } & Median & 29.2000 & 12.3000 & 4.6000 \\
\cline { 2 - 5 } & $\mathrm{N}$ & 144 & 144 & 144 \\
\cline { 2 - 5 } & Std. deviation & 11.36026 & 4.82232 & 3.30490 \\
\hline \multirow{5}{*}{ Distant } & Minimum & 20.30 & 6.10 & 2.00 \\
\cline { 2 - 5 } & Maximum & 67.40 & 22.80 & 17.50 \\
\cline { 2 - 5 } & Mean & 36.9636 & 14.4212 & 6.1879 \\
\cline { 2 - 5 } & Median & 34.9000 & 13.7000 & 33 \\
\cline { 2 - 5 } & $\mathrm{N}$ & 33 & 33 & 3.33923 \\
\cline { 2 - 5 } & Std. deviation & 12.30886 & 4.70809 & 27.20 \\
\hline & Minimum & 3.80 & 4.00 & 5.7288 \\
\cline { 2 - 5 } & Maximum & 73.50 & 32.40 & 5.0000 \\
\cline { 2 - 5 } & Mean & 31.9627 & 13.0949 & 177 \\
\cline { 2 - 5 } & Median & 10.0000 & 12.7000 & 3.37526 \\
\cline { 2 - 5 } & $\mathrm{N}$ & 17.75450 & 4.83013 & \\
\cline { 2 - 5 } & Std. deviation & \multicolumn{3}{|c|}{} \\
\hline
\end{tabular}

Table 107. Ságvár blade assemblage (complete specimens) t-test to compare length, width and thickness by raw materials

\begin{tabular}{|c|c|c|c|c|c|c|c|}
\hline \multicolumn{8}{|c|}{ Independent Samples Test } \\
\hline & \multicolumn{2}{|c|}{$\begin{array}{l}\text { Levene's Test } \\
\text { for Equality } \\
\text { of Variances }\end{array}$} & \multicolumn{5}{|c|}{ t-test for Equality of Means } \\
\hline & $\mathrm{F}$ & Sig. & $\mathrm{t}$ & df & $\begin{array}{c}\text { Sig. } \\
\text { (2-tailed) }\end{array}$ & $\begin{array}{c}\text { Mean } \\
\text { difference }\end{array}$ & $\begin{array}{l}\text { Std. error } \\
\text { difference }\end{array}$ \\
\hline \multirow{2}{*}{$\begin{array}{l}\text { Length } \\
{[\mathrm{mm}]}\end{array}$} & 0.552 & 0.458 & -2.760 & 175 & 0.006 & -6.14697 & 2.22709 \\
\hline & & & -2.624 & 45.326 & 0.012 & -6.14697 & 2.34251 \\
\hline \multirow{2}{*}{$\begin{array}{l}\text { Width } \\
{[\mathrm{mm}]}\end{array}$} & 0.234 & 0.629 & -1.759 & 175 & 0.080 & -1.63024 & 0.92670 \\
\hline & & & -1.786 & 48.608 & 0.080 & -1.63024 & 0.91279 \\
\hline \multirow{2}{*}{$\begin{array}{l}\text { Thickness } \\
{[\mathrm{mm}]}\end{array}$} & 1.563 & 0.213 & -2.806 & 175 & 0.006 & -1.79343 & 0.63905 \\
\hline & & & -2.788 & 47.444 & 0.008 & -1.79343 & 0.64323 \\
\hline
\end{tabular}


Lithic analysis of the Middle and Late Upper Palaeolithic in Hungary

Table 108. Ságvár tool assemblage product composition by raw materials

\begin{tabular}{|c|c|c|c|c|c|}
\hline & \multicolumn{3}{|c|}{ Raw material } & \multirow{2}{*}{ Total } \\
\hline & & regional & distant & transcarpathian & \\
\hline \multirow[t]{2}{*}{ Flake } & count & 122 & 44 & 1 & 167 \\
\hline & $\%$ within raw material & $50.8 \%$ & $64.7 \%$ & $100.0 \%$ & $54.0 \%$ \\
\hline \multirow[t]{2}{*}{ Blade } & count & 72 & 10 & 0 & 82 \\
\hline & $\%$ within raw material & $30.0 \%$ & $14.7 \%$ & $0.0 \%$ & $26.5 \%$ \\
\hline \multirow[t]{2}{*}{ Debris } & count & 42 & 13 & 0 & 55 \\
\hline & \% within raw material & $17.5 \%$ & $19.1 \%$ & $0.0 \%$ & $17.8 \%$ \\
\hline \multirow{2}{*}{$\begin{array}{l}\text { Rejuvenating } \\
\text { flake }\end{array}$} & count & 4 & 1 & 0 & 5 \\
\hline & \% within raw material & $1.7 \%$ & $1.5 \%$ & $0.0 \%$ & $1.6 \%$ \\
\hline \multirow{2}{*}{ Total } & count & 240 & 68 & 1 & 309 \\
\hline & $\%$ within raw material & $100.0 \%$ & $100.0 \%$ & $100.0 \%$ & $100.0 \%$ \\
\hline
\end{tabular}

Table 109. Ságvár knapped product frequency in tool assemblage by raw materials

\begin{tabular}{|c|c|c|c|c|c|c|c|}
\hline & \multicolumn{3}{|c|}{ Raw material } & \multirow{2}{*}{ Total } \\
\hline & & & & regional & distant & transcarpathian & \\
\hline \multirow[t]{6}{*}{ Flake } & \multirow[t]{4}{*}{ state } & \multirow[t]{2}{*}{ blank } & count & 735 & 302 & 10 & 1047 \\
\hline & & & $\%$ within raw material & $85.8 \%$ & $87.3 \%$ & $90.9 \%$ & $86.2 \%$ \\
\hline & & \multirow[t]{2}{*}{ tool } & count & 122 & 44 & 1 & 167 \\
\hline & & & $\%$ within raw material & $14.2 \%$ & $12.7 \%$ & $9.1 \%$ & $13.8 \%$ \\
\hline & \multirow{2}{*}{\multicolumn{2}{|c|}{ total }} & count & 857 & 346 & 11 & 1214 \\
\hline & & & $\%$ within raw material & $100.0 \%$ & $100.0 \%$ & $100.0 \%$ & $100.0 \%$ \\
\hline \multirow[t]{6}{*}{ Blade } & \multirow[t]{4}{*}{ state } & \multirow[t]{2}{*}{ blank } & count & 278 & 70 & 1 & 349 \\
\hline & & & $\%$ within raw material & $79.4 \%$ & $87.5 \%$ & $100.0 \%$ & $81.0 \%$ \\
\hline & & \multirow[t]{2}{*}{ tool } & count & 72 & 10 & 0 & 82 \\
\hline & & & $\%$ within raw material & $20.6 \%$ & $12.5 \%$ & $0.0 \%$ & $19.0 \%$ \\
\hline & \multirow{2}{*}{\multicolumn{2}{|c|}{ total }} & count & 350 & 80 & 1 & 431 \\
\hline & & & $\%$ within raw material & $100.0 \%$ & $100.0 \%$ & $100.0 \%$ & $100.0 \%$ \\
\hline \multirow[t]{6}{*}{ Debris } & \multirow[t]{4}{*}{ state } & \multirow[t]{2}{*}{ blank } & count & 820 & 238 & 3 & 1061 \\
\hline & & & $\%$ within raw material & $95.1 \%$ & $94.8 \%$ & $100.0 \%$ & $95.1 \%$ \\
\hline & & \multirow[t]{2}{*}{ tool } & count & 42 & 13 & 0 & 55 \\
\hline & & & $\%$ within raw material & $4.9 \%$ & $5.2 \%$ & $0.0 \%$ & $4.9 \%$ \\
\hline & \multirow{2}{*}{\multicolumn{2}{|c|}{ total }} & count & 862 & 251 & 3 & 1116 \\
\hline & & & $\%$ within raw material & $100.0 \%$ & $100.0 \%$ & $100.0 \%$ & $100.0 \%$ \\
\hline \multirow{6}{*}{$\begin{array}{l}\text { Rejuvenating } \\
\text { flake }\end{array}$} & \multirow[t]{4}{*}{ state } & \multirow[t]{2}{*}{ blank } & count & 14 & 8 & & 22 \\
\hline & & & \% within raw material & $77.8 \%$ & $88.9 \%$ & & $81.5 \%$ \\
\hline & & \multirow[t]{2}{*}{ tool } & count & 4 & 1 & & 5 \\
\hline & & & $\%$ within raw material & $22.2 \%$ & $11.1 \%$ & & $18.5 \%$ \\
\hline & \multirow{2}{*}{\multicolumn{2}{|c|}{ total }} & count & 18 & 9 & & 27 \\
\hline & & & $\%$ within raw material & $100.0 \%$ & $100.0 \%$ & & $100.0 \%$ \\
\hline
\end{tabular}


Table 110. Ságvár blade tool assemblage length, width and thickness by raw materials

\begin{tabular}{|c|c|c|c|c|}
\hline \multicolumn{2}{|c|}{ Raw material } & Length [mm] & Width [mm] & Thickness [mm] \\
\hline \multirow[t]{6}{*}{ Regional } & Minimum & 10.30 & 4.00 & 1.80 \\
\hline & Maximum & 78.50 & 28.80 & 15.60 \\
\hline & Mean & 28.0085 & 12.9028 & 5.5000 \\
\hline & Median & 25.4000 & 12.4000 & 5.0000 \\
\hline & $\mathrm{N}$ & 71 & 71 & 71 \\
\hline & Std. deviation & 11.41980 & 5.29954 & 2.72229 \\
\hline \multirow[t]{6}{*}{ Distant } & Minimum & 19.30 & 6.50 & 3.50 \\
\hline & Maximum & 59.40 & 33.60 & 17.80 \\
\hline & Mean & 33.4800 & 18.0200 & 8.6600 \\
\hline & Median & 31.4500 & 18.3000 & 8.1500 \\
\hline & $\mathrm{N}$ & 10 & 10 & 10 \\
\hline & Std. deviation & 13.09926 & 9.49313 & 3.92349 \\
\hline \multirow[t]{6}{*}{ Total } & Minimum & 10.30 & 4.00 & 1.80 \\
\hline & Maximum & 78.50 & 33.60 & 17.80 \\
\hline & Mean & 28.6840 & 13.5346 & 5.8901 \\
\hline & Median & 26.0000 & 12.5000 & 5.4000 \\
\hline & $\mathrm{N}$ & 81 & 81 & 81 \\
\hline & Std. deviation & 11.69165 & 6.13042 & 3.05130 \\
\hline
\end{tabular}

Table 111. Ságvár blade blank and tool length, width and thickness mean t-test comparison by raw material

\begin{tabular}{|c|c|c|c|c|c|c|c|c|}
\hline \multicolumn{9}{|c|}{ Independent Samples Test } \\
\hline \multirow{2}{*}{\multicolumn{2}{|c|}{ Raw material }} & \multicolumn{2}{|c|}{$\begin{array}{l}\text { Levene's Test } \\
\text { for Equality } \\
\text { of Variances }\end{array}$} & \multicolumn{5}{|c|}{ t-test for Equality of Means } \\
\hline & & $\mathrm{F}$ & Sig. & $\mathrm{t}$ & df & $\begin{array}{c}\text { Sig. } \\
\text { (2-tailed) }\end{array}$ & $\begin{array}{c}\text { Mean } \\
\text { difference }\end{array}$ & $\begin{array}{l}\text { Std. error } \\
\text { difference }\end{array}$ \\
\hline \multirow[t]{6}{*}{ Regional } & \multirow{2}{*}{$\begin{array}{l}\text { length } \\
{[\mathrm{mm}]}\end{array}$} & 0.319 & 0.573 & 1.702 & 213 & 0.090 & 2.80822 & 1.65024 \\
\hline & & & & 1.699 & 138.799 & 0.092 & 2.80822 & 1.65318 \\
\hline & \multirow{2}{*}{$\begin{array}{l}\text { width } \\
{[\mathrm{mm}]}\end{array}$} & 1.906 & 0.169 & -0.155 & 213 & 0.877 & -0.11184 & 0.72278 \\
\hline & & & & -0.150 & 128.351 & 0.881 & -0.11184 & 0.74636 \\
\hline & \multirow{2}{*}{$\begin{array}{l}\text { thickness } \\
{[\mathrm{mm}]}\end{array}$} & 0.003 & 0.956 & -0.233 & 213 & 0.816 & -0.10556 & 0.45323 \\
\hline & & & & -0.249 & 165.833 & 0.804 & -0.10556 & 0.42453 \\
\hline \multirow[t]{6}{*}{ Distant } & \multirow{2}{*}{$\begin{array}{l}\text { length } \\
{[\mathrm{mm}]}\end{array}$} & 0.002 & 0.961 & 0.773 & 41 & 0.444 & 3.48364 & 4.50737 \\
\hline & & & & 0.747 & 14.175 & 0.467 & 3.48364 & 4.66371 \\
\hline & \multirow{2}{*}{$\begin{array}{l}\text { width } \\
{[\mathrm{mm}]}\end{array}$} & 10.088 & 0.003 & -1.637 & 41 & 0.109 & -3.59879 & 2.19818 \\
\hline & & & & -1.156 & 10.375 & 0.273 & -3.59879 & 3.11186 \\
\hline & \multirow{2}{*}{$\begin{array}{l}\text { thickness } \\
{[\mathrm{mm}]}\end{array}$} & 0.009 & 0.925 & -1.173 & 41 & 0.247 & -1.47212 & 1.25472 \\
\hline & & & & -1.074 & 13.206 & 0.302 & -1.47212 & 1.37014 \\
\hline
\end{tabular}


Lithic analysis of the Middle and Late Upper Palaeolithic in Hungary

Table 112. Ságvár blade tool length, width and thickness mean comparison by raw materials with t-test

\begin{tabular}{|l|c|c|c|c|c|c|c|}
\hline \multicolumn{1}{|c|}{} & $\begin{array}{c}\text { Levene's Test } \\
\text { for Equality } \\
\text { of Variances }\end{array}$ & \multicolumn{6}{|c|}{ Independent Samples Test } \\
\cline { 2 - 8 } & $\mathrm{F}$ & Sig. & $\mathrm{t}$ & $\mathrm{df}$ & $\begin{array}{c}\text { Sig. } \\
\text { (2-tailed) }\end{array}$ & $\begin{array}{c}\text { Mean } \\
\text { difference }\end{array}$ & $\begin{array}{c}\text { Std. error } \\
\text { difference }\end{array}$ \\
\cline { 2 - 8 } & 0.437 & 0.510 & -1.394 & 79 & 0.167 & -5.47155 & 3.92596 \\
\hline $\begin{array}{l}\text { Length } \\
{[\mathrm{mm}]}\end{array}$ & & & -1.255 & 11.014 & 0.235 & -5.47155 & 4.35842 \\
\hline $\begin{array}{l}\text { Width } \\
{[\mathrm{mm}]}\end{array}$ & 8.709 & 0.004 & -2.555 & 79 & 0.013 & -5.11718 & 2.00258 \\
\hline $\begin{array}{l}\text { Thickness } \\
{[\mathrm{mm}]}\end{array}$ & 1.150 & 0.287 & -3.243 & 79 & 0.002 & -3.16000 & 0.97428 \\
\cline { 2 - 8 } & & & -2.465 & 10.256 & 0.033 & -3.16000 & 1.28209 \\
\hline
\end{tabular}

Table 113. Ságvár blade tool types by raw material

\begin{tabular}{|c|c|c|c|c|c|}
\hline & \multicolumn{2}{|c|}{ Raw material } & \multirow{2}{*}{ Total } \\
\hline & & & regional & distant & \\
\hline \multirow[t]{18}{*}{ Tooltypes } & \multirow[t]{2}{*}{ endscraper } & count & 10 & 0 & 10 \\
\hline & & $\%$ within raw material & $13.9 \%$ & $0.0 \%$ & $12.2 \%$ \\
\hline & \multirow[t]{2}{*}{ burin } & count & 14 & 2 & 16 \\
\hline & & \% within raw material & $19.4 \%$ & $20.0 \%$ & $19.5 \%$ \\
\hline & \multirow[t]{2}{*}{ retouched } & count & 27 & 3 & 30 \\
\hline & & $\%$ within raw material & $37.5 \%$ & $30.0 \%$ & $36.6 \%$ \\
\hline & \multirow{2}{*}{ borer } & count & 1 & 0 & 1 \\
\hline & & \% within raw material & $1.4 \%$ & $0.0 \%$ & $1.2 \%$ \\
\hline & \multirow{2}{*}{$\begin{array}{l}\text { splintered } \\
\text { piece }\end{array}$} & count & 5 & 0 & 5 \\
\hline & & $\%$ within raw material & $6.9 \%$ & $0.0 \%$ & $6.1 \%$ \\
\hline & \multirow[t]{2}{*}{ truncation } & count & 1 & 0 & 1 \\
\hline & & $\%$ within raw material & $1.4 \%$ & $0.0 \%$ & $1.2 \%$ \\
\hline & \multirow{2}{*}{$\begin{array}{l}\text { notched- } \\
\text { denticulated }\end{array}$} & count & 4 & 5 & 9 \\
\hline & & $\%$ within raw material & $5.6 \%$ & $50.0 \%$ & $11.0 \%$ \\
\hline & \multirow[t]{2}{*}{ composite } & count & 2 & 0 & 2 \\
\hline & & $\%$ within raw material & $2.8 \%$ & $0.0 \%$ & $2.4 \%$ \\
\hline & \multirow[t]{2}{*}{ armature } & count & 8 & 0 & 8 \\
\hline & & $\%$ within raw material & $11.1 \%$ & $0.0 \%$ & $9.8 \%$ \\
\hline \multirow{2}{*}{\multicolumn{2}{|c|}{ Total }} & count & 72 & 10 & 82 \\
\hline & & $\%$ within raw material & $100.0 \%$ & $100.0 \%$ & $100.0 \%$ \\
\hline
\end{tabular}

Table 114. Ságvár armature types by raw material

\begin{tabular}{|c|c|c|c|c|}
\hline & Raw material & \multirow{2}{*}{ Total } \\
\hline & & & regional & \\
\hline \multirow[t]{4}{*}{ Armature } & \multirow[t]{2}{*}{ backed } & count & 7 & 7 \\
\hline & & $\%$ within raw material & $87.5 \%$ & $87.5 \%$ \\
\hline & \multirow{2}{*}{$\begin{array}{l}\text { backed- } \\
\text { truncated }\end{array}$} & count & 1 & 1 \\
\hline & & $\%$ within raw material & $12.5 \%$ & $12.5 \%$ \\
\hline \multirow{2}{*}{\multicolumn{2}{|c|}{ Total }} & count & 8 & 8 \\
\hline & & \% within raw material & $100.0 \%$ & $100.0 \%$ \\
\hline
\end{tabular}


Table 115. Ságvár flake core types by raw material

\begin{tabular}{|c|c|c|c|c|c|c|}
\hline & \multicolumn{3}{|c|}{ Raw material } & \multirow{2}{*}{ Total } \\
\hline & & & regional & distant & transcarpathian & \\
\hline \multirow[t]{6}{*}{ Types } & \multirow[t]{2}{*}{ unidirectional } & count & 24 & 10 & 0 & 34 \\
\hline & & $\%$ within raw material & $26.4 \%$ & $29.4 \%$ & $0.0 \%$ & $27.0 \%$ \\
\hline & \multirow[t]{2}{*}{ bidirectional } & count & 16 & 1 & 1 & 18 \\
\hline & & $\%$ within raw material & $17.6 \%$ & $2.9 \%$ & $100.0 \%$ & $14.3 \%$ \\
\hline & \multirow[t]{2}{*}{ multidirectional } & count & 51 & 23 & 0 & 74 \\
\hline & & $\%$ within raw material & $56.0 \%$ & $67.6 \%$ & $0.0 \%$ & $58.7 \%$ \\
\hline \multirow{2}{*}{\multicolumn{2}{|c|}{ Total }} & count & 91 & 34 & 1 & 126 \\
\hline & & $\%$ within raw material & $100.0 \%$ & $100.0 \%$ & $100.0 \%$ & $100.0 \%$ \\
\hline
\end{tabular}

Table 116. Ságvár flake assemblage (complete specimens) dorsal scar pattern frequency by raw material

\begin{tabular}{|c|c|c|c|c|c|c|}
\hline & \multicolumn{3}{|c|}{ Raw material } & \multirow{2}{*}{ Total } \\
\hline & & & regional & distant & transcarpathian & \\
\hline \multirow{10}{*}{ Scars } & \multirow[t]{2}{*}{ unidirectional } & count & 246 & 94 & 3 & 343 \\
\hline & & $\%$ within raw material & $48.4 \%$ & $47.0 \%$ & $42.9 \%$ & $48.0 \%$ \\
\hline & \multirow{2}{*}{ opposite } & count & 70 & 19 & 1 & 90 \\
\hline & & $\%$ within raw material & $13.8 \%$ & $9.5 \%$ & $14.3 \%$ & $12.6 \%$ \\
\hline & \multirow[t]{2}{*}{ perpendicular } & count & 132 & 52 & 2 & 186 \\
\hline & & $\%$ within raw material & $26.0 \%$ & $26.0 \%$ & $28.6 \%$ & $26.0 \%$ \\
\hline & \multirow[t]{2}{*}{ multiple } & count & 59 & 32 & 1 & 92 \\
\hline & & $\%$ within raw material & $11.6 \%$ & $16.0 \%$ & $14.3 \%$ & $12.9 \%$ \\
\hline & \multirow[t]{2}{*}{ no scar } & count & 1 & 3 & 0 & 4 \\
\hline & & $\%$ within raw material & $0.2 \%$ & $1.5 \%$ & $0.0 \%$ & $0.6 \%$ \\
\hline \multirow{2}{*}{\multicolumn{2}{|c|}{ Total }} & count & 508 & 200 & 7 & 715 \\
\hline & & $\%$ within raw material & $100.0 \%$ & $100.0 \%$ & $100.0 \%$ & $100.0 \%$ \\
\hline
\end{tabular}

Table 117. Ságvár flake assemblage (complete specimens) platform type frequency by raw material

\begin{tabular}{|c|c|c|c|c|c|c|}
\hline & \multicolumn{3}{|c|}{ Raw material } & \multirow{2}{*}{ Total } \\
\hline & & & regional & distant & transcarpathian & \\
\hline \multirow[t]{16}{*}{ Platform } & \multirow[t]{2}{*}{ plain } & count & 289 & 128 & 4 & 421 \\
\hline & & $\%$ within raw material & $56.9 \%$ & $64.0 \%$ & $57.1 \%$ & $58.9 \%$ \\
\hline & \multirow[t]{2}{*}{ dihedral } & count & 39 & 16 & 1 & 56 \\
\hline & & \% within raw material & $7.7 \%$ & $8.0 \%$ & $14.3 \%$ & $7.8 \%$ \\
\hline & \multirow[t]{2}{*}{ faceted } & count & 53 & 9 & 0 & 62 \\
\hline & & $\%$ within raw material & $10.4 \%$ & $4.5 \%$ & $0.0 \%$ & $8.7 \%$ \\
\hline & \multirow[t]{2}{*}{ cortical } & count & 29 & 19 & 0 & 48 \\
\hline & & \% within raw material & $5.7 \%$ & $9.5 \%$ & $0.0 \%$ & $6.7 \%$ \\
\hline & \multirow[t]{2}{*}{ linear } & count & 62 & 13 & 1 & 76 \\
\hline & & $\%$ within raw material & $12.2 \%$ & $6.5 \%$ & $14.3 \%$ & $10.6 \%$ \\
\hline & \multirow{2}{*}{$\begin{array}{l}\text { debitage } \\
\text { surface }\end{array}$} & count & 0 & 1 & 0 & 1 \\
\hline & & $\%$ within raw material & $0.0 \%$ & $0.5 \%$ & $0.0 \%$ & $0.1 \%$ \\
\hline & \multirow[t]{2}{*}{ punctiform } & count & 12 & 1 & 0 & 13 \\
\hline & & \% within raw material & $2.4 \%$ & $0.5 \%$ & $0.0 \%$ & $1.8 \%$ \\
\hline & \multirow[t]{2}{*}{ irregular } & count & 24 & 13 & 1 & 38 \\
\hline & & $\%$ within raw material & $4.7 \%$ & $6.5 \%$ & $14.3 \%$ & $5.3 \%$ \\
\hline \multirow{2}{*}{\multicolumn{2}{|c|}{ Total }} & count & 508 & 200 & 7 & 715 \\
\hline & & $\%$ within raw material & $100.0 \%$ & $100.0 \%$ & $100.0 \%$ & $100.0 \%$ \\
\hline
\end{tabular}


Table 118. Ságvár flake assemblage (complete specimens) impact point-overhang co-presence by raw material

\begin{tabular}{|c|c|c|c|c|c|c|}
\hline & \multicolumn{3}{|c|}{ Raw material } & \multirow{2}{*}{ Total } \\
\hline & & & regional & distant & transcarpathian & \\
\hline \multirow{8}{*}{$\begin{array}{l}\text { Impact } \\
\text { point-overhang }\end{array}$} & \multirow[t]{2}{*}{ none } & count & 187 & 60 & 3 & 250 \\
\hline & & $\%$ within raw material & $36.8 \%$ & $30.0 \%$ & $42.9 \%$ & $35.0 \%$ \\
\hline & \multirow[t]{2}{*}{ yes-no } & count & 65 & 25 & 2 & 92 \\
\hline & & $\%$ within raw material & $12.8 \%$ & $12.5 \%$ & $28.6 \%$ & $12.9 \%$ \\
\hline & \multirow[t]{2}{*}{ yes-yes } & count & 86 & 39 & 0 & 125 \\
\hline & & $\%$ within raw material & $16.9 \%$ & $19.5 \%$ & $0.0 \%$ & $17.5 \%$ \\
\hline & \multirow[t]{2}{*}{ no-yes } & count & 170 & 76 & 2 & 248 \\
\hline & & $\%$ within raw material & $33.5 \%$ & $38.0 \%$ & $28.6 \%$ & $34.7 \%$ \\
\hline \multirow{2}{*}{\multicolumn{2}{|c|}{ Total }} & count & 508 & 200 & 7 & 715 \\
\hline & & $\%$ within raw material & $100.0 \%$ & $100.0 \%$ & $100.0 \%$ & $100.0 \%$ \\
\hline
\end{tabular}

Table 119. Ságvár flake blank length, width and thickness by raw material

\begin{tabular}{|c|c|c|c|c|}
\hline \multicolumn{2}{|l|}{ Raw material } & Length $[\mathrm{mm}]$ & Width [mm] & Thickness [mm] \\
\hline \multirow[t]{6}{*}{ Regional } & Minimum & 3.20 & 5.50 & .90 \\
\hline & Maximum & 59.50 & 52.60 & 31.60 \\
\hline & Mean & 23.3673 & 18.7679 & 6.2185 \\
\hline & Median & 21.6000 & 17.3000 & 5.2500 \\
\hline & $\mathrm{N}$ & 508 & 508 & 508 \\
\hline & Std. deviation & 9.14829 & 7.70581 & 4.02021 \\
\hline \multirow[t]{6}{*}{ Distant } & Minimum & 11.00 & 10.60 & 1.90 \\
\hline & Maximum & 73.50 & 63.40 & 30.40 \\
\hline & Mean & 33.1260 & 27.8495 & 9.3275 \\
\hline & Median & 32.2500 & 25.5000 & 8.5000 \\
\hline & $\mathrm{N}$ & 200 & 200 & 200 \\
\hline & Std. deviation & 11.87486 & 10.37833 & 4.77363 \\
\hline \multirow[t]{6}{*}{ Transcarpathian } & Minimum & 13.80 & 13.00 & 3.40 \\
\hline & Maximum & 41.60 & 27.70 & 8.50 \\
\hline & Mean & 25.4286 & 23.5714 & 6.0571 \\
\hline & Median & 24.1000 & 25.0000 & 6.4000 \\
\hline & $\mathrm{N}$ & 7 & 7 & 7 \\
\hline & Std. deviation & 10.06921 & 4.90500 & 1.83835 \\
\hline \multirow[t]{6}{*}{ Total } & Minimum & 3.20 & 5.50 & .90 \\
\hline & Maximum & 73.50 & 63.40 & 31.60 \\
\hline & Mean & 26.1172 & 21.3552 & 7.0866 \\
\hline & Median & 23.7000 & 19.5000 & 5.8000 \\
\hline & $\mathrm{N}$ & 715 & 715 & 715 \\
\hline & Std. deviation & 10.89616 & 9.43459 & 4.45075 \\
\hline
\end{tabular}


Table 120. Ságvár flake blank length, width and thickness mean comparison by raw materials with ANOVA and the Tukey post hoc

ANOVA

\begin{tabular}{|l|l|r|r|r|r|c|}
\hline \multicolumn{2}{|c|}{} & \multicolumn{1}{|c|}{$\begin{array}{c}\text { Sum of } \\
\text { squares }\end{array}$} & df & $\begin{array}{c}\text { Mean } \\
\text { square }\end{array}$ & F & Sig. \\
\hline \multirow{2}{*}{$\begin{array}{l}\text { Length } \\
{[\mathrm{mm}]}\end{array}$} & between groups & 13669.382 & 2 & 6834.691 & 68.442 & 0.000 \\
\cline { 2 - 7 } & within groups & 71101.237 & 712 & 99.861 & & \\
\cline { 2 - 7 } & Total & 84770.618 & 714 & & & \\
\hline \multirow{2}{*}{$\begin{array}{l}\text { Width } \\
{[\mathrm{mm}]}\end{array}$} & between groups & 11870.147 & 2 & 5935.073 & 81.762 & 0.000 \\
\cline { 2 - 7 } & within groups & 51684.021 & 712 & 72.590 & & \\
\cline { 2 - 7 } & Total & 63554.168 & 714 & & & \\
\hline \multirow{2}{*}{$\begin{array}{l}\text { Thickness } \\
{[\mathrm{mm}]}\end{array}$} & between groups & 1394.569 & 2 & 697.285 & 38.941 & 0.000 \\
\cline { 2 - 8 } & within groups & 12749.182 & 712 & 17.906 & & \\
\cline { 2 - 7 } & Total & 14143.751 & 714 & & & \\
\hline
\end{tabular}

Multiple Comparisons

Tukey HSD

\begin{tabular}{|c|c|c|c|c|c|c|c|}
\hline \multirow{2}{*}{$\begin{array}{l}\text { Dependent } \\
\text { variable }\end{array}$} & \multirow{2}{*}{ (I) raw material } & \multirow{2}{*}{$\begin{array}{l}(\mathrm{J}) \text { raw } \\
\text { material }\end{array}$} & \multirow{2}{*}{$\begin{array}{c}\text { Mean } \\
\text { difference } \\
(\mathrm{I}-\mathrm{J})\end{array}$} & \multirow{2}{*}{$\begin{array}{l}\text { Std. } \\
\text { error }\end{array}$} & \multirow{2}{*}{ Sig. } & \multicolumn{2}{|c|}{$\begin{array}{l}\text { 95\% Confidence } \\
\text { interval }\end{array}$} \\
\hline & & & & & & $\begin{array}{l}\text { Lower } \\
\text { bound }\end{array}$ & $\begin{array}{l}\text { Upper } \\
\text { bound }\end{array}$ \\
\hline \multirow{6}{*}{$\begin{array}{l}\text { Length } \\
{[\mathrm{mm}]}\end{array}$} & \multirow[t]{2}{*}{ regional } & distant & -9.75868 & 0.83420 & 0.000 & -11.7179 & -7.7995 \\
\hline & & transcarpathian & -2.06125 & 3.80296 & 0.851 & -10.9930 & 6.8705 \\
\hline & \multirow[t]{2}{*}{ distant } & regional & 9.75868 & 0.83420 & 0.000 & 7.7995 & 11.7179 \\
\hline & & transcarpathian & 7.69743 & 3.84255 & 0.112 & -1.3273 & 16.7221 \\
\hline & \multirow[t]{2}{*}{ transcarpathian } & regional & 2.06125 & 3.80296 & 0.851 & -6.8705 & 10.9930 \\
\hline & & distant & -7.69743 & 3.84255 & 0.112 & -16.7221 & 1.3273 \\
\hline \multirow{6}{*}{$\begin{array}{l}\text { Width } \\
{[\mathrm{mm}]}\end{array}$} & \multirow[t]{2}{*}{ regional } & distant & -9.08159 & 0.71123 & 0.000 & -10.7520 & -7.4112 \\
\hline & & transcarpathian & -4.80352 & 3.24236 & 0.300 & -12.4186 & 2.8116 \\
\hline & \multirow[t]{2}{*}{ distant } & regional & 9.08159 & 0.71123 & 0.000 & 7.4112 & 10.7520 \\
\hline & & transcarpathian & 4.27807 & 3.27612 & 0.392 & -3.4163 & 11.9724 \\
\hline & \multirow[t]{2}{*}{ transcarpathian } & regional & 4.80352 & 3.24236 & 0.300 & -2.8116 & 12.4186 \\
\hline & & distant & -4.27807 & 3.27612 & 0.392 & -11.9724 & 3.4163 \\
\hline \multirow{6}{*}{$\begin{array}{l}\text { Thickness } \\
\text { [mm] }\end{array}$} & \multirow[t]{2}{*}{ regional } & distant & -3.10900 & 0.35324 & 0.000 & -3.9386 & -2.2794 \\
\hline & & transcarpathian & 0.16136 & 1.61036 & 0.994 & -3.6208 & 3.9435 \\
\hline & \multirow[t]{2}{*}{ distant } & regional & 3.10900 & 0.35324 & 0.000 & 2.2794 & 3.9386 \\
\hline & & transcarpathian & 3.27036 & 1.62713 & 0.111 & -0.5512 & 7.0919 \\
\hline & \multirow[t]{2}{*}{ transcarpathian } & regional & -0.16136 & 1.61036 & 0.994 & -3.9435 & 3.6208 \\
\hline & & distant & -3.27036 & 1.62713 & 0.111 & -7.0919 & 0.5512 \\
\hline
\end{tabular}


Table 121. Ságvár flake tool length, width and thickness

\begin{tabular}{|c|c|c|c|c|}
\hline \multicolumn{2}{|l|}{ Raw material } & Length $[\mathrm{mm}]$ & Width [mm] & Thickness [mm] \\
\hline \multirow[t]{6}{*}{ Regional } & Minimum & 10.60 & 7.90 & 1.60 \\
\hline & Maximum & 48.50 & 53.80 & 20.70 \\
\hline & Mean & 27.6721 & 20.9779 & 8.3148 \\
\hline & Median & 27.0000 & 19.7500 & 7.7500 \\
\hline & $\mathrm{N}$ & 122 & 122 & 122 \\
\hline & Std. deviation & 8.12857 & 7.75472 & 3.75312 \\
\hline \multirow[t]{6}{*}{ Distant } & Minimum & 15.60 & 9.40 & 5.00 \\
\hline & Maximum & 73.70 & 54.00 & 28.50 \\
\hline & Mean & 33.9636 & 25.3977 & 11.5477 \\
\hline & Median & 33.6500 & 24.1000 & 11.1000 \\
\hline & $\mathrm{N}$ & 44 & 44 & 44 \\
\hline & Std. deviation & 9.13682 & 9.38129 & 4.36793 \\
\hline \multirow[t]{6}{*}{ Transcarpathian } & Minimum & 23.50 & 20.80 & 8.50 \\
\hline & Maximum & 23.50 & 20.80 & 8.50 \\
\hline & Mean & 23.5000 & 20.8000 & 8.5000 \\
\hline & Median & 23.5000 & 20.8000 & 8.5000 \\
\hline & $\mathrm{N}$ & 1 & 1 & 1 \\
\hline & Std. deviation & - & - & - \\
\hline \multirow[t]{6}{*}{ Total } & Minimum & 10.60 & 7.90 & 1.60 \\
\hline & Maximum & 73.70 & 54.00 & 28.50 \\
\hline & Mean & 29.3048 & 22.1413 & 9.1677 \\
\hline & Median & 29.0000 & 20.5000 & 8.5000 \\
\hline & $\mathrm{N}$ & 167 & 167 & 167 \\
\hline & Std. deviation & 8.81487 & 8.39331 & 4.15311 \\
\hline
\end{tabular}

Table 122. Ságvár flake blank and tool length, width and thickness mean t-test comparison

\begin{tabular}{|c|c|c|c|c|c|c|c|c|}
\hline \multicolumn{9}{|c|}{ Independent Samples Test } \\
\hline \multirow{2}{*}{\multicolumn{2}{|c|}{ Raw material }} & \multicolumn{2}{|c|}{$\begin{array}{l}\text { Levene's Test } \\
\text { for Equality } \\
\text { of Variances }\end{array}$} & \multicolumn{5}{|c|}{ t-test for Equality of Means } \\
\hline & & $\mathrm{F}$ & Sig. & $\mathrm{t}$ & $\mathrm{df}$ & $\begin{array}{c}\text { Sig. } \\
\text { (2-tailed) }\end{array}$ & $\begin{array}{c}\text { Mean } \\
\text { difference }\end{array}$ & $\begin{array}{l}\text { Std. error } \\
\text { difference }\end{array}$ \\
\hline \multirow[t]{6}{*}{ Regional } & \multirow{2}{*}{$\begin{array}{l}\text { length } \\
{[\mathrm{mm}]}\end{array}$} & 1.002 & 0.317 & -4.765 & 628 & 0.000 & -4.30481 & 0.90346 \\
\hline & & & & -5.122 & 201.364 & 0.000 & -4.30481 & 0.84044 \\
\hline & \multirow{2}{*}{$\begin{array}{l}\text { width } \\
{[\mathrm{mm}]}\end{array}$} & 0.855 & 0.356 & -2.841 & 628 & 0.005 & -2.20996 & 0.77787 \\
\hline & & & & -2.830 & 182.739 & 0.005 & -2.20996 & 0.78090 \\
\hline & \multirow{2}{*}{$\begin{array}{l}\text { thickness } \\
{[\mathrm{mm}]}\end{array}$} & 0.052 & 0.820 & -5.237 & 628 & 0.000 & -2.09625 & 0.40028 \\
\hline & & & & -5.462 & 193.368 & 0.000 & -2.09625 & 0.38376 \\
\hline \multirow[t]{6}{*}{ Distant } & \multirow{2}{*}{$\begin{array}{l}\text { length } \\
{[\mathrm{mm}]}\end{array}$} & 8.473 & 0.004 & -0.440 & 242 & 0.660 & -0.83764 & 1.90432 \\
\hline & & & & -0.519 & 78.553 & 0.605 & -0.83764 & 1.61319 \\
\hline & \multirow{2}{*}{$\begin{array}{l}\text { width } \\
{[\mathrm{mm}]}\end{array}$} & 2.076 & 0.151 & 1.442 & 242 & 0.150 & 2.45177 & 1.69983 \\
\hline & & & & 1.539 & 68.204 & 0.128 & 2.45177 & 1.59334 \\
\hline & \multirow{2}{*}{$\begin{array}{l}\text { thickness } \\
{[\mathrm{mm}]}\end{array}$} & 1.243 & 0.266 & -2.834 & 242 & 0.005 & -2.22023 & 0.78330 \\
\hline & & & & -3.000 & 67.559 & 0.004 & -2.22023 & 0.73996 \\
\hline
\end{tabular}


Table 123. Ságvár flake tool length, width and thickness mean comparison by raw materials with t-test

\begin{tabular}{|l|c|c|c|c|c|c|c|}
\hline \multicolumn{1}{|c|}{} & $\begin{array}{c}\text { Levene's Test } \\
\text { for Equality } \\
\text { of Variances }\end{array}$ & \multicolumn{6}{c|}{ Independent Samples Test } \\
\cline { 2 - 8 } & $\mathrm{F}$ & Sig. & $\mathrm{t}$ & $\mathrm{df}$ & $\begin{array}{c}\text { Sig. } \\
(2 \text {-tailed })\end{array}$ & $\begin{array}{c}\text { Mean } \\
\text { difference }\end{array}$ & $\begin{array}{c}\text { Std. error } \\
\text { difference }\end{array}$ \\
\cline { 2 - 8 } & 0.113 & 0.737 & -4.257 & 164 & 0.000 & -6.29151 & 1.47797 \\
\cline { 2 - 8 } $\begin{array}{l}\text { Length } \\
{[\mathrm{mm}]}\end{array}$ & & & -4.029 & 69.053 & 0.000 & -6.29151 & 1.56170 \\
\hline $\begin{array}{l}\text { Width } \\
{[\mathrm{mm}]}\end{array}$ & 1.585 & 0.210 & -3.060 & 164 & 0.003 & -4.41986 & 1.44417 \\
\cline { 2 - 8 } $\begin{array}{l}\text { Thickness } \\
{[\mathrm{mm}]}\end{array}$ & 0.059 & 0.809 & -4.686 & 164 & 0.000 & -3.23297 & 0.68998 \\
\cline { 2 - 8 } & & & -4.363 & 67.254 & 0.000 & -3.23297 & 0.74099 \\
\hline
\end{tabular}

Table 124. Ságvár flake tool types by raw material

\begin{tabular}{|c|c|c|c|c|c|c|}
\hline & \multicolumn{3}{|c|}{ Raw material } & \multirow{2}{*}{ Total } \\
\hline & & & regional & distant & transcarpathian & \\
\hline \multirow[t]{16}{*}{ Tooltypes } & \multirow[t]{2}{*}{ endscraper } & count & 24 & 12 & 0 & 36 \\
\hline & & $\%$ within raw material & $19.7 \%$ & $27.3 \%$ & $0.0 \%$ & $21.6 \%$ \\
\hline & \multirow[t]{2}{*}{ burin } & count & 32 & 7 & 0 & 39 \\
\hline & & $\%$ within raw material & $26.2 \%$ & $15.9 \%$ & $0.0 \%$ & $23.4 \%$ \\
\hline & \multirow[t]{2}{*}{ retouched } & count & 21 & 6 & 0 & 27 \\
\hline & & $\%$ within raw material & $17.2 \%$ & $13.6 \%$ & $0.0 \%$ & $16.2 \%$ \\
\hline & \multirow[t]{2}{*}{ borer } & count & 4 & 0 & 0 & 4 \\
\hline & & $\%$ within raw material & $3.3 \%$ & $0.0 \%$ & $0.0 \%$ & $2.4 \%$ \\
\hline & \multirow{2}{*}{$\begin{array}{l}\text { splintered } \\
\text { piece }\end{array}$} & count & 27 & 11 & 1 & 39 \\
\hline & & $\%$ within raw material & $22.1 \%$ & $25.0 \%$ & $100.0 \%$ & $23.4 \%$ \\
\hline & \multirow[t]{2}{*}{ truncation } & count & 3 & 1 & 0 & 4 \\
\hline & & $\%$ within raw material & $2.5 \%$ & $2.3 \%$ & $0.0 \%$ & $2.4 \%$ \\
\hline & \multirow{2}{*}{$\begin{array}{l}\text { notched- } \\
\text { denticulated }\end{array}$} & count & 9 & 7 & 0 & 16 \\
\hline & & $\%$ within raw material & $7.4 \%$ & $15.9 \%$ & $0.0 \%$ & $9.6 \%$ \\
\hline & \multirow[t]{2}{*}{ composite } & count & 2 & 0 & 0 & 2 \\
\hline & & $\%$ within raw material & $1.6 \%$ & $0.0 \%$ & $0.0 \%$ & $1.2 \%$ \\
\hline \multirow{2}{*}{\multicolumn{2}{|c|}{ Total }} & count & 122 & 44 & 1 & 167 \\
\hline & & $\%$ within raw material & $100.0 \%$ & $100.0 \%$ & $100.0 \%$ & $100.0 \%$ \\
\hline
\end{tabular}


Lithic analysis of the Middle and Late Upper Palaeolithic in Hungary

Table 125. Ságvár debris and rejuvenating flake tool types by raw material

\begin{tabular}{|c|c|c|c|c|c|c|}
\hline \multirow{2}{*}{\multicolumn{4}{|c|}{ Debitage }} & \multicolumn{2}{|c|}{ Raw material } & \multirow{3}{*}{$\begin{array}{c}\text { Total } \\
5\end{array}$} \\
\hline & & & & \multirow{2}{*}{$\frac{\text { regional }}{4}$} & \multirow{2}{*}{$\begin{array}{c}\text { distant } \\
1\end{array}$} & \\
\hline \multirow[t]{14}{*}{ Debris } & \multirow[t]{12}{*}{ tooltypes } & \multirow[t]{2}{*}{ endscraper } & count & & & \\
\hline & & & \% within raw material & $9.5 \%$ & $7.7 \%$ & $9.1 \%$ \\
\hline & & \multirow[t]{2}{*}{ burin } & count & 10 & 2 & 12 \\
\hline & & & \% within raw material & $23.8 \%$ & $15.4 \%$ & $21.8 \%$ \\
\hline & & \multirow[t]{2}{*}{ retouched } & count & 4 & 3 & 7 \\
\hline & & & \% within raw material & $9.5 \%$ & $23.1 \%$ & $12.7 \%$ \\
\hline & & \multirow[t]{2}{*}{ borer } & count & 1 & 0 & 1 \\
\hline & & & \% within raw material & $2.4 \%$ & $0.0 \%$ & $1.8 \%$ \\
\hline & & \multirow[t]{2}{*}{ splintered piece } & count & 17 & 3 & 20 \\
\hline & & & \% within raw material & $40.5 \%$ & $23.1 \%$ & $36.4 \%$ \\
\hline & & \multirow{2}{*}{$\begin{array}{l}\text { notched- } \\
\text { denticulated }\end{array}$} & count & 6 & 4 & 10 \\
\hline & & & $\%$ within raw material & $14.3 \%$ & $30.8 \%$ & $18.2 \%$ \\
\hline & \multirow{2}{*}{\multicolumn{2}{|c|}{ total }} & count & 42 & 13 & 55 \\
\hline & & & $\%$ within raw material & $100.0 \%$ & $100.0 \%$ & $100.0 \%$ \\
\hline \multirow{10}{*}{$\begin{array}{l}\text { Rejuvenating } \\
\text { flake }\end{array}$} & \multirow[t]{8}{*}{ tooltypes } & \multirow[t]{2}{*}{ endscraper } & count & 2 & 0 & 2 \\
\hline & & & \% within raw material & $50.0 \%$ & $0.0 \%$ & $40.0 \%$ \\
\hline & & \multirow[t]{2}{*}{ burin } & count & 0 & 1 & 1 \\
\hline & & & \% within raw material & $0.0 \%$ & $100.0 \%$ & $20.0 \%$ \\
\hline & & \multirow[t]{2}{*}{ retouched } & count & 1 & 0 & 1 \\
\hline & & & \% within raw material & $25.0 \%$ & $0.0 \%$ & $20.0 \%$ \\
\hline & & \multirow[t]{2}{*}{ splintered piece } & count & 1 & 0 & 1 \\
\hline & & & \% within raw material & $25.0 \%$ & $0.0 \%$ & $20.0 \%$ \\
\hline & \multirow{2}{*}{\multicolumn{2}{|c|}{ total }} & count & 4 & 1 & 5 \\
\hline & & & \% within raw material & $100.0 \%$ & $100.0 \%$ & $100.0 \%$ \\
\hline \multirow[t]{14}{*}{ Total } & \multirow[t]{12}{*}{ tooltypes } & \multirow[t]{2}{*}{ endscraper } & count & 6 & 1 & 7 \\
\hline & & & \% within raw material & $13.0 \%$ & $7.1 \%$ & $11.7 \%$ \\
\hline & & \multirow[t]{2}{*}{ burin } & count & 10 & 3 & 13 \\
\hline & & & \% within raw material & $21.7 \%$ & $21.4 \%$ & $21.7 \%$ \\
\hline & & \multirow[t]{2}{*}{ retouched } & count & 5 & 3 & 8 \\
\hline & & & \% within raw material & $10.9 \%$ & $21.4 \%$ & $13.3 \%$ \\
\hline & & borer & count & 1 & 0 & 1 \\
\hline & & & \% within raw material & $2.2 \%$ & $0.0 \%$ & $1.7 \%$ \\
\hline & & splintered piece & count & 18 & 3 & 21 \\
\hline & & & \% within raw material & $39.1 \%$ & $21.4 \%$ & $35.0 \%$ \\
\hline & & notched- & count & 6 & 4 & 10 \\
\hline & & denticulated & \% within raw material & $13.0 \%$ & $28.6 \%$ & $16.7 \%$ \\
\hline & total & & count & 46 & 14 & 60 \\
\hline & & & \% within raw material & $100.0 \%$ & $100.0 \%$ & $100.0 \%$ \\
\hline
\end{tabular}




\section{CORVIN-TÉR}

The site

Corvin-tér is situated on the right bank of Danube in the hearth of Budapest $105 \mathrm{~m}$ a.s.l. (RINGER, LENGYEL 2008-2009). The archaeological site was found during a construction in 1997. The Upper Palaeolithic site covered a topographically tilting area $3.50-4.20 \mathrm{~m}$ below the actual surface.

All together 30 square meters were recovered from the Upper Palaeolithic settlement. The archaeological layer $1-3 \mathrm{~cm}$ thick lay above a loamy clay and under a silt loam layer. The silt loam layer $30-50 \mathrm{~cm}$ thick consisted of 2-4 cm thick laminae. This structure is characteristic to water lain sediments. The abandonment of the site was followed by water level rise of the Danube. The layer covering the archaeological remains was formed during suffusions of the Danube which lay down the fine silt loam up to $1.40 \mathrm{~m}$ thickness.

A total of $1.50 \mathrm{~m}$ thick Pleistocene sediment was studied at the site. Accordingly, changes in the sedimentation could have been observed on the interface of the loamy clay and the silt loam, where the human settlement itself was preserved.

The archaeological layer partially was reddish and dark gray. These hues in the sediment most probably were signs of fire, although hearths and charcoals were not found. The low velocity water flow of the Danube, as seen in the sediment, might have washed away the combustion features. Contrary to the charcoals, minute knapping chips, $<5 \mathrm{~mm}$, are present in the archaeological assemblage. A few organic remains were recovered at the site. Botanical remains were Pinus silvestris, including a large piece of bark. The fauna was dominated by bison.

Interesting features were footprints of animals and humans in the silt loam. A total of ten postholes were reported from the site, which ambiguously could have been dated to the human settlement.

Most of the finds are lithics. Lithic refitting study found close spatial distribution of finds. The knapped lithic artefacts lay scattered in the excavation area. A few refitted specimens lay next to each other. Animal remains were concentrated in the western, southwestern squares of the site.

\section{Raw materials}

Four types of lithic raw materials compose the assemblage: radiolarite, a dark green silicified sand stone, a dark grey silicified clay stone, and silicified lime stone (Tab. 126). By count, radiolarite is the dominant raw material (Tab. 127), which weighs $0.632 \mathrm{~kg}$. In contrast, the quartzite that counts less but weighs a few grams more (Tab. 126). The silicified limestone is also a small group of finds but its weight is heavy compared to the number of finds it yielded.

The radiolarite derived from regional sources, the Bakony Mountains, Western Hungary. Its colour is brown, reddish and yellowish, which is typical for that area. 
The silicified materials are of yet unknown origin, but their pebble cortex may refer to that they have been collected from the locally available Danube gravel. Local material yielded 60.13 items per $\mathrm{kg}$, while the regional did 295.1.

\section{Blade tool production}

The distribution of the technological categories between the regional and the local materials is different. Regional materials yielded all blade technological categories, while the local materials were used for making flakes (Tab. 127).

Blades were unidirectionally detached (Tab. 128) and out of the four blade cores three have single striking platform. The blades have plain platforms (Tab. 129) with impact points missing and overhangs abraded (Tab. 130) showing soft hammer percussion. The blades are regularly short (Tab. 131) and the blade cores are also small sized (Tab. 132).

All the tools were made of the regional material, and $53.8 \%$ of the tools were made of blades. A total of $10.2 \%$ of the flakes are tools while this percent is 14.1 for the blades.

The mean length of the blade tools also is short (Tab. 133), even shorter than the blank items, however, this difference is rather insignificant (Tab. 134).

Blade tools are mostly edge retouched types (Tab. 135) and the few armatures are three backed bladelets.

\section{Flake tool production}

Unidirectional flake debitage characterizes the local material processing (Tab. 136). Flake platforms are chiefly cortical in the local group or plain, while regional specimens are plain (Tab. 137). Local and regional flakes bear no impact point but unabraded overhangs as a result of hard hammer percussion (Tab. 138). However, overhang abrasion on the regional flakes shows minor use of soft hammer percussion, too. Regional flakes are smaller than local ones (Tab. 139), and this difference is significant (Tab. 140).

Flake tools tend to be longer and thicker than the blank flakes (Tab. 141) and this difference is insignificant (Tab. 142). Flake tools are end scrapers, burins, edge retouched items and notches-denticulates (Tab. 143). 
Table 126. Corvin-tér lithic raw material composition by weight in grams

\begin{tabular}{|l|c|c|c|c|c|c|c|}
\hline Raw material & Blade & Flake & Debris & Blade core & Flake core & Total & $\%$ \\
\hline Regional & 145.38 & 318.67 & 116.9 & 52.02 & & 632.97 & 36.00 \\
\hline Within regional \% & 22.96 & 50.34 & 18.46 & 8.21 & & 100 & \\
\hline Local & & 306.81 & 406.01 & & 412.2 & 1125.02 & 63.99 \\
\hline Within local \% & & 47.58 & 62.975 & & 63.93 & 100 & \\
\hline Total & 145.38 & 625.48 & 522.91 & 52.02 & 412.2 & 1757.99 & 100 \\
\hline$\%$ & 8.26 & 35.57 & 29.74 & 2.95 & 23.44 & 100 & \\
\hline
\end{tabular}

Table 127. Corvin-tér lithic assemblage composition by raw material types and technological categories

\begin{tabular}{|c|c|c|c|c|c|}
\hline & \multicolumn{2}{|c|}{ Raw material } & \multirow{2}{*}{ Total } \\
\hline & & & local & regional & \\
\hline \multirow[t]{14}{*}{ Class } & \multirow[t]{2}{*}{ flake } & count & 27 & 118 & 145 \\
\hline & & $\%$ within raw material & $71.1 \%$ & $35.5 \%$ & $39.2 \%$ \\
\hline & \multirow[t]{2}{*}{ blade } & count & 0 & 93 & 93 \\
\hline & & $\%$ within raw material & $0.0 \%$ & $28.0 \%$ & $25.1 \%$ \\
\hline & \multirow[t]{2}{*}{ debris } & count & 10 & 105 & 115 \\
\hline & & $\%$ within raw material & $26.3 \%$ & $31.6 \%$ & $31.1 \%$ \\
\hline & \multirow{2}{*}{$\begin{array}{l}\text { rejuvenating } \\
\text { flake }\end{array}$} & count & 0 & 6 & 6 \\
\hline & & $\%$ within raw material & $0.0 \%$ & $1.8 \%$ & $1.6 \%$ \\
\hline & \multirow[t]{2}{*}{ neo-crest } & count & 0 & 6 & 6 \\
\hline & & $\%$ within raw material & $0.0 \%$ & $1.8 \%$ & $1.6 \%$ \\
\hline & \multirow[t]{2}{*}{ blade core } & count & 0 & 4 & 4 \\
\hline & & $\%$ within raw material & $0.0 \%$ & $1.2 \%$ & $1.1 \%$ \\
\hline & \multirow[t]{2}{*}{ flake core } & count & 1 & 0 & 1 \\
\hline & & $\%$ within raw material & $2.6 \%$ & $0.0 \%$ & $0.3 \%$ \\
\hline \multirow{2}{*}{\multicolumn{2}{|c|}{ Total }} & count & 38 & 332 & 370 \\
\hline & & $\%$ within raw material & $100.0 \%$ & $100.0 \%$ & $100.0 \%$ \\
\hline
\end{tabular}

Table 128. Corvin-tér blade assemblage (complete specimens) dorsal scar pattern frequency by raw material

\begin{tabular}{|c|c|c|c|c|}
\hline & Raw material & \multirow{2}{*}{ Total } \\
\hline & & & regional & \\
\hline \multirow[t]{8}{*}{ Scars } & \multirow[t]{2}{*}{ unidirectional } & count & 17 & 17 \\
\hline & & $\%$ within raw material & $47.2 \%$ & $47.2 \%$ \\
\hline & \multirow[t]{2}{*}{ opposite } & count & 10 & 10 \\
\hline & & $\%$ within raw material & $27.8 \%$ & $27.8 \%$ \\
\hline & \multirow[t]{2}{*}{ perpendicular } & count & 7 & 7 \\
\hline & & $\%$ within raw material & $19.4 \%$ & $19.4 \%$ \\
\hline & \multirow[t]{2}{*}{ multiple } & count & 2 & 2 \\
\hline & & $\%$ within raw material & $5.6 \%$ & $5.6 \%$ \\
\hline \multirow{2}{*}{\multicolumn{2}{|c|}{ Total }} & count & 36 & 36 \\
\hline & & $\%$ within raw material & $100.0 \%$ & $100.0 \%$ \\
\hline
\end{tabular}


Table 129. Corvin-tér blade assemblage (complete specimens) platform type frequency by raw material

\begin{tabular}{|c|c|c|c|c|}
\hline & Raw material & \multirow{2}{*}{ Total } \\
\hline & & & regional & \\
\hline \multirow[t]{10}{*}{ Platform } & \multirow[t]{2}{*}{ plain } & count & 27 & 27 \\
\hline & & $\%$ within raw material & $75.0 \%$ & $75.0 \%$ \\
\hline & \multirow[t]{2}{*}{ dihedral } & count & 2 & 2 \\
\hline & & $\%$ within raw material & $5.6 \%$ & $5.6 \%$ \\
\hline & \multirow[t]{2}{*}{ faceted } & count & 5 & 5 \\
\hline & & $\%$ within raw material & $13.9 \%$ & $13.9 \%$ \\
\hline & \multirow[t]{2}{*}{ linear } & count & 1 & 1 \\
\hline & & $\%$ within raw material & $2.8 \%$ & $2.8 \%$ \\
\hline & \multirow[t]{2}{*}{ irregular } & count & 1 & 1 \\
\hline & & $\%$ within raw material & $2.8 \%$ & $2.8 \%$ \\
\hline \multirow{2}{*}{\multicolumn{2}{|c|}{ Total }} & count & 36 & 36 \\
\hline & & $\%$ within raw material & $100.0 \%$ & $100.0 \%$ \\
\hline
\end{tabular}

Table 130. Corvin-tér blade assemblage (complete specimens) impact point-overhang co-presence by raw material

\begin{tabular}{|l|l|l|c|c|}
\hline \multicolumn{2}{|c|}{} & Raw material & \multirow{2}{*}{ Total } \\
\cline { 3 - 5 } $\begin{array}{l}\text { Impact } \\
\text { point-overhang }\end{array}$ & none & count & 20 & 20 \\
\cline { 3 - 5 } & & $\%$ within raw material & $55.6 \%$ & $55.6 \%$ \\
\cline { 2 - 5 } & \multirow{2}{*}{ yes-no } & count & 4 & 4 \\
\cline { 3 - 5 } & \multirow{3}{*}{ yes-yes } & \% within raw material & $11.1 \%$ & $11.1 \%$ \\
\cline { 3 - 5 } & & $\%$ within raw material & $8.3 \%$ & $8.3 \%$ \\
\cline { 3 - 5 } & no-yes & count & 9 & 9 \\
\cline { 3 - 5 } & \% within raw material & $25.0 \%$ & $25.0 \%$ \\
\hline \multirow{2}{*}{ Total } & count & 36 & 36 \\
\cline { 3 - 5 } & \% within raw material & $100.0 \%$ & $100.0 \%$ \\
\hline
\end{tabular}

Table 131. Corvin-tér blade assemblage (complete specimens) length, width and thickness by raw material

\begin{tabular}{|l|l|c|c|c|}
\hline Raw material & & Length [mm] & Width [mm] & Thickness [mm] \\
\hline Regional & Minimum & 16.50 & 7.60 & 1.40 \\
\cline { 2 - 5 } & Maximum & 45.00 & 17.60 & 11.20 \\
\cline { 2 - 5 } & Mean & 28.4833 & 11.7333 & 4.9806 \\
\cline { 2 - 5 } & Median & 27.9500 & 11.2000 & 4.8000 \\
\cline { 2 - 5 } & N & 36 & 36 & 36 \\
\cline { 2 - 5 } & Std. deviation & 7.34534 & 3.09442 & 2.37785 \\
\hline \multirow{5}{*}{ Total } & Minimum & 16.50 & 7.60 & 1.40 \\
\cline { 2 - 5 } & Maximum & 45.00 & 17.60 & 11.20 \\
\cline { 2 - 5 } & Mean & 28.4833 & 11.7333 & 4.9806 \\
\cline { 2 - 5 } & Median & 27.9500 & 11.2000 & 3.8000 \\
\cline { 2 - 5 } & N & 36 & 36 & 2.37785 \\
\cline { 2 - 5 } & Std. deviation & 7.34534 & 3.09442 & \\
\hline
\end{tabular}


Table 132. Corvin-tér core size

\begin{tabular}{|l|l|c|c|c|}
\hline Debitage & Length & Width & Depth \\
\hline Flake & Minimum & 37.70 & 111.50 & 67.96 \\
\cline { 2 - 5 } & Maximum & 37.70 & 111.50 & 67.96 \\
\cline { 2 - 5 } & Mean & 37.7000 & 111.5000 & 67.9600 \\
\cline { 2 - 5 } & Median & 37.7000 & 111.5000 & 67.9600 \\
\cline { 2 - 5 } & N & 1 & 1 & 1 \\
\cline { 2 - 5 } & Std. deviation & - & - & - \\
\hline \multirow{5}{*}{ Blade } & Minimum & 27.00 & 18.77 & 9.80 \\
\cline { 2 - 5 } & Maximum & 31.30 & 28.50 & 30.38 \\
\cline { 2 - 5 } & Mean & 28.9600 & 21.3775 & 19.7650 \\
\cline { 2 - 5 } & Median & 28.7700 & 19.1200 & 4 \\
\cline { 2 - 5 } & N & 4 & 4 & 11.33641 \\
\cline { 2 - 5 } & Std. deviation & 1.79473 & 4.75347 & \\
\hline
\end{tabular}

Table 133. Corvin-tér blade assemblage (complete specimens) length, width and thickness by raw material

\begin{tabular}{|l|l|c|c|c|}
\hline Raw material & Length [mm] & Width [mm] & Thickness [mm] \\
\hline \multirow{5}{*}{ Regional } & Minimum & 15.50 & 6.00 & 1.50 \\
\cline { 2 - 5 } & Maximum & 35.70 & 23.20 & 17.00 \\
\cline { 2 - 5 } & Mean & 25.7286 & 11.2857 & 4.4714 \\
\cline { 2 - 5 } & Median & 25.4500 & 10.0500 & 3.1000 \\
\cline { 2 - 5 } & N & 14 & 14 & 14 \\
\cline { 2 - 5 } & Std. deviation & 6.31925 & 4.81278 & 4.01467 \\
\hline \multirow{5}{*}{ Total } & Minimum & 15.50 & 6.00 & 1.50 \\
\cline { 2 - 5 } & Maximum & 35.70 & 23.20 & 17.00 \\
\cline { 2 - 5 } & Mean & 25.7286 & 11.2857 & 4.4714 \\
\cline { 2 - 5 } & Median & 25.4500 & 10.0500 & 3.1000 \\
\cline { 2 - 5 } & N & 14 & 14 & 4.01467 \\
\cline { 2 - 5 } & Std. deviation & 6.31925 & 4.81278 & \\
\hline
\end{tabular}

Table 134. Corvin-tér blade blank and tool length, width and thickness mean t-test comparison

\begin{tabular}{|c|c|c|c|c|c|c|c|}
\hline \multicolumn{8}{|c|}{ Independent Samples Test } \\
\hline & \multicolumn{2}{|c|}{$\begin{array}{l}\text { Levene's Test } \\
\text { for Equality } \\
\text { of Variances }\end{array}$} & \multicolumn{5}{|c|}{ t-test for Equality of Means } \\
\hline & $\mathrm{F}$ & Sig. & $\mathrm{t}$ & df & $\begin{array}{c}\text { Sig. } \\
\text { (2-tailed) }\end{array}$ & $\begin{array}{c}\text { Mean } \\
\text { difference }\end{array}$ & $\begin{array}{l}\text { Std. error } \\
\text { difference }\end{array}$ \\
\hline \multirow{2}{*}{$\begin{array}{l}\text { Length } \\
{[\mathrm{mm}]}\end{array}$} & 0.422 & 0.519 & 1.235 & 48 & 0.223 & 2.75476 & 2.23066 \\
\hline & & & 1.321 & 27.437 & 0.198 & 2.75476 & 2.08592 \\
\hline \multirow{2}{*}{$\begin{array}{l}\text { Width } \\
{[\mathrm{mm}]}\end{array}$} & 3.258 & 0.077 & 0.390 & 48 & 0.698 & 0.44762 & 1.14674 \\
\hline & & & 0.323 & 17.349 & 0.751 & 0.44762 & 1.38581 \\
\hline \multirow{2}{*}{$\begin{array}{l}\text { Thickness } \\
{[\mathrm{mm}]}\end{array}$} & 1.490 & 0.228 & 0.555 & 48 & 0.582 & 0.50913 & 0.91764 \\
\hline & & & 0.445 & 16.674 & 0.662 & 0.50913 & 1.14382 \\
\hline
\end{tabular}


Table 135. Corvin-tér blade tool types by raw material

\begin{tabular}{|c|c|c|c|c|}
\hline & Raw Material & \multirow{2}{*}{ Total } \\
\hline & & & regional & \\
\hline \multirow[t]{12}{*}{ Tooltypes } & \multirow[t]{2}{*}{ burin } & count & 1 & 1 \\
\hline & & \% within raw material & $7.1 \%$ & $7.1 \%$ \\
\hline & \multirow[t]{2}{*}{ retouched } & count & 5 & 5 \\
\hline & & $\%$ within raw material & $35.7 \%$ & $35.7 \%$ \\
\hline & \multirow[t]{2}{*}{ borer } & count & 1 & 1 \\
\hline & & $\%$ within raw material & $7.1 \%$ & $7.1 \%$ \\
\hline & \multirow[t]{2}{*}{ truncation } & count & 3 & 3 \\
\hline & & $\%$ within raw material & $21.4 \%$ & $21.4 \%$ \\
\hline & \multirow[t]{2}{*}{ composite } & count & 1 & 1 \\
\hline & & $\%$ within raw material & $7.1 \%$ & $7.1 \%$ \\
\hline & \multirow[t]{2}{*}{ armature } & count & 3 & 3 \\
\hline & & $\%$ within raw material & $21.4 \%$ & $21.4 \%$ \\
\hline \multirow{2}{*}{\multicolumn{2}{|c|}{ Total }} & count & 14 & 14 \\
\hline & & $\%$ within raw material & $100.0 \%$ & $100.0 \%$ \\
\hline
\end{tabular}

Table 136. Corvin-tér flake assemblage (complete specimens) dorsal scar pattern frequency by raw material

\begin{tabular}{|c|c|c|c|c|c|}
\hline & \multicolumn{2}{|c|}{ Raw material } & \multirow{2}{*}{ Total } \\
\hline & & & local & regional & \\
\hline \multirow[t]{10}{*}{ Scars } & \multirow[t]{2}{*}{ unidirectional } & count & 6 & 18 & 24 \\
\hline & & $\%$ within raw material & $42.9 \%$ & $36.7 \%$ & $38.1 \%$ \\
\hline & \multirow{2}{*}{ opposite } & count & 1 & 6 & 7 \\
\hline & & $\%$ within raw material & $7.1 \%$ & $12.2 \%$ & $11.1 \%$ \\
\hline & \multirow[t]{2}{*}{ perpendicular } & count & 4 & 16 & 20 \\
\hline & & $\%$ within raw material & $28.6 \%$ & $32.7 \%$ & $31.7 \%$ \\
\hline & \multirow[t]{2}{*}{ multiple } & count & 1 & 9 & 10 \\
\hline & & $\%$ within raw material & $7.1 \%$ & $18.4 \%$ & $15.9 \%$ \\
\hline & \multirow[t]{2}{*}{ no scar } & count & 2 & 0 & 2 \\
\hline & & $\%$ within raw material & $14.3 \%$ & $0.0 \%$ & $3.2 \%$ \\
\hline \multirow{2}{*}{\multicolumn{2}{|c|}{ Total }} & count & 14 & 49 & 63 \\
\hline & & $\%$ within raw material & $100.0 \%$ & $100.0 \%$ & $100.0 \%$ \\
\hline
\end{tabular}

Table 137. Corvin-tér flake assemblage (complete specimens) platform type frequency by raw material

\begin{tabular}{|c|c|c|c|c|c|}
\hline & \multicolumn{2}{|c|}{ Raw material } & \multirow{2}{*}{ Total } \\
\hline & & & local & regional & \\
\hline \multirow[t]{10}{*}{ Platform } & \multirow[t]{2}{*}{ plain } & count & 3 & 32 & 35 \\
\hline & & $\%$ within raw material & $21.4 \%$ & $65.3 \%$ & $55.6 \%$ \\
\hline & \multirow[t]{2}{*}{ dihedral } & count & 1 & 6 & 7 \\
\hline & & $\%$ within raw material & $7.1 \%$ & $12.2 \%$ & $11.1 \%$ \\
\hline & \multirow[t]{2}{*}{ faceted } & count & 0 & 9 & 9 \\
\hline & & $\%$ within raw material & $0.0 \%$ & $18.4 \%$ & $14.3 \%$ \\
\hline & \multirow[t]{2}{*}{ cortical } & count & 10 & 0 & 10 \\
\hline & & $\%$ within raw material & $71.4 \%$ & $0.0 \%$ & $15.9 \%$ \\
\hline & \multirow[t]{2}{*}{ irregular } & count & 0 & 2 & 2 \\
\hline & & $\%$ within raw material & $0.0 \%$ & $4.1 \%$ & $3.2 \%$ \\
\hline \multirow{2}{*}{\multicolumn{2}{|c|}{ Total }} & count & 14 & 49 & 63 \\
\hline & & $\%$ within raw material & $100.0 \%$ & $100.0 \%$ & $100.0 \%$ \\
\hline
\end{tabular}


Table 138. Corvin-tér flake assemblage (complete specimens) impact point-overhang frequency by raw material

\begin{tabular}{|c|c|c|c|c|c|}
\hline & \multicolumn{2}{|c|}{ Raw material } & \multirow[b]{2}{*}{ Total } \\
\hline & & & local & regional & \\
\hline \multirow{8}{*}{$\begin{array}{l}\text { Impact } \\
\text { point-overhang }\end{array}$} & \multirow[t]{2}{*}{ none } & count & 0 & 11 & 11 \\
\hline & & $\%$ within raw material & $0.0 \%$ & $22.4 \%$ & $17.5 \%$ \\
\hline & \multirow[t]{2}{*}{ yes-no } & count & 0 & 11 & 11 \\
\hline & & $\%$ within raw material & $0.0 \%$ & $22.4 \%$ & $17.5 \%$ \\
\hline & \multirow[t]{2}{*}{ yes-yes } & count & 3 & 10 & 13 \\
\hline & & \% within raw material & $21.4 \%$ & $20.4 \%$ & $20.6 \%$ \\
\hline & \multirow[t]{2}{*}{ no-yes } & count & 11 & 17 & 28 \\
\hline & & \% within raw material & $78.6 \%$ & $34.7 \%$ & $44.4 \%$ \\
\hline \multirow{2}{*}{\multicolumn{2}{|c|}{ Total }} & count & 14 & 49 & 63 \\
\hline & & \% within raw material & $100.0 \%$ & $100.0 \%$ & $100.0 \%$ \\
\hline
\end{tabular}

Table 139. Corvin-tér flake blank assemblage length, width and thickness by raw materials

\begin{tabular}{|c|c|c|c|c|}
\hline \multicolumn{2}{|c|}{ Raw material } & Length $[\mathrm{mm}]$ & Width [mm] & Thickness [mm] \\
\hline \multirow[t]{6}{*}{ Local } & Minimum & 19.10 & 17.90 & 4.50 \\
\hline & Maximum & 76.30 & 76.10 & 27.00 \\
\hline & Median & 36.0500 & 32.8000 & 9.4000 \\
\hline & Mean & 40.7571 & 38.4143 & 11.0500 \\
\hline & $\mathrm{N}$ & 14 & 14 & 14 \\
\hline & Std. deviation & 16.97599 & 17.66421 & 6.91640 \\
\hline \multirow[t]{6}{*}{ Regional } & Minimum & 10.60 & 7.30 & 1.30 \\
\hline & Maximum & 35.90 & 34.00 & 15.90 \\
\hline & Median & 23.4000 & 16.2000 & 5.5000 \\
\hline & Mean & 22.4959 & 16.7204 & 5.9163 \\
\hline & $\mathrm{N}$ & 49 & 49 & 49 \\
\hline & Std. deviation & 6.75811 & 5.39274 & 3.10922 \\
\hline \multirow[t]{6}{*}{ Total } & Minimum & 10.60 & 7.30 & 1.30 \\
\hline & Maximum & 76.30 & 76.10 & 27.00 \\
\hline & Median & 24.5000 & 17.9000 & 6.1000 \\
\hline & Mean & 26.5540 & 21.5413 & 7.0571 \\
\hline & $\mathrm{N}$ & 63 & 63 & 63 \\
\hline & Std. deviation & 12.42383 & 13.06115 & 4.70565 \\
\hline
\end{tabular}

Table 140. Corvin-tér flake blank length, width and thickness mean comparison by raw materials with t-test

\begin{tabular}{|c|c|c|c|c|c|c|c|}
\hline \multicolumn{8}{|c|}{ Independent Samples Test } \\
\hline & \multicolumn{2}{|c|}{$\begin{array}{l}\text { Levene's Test } \\
\text { for Equality } \\
\text { of Variances }\end{array}$} & \multicolumn{5}{|c|}{ t-test for Equality of Means } \\
\hline & $\mathrm{F}$ & Sig. & $\mathrm{t}$ & df & Sig. (2-tailed) & $\begin{array}{c}\text { Mean } \\
\text { difference }\end{array}$ & $\begin{array}{l}\text { Std. error } \\
\text { difference }\end{array}$ \\
\hline \multirow{2}{*}{$\begin{array}{l}\text { Length } \\
{[\mathrm{mm}]}\end{array}$} & 26.312 & 0.000 & 6.107 & 61 & 0.000 & 18.26122 & 2.99011 \\
\hline & & & 3.937 & 14.196 & 0.001 & 18.26122 & 4.63861 \\
\hline \multirow{2}{*}{$\begin{array}{l}\text { Width } \\
{[\mathrm{mm}]}\end{array}$} & 39.785 & 0.000 & 7.572 & 61 & 0.000 & 21.69388 & 2.86504 \\
\hline & & & 4.535 & 13.699 & 0.000 & 21.69388 & 4.78340 \\
\hline \multirow{2}{*}{$\begin{array}{l}\text { Thickness } \\
{[\mathrm{mm}]}\end{array}$} & 9.956 & 0.002 & 4.015 & 61 & 0.000 & 5.13367 & 1.27861 \\
\hline & & & 2.700 & 14.531 & 0.017 & 5.13367 & 1.90110 \\
\hline
\end{tabular}


Lithic analysis of the Middle and Late Upper Palaeolithic in Hungary

Table 141. Corvin-tér flake tool assemblage length, width and thickness by raw materials

\begin{tabular}{|l|l|c|c|c|}
\hline Raw material & & Length [mm] & Width [mm] & Thickness [mm] \\
\hline Regional & Minimum & 12.10 & 13.20 & 3.30 \\
\cline { 2 - 5 } & Maximum & 51.00 & 34.00 & 18.10 \\
\cline { 2 - 5 } & Mean & 27.7167 & 20.6500 & 8.8667 \\
\cline { 2 - 5 } & Median & 26.8500 & 18.6000 & 8.5500 \\
\cline { 2 - 5 } & N & 12 & 12 & 12 \\
\cline { 2 - 5 } & Std. deviation & 9.48260 & 6.01899 & 3.66193 \\
\hline \multirow{5}{*}{ Total } & Minimum & 12.10 & 13.20 & 3.30 \\
\cline { 2 - 5 } & Maximum & 51.00 & 34.00 & 18.10 \\
\cline { 2 - 5 } & Mean & 27.7167 & 20.6500 & 8.8667 \\
\cline { 2 - 5 } & Median & 26.8500 & 18.6000 & 12 \\
\cline { 2 - 5 } & N & 12 & 12 & 3.66193 \\
\cline { 2 - 5 } & Std. deviation & 9.48260 & 6.01899 & \\
\hline
\end{tabular}

Table 142. Corvin-tér flake blank and tool length, width and thickness mean t-test comparison by raw material

\begin{tabular}{|c|c|c|c|c|c|c|c|}
\hline \multicolumn{8}{|c|}{ Independent Samples Test } \\
\hline & \multicolumn{2}{|c|}{$\begin{array}{l}\text { Levene's Test } \\
\text { for Equality } \\
\text { of Variances }\end{array}$} & \multicolumn{5}{|c|}{ t-test for Equality of Means } \\
\hline & $\mathrm{F}$ & Sig. & $\mathrm{t}$ & df & Sig. (2-tailed) & $\begin{array}{c}\text { Mean } \\
\text { difference }\end{array}$ & $\begin{array}{l}\text { Std. error } \\
\text { difference }\end{array}$ \\
\hline \multirow{2}{*}{$\begin{array}{l}\text { Length } \\
{[\mathrm{mm}]}\end{array}$} & 0.662 & 0.418 & -0.307 & 73 & 0.760 & -1.16270 & 3.78807 \\
\hline & & & -0.369 & 19.009 & 0.716 & -1.16270 & 3.15331 \\
\hline \multirow{2}{*}{$\begin{array}{l}\text { Width } \\
{[\mathrm{mm}]}\end{array}$} & 1.971 & 0.165 & 0.231 & 73 & 0.818 & 0.89127 & 3.86204 \\
\hline & & & 0.372 & 34.638 & 0.712 & 0.89127 & 2.39308 \\
\hline \multirow{2}{*}{$\begin{array}{l}\text { Thickness } \\
{[\mathrm{mm}]}\end{array}$} & 0.517 & 0.475 & -1.259 & 73 & 0.212 & -1.80952 & 1.43742 \\
\hline & & & -1.493 & 18.680 & 0.152 & -1.80952 & 1.21200 \\
\hline
\end{tabular}

Table 143. Corvin-tér flake tool types by raw material

\begin{tabular}{|c|c|c|c|c|}
\hline & \multirow{2}{*}{$\begin{array}{c}\text { Raw material } \\
\text { regional }\end{array}$} & \multirow[b]{2}{*}{ Total } \\
\hline & & & & \\
\hline \multirow[t]{8}{*}{ Tooltypes } & \multirow[t]{2}{*}{ endscraper } & count & 5 & 5 \\
\hline & & $\%$ within raw material & $41.7 \%$ & $41.7 \%$ \\
\hline & \multirow[t]{2}{*}{ burin } & count & 3 & 3 \\
\hline & & $\%$ within raw material & $25.0 \%$ & $25.0 \%$ \\
\hline & \multirow[t]{2}{*}{ retouched } & count & 2 & 2 \\
\hline & & $\%$ within raw material & $16.7 \%$ & $16.7 \%$ \\
\hline & \multirow{2}{*}{\begin{tabular}{|l} 
notched- \\
denticulated
\end{tabular}} & count & 2 & 2 \\
\hline & & $\%$ within raw material & $16.7 \%$ & $16.7 \%$ \\
\hline \multirow{2}{*}{\multicolumn{2}{|c|}{ Total }} & count & 12 & 12 \\
\hline & & $\%$ within raw material & $100.0 \%$ & $100.0 \%$ \\
\hline
\end{tabular}




\section{ESZTERGOM}

The site

The site is located in North Hungary, the western edge of Visegrád Mountains, on the right bank of Danube, northeast to the core of town Esztergom (DoBosi, KöveCSESVARGA 1991). This is a plateau at $160 \mathrm{~m}$ a.s.l. slightly sloping towards north under Sípoló hill $317 \mathrm{~m}$ a.s.l. An excavation in 1984 uncovered an area $\sim 145$ square meters, which yielded $\sim 1200$ archaeological finds.

The stratigraphy consisted of the recent soil on top, then a loess layer, in which the archaeological layer occurred $100 \mathrm{~cm}$ below the ground surface. Beneath the loess was the andesite bedrock. In the center of the site area was a hearth. The archaeological layer was reddish brown and contained charcoal grains and small ochre lumps. Besides this layer, finds occurred in the pure loess, too. The fauna is dominated by horse and rein deer, and also mammoth bones were found. The fauna was very fragmented and poorly preserved.

The find assemblage consisted of lithic artifacts, ochre lumps, shell ornaments and animal bones. A remarkable feature of the lithic assemblage of Gyurgyalag is the frequency of exotic raw material originating from the Prut river valley (VARGA 1991), which counts over a thousand pieces (DoBOSI, KöVECSES-VARGA 1991) making up over $90 \%$ of the total lithic assemblage. Esztergom has long been a lonely assemblage in the Hungarian archaeological record due to its lithic character. This site represented alone the second phase of the Epigravettian what Doвоsi (2004) named Epigravettian rich in backed blades. Recently, Nadap was found to be closely similar by lithic tool typology (Lengyel 2016). Charcoals from one of the hearths were dated to $16160 \pm 200 \mathrm{BP}$ (Deb-1160) (HERTELENDI 1991).

\section{Raw materials}

Transcarpathian material dominate the assemblage (Tab. 144). According to chemical analyses (VARGA 1991), these originate east of the Carpathians where the Prut cuts through the Podolian upland. However, macroscopically these flints are more similar to those found further east near the Dniester called Volhynian flint. A few items among the flints with brown hue are similar to Jurassic flint of the Kraków-Częstochowa upland, especially the Wołowice type. Transcarpathian materials yielded 431 item per $\mathrm{kg}$.

Regional materials consist of a few items of a brown limnic silicite, the origin of which could have been the Börzsöny Mountains, and radiolarite, most probably of Gerecse origin. Regional materials yielded 109.6 items per kg.

A single piece of obsidian is the distant lithic raw material. No local raw materials were identified in the assemblage. 


\section{Blade tool production}

Except the obsidian, all materials yielded complete sets of operational sequences and the frequency of blades suggests that blade debitage was the prime method to obtain blanks (Tab. 145).

Unidirectional reduction characterizes the blades (Tab. 146). Two blade cores, one made of cretaceous flint and the other made of a regional limnic silicite, also have single striking platform. Plain platforms dominate the blades (Tab. 147) and there are evidences for soft hammer percussion (Tab. 148). Transcarpathian blades are the longest items among blank blades (Tab. 149), but the t-test found no difference between the mean values of the blades by raw materials (Tab. 150).

The greatest percent of the tools are blades (Tab. 151) and out of the total number of blades more than $50 \%$ are tools (Tab. 152). The blade selection among flint blades was oriented toward the finer specimens (Tab. 153). T-test (Tab. 154) found the mean length of tool blades shorter than blank ones, which is due to that most of the blade tools are broken. The mean widths and thicknesses are not different. The other raw materials yielded insufficient number of specimens to be compared within groups. Comparing the sizes of the blade tools between raw materials (regional and transcarpathian), the t-test (Tab. 155), again, found no differences in the means.

Most of the blade tools are armatures (Tab. 156), dominated by backed bladelets, backed-truncated types, and points (Tab. 157). A few specimens have geometric shapes such as the trapeze-rectangle and the trapeze. The regional armatures are backed bladelets and one item is a backed-truncated type. The variability of armature types in the transcarpathian assemblage is greater, and all the points and geometric items belong here (Tab. 158). The majority of the points are curved backed points. There is a single specimen that has an inverse though not flat basal retouch, classified here eventually as a Gravette point. Burins were made only of transcarpathian blades.

\section{Flake tool production}

The flakes have dorsal scar orientation patterning from more than one direction (Tab. 159). This could be related with blade core shaping. The flakes have plain platforms (Tab. 160), overhangs and impact points as a result of hard hammer percussion besides the soft hammer percussion (Tab. 161).

Among blank specimens, the limnic silicite yielded the largest items (Tab. 162), and the t-test showed this difference significant (Tab. 163).

The flakes were rarely blanks of tools (Tabs 151 and 152). Only the transcarpathian material was used to make flake tools. Only $11.7 \%$ of the flakes are tools, which are rather short (Tab. 164). The t-test (Tab. 165) found no difference in mean length between tools and blank specimens of flakes concerning either the length, the width or the thickness.

The flakes are habitually edge retouched items (Tab. 166), however they also take a minor part of the armatures with a backed tool and a rectangle (Tab. 167). Although the number of burins is low, there is a considerable amount of burin spalls among the waste material. 
Table 144. Esztergom lithic raw material composition by weight in grams

\begin{tabular}{|l|c|c|c|c|c|}
\hline Raw material & Blade & Flake & Core & Total & $\%$ \\
\hline Regional & 44 & 494 & 55 & 593 & 20.25 \\
\hline Within regional \% & 7.419899 & 83.30523 & 9.274874 & 100 & \\
\hline Distant & 2 & & & 2 & 0.06 \\
\hline Within distant \% & 100 & & & 100 & \\
\hline Transcarpathian & 1667 & 626 & 41 & 2334 & 79.69 \\
\hline Within transcarpathian & 71.42245 & 26.82091 & 1.756641 & 100 & \\
\hline Total & 1713 & 1120 & 96 & 2929 & 100 \\
\hline$\%$ & 58.48412 & 38.23831 & 3.277569 & 100 & \\
\hline
\end{tabular}

Table 145. Esztergom lithic assemblage composition by raw material types and technological categories

\begin{tabular}{|c|c|c|c|c|c|c|}
\hline & \multicolumn{3}{|c|}{ Raw material } & \multirow{2}{*}{ Total } \\
\hline & & & regional & distant & transcarpathian & \\
\hline \multirow[t]{12}{*}{ Class } & \multirow[t]{2}{*}{ flake } & count & 34 & 0 & 189 & 223 \\
\hline & & $\%$ within raw material & $52.3 \%$ & $0.0 \%$ & $18.8 \%$ & $20.8 \%$ \\
\hline & \multirow[t]{2}{*}{ blade } & count & 19 & 1 & 515 & 535 \\
\hline & & $\%$ within raw material & $29.2 \%$ & $100.0 \%$ & $51.2 \%$ & $49.9 \%$ \\
\hline & \multirow[t]{2}{*}{ debris } & count & 11 & 0 & 282 & 293 \\
\hline & & $\%$ within raw material & $16.9 \%$ & $0.0 \%$ & $28.0 \%$ & $27.3 \%$ \\
\hline & \multirow{2}{*}{$\begin{array}{l}\text { rejuvenating } \\
\text { flake }\end{array}$} & count & 0 & 0 & 10 & 10 \\
\hline & & $\%$ within raw material & $0.0 \%$ & $0.0 \%$ & $1.0 \%$ & $0.9 \%$ \\
\hline & \multirow[t]{2}{*}{ neo-crest } & count & 0 & 0 & 9 & 9 \\
\hline & & $\%$ within raw material & $0.0 \%$ & $0.0 \%$ & $0.9 \%$ & $0.8 \%$ \\
\hline & \multirow[t]{2}{*}{ blade core } & count & 1 & 0 & 1 & 2 \\
\hline & & $\%$ within raw material & $1.5 \%$ & $0.0 \%$ & $0.1 \%$ & $0.2 \%$ \\
\hline \multirow{2}{*}{\multicolumn{2}{|c|}{ Total }} & count & 65 & 1 & 1006 & 1072 \\
\hline & & $\%$ within raw material & $100.0 \%$ & $100.0 \%$ & $100.0 \%$ & $100.0 \%$ \\
\hline
\end{tabular}

Table 146. Esztergom blade assemblage (complete specimens) dorsal scar pattern frequency by raw material

\begin{tabular}{|c|c|c|c|c|c|c|}
\hline & \multicolumn{3}{|c|}{ Raw material } & \multirow{2}{*}{ Total } \\
\hline & & & regional & distant & transcarpathian & \\
\hline \multirow[t]{8}{*}{ Scars } & \multirow[t]{2}{*}{ unidirectional } & count & 1 & 0 & 14 & 15 \\
\hline & & $\%$ within raw material & $50.0 \%$ & $0.0 \%$ & $63.6 \%$ & $60.0 \%$ \\
\hline & \multirow[t]{2}{*}{ opposite } & count & 1 & 1 & 3 & 5 \\
\hline & & $\%$ within raw material & $50.0 \%$ & $100.0 \%$ & $13.6 \%$ & $20.0 \%$ \\
\hline & \multirow[t]{2}{*}{ perpendicular } & count & 0 & 0 & 4 & 4 \\
\hline & & $\%$ within raw material & $0.0 \%$ & $0.0 \%$ & $18.2 \%$ & $16.0 \%$ \\
\hline & \multirow[t]{2}{*}{ multiple } & count & 0 & 0 & 1 & 1 \\
\hline & & $\%$ within raw material & $0.0 \%$ & $0.0 \%$ & $4.5 \%$ & $4.0 \%$ \\
\hline \multirow{2}{*}{\multicolumn{2}{|c|}{ Total }} & count & 2 & 1 & 22 & 25 \\
\hline & & $\%$ within raw material & $100.0 \%$ & $100.0 \%$ & $100.0 \%$ & $100.0 \%$ \\
\hline
\end{tabular}


Table 147. Esztergom blade assemblage (complete specimens) platform type frequency by raw material

\begin{tabular}{|c|c|c|c|c|c|c|}
\hline & \multicolumn{3}{|c|}{ Raw material } & \multirow{2}{*}{ Total } \\
\hline & & & regional & distant & transcarpathian & \\
\hline \multirow[t]{10}{*}{ Platform } & \multirow[t]{2}{*}{ plain } & count & 0 & 1 & 13 & 14 \\
\hline & & $\%$ within raw material & $0.0 \%$ & $100.0 \%$ & $59.1 \%$ & $56.0 \%$ \\
\hline & \multirow[t]{2}{*}{ dihedral } & count & 1 & 0 & 1 & 2 \\
\hline & & $\%$ within raw material & $50.0 \%$ & $0.0 \%$ & $4.5 \%$ & $8.0 \%$ \\
\hline & \multirow[t]{2}{*}{ faceted } & count & 0 & 0 & 5 & 5 \\
\hline & & $\%$ within raw material & $0.0 \%$ & $0.0 \%$ & $22.7 \%$ & $20.0 \%$ \\
\hline & \multirow[t]{2}{*}{ linear } & count & 1 & 0 & 2 & 3 \\
\hline & & $\%$ within raw material & $50.0 \%$ & $0.0 \%$ & $9.1 \%$ & $12.0 \%$ \\
\hline & \multirow[t]{2}{*}{ irregular } & count & 0 & 0 & 1 & 1 \\
\hline & & $\%$ within raw material & $0.0 \%$ & $0.0 \%$ & $4.5 \%$ & $4.0 \%$ \\
\hline \multirow{2}{*}{\multicolumn{2}{|c|}{ Total }} & count & 2 & 1 & 22 & 25 \\
\hline & & $\%$ within raw material & $100.0 \%$ & $100.0 \%$ & $100.0 \%$ & $100.0 \%$ \\
\hline
\end{tabular}

Table 148. Esztergom blade assemblage (complete specimens) impact point-overhang co-presence by raw material

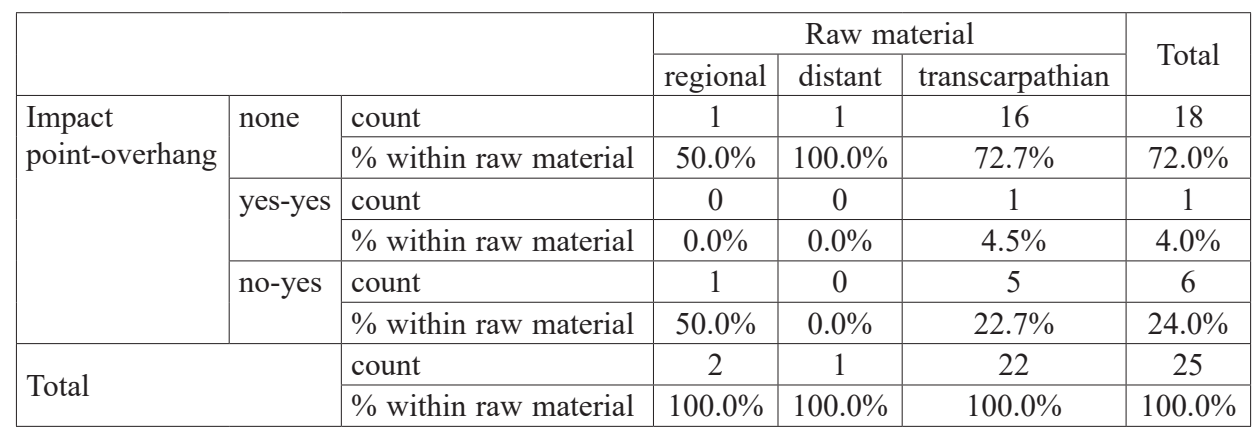


Table 149. Esztergom blade assemblage (complete specimens) length, width and thickness by raw material

\begin{tabular}{|c|c|c|c|c|}
\hline \multicolumn{2}{|l|}{ Raw material } & Length [mm] & Width [mm] & Thickness [mm] \\
\hline \multirow[t]{6}{*}{ Regional } & Minimum & 30.00 & 8.10 & 3.70 \\
\hline & Maximum & 37.60 & 14.50 & 5.10 \\
\hline & Median & 33.8000 & 11.3000 & 4.4000 \\
\hline & Mean & 33.8000 & 11.3000 & 4.4000 \\
\hline & $\mathrm{N}$ & 2 & 2 & 2 \\
\hline & Std. deviation & 5.37401 & 4.52548 & 0.98995 \\
\hline \multirow[t]{6}{*}{ Distant } & Minimum & 36.70 & 11.00 & 8.20 \\
\hline & Maximum & 36.70 & 11.00 & 8.20 \\
\hline & Median & 36.7000 & 11.0000 & 8.2000 \\
\hline & Mean & 36.7000 & 11.0000 & 8.2000 \\
\hline & $\mathrm{N}$ & 1 & 1 & 1 \\
\hline & Std. deviation & - & - & - \\
\hline \multirow[t]{6}{*}{ Transcarpathian } & Minimum & 21.20 & 6.60 & 2.50 \\
\hline & Maximum & 93.30 & 32.20 & 12.50 \\
\hline & Median & 46.9000 & 14.3000 & 5.1000 \\
\hline & Mean & 48.2045 & 15.7364 & 5.7545 \\
\hline & $\mathrm{N}$ & 22 & 22 & 22 \\
\hline & Std. deviation & 21.31783 & 7.14793 & 2.73996 \\
\hline \multirow[t]{6}{*}{ Total } & Minimum & 21.20 & 6.60 & 2.50 \\
\hline & Maximum & 93.30 & 32.20 & 12.50 \\
\hline & Median & 38.7000 & 13.4000 & 5.1000 \\
\hline & Mean & 46.5920 & 15.1920 & 5.7440 \\
\hline & $\mathrm{N}$ & 25 & 25 & 25 \\
\hline & Std. deviation & 20.46812 & 6.91562 & 2.64797 \\
\hline
\end{tabular}

Table 150. Esztergom blade assemblage (complete specimens) t-test to compare length, width and thickness by raw materials

\begin{tabular}{|c|c|c|c|c|c|c|c|}
\hline \multicolumn{8}{|c|}{ Independent Samples Test } \\
\hline & \multicolumn{2}{|c|}{$\begin{array}{l}\text { Levene's Test } \\
\text { for Equality } \\
\text { of Variances }\end{array}$} & \multicolumn{5}{|c|}{ t-test for Equality of Means } \\
\hline & $\mathrm{F}$ & Sig. & $\mathrm{t}$ & df & Sig. (2-tailed) & $\begin{array}{c}\text { Mean } \\
\text { difference }\end{array}$ & $\begin{array}{l}\text { Std. error } \\
\text { difference }\end{array}$ \\
\hline \multirow{2}{*}{$\begin{array}{l}\text { Length } \\
{[\mathrm{mm}]}\end{array}$} & 3.888 & 0.061 & -0.935 & 22 & 0.360 & -14.40455 & 15.40553 \\
\hline & & & -2.431 & 5.383 & 0.056 & -14.40455 & 5.92426 \\
\hline \multirow{2}{*}{$\begin{array}{l}\text { Width } \\
{[\mathrm{mm}]}\end{array}$} & 1.041 & 0.319 & -0.852 & 22 & 0.403 & -4.43636 & 5.20671 \\
\hline & & & -1.252 & 1.501 & 0.371 & -4.43636 & 3.54435 \\
\hline \multirow{2}{*}{$\begin{array}{l}\text { Thickness } \\
{[\mathrm{mm}]}\end{array}$} & 1.700 & 0.206 & -0.683 & 22 & 0.502 & -1.35455 & 1.98320 \\
\hline & & & -1.486 & 2.813 & 0.240 & -1.35455 & 0.91173 \\
\hline
\end{tabular}


Table 151. Esztergom tool assemblage product composition by raw materials

\begin{tabular}{|c|c|c|c|c|}
\hline & \multicolumn{2}{|c|}{ Raw material } & \multirow{2}{*}{ Total } \\
\hline & & regional & transcarpathian & \\
\hline \multirow[t]{2}{*}{ Flake } & count & 0 & 26 & 26 \\
\hline & $\%$ within raw material & $0.0 \%$ & $7.8 \%$ & $7.6 \%$ \\
\hline \multirow[t]{2}{*}{ Blade } & count & 9 & 309 & 318 \\
\hline & $\%$ within raw material & $100.0 \%$ & $92.2 \%$ & $92.4 \%$ \\
\hline \multirow{2}{*}{ Total } & count & 9 & 335 & 344 \\
\hline & $\%$ within raw material & $100.0 \%$ & $100.0 \%$ & $100.0 \%$ \\
\hline
\end{tabular}

Table 152. Esztergom knapped product frequency in tool assemblage by raw materials

\begin{tabular}{|c|c|c|c|c|c|c|c|}
\hline & \multicolumn{3}{|c|}{ Raw material } & \multirow{2}{*}{ Total } \\
\hline & & & & regional & distant & transcarpathian & \\
\hline \multirow{6}{*}{ Flake } & \multirow{4}{*}{ state } & \multirow{2}{*}{ blank } & count & 34 & & 163 & 197 \\
\hline & & & $\%$ within raw material & $100.0 \%$ & & $86.2 \%$ & $88.3 \%$ \\
\hline & & \multirow{2}{*}{ tool } & count & 0 & & 26 & 26 \\
\hline & & & $\%$ within raw material & $0.0 \%$ & & $13.8 \%$ & $11.7 \%$ \\
\hline & \multirow{2}{*}{\multicolumn{2}{|c|}{ total }} & count & 34 & & 189 & 223 \\
\hline & & & $\%$ within raw material & $100.0 \%$ & & $100.0 \%$ & $100.0 \%$ \\
\hline \multirow{6}{*}{ Blade } & \multirow{4}{*}{ state } & \multirow{2}{*}{ blank } & count & 10 & 1 & 215 & 226 \\
\hline & & & $\%$ within raw material & $52.6 \%$ & $100.0 \%$ & $41.0 \%$ & $41.5 \%$ \\
\hline & & \multirow{2}{*}{ tool } & count & 9 & 0 & 309 & 318 \\
\hline & & & $\%$ within raw material & $47.4 \%$ & $0.0 \%$ & $59.0 \%$ & $58.5 \%$ \\
\hline & \multirow{2}{*}{\multicolumn{2}{|c|}{ total }} & count & 19 & 1 & 524 & 544 \\
\hline & & & $\%$ within raw material & $100.0 \%$ & $100.0 \%$ & $100.0 \%$ & $100.0 \%$ \\
\hline \multirow{4}{*}{ Debris } & \multirow{2}{*}{ state } & \multirow{2}{*}{ blank } & count & 11 & & & \\
\hline & & & \% within raw material & $100.0 \%$ & & & \\
\hline & \multirow{2}{*}{\multicolumn{2}{|c|}{ total }} & count & 11 & & & \\
\hline & & & $\%$ within raw material & $100.0 \%$ & & & \\
\hline
\end{tabular}

Table 153. Esztergom blade tool assemblage length, width and thickness by raw materials

\begin{tabular}{|l|l|c|c|c|}
\hline Raw material & & Length [mm] & Width [mm] & Thickness [mm] \\
\hline \multirow{5}{*}{ Regional } & Minimum & 14.30 & 6.20 & 2.40 \\
\cline { 2 - 5 } & Maximum & 56.20 & 27.60 & 11.60 \\
\cline { 2 - 5 } & Mean & 30.5444 & 13.9667 & 4.9333 \\
\cline { 2 - 5 } & Median & 29.8000 & 13.1000 & 3.6000 \\
\cline { 2 - 5 } & N & 9 & 9 & 9 \\
\cline { 2 - 5 } & Std. deviation & 14.73067 & 5.80668 & 2.77669 \\
\hline \multirow{5}{*}{ Transcarpathian } & Minimum & 9.10 & 5.80 & 1.60 \\
\cline { 2 - 5 } & Maximum & 103.80 & 32.20 & 14.80 \\
\cline { 2 - 5 } & Mean & 34.2045 & 15.0278 & 4.9667 \\
\cline { 2 - 5 } & Median & 32.2000 & 14.2000 & 4.5000 \\
\cline { 2 - 5 } & N & 309 & 309 & 309 \\
\cline { 2 - 5 } & Std. deviation & 15.05228 & 4.26573 & 1.99083 \\
\hline & Minimum & 9.10 & 5.80 & 14.80 \\
\cline { 2 - 5 } & Maximum & 103.80 & 32.20 & 4.9657 \\
\cline { 2 - 5 } & Mean & 34.1009 & 14.9978 & 318 \\
\cline { 2 - 5 } & Median & 32.1000 & 14.2000 & 2.01134 \\
\cline { 2 - 5 } & N & 318 & 4.30834 & \\
\cline { 2 - 5 } & Std. deviation & 15.03277 & & \\
\hline
\end{tabular}


Table 154. Esztergom flint blade blank and tool length, width and thickness mean t-test comparison

\begin{tabular}{|c|c|c|c|c|c|c|c|c|}
\hline \multicolumn{9}{|c|}{ Independent Samples Test } \\
\hline \multirow{2}{*}{\multicolumn{2}{|c|}{ Raw material }} & \multicolumn{2}{|c|}{$\begin{array}{l}\text { Levene's Test } \\
\text { for Equality } \\
\text { of Variances }\end{array}$} & \multicolumn{5}{|c|}{ t-test for Equality of Means } \\
\hline & & $\mathrm{F}$ & Sig. & $\mathrm{t}$ & df & $\begin{array}{c}\text { Sig. } \\
\text { (2-tailed) }\end{array}$ & $\begin{array}{c}\text { Mean } \\
\text { difference }\end{array}$ & $\begin{array}{l}\text { Std. error } \\
\text { difference }\end{array}$ \\
\hline \multirow[t]{6}{*}{ Regional } & \multirow{2}{*}{$\begin{array}{l}\text { length } \\
{[\mathrm{mm}]}\end{array}$} & 1.110 & 0.320 & 0.297 & 9 & 0.773 & 3.25556 & 10.94685 \\
\hline & & & & 0.524 & 5.285 & 0.621 & 3.25556 & 6.20889 \\
\hline & \multirow{2}{*}{$\begin{array}{l}\text { width } \\
{[\mathrm{mm}]}\end{array}$} & 0.014 & 0.910 & -0.601 & 9 & 0.563 & -2.66667 & 4.43918 \\
\hline & & & & -0.713 & 1.835 & 0.556 & -2.66667 & 3.73984 \\
\hline & \multirow{2}{*}{\begin{tabular}{|l|} 
thickness \\
{$[\mathrm{mm}]$}
\end{tabular}} & 0.750 & 0.409 & -0.259 & 9 & 0.802 & -0.53333 & 2.06269 \\
\hline & & & & -0.460 & 5.465 & 0.664 & -0.53333 & 1.16046 \\
\hline \multirow[t]{6}{*}{ Transcarpathian } & \multirow{2}{*}{$\begin{array}{l}\text { length } \\
{[\mathrm{mm}]}\end{array}$} & 9.774 & 0.002 & 4.086 & 329 & 0.000 & 14.00001 & 3.42639 \\
\hline & & & & 3.027 & 22.515 & 0.006 & 14.00001 & 4.62494 \\
\hline & \multirow{2}{*}{$\begin{array}{l}\text { width } \\
\text { [mm] }\end{array}$} & 18.731 & 0.000 & 0.713 & 329 & 0.477 & 0.70853 & 0.99410 \\
\hline & & & & 0.459 & 22.078 & 0.651 & 0.70853 & 1.54314 \\
\hline & \multirow{2}{*}{$\begin{array}{l}\text { thickness } \\
{[\mathrm{mm}]}\end{array}$} & 5.762 & 0.017 & 1.744 & 329 & 0.082 & 0.78788 & 0.45166 \\
\hline & & & & 1.324 & 22.606 & 0.199 & 0.78788 & 0.59504 \\
\hline
\end{tabular}

Table 155. Esztergom blade tool length, width and thickness mean comparison by raw materials with t-test and the Tukey post hoc

\begin{tabular}{|c|c|c|c|c|c|c|c|}
\hline \multicolumn{8}{|c|}{ Independent Samples Test } \\
\hline & \multicolumn{2}{|c|}{$\begin{array}{l}\text { Levene's Test } \\
\text { for Equality } \\
\text { of Variances }\end{array}$} & \multicolumn{5}{|c|}{ t-test for Equality of Means } \\
\hline & $\mathrm{F}$ & Sig. & $\mathrm{t}$ & df & Sig. (2-tailed) & $\begin{array}{c}\text { Mean } \\
\text { difference }\end{array}$ & $\begin{array}{l}\text { Std. error } \\
\text { difference }\end{array}$ \\
\hline \multirow{2}{*}{$\begin{array}{l}\text { Length } \\
{[\mathrm{mm}]}\end{array}$} & 0.117 & 0.733 & -0.719 & 316 & 0.472 & -3.66009 & 5.08725 \\
\hline & & & -0.734 & 8.494 & 0.483 & -3.66009 & 4.98433 \\
\hline \multirow{2}{*}{$\begin{array}{l}\text { Width } \\
{[\mathrm{mm}]}\end{array}$} & 0.184 & 0.668 & -0.728 & 316 & 0.467 & -1.06117 & 1.45796 \\
\hline & & & -0.544 & 8.253 & 0.601 & -1.06117 & 1.95071 \\
\hline \multirow{2}{*}{$\begin{array}{l}\text { Thickness } \\
{[\mathrm{mm}]}\end{array}$} & 0.935 & 0.334 & -0.049 & 316 & 0.961 & -0.03333 & 0.68121 \\
\hline & & & -0.036 & 8.241 & 0.972 & -0.03333 & 0.93247 \\
\hline
\end{tabular}


Lithic analysis of the Middle and Late Upper Palaeolithic in Hungary

Table 156. Esztergom blade tool types by raw material

\begin{tabular}{|c|c|c|c|c|c|}
\hline & \multicolumn{2}{|c|}{ Raw material } & \multirow{2}{*}{ Total } \\
\hline & & & regional & transcarpathian & \\
\hline \multirow[t]{16}{*}{ Tooltypes } & \multirow[t]{2}{*}{ endscraper } & count & 0 & 4 & 4 \\
\hline & & $\%$ within raw material & $0.0 \%$ & $1.3 \%$ & $1.3 \%$ \\
\hline & \multirow[t]{2}{*}{ burin } & count & 0 & 25 & 25 \\
\hline & & $\%$ within raw material & $0.0 \%$ & $8.1 \%$ & $7.9 \%$ \\
\hline & \multirow[t]{2}{*}{ retouched } & count & 2 & 48 & 50 \\
\hline & & $\%$ within raw material & $22.2 \%$ & $15.5 \%$ & $15.7 \%$ \\
\hline & \multirow[t]{2}{*}{ borer } & count & 0 & 2 & 2 \\
\hline & & $\%$ within raw material & $0.0 \%$ & $0.6 \%$ & $0.6 \%$ \\
\hline & \multirow[t]{2}{*}{ truncation } & count & 0 & 8 & 8 \\
\hline & & $\%$ within raw material & $0.0 \%$ & $2.6 \%$ & $2.5 \%$ \\
\hline & \multirow{2}{*}{$\begin{array}{l}\text { notched- } \\
\text { denticulated }\end{array}$} & count & 0 & 1 & 1 \\
\hline & & $\%$ within raw material & $0.0 \%$ & $0.3 \%$ & $0.3 \%$ \\
\hline & \multirow[t]{2}{*}{ composite } & count & 0 & 1 & 1 \\
\hline & & $\%$ within raw material & $0.0 \%$ & $0.3 \%$ & $0.3 \%$ \\
\hline & \multirow[t]{2}{*}{ armature } & count & 7 & 220 & 227 \\
\hline & & $\%$ within raw material & $77.8 \%$ & $71.2 \%$ & $71.4 \%$ \\
\hline \multirow{2}{*}{\multicolumn{2}{|c|}{ Total }} & count & 9 & 309 & 318 \\
\hline & & $\%$ within raw material & $100.0 \%$ & $100.0 \%$ & $100.0 \%$ \\
\hline
\end{tabular}

Table 157. Esztergom armature types by raw material

\begin{tabular}{|c|c|c|c|c|c|}
\hline & \multicolumn{2}{|c|}{ Raw material } & \multirow{2}{*}{ Total } \\
\hline & & & regional & transcarpathian & \\
\hline \multirow[t]{10}{*}{ Armatures } & \multirow[t]{2}{*}{ backed } & count & 6 & 129 & 135 \\
\hline & & $\%$ within raw material & $85.7 \%$ & $58.6 \%$ & $59.5 \%$ \\
\hline & \multirow{2}{*}{$\begin{array}{l}\text { backed- } \\
\text { truncated }\end{array}$} & count & 1 & 50 & 51 \\
\hline & & $\%$ within raw material & $14.3 \%$ & $22.7 \%$ & $22.5 \%$ \\
\hline & \multirow[t]{2}{*}{ trapeze } & count & 0 & 3 & 3 \\
\hline & & $\%$ within raw material & $0.0 \%$ & $1.4 \%$ & $1.3 \%$ \\
\hline & \multirow{2}{*}{$\begin{array}{l}\text { trapeze- } \\
\text { rectangle }\end{array}$} & count & 0 & 5 & 5 \\
\hline & & $\%$ within raw material & $0.0 \%$ & $2.3 \%$ & $2.2 \%$ \\
\hline & \multirow[t]{2}{*}{ points } & count & 0 & 33 & 33 \\
\hline & & $\%$ within raw material & $0.0 \%$ & $15.0 \%$ & $14.5 \%$ \\
\hline \multirow{2}{*}{\multicolumn{2}{|c|}{ Total }} & count & 7 & 220 & 227 \\
\hline & & $\%$ within raw material & $100.0 \%$ & $100.0 \%$ & $100.0 \%$ \\
\hline
\end{tabular}


Table 158. Esztergom point types by raw material

\begin{tabular}{|c|c|c|c|c|}
\hline & Raw material & \multirow{2}{*}{ Total } \\
\hline & & & transcarpathian & \\
\hline \multirow[t]{10}{*}{ Points } & \multirow[t]{2}{*}{ retouched } & count & 6 & 6 \\
\hline & & $\%$ within raw material & $18.2 \%$ & $18.2 \%$ \\
\hline & \multirow{2}{*}{$\begin{array}{l}\text { gravette/ } \\
\text { microgravette }\end{array}$} & count & 1 & 1 \\
\hline & & $\%$ within raw material & $3.0 \%$ & $3.0 \%$ \\
\hline & \multirow[t]{2}{*}{ backed } & count & 5 & 5 \\
\hline & & $\%$ within raw material & $15.2 \%$ & $15.2 \%$ \\
\hline & \multirow[t]{2}{*}{ arched backed } & count & 2 & 2 \\
\hline & & $\%$ within raw material & $6.1 \%$ & $6.1 \%$ \\
\hline & \multirow[t]{2}{*}{ curved backed } & count & 19 & 19 \\
\hline & & $\%$ within raw material & $57.6 \%$ & $57.6 \%$ \\
\hline \multirow{2}{*}{\multicolumn{2}{|c|}{ Total }} & count & 33 & 33 \\
\hline & & $\%$ within raw material & $100.0 \%$ & $100.0 \%$ \\
\hline
\end{tabular}

Table 159. Esztergom flake assemblage (complete specimens) dorsal scar pattern frequency by raw material

\begin{tabular}{|c|c|c|c|c|c|}
\hline & \multicolumn{2}{|c|}{ Raw material } & \multirow{2}{*}{ Total } \\
\hline & & & regional & transcarpathian & \\
\hline \multirow[t]{10}{*}{ Scars } & \multirow[t]{2}{*}{ unidirectional } & count & 4 & 15 & 19 \\
\hline & & $\%$ within raw material & $40.0 \%$ & $40.5 \%$ & $40.4 \%$ \\
\hline & \multirow[t]{2}{*}{ opposite } & count & 1 & 3 & 4 \\
\hline & & $\%$ within raw material & $10.0 \%$ & $8.1 \%$ & $8.5 \%$ \\
\hline & \multirow[t]{2}{*}{ perpendicular } & count & 5 & 13 & 18 \\
\hline & & $\%$ within raw material & $50.0 \%$ & $35.1 \%$ & $38.3 \%$ \\
\hline & \multirow[t]{2}{*}{ multiple } & count & 0 & 5 & 5 \\
\hline & & $\%$ within raw material & $0.0 \%$ & $13.5 \%$ & $10.6 \%$ \\
\hline & \multirow[t]{2}{*}{ no scar } & count & 0 & 1 & 1 \\
\hline & & $\%$ within raw material & $0.0 \%$ & $2.7 \%$ & $2.1 \%$ \\
\hline \multirow{2}{*}{\multicolumn{2}{|c|}{ Total }} & count & 10 & 37 & 47 \\
\hline & & $\%$ within raw material & $100.0 \%$ & $100.0 \%$ & $100.0 \%$ \\
\hline
\end{tabular}


Table 160. Esztergom flake assemblage (complete specimens) platform type frequency by raw material

\begin{tabular}{|c|c|c|c|c|c|}
\hline & \multicolumn{2}{|c|}{ Raw material } & \multirow{2}{*}{ Total } \\
\hline & & & regional & transcarpathian & \\
\hline \multirow[t]{14}{*}{ Platform } & \multirow[t]{2}{*}{ plain } & count & 5 & 22 & 27 \\
\hline & & $\%$ within raw material & $50.0 \%$ & $59.5 \%$ & $57.4 \%$ \\
\hline & \multirow[t]{2}{*}{ dihedral } & count & 1 & 1 & 2 \\
\hline & & $\%$ within raw material & $10.0 \%$ & $2.7 \%$ & $4.3 \%$ \\
\hline & \multirow[t]{2}{*}{ faceted } & count & 2 & 7 & 9 \\
\hline & & $\%$ within raw material & $20.0 \%$ & $18.9 \%$ & $19.1 \%$ \\
\hline & \multirow[t]{2}{*}{ cortical } & count & 1 & 1 & 2 \\
\hline & & $\%$ within raw material & $10.0 \%$ & $2.7 \%$ & $4.3 \%$ \\
\hline & \multirow[t]{2}{*}{ linear } & count & 0 & 4 & 4 \\
\hline & & $\%$ within raw material & $0.0 \%$ & $10.8 \%$ & $8.5 \%$ \\
\hline & \multirow[t]{2}{*}{ punctiform } & count & 0 & 2 & 2 \\
\hline & & $\%$ within raw material & $0.0 \%$ & $5.4 \%$ & $4.3 \%$ \\
\hline & \multirow[t]{2}{*}{ irregular } & count & 1 & 0 & 1 \\
\hline & & $\%$ within raw material & $10.0 \%$ & $0.0 \%$ & $2.1 \%$ \\
\hline \multirow{2}{*}{\multicolumn{2}{|c|}{ Total }} & count & 10 & 37 & 47 \\
\hline & & $\%$ within raw material & $100.0 \%$ & $100.0 \%$ & $100.0 \%$ \\
\hline
\end{tabular}

Table 161. Esztergom flake assemblage (complete specimens) impact point-overhang frequency by raw material

\begin{tabular}{|c|c|c|c|c|c|}
\hline & \multicolumn{2}{|c|}{ Raw material } & \multirow{2}{*}{ Total } \\
\hline & & & regional & transcarpathian & \\
\hline \multirow{8}{*}{$\begin{array}{l}\text { Impact } \\
\text { point-overhang }\end{array}$} & \multirow[t]{2}{*}{ none } & count & 1 & 17 & 18 \\
\hline & & $\%$ within raw material & $10.0 \%$ & $45.9 \%$ & $38.3 \%$ \\
\hline & \multirow[t]{2}{*}{ yes-no } & count & 0 & 4 & 4 \\
\hline & & $\%$ within raw material & $0.0 \%$ & $10.8 \%$ & $8.5 \%$ \\
\hline & \multirow[t]{2}{*}{ yes-yes } & count & 2 & 7 & 9 \\
\hline & & $\%$ within raw material & $20.0 \%$ & $18.9 \%$ & $19.1 \%$ \\
\hline & \multirow[t]{2}{*}{ no-yes } & count & 7 & 9 & 16 \\
\hline & & $\%$ within raw material & $70.0 \%$ & $24.3 \%$ & $34.0 \%$ \\
\hline \multirow{2}{*}{\multicolumn{2}{|c|}{ Total }} & count & 10 & 37 & 47 \\
\hline & & $\%$ within raw material & $100.0 \%$ & $100.0 \%$ & $100.0 \%$ \\
\hline
\end{tabular}


Table 162. Esztergom flake assemblage (complete specimens) length, width and thickness by raw material

\begin{tabular}{|l|l|c|c|c|}
\hline Raw material & & Length [mm] & Width [mm] & Thickness [mm] \\
\hline \multirow{5}{*}{ Regional } & Minimum & 22.10 & 22.10 & 3.70 \\
\cline { 2 - 5 } & Maximum & 61.50 & 82.60 & 19.80 \\
\cline { 2 - 5 } & Mean & 41.4100 & 40.1800 & 11.9800 \\
\cline { 2 - 5 } & Median & 42.4000 & 35.2500 & 12.6000 \\
\cline { 2 - 5 } & N & 10 & 10 & 10 \\
\cline { 2 - 5 } & Std. deviation & 14.42625 & 20.56085 & 4.39060 \\
\hline \multirow{5}{*}{ Transcarpathian } & Minimum & 13.40 & 9.90 & 2.00 \\
\cline { 2 - 5 } & Maximum & 64.10 & 45.90 & 11.60 \\
\cline { 2 - 5 } & Mean & 30.1919 & 22.9027 & 5.7243 \\
\cline { 2 - 5 } & Median & 30.4000 & 22.5000 & 4.8000 \\
\cline { 2 - 5 } & N & 37 & 37 & 37 \\
\cline { 2 - 5 } & Std. deviation & 13.79872 & 9.00313 & 2.84316 \\
\hline & Minimum & 13.40 & 9.90 & 19.80 \\
\cline { 2 - 5 } & Maximum & 64.10 & 82.60 & 6.0553 \\
\cline { 2 - 5 } & Mean & 32.5787 & 26.5787 & 47 \\
\cline { 2 - 5 } & Median & 32.2000 & 4.7000 & 4.09819 \\
\cline { 2 - 5 } & $\mathrm{N}$ & 47.53505 & 14.04395 & \\
\cline { 2 - 5 } & Std. deviation & & & \\
\hline
\end{tabular}

Table 163. Esztergom flake assemblage (complete specimens) t-test to compare length, width and thickness by raw materials

\begin{tabular}{|l|c|c|c|c|c|c|c|}
\hline \multicolumn{1}{|c|}{} & $\begin{array}{c}\text { Levene's Test } \\
\text { for Equality } \\
\text { of Variances }\end{array}$ & \multicolumn{7}{|c|}{ Independent Samples Test } \\
\cline { 2 - 8 } & $\mathrm{F}$ & Sig. & $\mathrm{t}$ & $\mathrm{df}$ & $\begin{array}{c}\text { Sig. } \\
\text { (2-tailed) }\end{array}$ & $\begin{array}{c}\text { Mean } \\
\text { difference }\end{array}$ & $\begin{array}{c}\text { Std. error } \\
\text { difference }\end{array}$ \\
\hline \multirow{2}{*}{$\begin{array}{l}\text { Length } \\
{[\mathrm{mm}]}\end{array}$} & 0.042 & 0.838 & 2.260 & 45 & 0.029 & 11.21811 & 4.96352 \\
\cline { 2 - 8 } $\begin{array}{l}\text { Width } \\
{[\mathrm{mm}]}\end{array}$ & 8.136 & 0.007 & 3.966 & 45 & 0.000 & 17.21811 & 5.09487 \\
\cline { 2 - 8 } & & & 2.591 & 9.950 & 0.027 & 17.27730 & 6.35628 \\
\hline $\begin{array}{l}\text { Thickness } \\
{[\mathrm{mm}]}\end{array}$ & 1.351 & 0.251 & 5.463 & 45 & 0.000 & 6.25568 & 1.14508 \\
\cline { 2 - 8 } & & & 4.270 & 11.120 & 0.001 & 6.25568 & 1.46499 \\
\hline
\end{tabular}

Table 164. Esztergom flake tool assemblage length, width and thickness by raw materials

\begin{tabular}{|l|l|c|c|c|}
\hline Raw material & & Length [mm] & Width [mm] & Thickness [mm] \\
\hline \multirow{5}{*}{ Transcarpathian } & Minimum & 14.90 & 11.30 & 2.60 \\
\cline { 2 - 5 } & Maximum & 59.30 & 56.40 & 17.90 \\
\cline { 2 - 5 } & Median & 30.2500 & 23.0000 & 5.6500 \\
\cline { 2 - 5 } & Mean & 34.6731 & 25.4808 & 6.2269 \\
\cline { 2 - 5 } & N & 26 & 26 & 26 \\
\cline { 2 - 5 } & Std. deviation & 14.90921 & 12.06758 & 3.54644 \\
\hline \multirow{5}{*}{ Total } & Minimum & 14.90 & 11.30 & 2.60 \\
\cline { 2 - 5 } & Maximum & 59.30 & 56.40 & 17.90 \\
\cline { 2 - 5 } & Median & 30.2500 & 23.0000 & 5.6500 \\
\cline { 2 - 5 } & Mean & 34.6731 & 25.4808 & 6.2269 \\
\cline { 2 - 5 } & N & 26 & 26 & 26 \\
\cline { 2 - 5 } & Std. deviation & 14.90921 & 12.06758 & 3.54644 \\
\hline
\end{tabular}


Lithic analysis of the Middle and Late Upper Palaeolithic in Hungary

Table 165. Esztergom flake blank and tool length, width and thickness mean t-test comparison (only transcarpathian raw material)

\begin{tabular}{|l|c|c|c|c|c|c|c|}
\hline \multicolumn{1}{|c|}{} & $\begin{array}{c}\text { Levene's Test } \\
\text { for Equality } \\
\text { of Variances }\end{array}$ & \multicolumn{6}{|c|}{ Independent Samples Test } \\
\cline { 2 - 8 } & $\mathrm{F}$ & Sig. & $\mathrm{t}$ & $\mathrm{df}$ & $\begin{array}{c}\text { Sig. } \\
(2 \text {-tailed })\end{array}$ & $\begin{array}{c}\text { Mean } \\
\text { difference }\end{array}$ & $\begin{array}{c}\text { Std. error } \\
\text { difference }\end{array}$ \\
\hline $\begin{array}{l}\text { Length } \\
{[\mathrm{mm}]}\end{array}$ & 0.436 & 0.511 & -0.584 & 71 & 0.561 & -2.09435 & 3.58503 \\
\hline $\begin{array}{l}\text { Width } \\
{[\mathrm{mm}]}\end{array}$ & 0.105 & 0.747 & 0.336 & 71 & 0.738 & 1.09795 & 3.61171 \\
\hline $\begin{array}{l}\text { Thickness } \\
{[\mathrm{mm}]}\end{array}$ & 1.232 & 0.271 & 0.866 & 71 & 0.389 & 0.82840 & 3.13009 \\
\cline { 2 - 8 } & & & 0.903 & 58.290 & 0.370 & 0.82840 & 0.95634 \\
\hline
\end{tabular}

Table 166. Esztergom flake tool types (only transcarpathian raw material)

\begin{tabular}{|c|c|c|c|c|}
\hline & \multirow{2}{*}{$\begin{array}{c}\text { Raw material } \\
\text { transcarpathian }\end{array}$} & \multirow{2}{*}{ Total } \\
\hline & & & & \\
\hline \multirow[t]{10}{*}{ Tooltypes } & \multirow[t]{2}{*}{ burin } & count & 5 & 5 \\
\hline & & $\%$ within raw material & $19.2 \%$ & $19.2 \%$ \\
\hline & \multirow[t]{2}{*}{ retouched } & count & 9 & 9 \\
\hline & & $\%$ within raw material & $34.6 \%$ & $34.6 \%$ \\
\hline & \multirow[t]{2}{*}{ borer } & count & 1 & 1 \\
\hline & & $\%$ within raw material & $3.8 \%$ & $3.8 \%$ \\
\hline & \multirow[t]{2}{*}{ armature } & count & 8 & 8 \\
\hline & & $\%$ within raw material & $30.8 \%$ & $30.8 \%$ \\
\hline & \multirow{2}{*}{$\begin{array}{l}\text { notched- } \\
\text { denticulated }\end{array}$} & count & 3 & 3 \\
\hline & & $\%$ within raw material & $11.5 \%$ & $11.5 \%$ \\
\hline \multirow{2}{*}{\multicolumn{2}{|c|}{ Total }} & count & 26 & 26 \\
\hline & & $\%$ within raw material & $100.0 \%$ & $100.0 \%$ \\
\hline
\end{tabular}

Table 167. Esztergom flake armature types (only transcarpathian raw material)

\begin{tabular}{|c|c|c|c|c|}
\hline & Raw material & \multirow{2}{*}{ Total } \\
\hline & & & transcarpathian & \\
\hline \multirow[t]{4}{*}{ Armature } & \multirow[t]{2}{*}{ backed } & count & 7 & 7 \\
\hline & & $\%$ within raw material & $87.5 \%$ & $87.5 \%$ \\
\hline & \multirow[t]{2}{*}{ rectangle } & count & 1 & 1 \\
\hline & & \% within raw material & $12.5 \%$ & $12.5 \%$ \\
\hline \multirow{2}{*}{\multicolumn{2}{|c|}{ Total }} & count & 8 & 8 \\
\hline & & \% within raw material & $100.0 \%$ & $100.0 \%$ \\
\hline
\end{tabular}




\section{The site}

The site is located in the south eastern part of Velencei Mountains, near Lake Velencei, at an elevation of about $180 \mathrm{~m}$ a.s.l. at the margin of an abandoned stone quarry (Dobosi et al. 1988). About 40 square meter area was recovered in 1985. By the time of the excavation the loess cover was already truncated by mining activity for sand.

The archaeological level was embedded in sandy slope loess. The first finds appeared $30 \mathrm{~cm}$ under the surface and the lowest finds lay at the depth of $40 \mathrm{~cm}$. No regularity was observed in the spatial distribution of the finds. Bones and knapped stones lay randomly. Two burnt spots, interpreted as hearths, were found in block E and in block H, respectively. These had vague outlines and their area contained poorly preserved charcoal grains. Animal bones in the area of the hearths bore signs of burning. Near the burnt spot in block E, a plastered surface of palm size was recovered.

The first chronological consideration of the site was based upon the Upper Pleistocene stratigraphy of Hungary (PÉCSI 1985). According to the excavators the human occupation lay under an embryonic soil called $\mathrm{h}_{2}$ and above the so called Mende Upper soil complex. The embryonic soil $\mathrm{h}_{2}$ was dated elsewhere to 20.5-21.7 ka BP and the Mende Upper soil complex to 32-27 ka BP (PÉCSI 1985). Based on this geological consideration, the human occupation at Nadap was placed to between 32 and $20 \mathrm{k}$ years BP. In contrast, the biostratigraphy of the site claimed that the hunted animal remains belonged to the Pilisszántó-Bajót fauna stage 18-12 ka BP (VöRös 2000). Radiocarbon dating of a horse phalange without stratigraphic precision of block D (Dobosi et al. 1988; A. Verpoorte personal communication 2009) yielded a date $13050 \pm 70$ BP (GrA-16563) (VERPOORTE 2004), which eventually corresponds with the biostratigraphy. Supporting what Verpoorte (2004) noticed, the detailed typological analysis of the assemblage pointed out a great similarity with the Late Epigravettian site of Esztergom (Lengyel 2016). Thus the fauna, radiocarbon dating and the lithic tool composition seem confirming the revised age of the assemblage.

The archaeological collection consists of animal bones and knapped lithics. This fauna is highly dominated by horse remains $(\mathrm{MNI}=35)$ and a few specimens of asinus, rein deer and bison.

\section{Raw materials}

The assemblage is dominated by cretaceous flints originating from the glacier outcrops (Tab. 168). Among the flints there are a few items that are similar to Jurassic flints. The transcarpathian material yielded 505.3 item per kilogram.

The second largest group of materials is the radiolarite of regional origin, from Gerecse Mountains (Doвosi et al. 1988), however these radiolarite items are also similar to those outcropping in the Pieniny Klippen Belt of the White Carpathians. The regional material yielded 400.4 per kilogram. 


\section{Blade tool production}

Both types of raw materials yielded products from a full sequence of debitage. The only element missing in transcarpathian material is the crest blade. Overall, blades dominate the industry and only blade cores were found in the assemblage (Tab. 169).

Although unidirectional scars are the most abundant on the blades (Tab. 170), they make up only less than $50 \%$ of the blade assemblage, which is in accordance with that out of the nine blade cores only two have single striking platform (Tab. 171). Blade platforms are plain (Tab. 172) without impact point and overhangs (Tab. 173) as a result of soft hammer percussion.

The mean length of the blades are very similar (Tab. 174) and the t-test found no differences between regional and transcarpathian blades mean size values (Tab. 175).

Blades rule the tool kit (Tab. 176) and out of the total blade assemblages $11.1 \%$ of the specimens are tools (Tab. 177).

Blade tools of both raw materials have similar sizes (Tab. 178). Regional blade tools are shorter and narrower than the blank blades (Tab. 179). Regarding transcarpathian items, the length and the width of the tools is smaller, and only the thicknesses are similar.

The size of the tools by raw materials is also very similar, the t-test found no difference (Tab. 180).

Most of the blade tools are armature (Tab. 181). Transcarpathian material yielded all the types of blade tools while the radiolarite was more used for armature. Among the armatures backed bladelets are common (Tab. 182). Among points, only two have straight back and the other three are curved or ached (Tab. 183).

\section{Flake tool production}

The flakes often were detached with unidirectional flaking (Tab. 184), their platforms are plain (Tab. 185), and several flakes have impact points of hard hammer percussion (Tab. 186).

The flakes are often short (Tab. 187) and their mean length, width and thickness do not differ by raw materials (Tab. 188).

Four flakes were retouched into tools (Tab. 176). The flake tools have similar mean lengths, widths and thicknesses compared to the blanks (Tab. 189). Comparing them with a statistical test gave no result due to low number of sample.

Flake tools were more frequently made of transcarpathian material, which are an end-scraper, a burin, an edge retouched tool and a truncated item (Tab. 190). 
Table 168. Nadap lithic raw material composition by weight in grams

\begin{tabular}{|l|c|c|c|c|c|}
\hline Raw material & Blade & Flake & Core & Total & $\%$ \\
\hline Regional & 391 & 113 & 63 & 567 & 24.98 \\
\hline Within regional & 68.95944 & 19.92945 & 11.11111 & 100 & \\
\hline Transcarpathian & 915 & 469 & 318 & 1702 & 75.02 \\
\hline Within transcarpathian & 53.76028 & 27.55582 & 18.6839 & 100 & \\
\hline Total & 1306 & 582 & 381 & 2269 & \\
\hline$\%$ & 57.5584 & 25.65007 & 16.79154 & 100 & \\
\hline
\end{tabular}

Table 169. Nadap lithic assemblage composition by raw material types and technological categories

\begin{tabular}{|c|c|c|c|c|c|}
\hline & \multicolumn{2}{|c|}{ Raw material } & \multirow{2}{*}{ Total } \\
\hline & & & regional & transcarpathian & \\
\hline \multirow[t]{14}{*}{ Class } & \multirow[t]{2}{*}{ flake } & count & 30 & 82 & 112 \\
\hline & & $\%$ within raw material & $13.2 \%$ & $9.5 \%$ & $10.3 \%$ \\
\hline & \multirow[t]{2}{*}{ blade } & count & 148 & 375 & 523 \\
\hline & & $\%$ within raw material & $65.2 \%$ & $43.6 \%$ & $48.1 \%$ \\
\hline & \multirow[t]{2}{*}{ debris } & count & 30 & 337 & 367 \\
\hline & & $\%$ within raw material & $13.2 \%$ & $39.2 \%$ & $33.8 \%$ \\
\hline & \multirow{2}{*}{$\begin{array}{l}\text { rejuvenating } \\
\text { flake }\end{array}$} & count & 9 & 29 & 38 \\
\hline & & $\%$ within raw material & $4.0 \%$ & $3.4 \%$ & $3.5 \%$ \\
\hline & \multirow[t]{2}{*}{ crest } & count & 1 & 0 & 1 \\
\hline & & $\%$ within raw material & $0.4 \%$ & $0.0 \%$ & $0.1 \%$ \\
\hline & \multirow[t]{2}{*}{ neo-crest } & count & 7 & 30 & 37 \\
\hline & & $\%$ within raw material & $3.1 \%$ & $3.5 \%$ & $3.4 \%$ \\
\hline & \multirow[t]{2}{*}{ blade core } & count & 2 & 7 & 9 \\
\hline & & $\%$ within raw material & $0.9 \%$ & $0.8 \%$ & $0.8 \%$ \\
\hline \multirow{2}{*}{\multicolumn{2}{|c|}{ Total }} & count & 227 & 860 & 1087 \\
\hline & & $\%$ within raw material & $100.0 \%$ & $100.0 \%$ & $100.0 \%$ \\
\hline
\end{tabular}

Table 170. Nadap blade assemblage (complete specimens) dorsal scar pattern frequency by raw material

\begin{tabular}{|c|c|c|c|c|c|}
\hline & \multicolumn{2}{|c|}{ Raw material } & \multirow{2}{*}{ Total } \\
\hline & & & regional & transcarpathian & \\
\hline \multirow[t]{8}{*}{ Scars } & \multirow[t]{2}{*}{ unidirectional } & count & 13 & 30 & 43 \\
\hline & & $\%$ within raw material & $52.0 \%$ & $42.3 \%$ & $44.8 \%$ \\
\hline & \multirow[t]{2}{*}{ opposite } & count & 10 & 26 & 36 \\
\hline & & $\%$ within raw material & $40.0 \%$ & $36.6 \%$ & $37.5 \%$ \\
\hline & \multirow[t]{2}{*}{ perpendicular } & count & 2 & 11 & 13 \\
\hline & & $\%$ within raw material & $8.0 \%$ & $15.5 \%$ & $13.5 \%$ \\
\hline & \multirow[t]{2}{*}{ multiple } & count & 0 & 4 & 4 \\
\hline & & $\%$ within raw material & $0.0 \%$ & $5.6 \%$ & $4.2 \%$ \\
\hline \multirow{2}{*}{\multicolumn{2}{|c|}{ Total }} & count & 25 & 71 & 96 \\
\hline & & $\%$ within raw material & $100.0 \%$ & $100.0 \%$ & $100.0 \%$ \\
\hline
\end{tabular}


Table 171. Nadap blade core types by raw material

\begin{tabular}{|c|c|c|c|c|c|c|}
\hline & \multicolumn{3}{|c|}{ Raw material } & \multirow{2}{*}{ Total } \\
\hline & & & regional & distant & transcarpathian & \\
\hline \multirow[t]{6}{*}{ Types } & \multirow[t]{2}{*}{ unidirectional } & count & 0 & 0 & 2 & 2 \\
\hline & & $\%$ within raw material & $0.0 \%$ & $0.0 \%$ & $28.6 \%$ & $22.2 \%$ \\
\hline & \multirow[t]{2}{*}{ bidirectional } & count & 1 & 0 & 1 & 2 \\
\hline & & $\%$ within raw material & $100.0 \%$ & $0.0 \%$ & $14.3 \%$ & $22.2 \%$ \\
\hline & \multirow[t]{2}{*}{ alternate } & count & 0 & 1 & 4 & 5 \\
\hline & & $\%$ within raw material & $0.0 \%$ & $100.0 \%$ & $57.1 \%$ & $55.6 \%$ \\
\hline \multirow{2}{*}{\multicolumn{2}{|c|}{ Total }} & count & 1 & 1 & 7 & 9 \\
\hline & & $\%$ within raw material & $100.0 \%$ & $100.0 \%$ & $100.0 \%$ & $100.0 \%$ \\
\hline
\end{tabular}

Table 172. Nadap blade assemblage (complete specimens) platform type frequency by raw material

\begin{tabular}{|c|c|c|c|c|c|}
\hline & \multicolumn{2}{|c|}{ Raw material } & \multirow{3}{*}{$\begin{array}{c}\text { Total } \\
82\end{array}$} \\
\hline & & & regional & transcarpathian & \\
\hline \multirow[t]{10}{*}{ Platform } & \multirow[t]{2}{*}{ plain } & count & 24 & 58 & \\
\hline & & \% within raw material & $96.0 \%$ & $81.7 \%$ & $85.4 \%$ \\
\hline & \multirow[t]{2}{*}{ dihedral } & count & 0 & 3 & 3 \\
\hline & & $\%$ within raw material & $0.0 \%$ & $4.2 \%$ & $3.1 \%$ \\
\hline & \multirow[t]{2}{*}{ faceted } & count & 0 & 6 & 6 \\
\hline & & $\%$ within raw material & $0.0 \%$ & $8.5 \%$ & $6.3 \%$ \\
\hline & \multirow[t]{2}{*}{ linear } & count & 1 & 3 & 4 \\
\hline & & $\%$ within raw material & $4.0 \%$ & $4.2 \%$ & $4.2 \%$ \\
\hline & \multirow[t]{2}{*}{ irregular } & count & 0 & 1 & 1 \\
\hline & & $\%$ within raw material & $0.0 \%$ & $1.4 \%$ & $1.0 \%$ \\
\hline \multirow{2}{*}{\multicolumn{2}{|c|}{ Total }} & count & 25 & 71 & 96 \\
\hline & & $\%$ within raw material & $100.0 \%$ & $100.0 \%$ & $100.0 \%$ \\
\hline
\end{tabular}

Table 173. Nadap blade assemblage (complete specimens) impact point-overhang co-presence by raw material

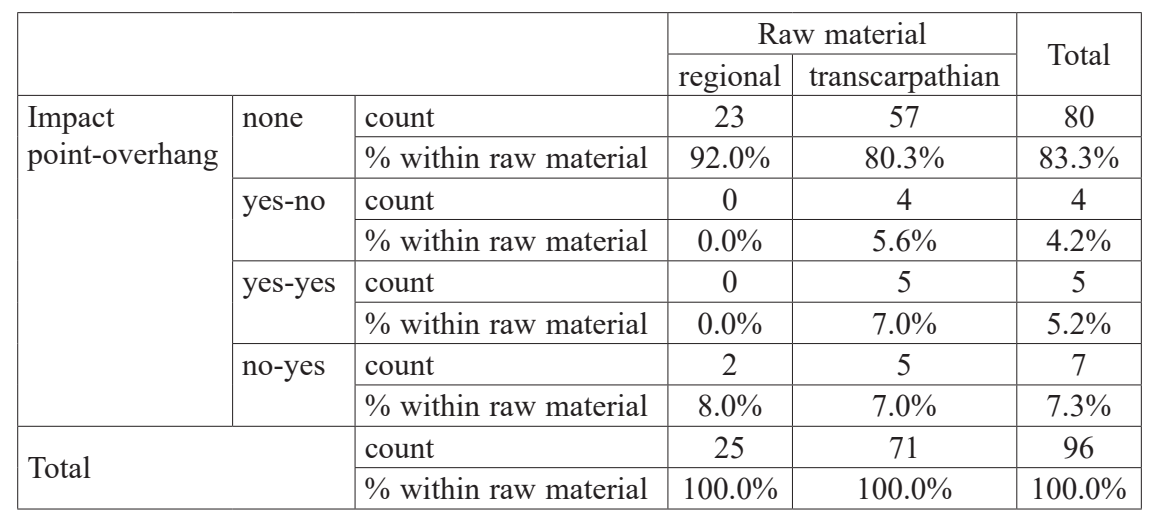


Table 174. Nadap blade assemblage (complete specimens) length, width and thickness by raw material

\begin{tabular}{|c|c|c|c|c|}
\hline \multicolumn{2}{|l|}{ Raw material } & Length $[\mathrm{mm}]$ & Width [mm] & Thickness [mm] \\
\hline \multirow[t]{6}{*}{ Regional } & Minimum & 27.00 & 8.90 & 2.80 \\
\hline & Maximum & 75.90 & 31.90 & 8.70 \\
\hline & Mean & 46.8640 & 16.7200 & 5.1000 \\
\hline & Median & 45.1000 & 14.8000 & 4.8000 \\
\hline & $\mathrm{N}$ & 25 & 25 & 25 \\
\hline & Std. deviation & 14.90785 & 5.49788 & 1.70514 \\
\hline \multirow[t]{6}{*}{ Transcarpathian } & Minimum & 19.00 & 8.20 & 1.80 \\
\hline & Maximum & 99.40 & 28.09 & 17.05 \\
\hline & Mean & 45.0428 & 16.3897 & 5.5804 \\
\hline & Median & 41.7400 & 16.5000 & 4.9000 \\
\hline & $\mathrm{N}$ & 71 & 71 & 71 \\
\hline & Std. deviation & 15.78441 & 4.64617 & 2.77533 \\
\hline \multirow[t]{6}{*}{ Total } & Minimum & 19.00 & 8.20 & 1.80 \\
\hline & Maximum & 99.40 & 31.90 & 17.05 \\
\hline & Mean & 45.5171 & 16.4757 & 5.4553 \\
\hline & Median & 42.1500 & 15.8000 & 4.8500 \\
\hline & $\mathrm{N}$ & 96 & 96 & 96 \\
\hline & Std. deviation & 15.50400 & 4.85423 & 2.54066 \\
\hline
\end{tabular}

Table 175. Nadap blade assemblage (complete specimens) t-test to compare length, width and thickness by raw materials

\begin{tabular}{|c|c|c|c|c|c|c|c|}
\hline \multicolumn{8}{|c|}{ Independent Samples Test } \\
\hline & \multicolumn{2}{|c|}{$\begin{array}{l}\text { Levene's Test } \\
\text { for Equality } \\
\text { of Variances }\end{array}$} & \multicolumn{5}{|c|}{ t-test for Equality of Means } \\
\hline & $\mathrm{F}$ & Sig. & $\mathrm{t}$ & df & Sig. (2-tailed) & $\begin{array}{c}\text { Mean } \\
\text { difference }\end{array}$ & $\begin{array}{l}\text { Std. error } \\
\text { difference }\end{array}$ \\
\hline \multirow{2}{*}{$\begin{array}{l}\text { Length } \\
{[\mathrm{mm}]}\end{array}$} & 0.003 & 0.958 & 0.503 & 94 & 0.616 & 1.82118 & 3.61988 \\
\hline & & & 0.517 & 44.319 & 0.608 & 1.82118 & 3.52120 \\
\hline \multirow{2}{*}{$\begin{array}{l}\text { Width } \\
{[\mathrm{mm}]}\end{array}$} & 1.187 & 0.279 & 0.291 & 94 & 0.772 & 0.33028 & 1.13438 \\
\hline & & & 0.269 & 36.790 & 0.790 & 0.33028 & 1.23008 \\
\hline \multirow{2}{*}{$\begin{array}{l}\text { Thickness } \\
{[\mathrm{mm}]}\end{array}$} & 3.608 & 0.061 & -0.812 & 94 & 0.419 & -0.48042 & 0.59192 \\
\hline & & & -1.013 & 69.056 & 0.314 & -0.48042 & 0.47412 \\
\hline
\end{tabular}

Table 176. Nadap tool assemblage product composition by raw materials

\begin{tabular}{|l|l|c|c|c|}
\hline \multicolumn{2}{|c|}{} & \multicolumn{2}{|c|}{ Raw material } & \multirow{2}{*}{ Total } \\
\cline { 3 - 5 } \multicolumn{2}{|c|}{ Flake } & regional & transcarpathian & \\
\cline { 2 - 5 } & count & 1 & 3 & 4 \\
\hline \multirow{3}{*}{ Blade } & count & 14 & $4.9 \%$ & $6.1 \%$ \\
\cline { 2 - 5 } & $\%$ within raw material & $6.7 \%$ & 48 & 62 \\
\hline \multirow{2}{*}{ Total } & count & $93.3 \%$ & $94.1 \%$ & $93.9 \%$ \\
\cline { 2 - 5 } & $\%$ within raw material & $100.0 \%$ & $100.0 \%$ & 66 \\
\hline
\end{tabular}


Table 177. Nadap knapped product frequency in tool assemblage by raw materials

\begin{tabular}{|c|c|c|c|c|c|c|}
\hline & \multicolumn{2}{|c|}{ Raw material } & \multirow{2}{*}{ Total } \\
\hline & & & & regional & transcarpathian & \\
\hline \multirow[t]{6}{*}{ Flake } & \multirow[t]{4}{*}{ state } & \multirow[t]{2}{*}{ blank } & count & 29 & 79 & 108 \\
\hline & & & $\%$ within raw material & $96.7 \%$ & $96.3 \%$ & $96.4 \%$ \\
\hline & & \multirow[t]{2}{*}{ tool } & count & 1 & 3 & 4 \\
\hline & & & $\%$ within raw material & $3.3 \%$ & $3.7 \%$ & $3.6 \%$ \\
\hline & \multirow{2}{*}{\multicolumn{2}{|c|}{ total }} & count & 30 & 82 & 112 \\
\hline & & & $\%$ within raw material & $100.0 \%$ & $100.0 \%$ & $100.0 \%$ \\
\hline \multirow[t]{6}{*}{ Blade } & \multirow[t]{4}{*}{ state } & \multirow[t]{2}{*}{ blank } & count & 142 & 357 & 499 \\
\hline & & & $\%$ within raw material & $91.0 \%$ & $88.1 \%$ & $88.9 \%$ \\
\hline & & \multirow[t]{2}{*}{ tool } & count & 14 & 48 & 62 \\
\hline & & & $\%$ within raw material & $9.0 \%$ & $11.9 \%$ & $11.1 \%$ \\
\hline & \multirow{2}{*}{\multicolumn{2}{|c|}{ total }} & count & 156 & 405 & 561 \\
\hline & & & $\%$ within raw material & $100.0 \%$ & $100.0 \%$ & $100.0 \%$ \\
\hline
\end{tabular}

Table 178. Nadap blade tool assemblage length, width and thickness by raw materials

\begin{tabular}{|c|c|c|c|c|}
\hline \multicolumn{2}{|l|}{ Raw material } & Length $[\mathrm{mm}]$ & Width [mm] & Thickness [mm] \\
\hline \multirow[t]{6}{*}{ Regional } & Minimum & 9.70 & 5.90 & 2.10 \\
\hline & Maximum & 65.60 & 21.00 & 5.20 \\
\hline & Mean & 30.4714 & 10.8000 & 3.3643 \\
\hline & Median & 28.9500 & 9.3000 & 3.2500 \\
\hline & $\mathrm{N}$ & 14 & 14 & 14 \\
\hline & Std. deviation & 15.94763 & 4.42354 & 0.81958 \\
\hline \multirow[t]{6}{*}{ Transcarpathian } & Minimum & 7.90 & 4.90 & 1.40 \\
\hline & Maximum & 87.10 & 32.20 & 18.90 \\
\hline & Mean & 31.5190 & 12.1494 & 5.0117 \\
\hline & Median & 26.6200 & 9.1500 & 3.6000 \\
\hline & $\mathrm{N}$ & 48 & 48 & 48 \\
\hline & Std. deviation & 16.69667 & 6.82689 & 3.74654 \\
\hline \multirow[t]{6}{*}{ Total } & Minimum & 7.90 & 4.90 & 1.40 \\
\hline & Maximum & 87.10 & 32.20 & 18.90 \\
\hline & Mean & 31.2824 & 11.8447 & 4.6397 \\
\hline & Median & 27.0700 & 9.2500 & 3.5000 \\
\hline & $\mathrm{N}$ & 62 & 62 & 62 \\
\hline & Std. deviation & 16.40709 & 6.35638 & 3.38237 \\
\hline
\end{tabular}


Table 179. Nadap blade blank and tool length, width and thickness mean t-test comparison by raw material

\begin{tabular}{|c|c|c|c|c|c|c|c|c|}
\hline \multicolumn{9}{|c|}{ Independent Samples Test } \\
\hline \multirow{2}{*}{\multicolumn{2}{|c|}{ Raw material }} & $\begin{array}{l}\text { Leven } \\
\text { for } \mathrm{E} \\
\text { of } \mathrm{Va}\end{array}$ & $\begin{array}{l}\text { s Test } \\
\text { uality } \\
\text { ances }\end{array}$ & \multicolumn{5}{|c|}{ t-test for Equality of Means } \\
\hline & & $\mathrm{F}$ & Sig. & $\mathrm{t}$ & df & $\begin{array}{c}\text { Sig. } \\
\text { (2-tailed) }\end{array}$ & $\begin{array}{c}\text { Mean } \\
\text { difference }\end{array}$ & $\begin{array}{l}\text { Std. error } \\
\text { difference }\end{array}$ \\
\hline \multirow[t]{6}{*}{ Regional } & \multirow{2}{*}{$\begin{array}{l}\text { length } \\
{[\mathrm{mm}]}\end{array}$} & 0.005 & 0.946 & 3.214 & 37 & 0.003 & 16.39257 & 5.10102 \\
\hline & & & & 3.151 & 25.525 & 0.004 & 16.39257 & 5.20153 \\
\hline & \multirow{2}{*}{$\begin{array}{l}\text { width } \\
{[\mathrm{mm}]}\end{array}$} & 1.879 & 0.179 & 3.446 & 37 & 0.001 & 5.92000 & 1.71779 \\
\hline & & & & 3.667 & 32.177 & 0.001 & 5.92000 & 1.61455 \\
\hline & \multirow{2}{*}{$\begin{array}{l}\text { thickness } \\
{[\mathrm{mm}]}\end{array}$} & 12.106 & 0.001 & 3.570 & 37 & 0.001 & 1.73571 & 0.48626 \\
\hline & & & & 4.282 & 36.438 & 0.000 & 1.73571 & 0.40531 \\
\hline \multirow[t]{6}{*}{ Transcarpathian } & \multirow{2}{*}{$\begin{array}{l}\text { length } \\
{[\mathrm{mm}]}\end{array}$} & 0.098 & 0.754 & 4.479 & 117 & 0.000 & 13.52386 & 3.01916 \\
\hline & & & & 4.431 & 97.142 & 0.000 & 13.52386 & 3.05238 \\
\hline & \multirow{2}{*}{$\begin{array}{l}\text { width } \\
{[\mathrm{mm}]}\end{array}$} & 7.798 & 0.006 & 4.034 & 117 & 0.000 & 4.24034 & 1.05105 \\
\hline & & & & 3.755 & 76.037 & 0.000 & 4.24034 & 1.12916 \\
\hline & \multirow{2}{*}{$\begin{array}{l}\text { thickness } \\
{[\mathrm{mm}]}\end{array}$} & 1.117 & 0.293 & 0.951 & 117 & 0.344 & 0.56876 & 0.59817 \\
\hline & & & & 0.898 & 80.868 & 0.372 & 0.56876 & 0.63318 \\
\hline
\end{tabular}

Table 180. Nadap blade tool length, width and thickness mean comparison by raw materials with t-test

\begin{tabular}{|l|c|c|c|c|c|c|c|}
\hline \multicolumn{1}{|c|}{} & $\begin{array}{c}\text { Levene's Test } \\
\text { for Equality } \\
\text { of Variances }\end{array}$ & \multicolumn{7}{|c|}{ Independent Samples Test } \\
\cline { 2 - 8 } & $\mathrm{F}$ & Sig. & $\mathrm{t}$ & $\mathrm{df}$ & Sig. (2-tailed) & $\begin{array}{c}\text { Mean } \\
\text { difference }\end{array}$ & $\begin{array}{c}\text { Std. error } \\
\text { difference }\end{array}$ \\
\hline \multirow{2}{*}{$\begin{array}{l}\text { Length } \\
{[\mathrm{mm}]}\end{array}$} & 0.046 & 0.831 & -0.209 & 60 & 0.836 & -1.04753 & 5.02313 \\
\hline $\begin{array}{l}\text { Width } \\
{[\mathrm{mm}]}\end{array}$ & 4.214 & 0.044 & -0.696 & 60 & 0.489 & -1.34937 & 4.89633 \\
\hline $\begin{array}{l}\text { Thickness } \\
{[\mathrm{mm}]}\end{array}$ & 7.440 & 0.008 & -1.625 & 60 & 0.109 & -1.64738 & 1.93894 \\
\cline { 2 - 8 } & & & -2.824 & 58.039 & 0.006 & -1.64738 & 0.53834 \\
\hline
\end{tabular}

Table 181. Nadap blade tool types by raw material

\begin{tabular}{|c|c|c|c|c|c|}
\hline & \multicolumn{2}{|c|}{ Raw material } & \multirow{2}{*}{ Total } \\
\hline & & & regional & transcarpathian & \\
\hline \multirow[t]{8}{*}{ Tooltypes } & \multirow[t]{2}{*}{ end-scraper } & count & 0 & 4 & 4 \\
\hline & & $\%$ within raw material & $0.0 \%$ & $8.3 \%$ & $6.5 \%$ \\
\hline & \multirow[t]{2}{*}{ burin } & count & 0 & 10 & 10 \\
\hline & & $\%$ within raw material & $0.0 \%$ & $20.8 \%$ & $16.1 \%$ \\
\hline & \multirow[t]{2}{*}{ truncation } & count & 0 & 1 & 1 \\
\hline & & $\%$ within raw material & $0.0 \%$ & $2.1 \%$ & $1.6 \%$ \\
\hline & \multirow[t]{2}{*}{ armature } & count & 14 & 33 & 47 \\
\hline & & $\%$ within raw material & $100.0 \%$ & $68.8 \%$ & $75.8 \%$ \\
\hline \multirow{2}{*}{\multicolumn{2}{|c|}{ Total }} & count & 14 & 48 & 62 \\
\hline & & $\%$ within raw material & $100.0 \%$ & $100.0 \%$ & $100.0 \%$ \\
\hline
\end{tabular}


Table 182. Nadap armature types by raw material

\begin{tabular}{|l|l|l|c|c|c|}
\hline \multicolumn{2}{|c|}{} & \multicolumn{2}{|c|}{ Raw material } & \multirow{2}{*}{ Total } \\
\cline { 3 - 6 } \multicolumn{2}{|c|}{ Armatures } & backed & regional & transcarpathian & \\
\cline { 3 - 6 } & & $\%$ within raw material & $78.6 \%$ & $75.8 \%$ & $76.6 \%$ \\
\cline { 2 - 6 } & $\begin{array}{l}\text { backed- } \\
\text { truncated }\end{array}$ & count & 1 & 5 & 6 \\
\cline { 3 - 6 } & \multirow{2}{*}{ points } & \% within raw material & $7.1 \%$ & $15.2 \%$ & $12.8 \%$ \\
\cline { 3 - 6 } & & \%ount & 2 & 3 & 5 \\
\hline \multirow{2}{*}{ Total } & count & $14.3 \%$ & $9.1 \%$ & $10.6 \%$ \\
\cline { 3 - 6 } & \% within raw material & $100.0 \%$ & $100.0 \%$ & $100.0 \%$ \\
\hline
\end{tabular}

Table 183. Nadap point types by raw material

\begin{tabular}{|l|l|l|c|c|c|}
\hline \multicolumn{2}{|c|}{} & \multicolumn{2}{|c|}{ Raw material } & \multirow{2}{*}{ Total } \\
\cline { 3 - 6 } \multicolumn{2}{|c|}{ Points } & backed & regional & transcarpathian & \\
\cline { 3 - 6 } & & $\%$ within raw material & $50.0 \%$ & $33.3 \%$ & $40.0 \%$ \\
\cline { 3 - 6 } & $\begin{array}{l}\text { arched } \\
\text { backed }\end{array}$ & count & 0 & 1 & 1 \\
\cline { 2 - 6 } & $\begin{array}{l}\text { curved } \\
\text { backed }\end{array}$ & count & 1 & 1 & $20.0 \%$ \\
\cline { 3 - 6 } & & $\%$ within raw material & $0.0 \%$ & $33.3 \%$ & 2 \\
\hline \multirow{2}{*}{ Total } & count & $50.0 \%$ & $33.3 \%$ & $40.0 \%$ \\
\cline { 3 - 6 } & \% within raw material & $100.0 \%$ & $100.0 \%$ & $100.0 \%$ \\
\hline
\end{tabular}

Table 184. Nadap flake assemblage (complete specimens) dorsal scar pattern frequency by raw material

\begin{tabular}{|c|c|c|c|c|c|}
\hline & \multicolumn{2}{|c|}{ Raw material } & \multirow{3}{*}{$\begin{array}{c}\text { Total } \\
11\end{array}$} \\
\hline & & & regional & transcarpathian & \\
\hline \multirow[t]{10}{*}{ Scars } & \multirow[t]{2}{*}{ unidirectional } & count & 2 & 9 & \\
\hline & & $\%$ within raw material & $18.2 \%$ & $42.9 \%$ & $34.4 \%$ \\
\hline & \multirow[t]{2}{*}{ opposite } & count & 2 & 1 & 3 \\
\hline & & $\%$ within raw material & $18.2 \%$ & $4.8 \%$ & $9.4 \%$ \\
\hline & \multirow[t]{2}{*}{ perpendicular } & count & 6 & 8 & 14 \\
\hline & & $\%$ within raw material & $54.5 \%$ & $38.1 \%$ & $43.8 \%$ \\
\hline & \multirow[t]{2}{*}{ multiple } & count & 1 & 2 & 3 \\
\hline & & $\%$ within raw material & $9.1 \%$ & $9.5 \%$ & $9.4 \%$ \\
\hline & \multirow[t]{2}{*}{ no scar } & count & 0 & 1 & 1 \\
\hline & & $\%$ within raw material & $0.0 \%$ & $4.8 \%$ & $3.1 \%$ \\
\hline \multirow{2}{*}{\multicolumn{2}{|c|}{ Total }} & count & 11 & 21 & 32 \\
\hline & & $\%$ within raw material & $100.0 \%$ & $100.0 \%$ & $100.0 \%$ \\
\hline
\end{tabular}


Table 185. Nadap flake assemblage (complete specimens) platform type frequency by raw material

\begin{tabular}{|c|c|c|c|c|c|}
\hline & \multicolumn{2}{|c|}{ Raw material } & \multirow{2}{*}{ Total } \\
\hline & & & regional & transcarpathian & \\
\hline \multirow[t]{8}{*}{ Platform } & \multirow[t]{2}{*}{ plain } & count & 10 & 15 & 25 \\
\hline & & $\%$ within raw material & $90.9 \%$ & $71.4 \%$ & $78.1 \%$ \\
\hline & \multirow[t]{2}{*}{ dihedral } & count & 1 & 2 & 3 \\
\hline & & $\%$ within raw material & $9.1 \%$ & $9.5 \%$ & $9.4 \%$ \\
\hline & \multirow[t]{2}{*}{ faceted } & count & 0 & 2 & 2 \\
\hline & & $\%$ within raw material & $0.0 \%$ & $9.5 \%$ & $6.3 \%$ \\
\hline & \multirow[t]{2}{*}{ cortical } & count & 0 & 2 & 2 \\
\hline & & $\%$ within raw material & $0.0 \%$ & $9.5 \%$ & $6.3 \%$ \\
\hline \multirow{2}{*}{\multicolumn{2}{|c|}{ Total }} & count & 11 & 21 & 32 \\
\hline & & $\%$ within raw material & $100.0 \%$ & $100.0 \%$ & $100.0 \%$ \\
\hline
\end{tabular}

Table 186. Nadap flake assemblage (complete specimens) impact point-overhang frequency by raw material

\begin{tabular}{|c|c|c|c|c|c|}
\hline & \multicolumn{2}{|c|}{ Raw material } & \multirow{2}{*}{ Total } \\
\hline & & & regional & transcarpathian & \\
\hline \multirow{8}{*}{$\begin{array}{l}\text { Impact } \\
\text { point-overhang }\end{array}$} & \multirow[t]{2}{*}{ none } & count & 3 & 5 & 8 \\
\hline & & $\%$ within raw material & $27.3 \%$ & $23.8 \%$ & $25.0 \%$ \\
\hline & \multirow[t]{2}{*}{ yes-no } & count & 3 & 2 & 5 \\
\hline & & $\%$ within raw material & $27.3 \%$ & $9.5 \%$ & $15.6 \%$ \\
\hline & \multirow[t]{2}{*}{ yes-yes } & count & 3 & 9 & 12 \\
\hline & & $\%$ within raw material & $27.3 \%$ & $42.9 \%$ & $37.5 \%$ \\
\hline & \multirow[t]{2}{*}{ no-yes } & count & 2 & 5 & 7 \\
\hline & & $\%$ within raw material & $18.2 \%$ & $23.8 \%$ & $21.9 \%$ \\
\hline \multirow{2}{*}{\multicolumn{2}{|c|}{ Total }} & count & 11 & 21 & 32 \\
\hline & & \% within raw material & $100.0 \%$ & $100.0 \%$ & $100.0 \%$ \\
\hline
\end{tabular}

Table 187. Nadap flake blank assemblage length, width and thickness by raw materials

\begin{tabular}{|c|c|c|c|c|}
\hline \multicolumn{2}{|l|}{ Raw material } & Length [mm] & Width [mm] & Thickness [mm] \\
\hline \multirow[t]{6}{*}{ Regional } & Minimum & 14.60 & 9.70 & 2.20 \\
\hline & Maximum & 40.50 & 30.30 & 8.20 \\
\hline & Mean & 25.6455 & 22.4455 & 4.9909 \\
\hline & Median & 21.3000 & 22.2000 & 4.2000 \\
\hline & $\mathrm{N}$ & 11 & 11 & 11 \\
\hline & Std. deviation & 10.02161 & 6.21375 & 1.92377 \\
\hline \multirow[t]{6}{*}{ Transcarpathian } & Minimum & 10.00 & 10.20 & 2.80 \\
\hline & Maximum & 52.12 & 42.60 & 9.27 \\
\hline & Mean & 24.7890 & 22.1071 & 6.0486 \\
\hline & Median & 22.7000 & 21.1900 & 6.5000 \\
\hline & $\mathrm{N}$ & 21 & 21 & 21 \\
\hline & Std. deviation & 9.52437 & 7.18257 & 2.10816 \\
\hline \multirow[t]{6}{*}{ Total } & Minimum & 10.00 & 9.70 & 2.20 \\
\hline & Maximum & 52.12 & 42.60 & 9.27 \\
\hline & Mean & 25.0834 & 22.2234 & 5.6850 \\
\hline & Median & 22.0000 & 21.5500 & 5.7500 \\
\hline & $\mathrm{N}$ & 32 & 32 & 32 \\
\hline & Std. deviation & 9.54428 & 6.76500 & 2.07886 \\
\hline
\end{tabular}


Lithic analysis of the Middle and Late Upper Palaeolithic in Hungary

Table 188. Nadap flake blank length, width and thickness mean comparison by raw materials with t-test

\begin{tabular}{|l|c|c|c|c|c|c|c|}
\hline \multicolumn{1}{|c|}{} & $\begin{array}{l}\text { Levene's Test } \\
\text { for Equality } \\
\text { of Variances }\end{array}$ & \multicolumn{7}{|c|}{ Independent Samples Test } \\
\cline { 2 - 8 } & $\mathrm{F}$ & Sig. & $\mathrm{t}$ & $\mathrm{df}$ & Sig. (2-tailed) & $\begin{array}{c}\text { Mean } \\
\text { difference }\end{array}$ & $\begin{array}{c}\text { Std. error } \\
\text { difference }\end{array}$ \\
\hline \multirow{2}{*}{$\begin{array}{l}\text { Length } \\
{[\mathrm{mm}]}\end{array}$} & 1.044 & 0.315 & 0.237 & 30 & 0.814 & 0.85641 & 3.60765 \\
\hline & & & 0.234 & 19.516 & 0.818 & 0.85641 & 3.66742 \\
\hline $\begin{array}{l}\text { Width } \\
{[\mathrm{mm}]}\end{array}$ & 0.002 & 0.967 & 0.132 & 30 & 0.896 & 0.33831 & 2.55876 \\
\hline $\begin{array}{l}\text { Thickness } \\
{[\mathrm{mm}]}\end{array}$ & 0.215 & 0.647 & -1.387 & 30 & 0.176 & -1.05766 & 0.76246 \\
\cline { 2 - 8 } & & & -1.429 & 22.154 & 0.167 & -1.05766 & 0.74033 \\
\hline
\end{tabular}

Table 189. Nadap flake blank and tool length, width and thickness mean t-test comparison by raw material

\begin{tabular}{|c|c|c|c|c|c|c|c|}
\hline \multirow{3}{*}{ Raw material } & $\begin{array}{c}\text { Levene's Test } \\
\text { for Equality } \\
\text { of Variances }\end{array}$ & \multicolumn{7}{|c|}{ Independent Samples Test } \\
\cline { 2 - 8 } & $\mathrm{F}$ & Sig. & $\mathrm{t}$ & $\mathrm{df}$ & Sig. (2-tailed) & $\begin{array}{c}\text { Mean } \\
\text { difference }\end{array}$ & $\begin{array}{c}\text { Std. error } \\
\text { difference }\end{array}$ \\
\hline Transcarpathian & 0.037 & 0.850 & -0.859 & 22 & 0.400 & -5.05095 & 5.88031 \\
\cline { 2 - 8 } & & & -0.857 & 2.603 & 0.463 & -5.05095 & 5.89536 \\
\cline { 2 - 8 } & 0.020 & 0.888 & 1.289 & 22 & 0.211 & 5.62381 & 4.36201 \\
\cline { 2 - 8 } & & & 1.523 & 2.962 & 0.226 & 5.62381 & 3.69169 \\
\cline { 2 - 8 } & 6.340 & 0.020 & -2.369 & 22 & 0.027 & -3.55143 & 1.49901 \\
\cline { 2 - 8 } & & & -1.340 & 2.126 & 0.306 & -3.55143 & 2.65047 \\
\hline
\end{tabular}

Table 190. Nadap flake tool types by raw material

\begin{tabular}{|l|l|l|c|c|c|}
\hline \multicolumn{2}{|c|}{} & \multicolumn{2}{|c|}{ Raw material } & \multirow{2}{*}{ Total } \\
\cline { 3 - 6 } \multicolumn{1}{|c|}{ Tooltypes } & \multirow{2}{*}{ endscraper } & reunt & 1 & 0 & 1 \\
\cline { 3 - 6 } & & $\%$ within raw material & $100.0 \%$ & $0.0 \%$ & $25.0 \%$ \\
\cline { 3 - 6 } & \multirow{3}{*}{ burin } & count & 0 & 1 & 1 \\
\cline { 3 - 6 } & & $\%$ within raw material & $0.0 \%$ & $33.3 \%$ & $25.0 \%$ \\
\cline { 3 - 6 } & \multirow{2}{*}{ retouched } & count & 0 & 1 & 1 \\
\cline { 3 - 6 } & & $\%$ within raw material & $0.0 \%$ & $33.3 \%$ & $25.0 \%$ \\
\cline { 3 - 6 } & truncation & count & 0 & 1 & 1 \\
\cline { 3 - 6 } & & $\%$ within raw material & $0.0 \%$ & $33.3 \%$ & $25.0 \%$ \\
\hline \multirow{2}{*}{ Total } & count & 1 & 3 & 4 \\
\cline { 3 - 6 } & $\%$ within raw material & $100.0 \%$ & $100.0 \%$ & $100.0 \%$ \\
\hline
\end{tabular}




\section{CONSIDERATIONS ON TECHNOLOGICAL DATA}

\section{Raw materials}

The technological data provided here showed that local raw material procurement was dominant solely in the Late Gravettian (LG) and only at two sites, Arka and Bodrogkeresztúr. Further sites of LG, also the Early Epigravettian (EE) camps, exploited regional sources, making this strategy the most common in the MUP and LUP. In contrast, the Late Epigravettian (LE) lithic procurement relied mostly on transcarpathian sources (TC). Latter case is an unusual strategy because regional lithic raw material sources in the Carpathian basin always were available. The abundance of TC material in LE is rather just apparently unique, because Arka and Bodrogkeresztúr comprise $1835 \mathrm{~g}$ and $1739 \mathrm{~g}$ of TC material, respectively, which are higher than the $1702 \mathrm{~g}$ at Nadap, and just slightly lower than the $2334 \mathrm{~g}$ at Esztergom. This means that possessing approximately $2 \mathrm{~kg}$ of TC material in LE is not unusual in the archaeological record of the Carpathian basin. The uniqueness of LE assemblages, indeed, is the low percentage of regional materials.

The frequency of the lithic raw material types were tested with K-mean cluster analysis to see if the raw material procurement areas can be linked with the archaeological period. The analysis made one cluster for Corvin-tér, Hidasnémeti, Ságvár and Sajószentpéter; another for Arka and Bodrogkeresztúr; and a third for Nadap and Esztergom. The first cluster corresponds with assemblages containing no or very low frequency of TC material. Cluster two have a considerable but not dominating frequency of TC material. Cluster three is composed of those assemblages dominated by TC material. These three clusters do not fit completely the three chronological panels. Only TC material dominance may identify LE assemblages.

The technological analyses showed that determining the dominant raw material processed at a site after the number of knapped items sometimes might be misleading. For example, Corvin-tér yielded most of its knapped products from regional radiolarite material, but the locally available coarse grain material with smaller number outweighs the regional material. Leaving the mass of the local material unworked seems to be related with its coarser quality, which is less apt for fine tool production.

The lower rate of local material fragmentation, nonetheless, seems to be a very general phenomenon. When local material is available in large quantity, the number of products per one kilogram of the raw material is always lower compared to other materials. At Arka, Bodrogkeresztúr and Corvin-tér, 60-64 items were produced from one kilogram of local material, while the regional material yield was at least twice greater, and TC material always yielded the greatest number of artifact per kilogram. TC yield was also high at Nadap and Esztergom, 431 and 505 items, respectively. These observations showed that the greater the distance between the site and the lithic source, the greater the reduction of the raw material. 


\section{The blade production}

The most common debitage product in the MUP and LUP is the blade. The blade component of a toolkit in most cases is greater than $50 \%$, which also reflects the importance of blade production. Out of the studied assemblages, the LE sites have the highest blade portions. Blade frequency, therefore, seemingly correlates with the threefold chronological division of the assemblages by the Gravettian Entity Model (GEM), which means that out of the three periods the smallest amount of blades was produced by EE, the greatest ratio by LE, and a moderate ratio by LG. The drop of blade proportion in the EE assemblages on the expense of the flakes in comparison with LG assemblages, however, is not significant $\left(\mathrm{r}_{\mathrm{s}}=-0.621, \mathrm{n}=6, \mathrm{p}=0.188\right)$. This is most likely due to that local raw material processing, which is characteristic only to two LG sites, produces a vast number of flakes which pulls down overall blade ratio. When local raw materials are excluded from the correlation test, the decrease of blade ratio from $L G$ to $E E$ becomes significant $\left(r_{s}=-0.828, n=6, p=0.042\right)$. The low blade production rate in EE also is demonstrable in comparison with LE $\left(\mathrm{r}_{\mathrm{s}}=0.961\right.$, $\mathrm{n}=6, \mathrm{p}=0.039$ ). The low blade frequency therefore can be a marker of EE lithic assemblages.

Considering the blade yield by raw materials, local and regional materials regularly produced a lower rate compared to TC. This is the case at Arka, Bodrogkeresztúr, Esztergom and Hidasnémeti. Nadap and Ságvár are outliers of this tendency with making a greater percent of blades from regional material. Therefore, the raw material consumption by blade debitage seems to increase parallel with the distance between the site and the lithic source, similarly to the growth of the reduction intensity.

At Arka and Bodrogkeresztúr, the blade yield from the total weight of local raw material is $11.16 \%$ and $11.29 \%$, of regional material is $16.11 \%$ and $21.81 \%$, and of TC material is $39.5 \%$ and $68.1 \%$, respectively. At Esztergom, $71.4 \%$ of all TC material is blade by weight, and at Nadap this is $53.6 \%$. These percentages showed that TC materials in any case were consumed chiefly for blade production. This can be observed even at Ságvár through 2-3\% blade yield from the regional and distant materials, and $9.52 \%$ from TC materials. The outstandingly high blade yield at LE sites, however, could also be related with the high frequency of armatures, the fabrication of which requires merely blades.

The blade knapping modality did not show clear differences between the assemblages. The two basic methods studied here, the single platform and double platform reduction, did not seem to correlate neither with raw material type nor period. Unidirectional blade exploitation was the most common, and except for Corvin-tér and Esztergom, the assemblages contain double platform cores in varying frequency. Nadap is the only site with double platform core dominance, and this is the sole assemblage with the lowest ratio of unidirectional scars on blades. Testing the correlation between unidirectional scar frequency on blades and the chronology of the assemblages found no relationship regarding neither $L G$ and $E E\left(r_{s}=-0.587, n=6\right.$, $\mathrm{p}=0.220)$ nor $\mathrm{EE}$ and $\operatorname{LE}\left(\mathrm{r}_{\mathrm{s}}=-0.236, \mathrm{n}=4, \mathrm{p}=0.764\right)$. The failed correlation and 
the different ratio of unidirectional versus bidirectional reduction between Nadap and Esztergom demonstrates the blade debitage modalities were culturally unfixed.

The blade dimension is also an unstable variable $\left(\mathrm{F}_{(7,1134)}=30.003, \mathrm{p}<0.001\right)$. EE assemblages contain the shortest blades and these are the only ones which are not similar to any other blade assemblage according to the post hoc test (Tab. 191). However, Arka, while being similar to Bodrogkeresztúr and Sajószentpéter, is also similar to the two LE sites, Esztergom and Nadap, and differs from the contemporaneous Hidasnémeti, which is, in turn, similar to the LE Esztergom and the LG Sajószentpéter. Comparing the blade lengths by the three age clusters, the longest blades were made in LG and the shortest in EE assemblages. ANOVA $\left(\mathrm{F}_{(2,1139)}=93.264, \mathrm{p}<0.001\right)$ found these differences significant. But, LE blades also differ from the other two groups, by being shorter than LG and longer than EE (Tab. 192).

Studying the blade length by raw material within LG assemblages showed that Arka made longer local blades in average than Bodrogkeresztúr $\left(\mathrm{t}_{(445.584)}=3.279, \mathrm{p}=0.002\right)$. Comparing regional blades from Arka, Bodrogkeresztúr, Hidasnémeti and Sajószentpéter, the means are also different $\left(\mathrm{F}_{(3,220)}=18.538, \mathrm{p}<0.001\right)$ because Hidasnémeti produced the longest blades, but the lengths of the other three LG assemblages are similar (Tab. 193). Comparing TC blades from Arka and Bodrogkeresztúr, there is no difference in blade length $\left(\mathrm{t}_{(101)}=0.679, \mathrm{p}=0.499\right)$. Within $\mathrm{EE}$, only the regional blade lengths can be compared, and the t-test showed that Ságvár and Corvin-tér yielded the same size $\left(\mathrm{t}_{(82.182)}=-1.508, \mathrm{p}=0.135\right)$. In LE assemblages, both the regional $\left(\mathrm{t}_{(25)}=-1.214\right.$, $\mathrm{p}=0.236)$ and the $\mathrm{TC}\left(\mathrm{t}_{(28.494)}=0.643, \mathrm{p}=0.525\right)$ blade length means are similar.

Disregarding the chronology, the blade production from local raw materials can be compared only between Arka and Bodrogkeresztúr, which has already shown differences. In the regional blade production of all assemblages, the lengths are different $\left(\mathrm{F}_{(7,423)}=31.115, \mathrm{p}<0.001\right)$. In details it means that Corvin-tér is similar to Esztergom and Ságvár, but differs from all the others, which are in turn, similar, except Hidasnémeti that differs from each assemblage, except Esztergom (Tab. 194). The TC blade material length from Arka, Bodrogkeresztúr, Nadap and Esztergom showed no differences $\left(\mathrm{F}_{(3,192)}=1.277, \mathrm{p}=0.283\right)$.

At sites yielding both local and TC raw materials, Arka and Bodrogkeresztúr, the size of the local blades are always greater than that of TC blades. This situation can be interpreted with the greater reduction of the TC material due to the long time spent in the lithic production process and use-rejuvenation-reuse cycle. But, when there is no local material (Nadap), there is no correlation between distance and blade size diminishment.

These comparisons may illustrate that the length of the blades can be highly influenced by the raw material property and the reduction intensity. Except EE sites, blade length does not decode archaeological period. This can be demonstrated with the similar mean lengths $\left(\mathrm{t}_{(377)}=-0.768, \mathrm{p}=0.443\right)$ of the local blade assemblage of Arka and the regional blade assemblage of Hidasnémeti, because the raw materials of these two blade assemblages derived from similar geological outcrops with the same properties of the knappeable rock including size and quality. Also, Ságvár showed 
that distant material blades are greater than regional blades. This is due to that most regional material for Ságvár is small sized pebble that is not eligible for producing long blades, but distant materials provided larger blocks that were apt for increasing the size of the blade products.

Comparing the thicknesses of the blades, also there are differences between the assemblages $\left(\mathrm{F}_{(7,1134)}=5.644, \mathrm{p}<0.001\right)$. Arka is the sole assemblage that differs by being constantly thicker from Corvin-tér, Nadap and Ságvár (Tab. 195). This is in accordance with Arka blades being generally larger than the average.

By raw material origin, the two local material user sites, Arka and Bodrogkeresztúr, are different $\left(\mathrm{t}_{(464.137)}=3.457, \mathrm{p}=0.001\right)$ by Arka making thicker blades. Blade thicknesses are also different $\left(\mathrm{F}_{(7,423)}=3.283, \mathrm{p}=0.002\right)$ in the regional group by Hidasnémeti being thicker than Arka, Corvin-tér and Ságvár (Tab. 196). TC material thicknesses are also different $\left(\mathrm{F}_{(3,192)}=3.576, \mathrm{p}=0.015\right)$ with Nadap blades being thicker than Bodrogkeresztúr, but these two by one by one are similar with the other two TC user assemblages, Arka and Esztergom (Tab. 197).

By chronological groups, regional blades of LG are thicker than EE blades, but EE samples are similar to LE blades (Tab. 198). Concerning TC blades, LG assemblages yielded thicker specimens than LE assemblages $\left(\mathrm{t}_{(174.900)}=-3.086, \mathrm{p}=0.002\right)$. Disregarding the raw material type, the difference in thickness is pronounced $\left(F_{(2,1139)}=16.922, p<0.001\right)$ by LG being thicker than EE and LE blades (Tab. 199).

\section{The flake production}

The flake sizes also differ among the assemblages $\left(\mathrm{F}_{(7,2369)}=117.111, \mathrm{p}<0.001\right)$. Arka differs from all other sites by making the largest flakes, and the smallest difference was found with Bodrogkeresztúr (Tab. 200). Bodrogkeresztúr is similar to Esztergom, Hidasnémeti and Sajószentpéter. Esztergom and Sajószentpéter are the only sites which differ from Arka. Comparing the flake lengths by period, the longest specimens were made by LG assemblages and the shortest ones in EE assemblages. ANOVA $\left(\mathrm{F}_{(2,2374)}=293.703, \mathrm{p}<0.001\right)$ found these differences significant. LE and EE flake sizes are similar (Tab. 201).

Studying the length of the flakes by raw materials in LG, the t-test $\left(t_{(745,986)}=9.446\right.$, $\mathrm{p}<0.001)$ showed that local flakes at Arka are longer than at Bodrogkeresztúr. Comparing the regional flakes from Arka, Bodrogkeresztúr, Hidasnémeti and Sajószentpéter, the means are similar $\left(\mathrm{F}_{(3,295)}=2.360, \mathrm{p}=0.072\right)$. The smallest difference is between Hidasnémeti and Sajószentpéter, the two assemblages which do not have local raw material production (Tab. 202). Comparing the TC flakes in LG assemblages, Arka made larger flakes than Bodrogkeresztúr $\left(\mathrm{t}_{(69.505)}=4.263, \mathrm{p}<0.001\right)$.

In $\mathrm{EE}$ assemblages, there is no difference between the lengths of the regional flakes $\left(\mathrm{t}_{(555)}=-0.650, \mathrm{p}=0.516\right)$.

In LE, there are differences between the regional flakes $\left(\mathrm{t}_{(19)}=2.932, \mathrm{p}=0.009\right)$ by Esztergom making longer flakes than Nadap. But, the TC flake size means at Esztergom and Nadap are similar $\left(\mathrm{t}_{(53.698)}=1.756, \mathrm{p}=0.085\right)$. 
Without sorting the assemblages into their chronological group, the local flake production can be compared between Arka, Bodrogkeresztúr and Corvin-tér $\left(\mathrm{F}_{(2,1155)}=41.684, \mathrm{p}<0.001\right)$. The first two assemblages have already showed differences, but Corvin-tér flakes have similar size with Bodrogkeresztúr and Arka (Tab. 203). Concerning the regional flake production, the lengths are different $\left(\mathrm{F}_{(7,869)}=26.528\right.$, $\mathrm{p}<0.001)$. Corvin-tér and Ságvár are the most dissimilar compared to the other assemblages and they are similar only to Nadap (Tab. 204). The TC flake lengths in Arka, Bodrogkeresztúr, Ságvár, Nadap and Esztergom showed differences $\left(\mathrm{F}_{(4,137)}=6.296\right.$, $\mathrm{p}<0.001$ ) with Arka being different from all but Ságvár (Tab. 205).

The flake size comparisons showed that the size of the products can be affected by the properties of the used lithic raw material. The only correlation we can see is that when local raw materials were used to make blades, the length of the flakes are greater, which is most likely due to that the locally available raw material could have been brought to the site in larger blocks, thus the blade core shaping produced greater flakes. This is in contrast with the TC materials, which usually entered the sites in smaller size, most probably as cores or smaller nodules. Therefore, the initial size of the lithic raw material seems to affect the size of the flakes. Again, comparing the flakes of Hidasnémeti and Arka, which were made of raw materials having similar properties, but the raw material is local for Arka and regional for Hidasnémeti, Arka made $15.9 \mathrm{~mm}$ longer flakes compared to Hidasnémeti and this difference is significant $\left(\mathrm{t}_{(926)}=9.378, \mathrm{p}<0.001\right)$. This supports that local raw material processing results in larger flakes as by-products of the blade technology. The same difference was found when Hidasnémeti regional flake mean length was found smaller than the Bodrogkeresztúr local flake mean length $\left(\mathrm{t}_{(344.424)}=3.796, \mathrm{p}<0.001\right)$.

Another aspect of local raw material exploitation and flake production concerns the knapping technique. Comparing the ratio of the impact point and overhang presence on the local raw material flakes at Arka and Bodrogkeresztúr with Hidasnémeti, $62.2 \%$ of the regional material flake assemblage of Hidasnémeti has impact point or unabraded overhang, while Arka and Bodrogkeresztúr flakes showed this feature reached up 89.7\% and $82.9 \%$, respectively. The hard hammer technique use in flake production generally decreases towards the production of regional, distant and TC materials $\left(\mathrm{r}_{\mathrm{s}}=-0227, \mathrm{n}=2377\right.$, $\mathrm{p}<0.001)$ and this correlation is valid for Arka, Bodrogkeresztúr and Esztergom.

\section{The tool blank selection}

Selecting the blanks for tools is quite similar between the assemblages. Ságvár is the only outlier with using more flakes than blades for tools. Although Corvint-tér has more blade tools than flake tools, these EE assemblages yielded the lowest percent of blade tools while the highest blade tool ratio is in LE assemblages. Indeed, blade tool frequency declines when LG and EE assemblages are compared $\left(\mathrm{r}_{\mathrm{s}}=-0.745\right.$, $\mathrm{n}=348, \mathrm{p}<0.001)$, and grows from EE towards LE $\left(\mathrm{r}_{\mathrm{s}}=0.836, \mathrm{n}=267, \mathrm{p}<0.001\right)$. This increase is still strong from LG to LE $\left(\mathrm{r}_{\mathrm{s}}=-0.866, \mathrm{n}=453, \mathrm{p}<0.001\right)$. The flake selection for tools is in negative relation with the blade selection. 
The ratio of raw materials in the blade toolkit diminishes with the distance to source. Bodrogkeresztúr is the only assemblage, which yielded almost equal numbers of local and TC blade tools, and Nadap and Esztergom showed a reverse order. The use of raw materials for flake tools showed the same pattern, but in the Bodrogkeresztúr flake toolkit the TC material is much fewer than the local material. Therefore, the proportion of raw materials in the toolkit mostly depends on the most frequent raw material. However, concerning the blades, there is a tendency to use up most TC items for tools, while from the local and regional material more items remained unretouched.

If we study how much of the blades from each raw material was used for tool, we see that when TC material was involved into the lithic production, the degree of blade usage for tools increases as the distance between site and raw material source grows. At Arka, 3.5 times greater the TC blade usage for tools compared to the local material, and regional blades were also used 2.9 times more to make tools than local blades. At Bodrogkeresztúr, $38.9 \%$ of all TC blades are tools, which is 3.2 times greater than the local blade usage, and regional blades were selected 2.2 times more for making tools than local blades. This pattern fits even Esztergom and Nadap where TC materials are the most frequent. Ságvár is deviant in this comparison because distant materials were less frequently selected for tools. At Corvin-tér and Sajószentpéter, this comparison is not applicable because only regional raw materials were used for making blade tools. Concerning the flakes, the same pattern can be seen: the usage rate increases as the distance grows between the site and the source. At Nadap, however, the rate is almost the same for regional and TC material (although the complete flake tool assemblage counts four items).

Local raw materials were used for tools only in two assemblages, Arka and Bodrogkeresztúr. At both sites, most local raw materials are domestic tools, and armature makes up $12.5 \%$ and $8.3 \%$ of the local material toolkit, respectively. Regional materials in Arka make up $26.7 \%$ of the armature, but it is 3.8\% in Bodrogkeresztúr, which is similar to Ságvár 3.3\%. In Esztergom and Nadap the regional materials were highly used for armature with $77.8 \%$ and $93.3 \%$. Distant materials cannot be evaluated in the same way, but at Ságvár distant materials were not used for armature, only for domestic tasks. The use of TC material is also not biased towards the armature at Arka and Bodrogkeresztúr, but in the Arka toolkit $29.5 \%$ of TC specimens is armature while at Bodrogkeresztúr this percent is 9.9. At Hidasnémeti, Ságvár and Sajószentpéter, TC material was expended only for domestic tools. At LE sites, the TC usage in armatures is the highest, $68.1 \%$ and $64.7 \%$, but since this material yielded the essence of the assemblages, and armature is the most common type, this is no surprise. Except for Esztergom and Nadap, domestic tools were mostly made of the locally available or the closest raw material types, but at Bodrogkeresztúr $44.4 \%$ of the burins were made of TC material, and the armatures were made from TC materials with $10 \%$ higher frequency than domestic tools. Correlating typological features and technological features, the frequency of blade production correlates with the frequency of the armatures in the assemblages $\left(r_{s}=0.738, n=8, p=0.037\right)$. However, the frequency of TC material has no effect on this. The use of the different raw materials therefore is not straightforwardly correlate with tool types. 
Comparing the size of the blade tools with that of the blade blanks showed that the lengths usually differ. This parameter almost always change when a blade was retouched into a tool. The width differences were less frequent and the thickness was the last that may have changed. However, for the flakes this is the very revers. The thicknesses of blank and tool specimens were commonly different, which showed that thicker items were selected for making tools among the flakes. These comparisons showed that the average blade was eligible for blade tools, and the flakes were selected according to their parameters fitting best for the type of the tool.

Comparing the sizes of the domestic tool types between the assemblages ANOVA found flake tool lengths diverse $\left(\mathrm{F}_{(7,450)}=10.502, \mathrm{p}<0.001\right)$ by Ságvár being different from all assemblages except for Corvin-tér, Esztergom and Nadap. Out of these three the greatest similarity $(\mathrm{p}=1.000)$ was found with Corvint-tér (Tab. 206). Concerning the blade tools, also there are differences $\left(\mathrm{F}_{(7,576)}=8.216, \mathrm{p}<0.001\right)$, by Ságvár being different from all sites but Corvin-tér, and Corvin-tér being different from Bodrogkeresztúr, Nadap and Sajószentpéter (Tab. 207). Therefore, small sized domestic tools seem to correspond with EE assemblages.

Measuring backed bladelet lengths (Sajószentpéter excluded), ANOVA $\left(\mathrm{F}_{(6,231)}=\right.$ $3.275, \mathrm{p}=0.004)$ showed that some assemblages are different from the others, but the post hoc pairwise comparison did not realize this difference (Tab. 208). The widths of the backed bladelets are also different $\left(\mathrm{F}_{(6,231)}=31.299, \mathrm{p}<0.001\right)$, but in this case the post hoc test pointed out Esztergom specimens wider than the others, except for Hidasnémeti (Tab. 209). The mean thicknesses of the backed bladelets are also different $\left(\mathrm{F}_{(6,231)}=10.740, \mathrm{p}<0.001\right)$, which is due to that Esztergom and Hidasnémeti specimens are similarly thick and thicker than the others (Tab. 210). Other armatures, like trapeze-rectangles are also not different by length $\left(\mathrm{F}_{(2,4)}=0.810, \mathrm{p}=0.506\right)$, width $\left(\mathrm{F}_{(2,4)}=4.309, \mathrm{p}=0.100\right)$ and thickness $\left(\mathrm{F}_{(2,4)}=4.370, \mathrm{p}=0.099\right)$. The complete point assemblage also does not show differences by length $\left(\mathrm{F}_{(5,71)}=0.311, \mathrm{p}=0.905\right)$, width $\left(\mathrm{F}_{(5,71)}=1.174, \mathrm{p}=0.330\right)$ and thickness $\left(\mathrm{F}_{(5,71)}=0.482, \mathrm{p}=0.788\right)$. By sub-types of points, only the Gravette point widths were found different $\left(\mathrm{F}_{(3,10)}=6.541, \mathrm{p}=0.010\right)$ between Arka and Hidasnémeti $(\mathrm{p}=0.009)$, latter assemblage having wider specimens. 
Table 191. Blade lengths comparison between assemblages (complete specimens) with ANOVA and Tukey post hoc test

ANOVA

Length $[\mathrm{mm}]$

\begin{tabular}{|l|c|c|c|c|c|}
\hline & Sum of squares & df & Mean square & F & Sig. \\
\hline Between groups & 71142.930 & 7 & 10163.276 & 30.003 & 0.000 \\
\hline Within groups & 384131.315 & 1134 & 338.740 & & \\
\hline Total & 455274.245 & 1141 & & & \\
\hline
\end{tabular}

Multiple Comparisons

Dependent variable: length [mm]

Tukey HSD

\begin{tabular}{|c|c|c|c|c|c|c|}
\hline \multirow[b]{2}{*}{ (I) assemblage } & \multirow[b]{2}{*}{ (J) assemblage } & \multirow{2}{*}{$\begin{array}{c}\text { Mean } \\
\text { difference } \\
\text { (I-J) }\end{array}$} & \multirow[b]{2}{*}{ Std. error } & \multirow[b]{2}{*}{ Sig. } & \multicolumn{2}{|c|}{$95 \%$ Confidence interval } \\
\hline & & & & & $\begin{array}{l}\text { Lower } \\
\text { bound }\end{array}$ & $\begin{array}{l}\text { Upper } \\
\text { bound }\end{array}$ \\
\hline \multirow[t]{7}{*}{ Arka } & Bodrogkeresztúr & 2.27284 & 1.46958 & 0.782 & -2.1896 & 6.7353 \\
\hline & Corvin-tér & 22.32312 & 3.18525 & 0.000 & 12.6509 & 31.9954 \\
\hline & Esztergom & 4.21446 & 3.77968 & 0.954 & -7.2628 & 15.6917 \\
\hline & Hidasnémeti & -7.80060 & 2.17292 & 0.008 & -14.3988 & -1.2024 \\
\hline & Nadap & 5.28937 & 2.06517 & 0.171 & -0.9817 & 11.5604 \\
\hline & Ságvár & 18.84374 & 1.62794 & 0.000 & 13.9004 & 23.7871 \\
\hline & Sajószentpéter & 3.41686 & 3.77968 & 0.986 & -8.0604 & 14.8941 \\
\hline \multirow[t]{7}{*}{ Bodrogkeresztúr } & Arka & -2.27284 & 1.46958 & 0.782 & -6.7353 & 2.1896 \\
\hline & Corvin-tér & 20.05028 & 3.29131 & 0.000 & 10.0560 & 30.0446 \\
\hline & Esztergom & 1.94161 & 3.86948 & 1.000 & -9.8083 & 13.6916 \\
\hline & Hidasnémeti & -10.07345 & 2.32561 & 0.000 & -17.1353 & -3.0116 \\
\hline & Nadap & 3.01653 & 2.22527 & 0.877 & -3.7407 & 9.7737 \\
\hline & Ságvár & 16.57090 & 1.82676 & 0.000 & 11.0238 & 22.1180 \\
\hline & Sajószentpéter & 1.14401 & 3.86948 & 1.000 & -10.6059 & 12.8940 \\
\hline \multirow[t]{7}{*}{ Corvin-tér } & Arka & -22.32312 & 3.18525 & 0.000 & -31.9954 & -12.6509 \\
\hline & Bodrogkeresztúr & -20.05028 & 3.29131 & 0.000 & -30.0446 & -10.0560 \\
\hline & Esztergom & -18.10867 & 4.79156 & 0.004 & -32.6586 & -3.5587 \\
\hline & Hidasnémeti & -30.12373 & 3.65987 & 0.000 & -41.2372 & -19.0103 \\
\hline & Nadap & -17.03375 & 3.59694 & 0.000 & -27.9561 & -6.1114 \\
\hline & Ságvár & -3.47938 & 3.36500 & 0.969 & -13.6975 & 6.7387 \\
\hline & Sajószentpéter & -18.90627 & 4.79156 & 0.002 & -33.4562 & -4.3563 \\
\hline \multirow[t]{7}{*}{ Esztergom } & Arka & -4.21446 & 3.77968 & 0.954 & -15.6917 & 7.2628 \\
\hline & Bodrogkeresztúr & -1.94161 & 3.86948 & 1.000 & -13.6916 & 9.8083 \\
\hline & Corvin-tér & 18.10867 & 4.79156 & 0.004 & 3.5587 & 32.6586 \\
\hline & Hidasnémeti & -12.01506 & 4.18746 & 0.080 & -24.7306 & 0.7005 \\
\hline & Nadap & 1.07492 & 4.13257 & 1.000 & -11.4739 & 13.6238 \\
\hline & Ságvár & 14.62929 & 3.93235 & 0.005 & 2.6884 & 26.5702 \\
\hline & Sajószentpéter & -0.79760 & 5.20569 & 1.000 & -16.6051 & 15.0099 \\
\hline \multirow[t]{7}{*}{ Hidasnémeti } & Arka & 7.80060 & 2.17292 & 0.008 & 1.2024 & 14.3988 \\
\hline & Bodrogkeresztúr & 10.07345 & 2.32561 & 0.000 & 3.0116 & 17.1353 \\
\hline & Corvin-tér & 30.12373 & 3.65987 & 0.000 & 19.0103 & 41.2372 \\
\hline & Esztergom & 12.01506 & 4.18746 & 0.080 & -0.7005 & 24.7306 \\
\hline & Nadap & 13.08998 & 2.74112 & 0.000 & 4.7664 & 21.4136 \\
\hline & Ságvár & 26.64435 & 2.42878 & 0.000 & 19.2692 & 34.0195 \\
\hline & Sajószentpéter & 11.21746 & 4.18746 & 0.130 & -1.4981 & 23.9330 \\
\hline
\end{tabular}


Table 191. Continued

Multiple Comparisons

\begin{tabular}{|c|c|c|c|c|c|c|}
\hline \multicolumn{7}{|c|}{ Dependent variable: length [mm] } \\
\hline \multicolumn{7}{|l|}{ Tukey HSD } \\
\hline \multirow[b]{2}{*}{ (I) assemblage } & \multirow[b]{2}{*}{ (J) assemblage } & \multirow{2}{*}{$\begin{array}{c}\text { Mean } \\
\text { difference } \\
(\mathrm{I}-\mathrm{J})\end{array}$} & \multirow[b]{2}{*}{ Std. error } & \multirow[b]{2}{*}{ Sig. } & \multicolumn{2}{|c|}{ 95\% Confidence interval } \\
\hline & & & & & $\begin{array}{l}\text { Lower } \\
\text { bound }\end{array}$ & $\begin{array}{l}\text { Upper } \\
\text { bound }\end{array}$ \\
\hline \multirow[t]{7}{*}{ Nadap } & Arka & -5.28937 & 2.06517 & 0.171 & -11.5604 & 0.9817 \\
\hline & Bodrogkeresztúr & -3.01653 & 2.22527 & 0.877 & -9.7737 & 3.7407 \\
\hline & Corvin-tér & 17.03375 & 3.59694 & 0.000 & 6.1114 & 27.9561 \\
\hline & Esztergom & -1.07492 & 4.13257 & 1.000 & -13.6238 & 11.4739 \\
\hline & Hidasnémeti & -13.08998 & 2.74112 & 0.000 & -21.4136 & -4.7664 \\
\hline & Ságvár & 13.55437 & 2.33288 & 0.000 & 6.4704 & 20.6383 \\
\hline & Sajószentpéter & -1.87252 & 4.13257 & 1.000 & -14.4214 & 10.6763 \\
\hline \multirow[t]{7}{*}{ Ságvár } & Arka & -18.84374 & 1.62794 & 0.000 & -23.7871 & -13.9004 \\
\hline & Bodrogkeresztúr & -16.57090 & 1.82676 & 0.000 & -22.1180 & -11.0238 \\
\hline & Corvin-tér & 3.47938 & 3.36500 & 0.969 & -6.7387 & 13.6975 \\
\hline & Esztergom & -14.62929 & 3.93235 & 0.005 & -26.5702 & -2.6884 \\
\hline & Hidasnémeti & -26.64435 & 2.42878 & 0.000 & -34.0195 & -19.2692 \\
\hline & Nadap & -13.55437 & 2.33288 & 0.000 & -20.6383 & -6.4704 \\
\hline & Sajószentpéter & -15.42689 & 3.93235 & 0.002 & -27.3678 & -3.4860 \\
\hline \multirow[t]{7}{*}{ Sajószentpéter } & Arka & -3.41686 & 3.77968 & 0.986 & -14.8941 & 8.0604 \\
\hline & Bodrogkeresztúr & -1.14401 & 3.86948 & 1.000 & -12.8940 & 10.6059 \\
\hline & Corvin-tér & 18.90627 & 4.79156 & 0.002 & 4.3563 & 33.4562 \\
\hline & Esztergom & 0.79760 & 5.20569 & 1.000 & -15.0099 & 16.6051 \\
\hline & Hidasnémeti & -11.21746 & 4.18746 & 0.130 & -23.9330 & 1.4981 \\
\hline & Nadap & 1.87252 & 4.13257 & 1.000 & -10.6763 & 14.4214 \\
\hline & Ságvár & 15.42689 & 3.93235 & 0.002 & 3.4860 & 27.3678 \\
\hline
\end{tabular}

Table 192. Blade lengths comparison between periods (complete specimens) with ANOVA and Tukey post hoc test

ANOVA

\begin{tabular}{|l|c|c|c|c|c|}
\hline Length [mm] & Sum of squares & df & Mean square & F & Sig. \\
\hline & 64065.983 & 2 & 32032.992 & 93.264 & 0.000 \\
\hline Between groups & 391208.262 & 1139 & 343.466 & & \\
\hline Within groups & 455274.245 & 1141 & & & \\
\hline Total &
\end{tabular}

Multiple Comparisons

\begin{tabular}{|l|l|c|c|c|c|c|}
\hline \multicolumn{6}{|l|}{ Dependent variable: length [mm] } \\
\hline \multicolumn{3}{|l|}{ Tukey HSD } \\
\hline \multirow{3}{*}{$(\mathrm{I})$ age } & $(\mathrm{J})$ age & $\begin{array}{c}\text { Mean } \\
\text { difference } \\
(\mathrm{I}-\mathrm{J})\end{array}$ & Std. error & Sig. & $\begin{array}{c}\text { Lower } \\
\text { bound }\end{array}$ & $\begin{array}{c}\text { Upper } \\
\text { bound }\end{array}$ \\
\hline \multirow{2}{*}{ LG } & EEG & 19.47722 & 1.42745 & 0.000 & 16.1273 & 22.8271 \\
\cline { 2 - 7 } & LEG & 5.11270 & 1.80656 & 0.013 & 0.8731 & 9.3523 \\
\hline \multirow{2}{*}{ EEG } & LG & -19.47722 & 1.42745 & 0.000 & -22.8271 & -16.1273 \\
\cline { 2 - 7 } & LEG & -14.36453 & 2.10976 & 0.000 & -19.3157 & -9.4134 \\
\hline \multirow{2}{*}{ LEG } & LG & -5.11270 & 1.80656 & 0.013 & -9.3523 & -0.8731 \\
\cline { 2 - 7 } & EEG & 14.36453 & 2.10976 & 0.000 & 9.4134 & 19.3157 \\
\hline
\end{tabular}


Table 193. Local blade lengths comparison within LG (complete specimens) with ANOVA and Tukey post hoc test

ANOVA

\begin{tabular}{|l|c|r|c|c|c|}
\hline Length [mm] & Sum of squares & df & Mean square & F & Sig. \\
\hline Between groups & 17624.140 & 3 & 5874.713 & 18.538 & 0.000 \\
\hline Within groups & 69716.801 & 220 & 316.895 & & \\
\hline Total & 87340.941 & 223 & & & \\
\hline
\end{tabular}

Multiple Comparisons

Dependent variable: length [mm]

Tukey HSD

\begin{tabular}{|c|c|c|c|c|c|c|}
\hline \multirow[b]{2}{*}{ (I) assemblage } & \multirow[b]{2}{*}{ (J) assemblage } & \multirow{2}{*}{$\begin{array}{c}\text { Mean } \\
\text { difference } \\
(\mathrm{I}-\mathrm{J})\end{array}$} & \multirow[b]{2}{*}{ Std. error } & \multirow[b]{2}{*}{ Sig. } & \multicolumn{2}{|c|}{$95 \%$ Confidence interval } \\
\hline & & & & & $\begin{array}{l}\text { Lower } \\
\text { bound }\end{array}$ & $\begin{array}{l}\text { Upper } \\
\text { bound }\end{array}$ \\
\hline \multirow[t]{3}{*}{ Arka } & Bodrogkeresztúr & -3.38995 & 4.15456 & 0.847 & -14.1452 & 7.3653 \\
\hline & Hidasnémeti & -19.59266 & 2.68524 & 0.000 & -26.5442 & -12.6412 \\
\hline & Sajószentpéter & -8.37520 & 4.01972 & 0.162 & -18.7814 & 2.0309 \\
\hline \multirow[t]{3}{*}{ Bodrogkeresztúr } & Arka & 3.38995 & 4.15456 & 0.847 & -7.3653 & 14.1452 \\
\hline & Hidasnémeti & -16.20271 & 4.18404 & 0.001 & -27.0342 & -5.3712 \\
\hline & Sajószentpéter & -4.98525 & 5.14333 & 0.767 & -18.3002 & 8.3297 \\
\hline \multirow[t]{3}{*}{ Hidasnémeti } & Arka & 19.59266 & 2.68524 & 0.000 & 12.6412 & 26.5442 \\
\hline & Bodrogkeresztúr & 16.20271 & 4.18404 & 0.001 & 5.3712 & 27.0342 \\
\hline & Sajószentpéter & 11.21746 & 4.05018 & 0.031 & 0.7325 & 21.7025 \\
\hline \multirow[t]{3}{*}{ Sajószentpéter } & Arka & 8.37520 & 4.01972 & 0.162 & -2.0309 & 18.7814 \\
\hline & Bodrogkeresztúr & 4.98525 & 5.14333 & 0.767 & -8.3297 & 18.3002 \\
\hline & Hidasnémeti & -11.21746 & 4.05018 & 0.031 & -21.7025 & -0.7325 \\
\hline
\end{tabular}


Table 194. Regional blade lengths comparison between assemblages (complete specimens) with ANOVA and Tukey post hoc test

ANOVA

\begin{tabular}{|l|c|r|c|c|c|}
\hline Length [mm] & Mean square & F & Sig. \\
\hline Between groups & Sum of squares & df & Meare & 0.000 \\
\hline Within groups & 95433.898 & 7 & 7019.128 & 31.115 & \\
\hline Total & 144556.766 & 423 & 225.586 & & \\
\hline
\end{tabular}

Multiple Comparisons

\begin{tabular}{|c|c|c|c|c|c|c|}
\hline \multicolumn{7}{|c|}{ Dependent variable: length [mm] } \\
\hline \multicolumn{7}{|l|}{ Tukey HSD } \\
\hline \multirow[b]{2}{*}{ (I) assemblage } & \multirow[b]{2}{*}{$(\mathrm{J})$ assemblage } & \multirow{2}{*}{$\begin{array}{c}\text { Mean } \\
\text { difference } \\
(\mathrm{I}-\mathrm{J})\end{array}$} & \multirow[b]{2}{*}{ Std. error } & \multirow[b]{2}{*}{ Sig. } & \multicolumn{2}{|c|}{ 95\% Confidence interval } \\
\hline & & & & & $\begin{array}{l}\text { Lower } \\
\text { bound }\end{array}$ & $\begin{array}{l}\text { Upper } \\
\text { bound }\end{array}$ \\
\hline \multirow[t]{7}{*}{ Arka } & Bodrogkeresztúr & -3.38995 & 3.50529 & 0.979 & -14.0677 & 7.2877 \\
\hline & Corvin-tér & 10.53106 & 2.95724 & 0.010 & 1.5228 & 19.5393 \\
\hline & Esztergom & 5.21440 & 10.73648 & 1.000 & -27.4907 & 37.9195 \\
\hline & Hidasnémeti & -19.59266 & 2.26559 & 0.000 & -26.4940 & -12.6913 \\
\hline & Nadap & -7.84960 & 3.39152 & 0.288 & -18.1807 & 2.4815 \\
\hline & Ságvár & 8.19773 & 2.01135 & 0.001 & 2.0708 & 14.3246 \\
\hline & Sajószentpéter & -8.37520 & 3.39152 & 0.212 & -18.7063 & 1.9559 \\
\hline \multirow{7}{*}{ Bodrogkeresztúr } & Arka & 3.38995 & 3.50529 & 0.979 & -7.2877 & 14.0677 \\
\hline & Corvin-tér & 13.92101 & 4.00928 & 0.013 & 1.7081 & 26.1340 \\
\hline & Esztergom & 8.60435 & 11.07254 & 0.994 & -25.1245 & 42.3332 \\
\hline & Hidasnémeti & -16.20271 & 3.53016 & 0.000 & -26.9562 & -5.4492 \\
\hline & Nadap & -4.45965 & 4.33953 & 0.970 & -17.6786 & 8.7593 \\
\hline & Ságvár & 11.58768 & 3.37263 & 0.015 & 1.3141 & 21.8613 \\
\hline & Sajószentpéter & -4.98525 & 4.33953 & 0.945 & -18.2042 & 8.2337 \\
\hline \multirow{7}{*}{ Corvin-tér } & Arka & -10.53106 & 2.95724 & 0.010 & -19.5393 & -1.5228 \\
\hline & Bodrogkeresztúr & -13.92101 & 4.00928 & 0.013 & -26.1340 & -1.7081 \\
\hline & \begin{tabular}{|l} 
Esztergom \\
\end{tabular} & -5.31667 & 10.91143 & 1.000 & -38.5547 & 27.9214 \\
\hline & Hidasnémeti & -30.12373 & 2.98668 & 0.000 & -39.2216 & -21.0258 \\
\hline & Nadap & -18.38067 & 3.91021 & 0.000 & -30.2918 & -6.4695 \\
\hline & Ságvár & -2.33333 & 2.79872 & 0.991 & -10.8587 & 6.1920 \\
\hline & Sajószentpéter & -18.90627 & 3.91021 & 0.000 & -30.8174 & -6.9951 \\
\hline \multirow{7}{*}{ Esztergom } & Arka & -5.21440 & 10.73648 & 1.000 & -37.9195 & 27.4907 \\
\hline & Bodrogkeresztúr & -8.60435 & 11.07254 & 0.994 & -42.3332 & 25.1245 \\
\hline & Corvin-tér & 5.31667 & 10.91143 & 1.000 & -27.9214 & 38.5547 \\
\hline & \begin{tabular}{|l|} 
Hidasnémeti \\
\end{tabular} & -24.80706 & 10.74462 & 0.291 & -57.5370 & 7.9229 \\
\hline & Nadap & -13.06400 & 11.03705 & 0.936 & -46.6847 & 20.5567 \\
\hline & Ságvár & 2.98333 & 10.69390 & 1.000 & -29.5921 & 35.5588 \\
\hline & Sajószentpéter & -13.58960 & 11.03705 & 0.922 & -47.2103 & 20.0311 \\
\hline \multirow{7}{*}{ Hidasnémeti } & Arka & 19.59266 & 2.26559 & 0.000 & 12.6913 & 26.4940 \\
\hline & Bodrogkeresztúr & 16.20271 & 3.53016 & 0.000 & 5.4492 & 26.9562 \\
\hline & Corvin-tér & 30.12373 & 2.98668 & 0.000 & 21.0258 & 39.2216 \\
\hline & Esztergom & 24.80706 & 10.74462 & 0.291 & -7.9229 & 57.5370 \\
\hline & Nadap & 11.74306 & 3.41722 & 0.015 & 1.3336 & 22.1525 \\
\hline & Ságvár & 27.79039 & 2.05439 & 0.000 & 21.5324 & 34.0484 \\
\hline & Sajószentpéter & 11.21746 & 3.41722 & 0.024 & 0.8080 & 21.6269 \\
\hline
\end{tabular}


Table 194. Continued

Multiple Comparisons

\begin{tabular}{|c|c|c|c|c|c|c|}
\hline \multicolumn{7}{|c|}{ Dependent variable: length [mm] } \\
\hline \multicolumn{7}{|l|}{ Tukey HSD } \\
\hline \multirow[b]{2}{*}{ (I) assemblage } & \multirow[b]{2}{*}{$(\mathrm{J})$ assemblage } & \multirow{2}{*}{$\begin{array}{c}\text { Mean } \\
\text { difference } \\
(\mathrm{I}-\mathrm{J})\end{array}$} & \multirow[b]{2}{*}{ Std. error } & \multirow[b]{2}{*}{ Sig. } & \multicolumn{2}{|c|}{ 95\% Confidence interval } \\
\hline & & & & & $\begin{array}{l}\text { Lower } \\
\text { bound }\end{array}$ & $\begin{array}{l}\text { Upper } \\
\text { bound }\end{array}$ \\
\hline \multirow[t]{7}{*}{ Nadap } & Arka & 7.84960 & 3.39152 & 0.288 & -2.4815 & 18.1807 \\
\hline & Bodrogkeresztúr & 4.45965 & 4.33953 & 0.970 & -8.7593 & 17.6786 \\
\hline & Corvin-tér & 18.38067 & 3.91021 & 0.000 & 6.4695 & 30.2918 \\
\hline & Esztergom & 13.06400 & 11.03705 & 0.936 & -20.5567 & 46.6847 \\
\hline & Hidasnémeti & -11.74306 & 3.41722 & 0.015 & -22.1525 & -1.3336 \\
\hline & Ságvár & 16.04733 & 3.25423 & 0.000 & 6.1344 & 25.9603 \\
\hline & Sajószentpéter & -0.52560 & 4.24816 & 1.000 & -13.4662 & 12.4150 \\
\hline \multirow[t]{7}{*}{ Ságvár } & Arka & -8.19773 & 2.01135 & 0.001 & -14.3246 & -2.0708 \\
\hline & Bodrogkeresztúr & -11.58768 & 3.37263 & 0.015 & -21.8613 & -1.3141 \\
\hline & Corvin-tér & 2.33333 & 2.79872 & 0.991 & -6.1920 & 10.8587 \\
\hline & Esztergom & -2.98333 & 10.69390 & 1.000 & -35.5588 & 29.5921 \\
\hline & Hidasnémeti & -27.79039 & 2.05439 & 0.000 & -34.0484 & -21.5324 \\
\hline & Nadap & -16.04733 & 3.25423 & 0.000 & -25.9603 & -6.1344 \\
\hline & Sajószentpéter & -16.57293 & 3.25423 & 0.000 & -26.4859 & -6.6600 \\
\hline \multirow[t]{7}{*}{ Sajószentpéter } & Arka & 8.37520 & 3.39152 & 0.212 & -1.9559 & 18.7063 \\
\hline & Bodrogkeresztúr & 4.98525 & 4.33953 & 0.945 & -8.2337 & 18.2042 \\
\hline & Corvin-tér & 18.90627 & 3.91021 & 0.000 & 6.9951 & 30.8174 \\
\hline & Esztergom & 13.58960 & 11.03705 & 0.922 & -20.0311 & 47.2103 \\
\hline & Hidasnémeti & -11.21746 & 3.41722 & 0.024 & -21.6269 & -0.8080 \\
\hline & Nadap & 0.52560 & 4.24816 & 1.000 & -12.4150 & 13.4662 \\
\hline & Ságvár & 16.57293 & 3.25423 & 0.000 & 6.6600 & 26.4859 \\
\hline
\end{tabular}

Table 195. Blade thicknesses comparison between assemblages (complete specimens) with ANOVA and Tukey post hoc test

ANOVA

\begin{tabular}{|l|c|c|c|c|c|}
\hline Thickness [mm] & Sum of squares & df & Mean square & F & Sig. \\
\hline Between groups & 557.923 & 7 & 79.703 & 5.644 & 0.000 \\
\hline Within groups & 16015.339 & 1134 & 14.123 & & \\
\hline Total & 16573.263 & 1141 & & & \\
\hline
\end{tabular}

Multiple Comparisons

Dependent variable: thickness [mm] Tukey HSD

\begin{tabular}{|c|c|c|c|c|c|c|}
\hline \multirow[b]{2}{*}{ (I) assemblage } & \multirow[b]{2}{*}{$(\mathrm{J})$ assemblage } & \multirow{2}{*}{$\begin{array}{c}\text { Mean } \\
\text { difference } \\
(\mathrm{I}-\mathrm{J})\end{array}$} & \multirow[b]{2}{*}{ Std. error } & \multirow[b]{2}{*}{ Sig. } & \multicolumn{2}{|c|}{ 95\% Confidence interval } \\
\hline & & & & & $\begin{array}{l}\text { Lower } \\
\text { bound }\end{array}$ & $\begin{array}{l}\text { Upper } \\
\text { bound }\end{array}$ \\
\hline \multirow[t]{7}{*}{ Arka } & Bodrogkeresztúr & 0.60819 & 0.30007 & 0.464 & -0.3030 & 1.5194 \\
\hline & Corvin-tér & 2.24107 & 0.65039 & 0.014 & 0.2661 & 4.2160 \\
\hline & Esztergom & 1.47763 & 0.77176 & 0.541 & -0.8659 & 3.8211 \\
\hline & Hidasnémeti & 0.32128 & 0.44368 & 0.996 & -1.0260 & 1.6685 \\
\hline & Nadap & 1.76632 & 0.42168 & 0.001 & 0.4859 & 3.0468 \\
\hline & Ságvár & 1.49282 & 0.33240 & 0.000 & 0.4834 & 2.5022 \\
\hline & Sajószentpéter & 0.52683 & 0.77176 & 0.997 & -1.8167 & 2.8703 \\
\hline
\end{tabular}


Table 195. Continued

Multiple Comparisons

\begin{tabular}{|c|c|c|c|c|c|c|}
\hline \multirow{2}{*}{\multicolumn{7}{|c|}{$\begin{array}{l}\text { Dependent variable: thickness [mm] } \\
\text { Tukey HSD }\end{array}$}} \\
\hline & & & & & & \\
\hline \multirow[b]{2}{*}{ (I) assemblage } & \multirow[b]{2}{*}{$(\mathrm{J})$ assemblage } & \multirow{2}{*}{$\begin{array}{c}\text { Mean } \\
\text { difference } \\
(\mathrm{I}-\mathrm{J})\end{array}$} & \multirow[b]{2}{*}{ Std. error } & \multirow[b]{2}{*}{ Sig. } & \multicolumn{2}{|c|}{ 95\% Confidence interval } \\
\hline & & & & & $\begin{array}{l}\text { Lower } \\
\text { bound }\end{array}$ & $\begin{array}{l}\text { Upper } \\
\text { bound }\end{array}$ \\
\hline \multirow[t]{7}{*}{ Bodrogkeresztúr } & Arka & -0.60819 & 0.30007 & 0.464 & -1.5194 & 0.3030 \\
\hline & Corvin-tér & 1.63289 & 0.67204 & 0.228 & -0.4078 & 3.6736 \\
\hline & Esztergom & 0.86945 & 0.79010 & 0.957 & -1.5297 & 3.2686 \\
\hline & Hidasnémeti & -0.28691 & 0.47486 & 0.999 & -1.7289 & 1.1550 \\
\hline & Nadap & 1.15813 & 0.45437 & 0.176 & -0.2216 & 2.5379 \\
\hline & Ságvár & 0.88463 & 0.37300 & 0.256 & -0.2480 & 2.0173 \\
\hline & Sajószentpéter & -0.08135 & 0.79010 & 1.000 & -2.4805 & 2.3178 \\
\hline \multirow[t]{7}{*}{ Corvin-tér } & Arka & -2.24107 & 0.65039 & 0.014 & -4.2160 & -0.2661 \\
\hline & Bodrogkeresztúr & -1.63289 & 0.67204 & 0.228 & -3.6736 & 0.4078 \\
\hline & Esztergom & -0.76344 & 0.97837 & 0.994 & -3.7344 & 2.2075 \\
\hline & Hidasnémeti & -1.91980 & 0.74730 & 0.168 & -4.1890 & 0.3494 \\
\hline & Nadap & -0.47476 & 0.73445 & 0.998 & -2.7050 & 1.7555 \\
\hline & Ságvár & -0.74826 & 0.68709 & 0.959 & -2.8347 & 1.3381 \\
\hline & Sajószentpéter & -1.71424 & 0.97837 & 0.653 & -4.6852 & 1.2567 \\
\hline \multirow[t]{7}{*}{ Esztergom } & Arka & -1.47763 & 0.77176 & 0.541 & -3.8211 & 0.8659 \\
\hline & Bodrogkeresztúr & -0.86945 & 0.79010 & 0.957 & -3.2686 & 1.5297 \\
\hline & Corvin-tér & 0.76344 & 0.97837 & 0.994 & -2.2075 & 3.7344 \\
\hline & Hidasnémeti & -1.15635 & 0.85502 & 0.878 & -3.7527 & 1.4400 \\
\hline & Nadap & 0.28869 & 0.84382 & 1.000 & -2.2736 & 2.8510 \\
\hline & Ságvár & 0.01519 & 0.80294 & 1.000 & -2.4230 & 2.4534 \\
\hline & Sajószentpéter & -0.95080 & 1.06293 & 0.987 & -4.1785 & 2.2769 \\
\hline \multirow{7}{*}{ Hidasnémeti } & Arka & -0.32128 & 0.44368 & 0.996 & -1.6685 & 1.0260 \\
\hline & Bodrogkeresztúr & 0.28691 & 0.47486 & 0.999 & -1.1550 & 1.7289 \\
\hline & Corvin-tér & 1.91980 & 0.74730 & 0.168 & -0.3494 & 4.1890 \\
\hline & Esztergom & 1.15635 & 0.85502 & 0.878 & -1.4400 & 3.7527 \\
\hline & Nadap & 1.44504 & 0.55970 & 0.164 & -0.2545 & 3.1446 \\
\hline & Ságvár & 1.17154 & 0.49593 & 0.261 & -0.3344 & 2.6775 \\
\hline & Sajószentpéter & 0.20555 & 0.85502 & 1.000 & -2.3908 & 2.8019 \\
\hline \multirow[t]{7}{*}{ Nadap } & Arka & -1.76632 & 0.42168 & 0.001 & -3.0468 & -0.4859 \\
\hline & Bodrogkeresztúr & -1.15813 & 0.45437 & 0.176 & -2.5379 & 0.2216 \\
\hline & Corvin-tér & 0.47476 & 0.73445 & 0.998 & -1.7555 & 2.7050 \\
\hline & Esztergom & -0.28869 & 0.84382 & 1.000 & -2.8510 & 2.2736 \\
\hline & Hidasnémeti & -1.44504 & 0.55970 & 0.164 & -3.1446 & 0.2545 \\
\hline & Ságvár & -0.27350 & 0.47634 & 0.999 & -1.7200 & 1.1730 \\
\hline & Sajószentpéter & -1.23949 & 0.84382 & 0.824 & -3.8018 & 1.3228 \\
\hline \multirow[t]{7}{*}{ Ságvár } & Arka & -1.49282 & 0.33240 & 0.000 & -2.5022 & -0.4834 \\
\hline & Bodrogkeresztúr & -0.88463 & 0.37300 & 0.256 & -2.0173 & 0.2480 \\
\hline & Corvin-tér & 0.74826 & 0.68709 & 0.959 & -1.3381 & 2.8347 \\
\hline & Esztergom & -0.01519 & 0.80294 & 1.000 & -2.4534 & 2.4230 \\
\hline & Hidasnémeti & -1.17154 & 0.49593 & 0.261 & -2.6775 & 0.3344 \\
\hline & Nadap & 0.27350 & 0.47634 & 0.999 & -1.1730 & 1.7200 \\
\hline & Sajószentpéter & -0.96599 & 0.80294 & 0.931 & -3.4042 & 1.4722 \\
\hline \multirow{7}{*}{ Sajószentpéter } & Arka & -0.52683 & 0.77176 & 0.997 & -2.8703 & 1.8167 \\
\hline & Bodrogkeresztúr & 0.08135 & 0.79010 & 1.000 & -2.3178 & 2.4805 \\
\hline & Corvin-tér & 1.71424 & 0.97837 & 0.653 & -1.2567 & 4.6852 \\
\hline & Esztergom & 0.95080 & 1.06293 & 0.987 & -2.2769 & 4.1785 \\
\hline & Hidasnémeti & -0.20555 & 0.85502 & 1.000 & -2.8019 & 2.3908 \\
\hline & Nadap & 1.23949 & 0.84382 & 0.824 & -1.3228 & 3.8018 \\
\hline & Ságvár & 0.96599 & 0.80294 & 0.931 & -1.4722 & 3.4042 \\
\hline
\end{tabular}


Table 196. Regional blade thicknesses comparison between assemblages (complete specimens) with ANOVA and Tukey post hoc test

ANOVA

\begin{tabular}{|l|c|r|c|c|c|}
\hline \multicolumn{7}{|l|}{ Thickness [mm] } & Sum of squares & df & Mean square & F & Sig. \\
\hline Between groups & 197.737 & 7 & 28.248 & 3.283 & 0.002 \\
\hline Within groups & 3639.117 & 423 & 8.603 & & \\
\hline Total & 3836.854 & 430 & & & \\
\hline
\end{tabular}

Multiple Comparisons

\begin{tabular}{|c|c|c|c|c|c|c|}
\hline \multicolumn{7}{|c|}{ Dependent variable: thickness [mm] } \\
\hline \multicolumn{7}{|c|}{ Tukey HSD } \\
\hline \multirow[b]{2}{*}{ (I) assemblage } & \multirow[b]{2}{*}{ (J) assemblage } & \multirow{2}{*}{$\begin{array}{c}\text { Mean } \\
\text { difference } \\
(\mathrm{I}-\mathrm{J})\end{array}$} & \multirow[b]{2}{*}{ Std. error } & \multirow[b]{2}{*}{ Sig. } & \multicolumn{2}{|c|}{ 95\% Confidence interval } \\
\hline & & & & & $\begin{array}{l}\text { Lower } \\
\text { bound }\end{array}$ & $\begin{array}{l}\text { Upper } \\
\text { bound }\end{array}$ \\
\hline \multirow[t]{7}{*}{ Arka } & Bodrogkeresztúr & -0.36378 & 0.68453 & 0.999 & -2.4490 & 1.7214 \\
\hline & Corvin-tér & 0.45131 & 0.57751 & 0.994 & -1.3079 & 2.2105 \\
\hline & Esztergom & 1.03187 & 2.09669 & 1.000 & -5.3550 & 7.4187 \\
\hline & Hidasnémeti & -1.46848 & 0.44244 & 0.022 & -2.8162 & -0.1207 \\
\hline & Nadap & 0.33187 & 0.66232 & 1.000 & -1.6857 & 2.3494 \\
\hline & Ságvár & 0.03742 & 0.39279 & 1.000 & -1.1591 & 1.2339 \\
\hline & Sajószentpéter & -1.26293 & 0.66232 & 0.547 & -3.2805 & 0.7546 \\
\hline \multirow[t]{7}{*}{ Bodrogkeresztúr } & Arka & 0.36378 & 0.68453 & 0.999 & -1.7214 & 2.4490 \\
\hline & Corvin-tér & 0.81510 & 0.78296 & 0.968 & -1.5699 & 3.2001 \\
\hline & Esztergom & 1.39565 & 2.16231 & 0.998 & -5.1911 & 7.9824 \\
\hline & Hidasnémeti & -1.10470 & 0.68939 & 0.749 & -3.2047 & 0.9953 \\
\hline & Nadap & 0.69565 & 0.84745 & 0.992 & -1.8858 & 3.2771 \\
\hline & Ságvár & 0.40121 & 0.65863 & 0.999 & -1.6051 & 2.4075 \\
\hline & Sajószentpéter & -0.89915 & 0.84745 & 0.964 & -3.4806 & 1.6823 \\
\hline \multirow[t]{7}{*}{ Corvin-tér } & Arka & -0.45131 & 0.57751 & 0.994 & -2.2105 & 1.3079 \\
\hline & Bodrogkeresztúr & -0.81510 & 0.78296 & 0.968 & -3.2001 & 1.5699 \\
\hline & Esztergom & 0.58056 & 2.13085 & 1.000 & -5.9104 & 7.0715 \\
\hline & Hidasnémeti & -1.91980 & 0.58326 & 0.024 & -3.6965 & -0.1431 \\
\hline & Nadap & -0.11944 & 0.76361 & 1.000 & -2.4455 & 2.2066 \\
\hline & Ságvár & -0.41389 & 0.54655 & 0.995 & -2.0788 & 1.2510 \\
\hline & Sajószentpéter & -1.71424 & 0.76361 & 0.327 & -4.0403 & 0.6118 \\
\hline \multirow[t]{7}{*}{ Esztergom } & Arka & -1.03187 & 2.09669 & 1.000 & -7.4187 & 5.3550 \\
\hline & Bodrogkeresztúr & -1.39565 & 2.16231 & 0.998 & -7.9824 & 5.1911 \\
\hline & Corvin-tér & -0.58056 & 2.13085 & 1.000 & -7.0715 & 5.9104 \\
\hline & Hidasnémeti & -2.50035 & 2.09828 & 0.934 & -8.8921 & 3.8914 \\
\hline & Nadap & -0.70000 & 2.15538 & 1.000 & -7.2657 & 5.8657 \\
\hline & Ságvár & -0.99444 & 2.08837 & 1.000 & -7.3560 & 5.3671 \\
\hline & Sajószentpéter & -2.29480 & 2.15538 & 0.964 & -8.8605 & 4.2709 \\
\hline \multirow[t]{7}{*}{ Hidasnémeti } & Arka & 1.46848 & 0.44244 & 0.022 & 0.1207 & 2.8162 \\
\hline & Bodrogkeresztúr & 1.10470 & 0.68939 & 0.749 & -0.9953 & 3.2047 \\
\hline & Corvin-tér & 1.91980 & 0.58326 & 0.024 & 0.1431 & 3.6965 \\
\hline & Esztergom & 2.50035 & 2.09828 & 0.934 & -3.8914 & 8.8921 \\
\hline & Nadap & 1.80035 & 0.66734 & 0.126 & -0.2325 & 3.8332 \\
\hline & Ságvár & 1.50591 & 0.40119 & 0.005 & 0.2838 & 2.7280 \\
\hline & Sajószentpéter & 0.20555 & 0.66734 & 1.000 & -1.8273 & 2.2384 \\
\hline
\end{tabular}


Table 196. Continued

\begin{tabular}{|c|c|c|c|c|c|c|}
\hline \multicolumn{7}{|c|}{ Dependent variable: thickness [mm] } \\
\hline \multirow[b]{3}{*}{ (I) assemblage } & \multirow[b]{3}{*}{ (J) assemblage } & & & & & \\
\hline & & \multirow{2}{*}{$\begin{array}{c}\text { Mean } \\
\text { difference } \\
(\mathrm{I}-\mathrm{J})\end{array}$} & \multirow[b]{2}{*}{ Std. error } & \multirow[b]{2}{*}{ Sig. } & \multicolumn{2}{|c|}{ 95\% Confidence interval } \\
\hline & & & & & $\begin{array}{l}\text { Lower } \\
\text { bound }\end{array}$ & $\begin{array}{l}\text { Upper } \\
\text { bound }\end{array}$ \\
\hline \multirow[t]{7}{*}{ Nadap } & Arka & -0.33187 & 0.66232 & 1.000 & -2.3494 & 1.6857 \\
\hline & Bodrogkeresztúr & -0.69565 & 0.84745 & 0.992 & -3.2771 & 1.8858 \\
\hline & Corvin-tér & 0.11944 & 0.76361 & 1.000 & -2.2066 & 2.4455 \\
\hline & Esztergom & 0.70000 & 2.15538 & 1.000 & -5.8657 & 7.2657 \\
\hline & Hidasnémeti & -1.80035 & 0.66734 & 0.126 & -3.8332 & 0.2325 \\
\hline & Ságvár & -0.29444 & 0.63551 & 1.000 & -2.2303 & 1.6414 \\
\hline & Sajószentpéter & -1.59480 & 0.82961 & 0.536 & -4.1219 & 0.9323 \\
\hline \multirow[t]{7}{*}{ Ságvár } & Arka & -0.03742 & 0.39279 & 1.000 & -1.2339 & 1.1591 \\
\hline & Bodrogkeresztúr & -0.40121 & 0.65863 & 0.999 & -2.4075 & 1.6051 \\
\hline & Corvin-tér & 0.41389 & 0.54655 & 0.995 & -1.2510 & 2.0788 \\
\hline & Esztergom & 0.99444 & 2.08837 & 1.000 & -5.3671 & 7.3560 \\
\hline & Hidasnémeti & -1.50591 & 0.40119 & 0.005 & -2.7280 & -0.2838 \\
\hline & Nadap & 0.29444 & 0.63551 & 1.000 & -1.6414 & 2.2303 \\
\hline & Sajószentpéter & -1.30036 & 0.63551 & 0.452 & -3.2362 & 0.6355 \\
\hline \multirow[t]{7}{*}{ Sajószentpéter } & Arka & 1.26293 & 0.66232 & 0.547 & -0.7546 & 3.2805 \\
\hline & Bodrogkeresztúr & 0.89915 & 0.84745 & 0.964 & -1.6823 & 3.4806 \\
\hline & Corvin-tér & 1.71424 & 0.76361 & 0.327 & -0.6118 & 4.0403 \\
\hline & Esztergom & 2.29480 & 2.15538 & 0.964 & -4.2709 & 8.8605 \\
\hline & Hidasnémeti & -0.20555 & 0.66734 & 1.000 & -2.2384 & 1.8273 \\
\hline & Nadap & 1.59480 & 0.82961 & 0.536 & -0.9323 & 4.1219 \\
\hline & Ságvár & 1.30036 & 0.63551 & 0.452 & -0.6355 & 3.2362 \\
\hline
\end{tabular}

Table 197. Transcarpathian blade thicknesses comparison between assemblages (complete specimens) with ANOVA and Tukey post hoc test

ANOVA

\begin{tabular}{|l|c|r|c|c|c|}
\hline Thickness [mm] & Sum of squares & df & Mean square & F & Sig. \\
\hline Between groups & 65.618 & 3 & 21.873 & 3.576 & 0.015 \\
\hline Within groups & 1174.299 & 192 & 6.116 & & \\
\hline Total & 1239.917 & 195 & & & \\
\hline
\end{tabular}

Multiple Comparisons

Dependent variable: thickness [mm]

Tukey HSD

\begin{tabular}{|c|c|c|c|c|c|c|}
\hline \multirow[b]{2}{*}{ (I) assemblage } & \multirow[b]{2}{*}{$(\mathrm{J})$ assemblage } & \multirow{2}{*}{$\begin{array}{c}\text { Mean } \\
\text { difference } \\
(\mathrm{I}-\mathrm{J})\end{array}$} & \multirow[b]{2}{*}{ Std. error } & \multirow[b]{2}{*}{ Sig. } & \multicolumn{2}{|c|}{$95 \%$ Confidence interval } \\
\hline & & & & & $\begin{array}{l}\text { Lower } \\
\text { bound }\end{array}$ & $\begin{array}{l}\text { Upper } \\
\text { bound }\end{array}$ \\
\hline \multirow[t]{3}{*}{ Arka } & Bodrogkeresztúr & 0.53239 & 0.54771 & 0.766 & -0.8871 & 1.9519 \\
\hline & Esztergom & -1.09001 & 0.59963 & 0.268 & -2.6441 & 0.4640 \\
\hline & Nadap & -0.91589 & 0.40950 & 0.117 & -1.9772 & 0.1454 \\
\hline \multirow[t]{3}{*}{ Bodrogkeresztúr } & Arka & -0.53239 & 0.54771 & 0.766 & -1.9519 & 0.8871 \\
\hline & Esztergom & -1.62240 & 0.70458 & 0.101 & -3.4485 & 0.2037 \\
\hline & Nadap & -1.44828 & 0.55188 & 0.046 & -2.8786 & -0.0180 \\
\hline
\end{tabular}


Table 197. Continued

Multiple Comparisons

\begin{tabular}{|c|c|c|c|c|c|c|}
\hline \multicolumn{7}{|c|}{ Dependent variable: thickness [mm] } \\
\hline \multicolumn{7}{|l|}{ Tukey HSD } \\
\hline \multirow[b]{2}{*}{ (I) assemblage } & \multirow[b]{2}{*}{$(\mathrm{J})$ assemblage } & \multirow{2}{*}{$\begin{array}{c}\text { Mean } \\
\text { difference } \\
(\mathrm{I}-\mathrm{J})\end{array}$} & \multirow[b]{2}{*}{ Std. error } & \multirow[b]{2}{*}{ Sig. } & \multicolumn{2}{|c|}{$95 \%$ Confidence interval } \\
\hline & & & & & $\begin{array}{l}\text { Lower } \\
\text { bound }\end{array}$ & $\begin{array}{l}\text { Upper } \\
\text { bound }\end{array}$ \\
\hline \multirow[t]{3}{*}{ Esztergom } & Arka & 1.09001 & 0.59963 & 0.268 & -0.4640 & 2.6441 \\
\hline & Bodrogkeresztúr & 1.62240 & 0.70458 & 0.101 & -0.2037 & 3.4485 \\
\hline & Nadap & 0.17412 & 0.60345 & 0.992 & -1.3898 & 1.7381 \\
\hline \multirow[t]{3}{*}{ Nadap } & Arka & 0.91589 & 0.40950 & 0.117 & -0.1454 & 1.9772 \\
\hline & Bodrogkeresztúr & 1.44828 & 0.55188 & 0.046 & 0.0180 & 2.8786 \\
\hline & Esztergom & -0.17412 & 0.60345 & 0.992 & -1.7381 & 1.3898 \\
\hline
\end{tabular}

Table 198. Regional blade thicknesses comparison between periods (complete specimens) with ANOVA and Tukey post hoc test

ANOVA

\begin{tabular}{|l|c|c|c|c|c|}
\hline Thickness [mm] & Sum of squares & df & Mean square & F & Sig. \\
\hline Between groups & 86.869 & 2 & 43.434 & 4.957 & 0.007 \\
\hline Within groups & 3749.986 & 428 & 8.762 & & \\
\hline Total & 3836.854 & 430 & & & \\
\hline
\end{tabular}

Multiple Comparisons

\begin{tabular}{|c|c|c|c|c|c|c|}
\hline \multirow{2}{*}{\multicolumn{7}{|c|}{\begin{tabular}{|l|} 
Dependent variable: thickness [mm] \\
Tukey HSD
\end{tabular}}} \\
\hline & & & & & & \\
\hline \multirow[b]{2}{*}{ (I) age } & \multirow[b]{2}{*}{ (J) age } & \multirow{2}{*}{$\begin{array}{c}\text { Mean } \\
\text { difference } \\
(\mathrm{I}-\mathrm{J})\end{array}$} & \multirow[b]{2}{*}{ Std. error } & \multirow[b]{2}{*}{ Sig. } & \multicolumn{2}{|c|}{$95 \%$ Confidence interval } \\
\hline & & & & & $\begin{array}{l}\text { Lower } \\
\text { bound }\end{array}$ & $\begin{array}{l}\text { Upper } \\
\text { bound }\end{array}$ \\
\hline \multirow[t]{2}{*}{ LG } & EEG & 0.85574 & 0.29629 & 0.011 & 0.1589 & 1.5526 \\
\hline & LEG & 1.11926 & 0.60301 & 0.153 & -0.2990 & 2.5375 \\
\hline \multirow[t]{2}{*}{ EEG } & LG & -0.85574 & 0.29629 & 0.011 & -1.5526 & -0.1589 \\
\hline & LEG & 0.26352 & 0.61089 & 0.903 & -1.1732 & 1.7003 \\
\hline \multirow[t]{2}{*}{ LEG } & LG & -1.11926 & 0.60301 & 0.153 & -2.5375 & 0.2990 \\
\hline & EEG & -0.26352 & 0.61089 & 0.903 & -1.7003 & 1.1732 \\
\hline
\end{tabular}


Table 199. Blade thicknesses comparison between periods (complete specimens) with ANOVA and Tukey post hoc test

ANOVA

\begin{tabular}{|l|c|c|c|c|c|}
\hline \multicolumn{7}{|l|}{ Thickness [mm] } & Sum of squares & df & Mean square & F & Sig. \\
\hline Between groups & 478.237 & 2 & 239.118 & 16.922 & 0.000 \\
\hline Within groups & 16095.026 & 1139 & 14.131 & & \\
\hline Total & 16573.263 & 1141 & & & \\
\hline
\end{tabular}

Multiple Comparisons

\begin{tabular}{|c|c|c|c|c|c|c|}
\hline \multirow{2}{*}{\multicolumn{7}{|c|}{$\begin{array}{l}\text { Dependent variable: thickness [mm] } \\
\text { Tukey HSD }\end{array}$}} \\
\hline \multirow[b]{2}{*}{ (I) age } & \multirow[b]{2}{*}{ (J) age } & & \multirow[b]{2}{*}{ Std. error } & \multirow[b]{2}{*}{ Sig. } & \multicolumn{2}{|c|}{ 95\% Confidence interval } \\
\hline & & $\begin{array}{c}\text { Mean } \\
\text { difference } \\
(\mathrm{I}-\mathrm{J})\end{array}$ & & & $\begin{array}{l}\text { Lower } \\
\text { bound }\end{array}$ & $\begin{array}{l}\text { Upper } \\
\text { bound }\end{array}$ \\
\hline \multirow[t]{2}{*}{ LG } & EEG & 1.39004 & 0.28954 & 0.000 & 0.7106 & 2.0695 \\
\hline & LEG & 1.47743 & 0.36643 & 0.000 & 0.6175 & 2.3374 \\
\hline \multirow[t]{2}{*}{ EEG } & $\mathrm{LG}$ & -1.39004 & 0.28954 & 0.000 & -2.0695 & -0.7106 \\
\hline & LEG & 0.08739 & 0.42793 & 0.977 & -0.9169 & 1.0916 \\
\hline \multirow[t]{2}{*}{ LEG } & LG & -1.47743 & 0.36643 & 0.000 & -2.3374 & -0.6175 \\
\hline & EEG & -0.08739 & 0.42793 & 0.977 & -1.0916 & 0.9169 \\
\hline
\end{tabular}

Table 200. Flake lengths comparison between assemblages (complete specimens) with ANOVA and Tukey post hoc test

ANOVA

\begin{tabular}{|l|c|c|c|c|c|}
\hline Length [mm] & Sum of squares & df & Mean square & F & Sig. \\
\hline Between groups & 205238.406 & 7 & 29319.772 & 117.111 & 0.000 \\
\hline Within groups & 593102.184 & 2369 & 250.360 & & \\
\hline Total & 798340.590 & 2376 & & & \\
\hline
\end{tabular}

Multiple Comparisons

\begin{tabular}{|c|c|c|c|c|c|c|}
\hline \multicolumn{7}{|c|}{ Dependent variable: length [mm] } \\
\hline \multirow[b]{2}{*}{ (I) assemblage } & \multirow[b]{2}{*}{ (J) assemblage } & \multirow{2}{*}{$\begin{array}{c}\text { Mean } \\
\text { difference } \\
(\mathrm{I}-\mathrm{J})\end{array}$} & \multirow[b]{2}{*}{ Std. error } & \multirow[b]{2}{*}{ Sig. } & \multicolumn{2}{|c|}{$95 \%$ Confidence interval } \\
\hline & & & & & $\begin{array}{l}\text { Lower } \\
\text { bound }\end{array}$ & $\begin{array}{l}\text { Upper } \\
\text { bound }\end{array}$ \\
\hline \multirow[t]{7}{*}{ Arka } & Bodrogkeresztúr & 10.21625 & 0.92347 & 0.000 & 7.4148 & 13.0177 \\
\hline & Corvin-tér & 21.17743 & 2.05990 & 0.000 & 14.9285 & 27.4263 \\
\hline & Esztergom & 15.15267 & 2.36559 & 0.000 & 7.9764 & 22.3289 \\
\hline & Hidasnémeti & 13.39903 & 1.45730 & 0.000 & 8.9782 & 17.8199 \\
\hline & Nadap & 22.64796 & 2.84481 & 0.000 & 14.0179 & 31.2780 \\
\hline & Ságvár & 21.61420 & 0.78699 & 0.000 & 19.2268 & 24.0016 \\
\hline & Sajószentpéter & 13.93871 & 3.14618 & 0.000 & 4.3945 & 23.4830 \\
\hline \multirow[t]{7}{*}{ Bodrogkeresztúr } & Arka & -10.21625 & 0.92347 & 0.000 & -13.0177 & -7.4148 \\
\hline & Corvin-tér & 10.96118 & 2.13484 & 0.000 & 4.4849 & 17.4374 \\
\hline & Esztergom & 4.93643 & 2.43113 & 0.461 & -2.4387 & 12.3115 \\
\hline & Hidasnémeti & 3.18278 & 1.56144 & 0.456 & -1.5540 & 7.9196 \\
\hline & Nadap & 12.43171 & 2.89954 & 0.000 & 3.6357 & 21.2278 \\
\hline & Ságvár & 11.39795 & 0.96630 & 0.000 & 8.4666 & 14.3293 \\
\hline & Sajószentpéter & 3.72246 & 3.19575 & 0.942 & -5.9722 & 13.4171 \\
\hline
\end{tabular}


Table 200. Continued

Multiple Comparisons

Dependent variable: length [mm]

Tukey HSD

\begin{tabular}{|c|c|c|c|c|c|c|}
\hline \multirow{2}{*}{ (I) assemblage } & \multirow[b]{2}{*}{ (J) assemblage } & \multirow{2}{*}{$\begin{array}{c}\text { Mean } \\
\text { difference } \\
(\mathrm{I}-\mathrm{J})\end{array}$} & \multirow[b]{2}{*}{ Std. error } & \multirow[b]{2}{*}{ Sig. } & \multicolumn{2}{|c|}{$95 \%$ Confidence interval } \\
\hline & & & & & $\begin{array}{l}\text { Lower } \\
\text { bound }\end{array}$ & $\begin{array}{l}\text { Upper } \\
\text { bound }\end{array}$ \\
\hline \multirow[t]{7}{*}{ Corvin-tér } & Arka & -21.17743 & 2.05990 & 0.000 & -27.4263 & -14.9285 \\
\hline & Bodrogkeresztúr & -10.96118 & 2.13484 & 0.000 & -17.4374 & -4.4849 \\
\hline & Esztergom & -6.02476 & 3.04972 & 0.499 & -15.2764 & 3.2269 \\
\hline & Hidasnémeti & -7.77840 & 2.41422 & 0.028 & -15.1022 & -0.4546 \\
\hline & Nadap & 1.47053 & 3.43478 & 1.000 & -8.9492 & 11.8903 \\
\hline & Ságvár & 0.43677 & 2.07945 & 1.000 & -5.8715 & 6.7450 \\
\hline & Sajószentpéter & -7.23872 & 3.68825 & 0.508 & -18.4274 & 3.9500 \\
\hline \multirow[t]{7}{*}{ Esztergom } & Arka & -15.15267 & 2.36559 & 0.000 & -22.3289 & -7.9764 \\
\hline & Bodrogkeresztúr & -4.93643 & 2.43113 & 0.461 & -12.3115 & 2.4387 \\
\hline & Corvin-tér & 6.02476 & 3.04972 & 0.499 & -3.2269 & 15.2764 \\
\hline & Hidasnémeti & -1.75365 & 2.67980 & 0.998 & -9.8831 & 6.3758 \\
\hline & Nadap & 7.49529 & 3.62637 & 0.437 & -3.5057 & 18.4962 \\
\hline & Ságvár & 6.46152 & 2.38264 & 0.119 & -0.7665 & 13.6895 \\
\hline & Sajószentpéter & -1.21397 & 3.86730 & 1.000 & -12.9458 & 10.5179 \\
\hline \multirow[t]{7}{*}{ Hidasnémeti } & Arka & -13.39903 & 1.45730 & 0.000 & -17.8199 & -8.9782 \\
\hline & Bodrogkeresztúr & -3.18278 & 1.56144 & 0.456 & -7.9196 & 1.5540 \\
\hline & Corvin-tér & 7.77840 & 2.41422 & 0.028 & 0.4546 & 15.1022 \\
\hline & Esztergom & 1.75365 & 2.67980 & 0.998 & -6.3758 & 9.8831 \\
\hline & Nadap & 9.24893 & 3.11099 & 0.060 & -0.1886 & 18.6864 \\
\hline & Ságvár & 8.21517 & 1.48481 & 0.000 & 3.7108 & 12.7195 \\
\hline & Sajószentpéter & 0.53968 & 3.38877 & 1.000 & -9.7405 & 10.8198 \\
\hline \multirow[t]{7}{*}{ Nadap } & Arka & -22.64796 & 2.84481 & 0.000 & -31.2780 & -14.0179 \\
\hline & Bodrogkeresztúr & -12.43171 & 2.89954 & 0.000 & -21.2278 & -3.6357 \\
\hline & Corvin-tér & -1.47053 & 3.43478 & 1.000 & -11.8903 & 8.9492 \\
\hline & Esztergom & -7.49529 & 3.62637 & 0.437 & -18.4962 & 3.5057 \\
\hline & Hidasnémeti & -9.24893 & 3.11099 & 0.060 & -18.6864 & 0.1886 \\
\hline & Ságvár & -1.03377 & 2.85900 & 1.000 & -9.7068 & 7.6393 \\
\hline & Sajószentpéter & -8.70925 & 4.17767 & 0.425 & -21.3827 & 3.9641 \\
\hline \multirow[t]{7}{*}{ Ságvár } & Arka & -21.61420 & 0.78699 & 0.000 & -24.0016 & -19.2268 \\
\hline & Bodrogkeresztúr & -11.39795 & 0.96630 & 0.000 & -14.3293 & -8.4666 \\
\hline & Corvin-tér & -0.43677 & 2.07945 & 1.000 & -6.7450 & 5.8715 \\
\hline & Esztergom & -6.46152 & 2.38264 & 0.119 & -13.6895 & 0.7665 \\
\hline & Hidasnémeti & -8.21517 & 1.48481 & 0.000 & -12.7195 & -3.7108 \\
\hline & Nadap & 1.03377 & 2.85900 & 1.000 & -7.6393 & 9.7068 \\
\hline & Sajószentpéter & -7.67549 & 3.15901 & 0.227 & -17.2587 & 1.9077 \\
\hline \multirow[t]{7}{*}{ Sajószentpéter } & Arka & -13.93871 & 3.14618 & 0.000 & -23.4830 & -4.3945 \\
\hline & Bodrogkeresztúr & -3.72246 & 3.19575 & 0.942 & -13.4171 & 5.9722 \\
\hline & Corvin-tér & 7.23872 & 3.68825 & 0.508 & -3.9500 & 18.4274 \\
\hline & Esztergom & 1.21397 & 3.86730 & 1.000 & -10.5179 & 12.9458 \\
\hline & Hidasnémeti & -0.53968 & 3.38877 & 1.000 & -10.8198 & 9.7405 \\
\hline & Nadap & 8.70925 & 4.17767 & 0.425 & -3.9641 & 21.3827 \\
\hline & Ságvár & 7.67549 & 3.15901 & 0.227 & -1.9077 & 17.2587 \\
\hline
\end{tabular}


Table 201. Flake lengths comparison between periods (complete specimens) with ANOVA and Tukey post hoc test

ANOVA

Length $[\mathrm{mm}]$

\begin{tabular}{|l|c|c|c|c|c|}
\hline & Sum of squares & df & Mean square & F & Sig. \\
\hline Between groups & 158354.008 & 2 & 79177.004 & 293.703 & 0.000 \\
\hline Within groups & 639986.582 & 2374 & 269.582 & & \\
\hline Total & 798340.590 & 2376 & & & \\
\hline
\end{tabular}

Multiple Comparisons

Dependent variable: length $[\mathrm{mm}]$

Tukey HSD

\begin{tabular}{|c|c|c|c|c|c|c|}
\hline \multirow[b]{2}{*}{ (I) age } & \multirow[b]{2}{*}{ (J) age } & \multirow{2}{*}{$\begin{array}{c}\text { Mean } \\
\text { difference } \\
(\mathrm{I}-\mathrm{J})\end{array}$} & \multirow[b]{2}{*}{ Std. error } & \multirow[b]{2}{*}{ Sig. } & \multicolumn{2}{|c|}{$95 \%$ Confidence interval } \\
\hline & & & & & $\begin{array}{l}\text { Lower } \\
\text { bound }\end{array}$ & $\begin{array}{l}\text { Upper } \\
\text { bound }\end{array}$ \\
\hline \multirow[t]{2}{*}{ LG } & EEG & 17.26696 & 0.72378 & 0.000 & 15.5696 & 18.9644 \\
\hline & LEG & 13.87687 & 1.89467 & 0.000 & 9.4335 & 18.3202 \\
\hline \multirow[t]{2}{*}{ EEG } & LG & -17.26696 & 0.72378 & 0.000 & -18.9644 & -15.5696 \\
\hline & LEG & -3.39009 & 1.93880 & 0.187 & -7.9369 & 1.1567 \\
\hline \multirow[t]{2}{*}{ LEG } & LG & -13.87687 & 1.89467 & 0.000 & -18.3202 & -9.4335 \\
\hline & EEG & 3.39009 & 1.93880 & 0.187 & -1.1567 & 7.9369 \\
\hline
\end{tabular}

Table 202. Regional flake lengths comparison within LG (complete specimens) with ANOVA and Tukey post hoc test

ANOVA

\begin{tabular}{|l|c|c|c|c|c|}
\hline Length [mm] & Sum of squares & df & Mean square & F & Sig. \\
\hline Between groups & 1122.153 & 3 & 374.051 & 2.360 & 0.072 \\
\hline Within groups & 46754.486 & 295 & 158.490 & & \\
\hline Total & 47876.639 & 298 & & & \\
\hline
\end{tabular}

Multiple Comparisons

Dependent variable: length [mm]

Tukey HSD

\begin{tabular}{|c|c|c|c|c|c|c|}
\hline \multirow[b]{2}{*}{ (I) assemblage } & \multirow[b]{2}{*}{ (J) assemblage } & \multirow{2}{*}{$\begin{array}{c}\text { Mean } \\
\text { difference } \\
(\mathrm{I}-\mathrm{J})\end{array}$} & \multirow[b]{2}{*}{ Std. error } & \multirow[b]{2}{*}{ Sig. } & \multicolumn{2}{|c|}{$95 \%$ Confidence interval } \\
\hline & & & & & $\begin{array}{l}\text { Lower } \\
\text { bound }\end{array}$ & $\begin{array}{l}\text { Upper } \\
\text { bound }\end{array}$ \\
\hline \multirow{3}{*}{ Arka } & Bodrogkeresztúr & 2.20595 & 2.28609 & 0.769 & -3.7007 & 8.1126 \\
\hline & Hidasnémeti & -2.97753 & 1.69652 & 0.297 & -7.3609 & 1.4058 \\
\hline & Sajószentpéter & -2.43785 & 2.79284 & 0.819 & -9.6538 & 4.7781 \\
\hline \multirow[t]{3}{*}{ Bodrogkeresztúr } & Arka & -2.20595 & 2.28609 & 0.769 & -8.1126 & 3.7007 \\
\hline & Hidasnémeti & -5.18348 & 2.16702 & 0.081 & -10.7825 & 0.4155 \\
\hline & Sajószentpéter & -4.64380 & 3.10125 & 0.440 & -12.6566 & 3.3690 \\
\hline \multirow[t]{3}{*}{ Hidasnémeti } & Arka & 2.97753 & 1.69652 & 0.297 & -1.4058 & 7.3609 \\
\hline & Bodrogkeresztúr & 5.18348 & 2.16702 & 0.081 & -0.4155 & 10.7825 \\
\hline & Sajószentpéter & 0.53968 & 2.69625 & 0.997 & -6.4267 & 7.5061 \\
\hline \multirow[t]{3}{*}{ Sajószentpéter } & Arka & 2.43785 & 2.79284 & 0.819 & -4.7781 & 9.6538 \\
\hline & Bodrogkeresztúr & 4.64380 & 3.10125 & 0.440 & -3.3690 & 12.6566 \\
\hline & Hidasnémeti & -0.53968 & 2.69625 & 0.997 & -7.5061 & 6.4267 \\
\hline
\end{tabular}


Table 203. Local flake lengths comparison between assemblages (complete specimens) with ANOVA and Tukey post hoc test

ANOVA

Length [mm]

\begin{tabular}{|l|c|c|c|c|c|}
\hline & Sum of squares & df & Mean square & F & Sig. \\
\hline Between groups & 28269.557 & 2 & 14134.779 & 41.684 & 0.000 \\
\hline Within groups & 391648.610 & 1155 & 339.090 & & \\
\hline Total & 419918.167 & 1157 & & & \\
\hline
\end{tabular}

Multiple Comparisons

Dependent variable: length [mm]

Tukey HSD

\begin{tabular}{|c|c|c|c|c|c|c|}
\hline \multirow[b]{2}{*}{ (I) assemblage } & \multirow[b]{2}{*}{ (J) assemblage } & \multirow{2}{*}{$\begin{array}{c}\text { Mean } \\
\text { difference } \\
(\mathrm{I}-\mathrm{J})\end{array}$} & \multirow[b]{2}{*}{ Std. error } & \multirow[b]{2}{*}{ Sig. } & \multicolumn{2}{|c|}{ 95\% Confidence interval } \\
\hline & & & & & $\begin{array}{l}\text { Lower } \\
\text { bound }\end{array}$ & $\begin{array}{l}\text { Upper } \\
\text { bound }\end{array}$ \\
\hline \multirow[t]{2}{*}{ Arka } & Bodrogkeresztúr & 10.67817 & 1.18054 & 0.000 & 7.9078 & 13.4486 \\
\hline & Corvin-tér & 9.44866 & 4.96471 & 0.138 & -2.2022 & 21.0995 \\
\hline \multirow[t]{2}{*}{ Bodrogkeresztúr } & Arka & -10.67817 & 1.18054 & 0.000 & -13.4486 & -7.9078 \\
\hline & Corvin-tér & -1.22951 & 5.01864 & 0.967 & -13.0069 & 10.5479 \\
\hline \multirow[t]{2}{*}{ Corvin-tér } & Arka & -9.44866 & 4.96471 & 0.138 & -21.0995 & 2.2022 \\
\hline & Bodrogkeresztúr & 1.22951 & 5.01864 & 0.967 & -10.5479 & 13.0069 \\
\hline
\end{tabular}

Table 204. Regional flake lengths comparison between assemblages (complete specimens) with ANOVA and Tukey post hoc test

ANOVA

\begin{tabular}{|l|c|r|c|c|c|}
\hline Length [mm] & Sum of squares & df & Mean square & F & Sig. \\
\hline Between groups & 20141.377 & 7 & 2877.340 & 26.528 & 0.000 \\
\hline Within groups & 94255.599 & 869 & 108.464 & & \\
\hline Total & 114396.976 & 876 & & & \\
\hline
\end{tabular}

Multiple Comparisons

Dependent variable: length [mm]

Tukey HSD

\begin{tabular}{|c|c|c|c|c|c|c|}
\hline \multirow[b]{2}{*}{ (I) assemblage } & \multirow[b]{2}{*}{$(\mathrm{J})$ assemblage } & \multirow{2}{*}{$\begin{array}{c}\text { Mean } \\
\text { difference } \\
(\mathrm{I}-\mathrm{J})\end{array}$} & \multirow[b]{2}{*}{ Std. error } & \multirow[b]{2}{*}{ Sig. } & \multicolumn{2}{|c|}{$95 \%$ Confidence interval } \\
\hline & & & & & $\begin{array}{l}\text { Lower } \\
\text { bound }\end{array}$ & $\begin{array}{l}\text { Upper } \\
\text { bound }\end{array}$ \\
\hline \multirow[t]{7}{*}{ Arka } & Bodrogkeresztúr & 2.20595 & 1.89119 & 0.941 & -3.5401 & 7.9520 \\
\hline & Corvin-tér & 8.85892 & 1.83844 & 0.000 & 3.2732 & 14.4447 \\
\hline & Esztergom & -10.05516 & 3.46594 & 0.074 & -20.5858 & 0.4754 \\
\hline & Hidasnémeti & -2.97753 & 1.40347 & 0.402 & -7.2417 & 1.2866 \\
\hline & Nadap & 5.70938 & 3.32065 & 0.675 & -4.3798 & 15.7985 \\
\hline & Ságvár & 7.98752 & 1.17465 & 0.000 & 4.4186 & 11.5565 \\
\hline & Sajószentpéter & -2.43785 & 2.31041 & 0.966 & -9.4576 & 4.5819 \\
\hline
\end{tabular}


Table 204. Continued

Multiple Comparisons

Dependent variable: length [mm]

Tukey HSD

\begin{tabular}{|c|c|c|c|c|c|c|}
\hline \multirow[b]{2}{*}{ (I) assemblage } & \multirow[b]{2}{*}{ (J) assemblage } & \multirow{2}{*}{$\begin{array}{c}\text { Mean } \\
\text { difference } \\
(\mathrm{I}-\mathrm{J})\end{array}$} & \multirow[b]{2}{*}{ Std. error } & \multirow[b]{2}{*}{ Sig. } & \multicolumn{2}{|c|}{$95 \%$ Confidence interval } \\
\hline & & & & & $\begin{array}{l}\text { Lower } \\
\text { bound }\end{array}$ & $\begin{array}{l}\text { Upper } \\
\text { bound }\end{array}$ \\
\hline \multirow[t]{7}{*}{ Bodrogkeresztúr } & Arka & -2.20595 & 1.89119 & 0.941 & -7.9520 & 3.5401 \\
\hline & Corvin-tér & 6.65297 & 2.15032 & 0.042 & 0.1196 & 13.1863 \\
\hline & Esztergom & -12.26111 & 3.64098 & 0.018 & -23.3236 & -1.1987 \\
\hline & Hidasnémeti & -5.18348 & 1.79270 & 0.076 & -10.6303 & 0.2633 \\
\hline & Nadap & 3.50343 & 3.50296 & 0.974 & -7.1396 & 14.1465 \\
\hline & Ságvár & 5.78157 & 1.61983 & 0.009 & 0.8600 & 10.7031 \\
\hline & Sajószentpéter & -4.64380 & 2.56555 & 0.613 & -12.4387 & 3.1511 \\
\hline \multirow[t]{7}{*}{ Corvin-tér } & Arka & -8.85892 & 1.83844 & 0.000 & -14.4447 & -3.2732 \\
\hline & Bodrogkeresztúr & -6.65297 & 2.15032 & 0.042 & -13.1863 & -0.1196 \\
\hline & Esztergom & -18.91408 & 3.61386 & 0.000 & -29.8941 & -7.9340 \\
\hline & Hidasnémeti & -11.83645 & 1.73695 & 0.000 & -17.1139 & -6.5591 \\
\hline & Nadap & -3.14954 & 3.47476 & 0.985 & -13.7069 & 7.4079 \\
\hline & Ságvár & -0.87140 & 1.55791 & 0.999 & -5.6048 & 3.8620 \\
\hline & Sajószentpéter & -11.29677 & 2.52691 & 0.000 & -18.9743 & -3.6192 \\
\hline \multirow[t]{7}{*}{ Esztergom } & Arka & 10.05516 & 3.46594 & 0.074 & -0.4754 & 20.5858 \\
\hline & Bodrogkeresztúr & 12.26111 & 3.64098 & 0.018 & 1.1987 & 23.3236 \\
\hline & Corvin-tér & 18.91408 & 3.61386 & 0.000 & 7.9340 & 29.8941 \\
\hline & Hidasnémeti & 7.07763 & 3.41319 & 0.433 & -3.2927 & 17.4480 \\
\hline & Nadap & 15.76455 & 4.55048 & 0.013 & 1.9388 & 29.5903 \\
\hline & Ságvár & 18.04268 & 3.32565 & 0.000 & 7.9383 & 28.1470 \\
\hline & Sajószentpéter & 7.61731 & 3.87533 & 0.506 & -4.1571 & 19.3918 \\
\hline \multirow{7}{*}{ Hidasnémeti } & Arka & 2.97753 & 1.40347 & 0.402 & -1.2866 & 7.2417 \\
\hline & Bodrogkeresztúr & 5.18348 & 1.79270 & 0.076 & -0.2633 & 10.6303 \\
\hline & Corvin-tér & 11.83645 & 1.73695 & 0.000 & 6.5591 & 17.1139 \\
\hline & Esztergom & -7.07763 & 3.41319 & 0.433 & -17.4480 & 3.2927 \\
\hline & Nadap & 8.68692 & 3.26555 & 0.136 & -1.2349 & 18.6087 \\
\hline & Ságvár & 10.96505 & 1.00844 & 0.000 & 7.9011 & 14.0290 \\
\hline & Sajószentpéter & 0.53968 & 2.23050 & 1.000 & -6.2373 & 7.3166 \\
\hline \multirow[t]{7}{*}{ Nadap } & Arka & -5.70938 & 3.32065 & 0.675 & -15.7985 & 4.3798 \\
\hline & Bodrogkeresztúr & -3.50343 & 3.50296 & 0.974 & -14.1465 & 7.1396 \\
\hline & Corvin-tér & 3.14954 & 3.47476 & 0.985 & -7.4079 & 13.7069 \\
\hline & Esztergom & -15.76455 & 4.55048 & 0.013 & -29.5903 & -1.9388 \\
\hline & Hidasnémeti & -8.68692 & 3.26555 & 0.136 & -18.6087 & 1.2349 \\
\hline & Ságvár & 2.27813 & 3.17394 & 0.996 & -7.3653 & 11.9216 \\
\hline & Sajószentpéter & -8.14724 & 3.74595 & 0.368 & -19.5286 & 3.2341 \\
\hline \multirow[t]{7}{*}{ Ságvár } & Arka & -7.98752 & 1.17465 & 0.000 & -11.5565 & -4.4186 \\
\hline & Bodrogkeresztúr & -5.78157 & 1.61983 & 0.009 & -10.7031 & -0.8600 \\
\hline & Corvin-tér & 0.87140 & 1.55791 & 0.999 & -3.8620 & 5.6048 \\
\hline & Esztergom & -18.04268 & 3.32565 & 0.000 & -28.1470 & -7.9383 \\
\hline & Hidasnémeti & -10.96505 & 1.00844 & 0.000 & -14.0290 & -7.9011 \\
\hline & Nadap & -2.27813 & 3.17394 & 0.996 & -11.9216 & 7.3653 \\
\hline & Sajószentpéter & -10.42537 & 2.09409 & 0.000 & -16.7879 & -4.0629 \\
\hline \multirow[t]{7}{*}{ Sajószentpéter } & Arka & 2.43785 & 2.31041 & 0.966 & -4.5819 & 9.4576 \\
\hline & Bodrogkeresztúr & 4.64380 & 2.56555 & 0.613 & -3.1511 & 12.4387 \\
\hline & Corvin-tér & 11.29677 & 2.52691 & 0.000 & 3.6192 & 18.9743 \\
\hline & Esztergom & -7.61731 & 3.87533 & 0.506 & -19.3918 & 4.1571 \\
\hline & Hidasnémeti & -0.53968 & 2.23050 & 1.000 & -7.3166 & 6.2373 \\
\hline & Nadap & 8.14724 & 3.74595 & 0.368 & -3.2341 & 19.5286 \\
\hline & Ságvár & 10.42537 & 2.09409 & 0.000 & 4.0629 & 16.7879 \\
\hline
\end{tabular}


Table 205. Transcarpathian flake lengths comparison between assemblages (complete specimens) with ANOVA and Tukey post hoc test

ANOVA

\begin{tabular}{|l|c|c|c|c|c|}
\hline Length [mm] & Sum of squares & df & Mean square & F & Sig. \\
\hline Between groups & 3454.263 & 4 & 863.566 & 6.296 & .000 \\
\hline Within groups & 18789.952 & 137 & 137.153 & & \\
\hline Total & 22244.215 & 141 & & & \\
\hline
\end{tabular}

Multiple Comparisons

\begin{tabular}{|c|c|c|c|c|c|c|}
\hline \multicolumn{7}{|c|}{ Dependent variable: length [mm] } \\
\hline \multicolumn{7}{|c|}{ Tukey HSD } \\
\hline \multirow[b]{2}{*}{ (I) assemblage } & \multirow[b]{2}{*}{$(\mathrm{J})$ assemblage } & \multirow{2}{*}{$\begin{array}{c}\text { Mean } \\
\text { difference } \\
(\mathrm{I}-\mathrm{J})\end{array}$} & \multirow[b]{2}{*}{ Std. error } & \multirow[b]{2}{*}{ Sig. } & \multicolumn{2}{|c|}{ 95\% Confidence interval } \\
\hline & & & & & $\begin{array}{l}\text { Lower } \\
\text { bound }\end{array}$ & $\begin{array}{l}\text { Upper } \\
\text { bound }\end{array}$ \\
\hline \multirow[t]{4}{*}{ Arka } & Bodrogkeresztúr & 10.23182 & 2.69690 & 0.002 & 2.7764 & 17.6872 \\
\hline & Esztergom & 7.55811 & 2.61227 & 0.035 & 0.3366 & 14.7796 \\
\hline & Nadap & 12.96095 & 3.10616 & 0.001 & 4.3742 & 21.5478 \\
\hline & Ságvár & 12.32143 & 4.76554 & 0.079 & -0.8527 & 25.4955 \\
\hline \multirow[t]{4}{*}{ Bodrogkeresztúr } & Arka & -10.23182 & 2.69690 & 0.002 & -17.6872 & -2.7764 \\
\hline & Esztergom & -2.67371 & 2.80410 & 0.875 & -10.4255 & 5.0781 \\
\hline & Nadap & 2.72913 & 3.26913 & 0.919 & -6.3082 & 11.7665 \\
\hline & Ságvár & 2.08961 & 4.87334 & 0.993 & -11.3825 & 15.5617 \\
\hline \multirow[t]{4}{*}{ Esztergom } & Arka & -7.55811 & 2.61227 & 0.035 & -14.7796 & -0.3366 \\
\hline & Bodrogkeresztúr & 2.67371 & 2.80410 & 0.875 & -5.0781 & 10.4255 \\
\hline & Nadap & 5.40284 & 3.19968 & 0.444 & -3.4425 & 14.2482 \\
\hline & Ságvár & 4.76332 & 4.82702 & 0.861 & -8.5807 & 18.1074 \\
\hline \multirow[t]{4}{*}{ Nadap } & Arka & -12.96095 & 3.10616 & 0.001 & -21.5478 & -4.3742 \\
\hline & Bodrogkeresztúr & -2.72913 & 3.26913 & 0.919 & -11.7665 & 6.3082 \\
\hline & Esztergom & -5.40284 & 3.19968 & 0.444 & -14.2482 & 3.4425 \\
\hline & Ságvár & -0.63952 & 5.11120 & 1.000 & -14.7692 & 13.4901 \\
\hline \multirow[t]{4}{*}{ Ságvár } & Arka & -12.32143 & 4.76554 & 0.079 & -25.4955 & 0.8527 \\
\hline & Bodrogkeresztúr & -2.08961 & 4.87334 & 0.993 & -15.5617 & 11.3825 \\
\hline & Esztergom & -4.76332 & 4.82702 & 0.861 & -18.1074 & 8.5807 \\
\hline & Nadap & 0.63952 & 5.11120 & 1.000 & -13.4901 & 14.7692 \\
\hline
\end{tabular}


Table 206. Flake domestic tool lengths comparison between assemblages (complete specimens) with ANOVA and Tukey post hoc test

ANOVA

\begin{tabular}{|l|c|c|c|c|c|}
\hline Length [mm] & Sum of squares & df & Mean square & F & Sig. \\
\hline Between groups & 17245.894 & 7 & 2463.699 & 10.502 & 0.000 \\
\hline Within groups & 105567.626 & 450 & 234.595 & & \\
\hline Total & 122813.520 & 457 & & & \\
\hline
\end{tabular}

Multiple Comparisons

\begin{tabular}{|c|c|c|c|c|c|c|}
\hline \multicolumn{7}{|c|}{ Dependent variable: length [mm] } \\
\hline \multicolumn{7}{|l|}{ Tukey HSD } \\
\hline \multirow[b]{2}{*}{ (I) assemblage } & \multirow[b]{2}{*}{ (J) assemblage } & \multirow{2}{*}{$\begin{array}{c}\text { Mean } \\
\text { difference } \\
(\mathrm{I}-\mathrm{J})\end{array}$} & \multirow[b]{2}{*}{ Std. error } & \multirow[b]{2}{*}{ Sig. } & \multicolumn{2}{|c|}{ 95\% Confidence interval } \\
\hline & & & & & $\begin{array}{l}\text { Lower } \\
\text { bound }\end{array}$ & $\begin{array}{l}\text { Upper } \\
\text { bound }\end{array}$ \\
\hline \multirow[t]{7}{*}{ Arka } & Bodrogkeresztúr & 0.46021 & 2.26550 & 1.000 & -6.4388 & 7.3592 \\
\hline & Corvin-tér & 13.19549 & 4.81235 & 0.113 & -1.4593 & 27.8503 \\
\hline & Esztergom & 0.74549 & 4.07948 & 1.000 & -11.6776 & 13.1685 \\
\hline & Hidasnémeti & -5.85466 & 3.77790 & 0.780 & -17.3593 & 5.6500 \\
\hline & Nadap & 16.25715 & 7.89036 & 0.442 & -7.7710 & 40.2853 \\
\hline & Ságvár & 11.60736 & 2.23918 & 0.000 & 4.7885 & 18.4262 \\
\hline & Sajószentpéter & -3.03222 & 4.27450 & 0.997 & -16.0491 & 9.9847 \\
\hline \multirow[t]{7}{*}{ Bodrogkeresztúr } & Arka & -0.46021 & 2.26550 & 1.000 & -7.3592 & 6.4388 \\
\hline & Corvin-tér & 12.73528 & 4.59052 & 0.104 & -1.2440 & 26.7146 \\
\hline & Esztergom & 0.28528 & 3.81528 & 1.000 & -11.3332 & 11.9038 \\
\hline & Hidasnémeti & -6.31487 & 3.49095 & 0.614 & -16.9457 & 4.3159 \\
\hline & Nadap & 15.79695 & 7.75706 & 0.458 & -7.8252 & 39.4191 \\
\hline & Ságvár & 11.14716 & 1.71117 & 0.000 & 5.9362 & 16.3581 \\
\hline & Sajószentpéter & -3.49243 & 4.02312 & 0.989 & -15.7438 & 8.7590 \\
\hline \multirow[t]{7}{*}{ Corvin-tér } & Arka & -13.19549 & 4.81235 & 0.113 & -27.8503 & 1.4593 \\
\hline & Bodrogkeresztúr & -12.73528 & 4.59052 & 0.104 & -26.7146 & 1.2440 \\
\hline & Esztergom & -12.45000 & 5.70812 & 0.365 & -29.8326 & 4.9326 \\
\hline & Hidasnémeti & -19.05015 & 5.49663 & 0.013 & -35.7888 & -2.3115 \\
\hline & Nadap & 3.06167 & 8.84298 & 1.000 & -23.8674 & 29.9907 \\
\hline & Ságvár & -1.58812 & 4.57759 & 1.000 & -15.5280 & 12.3518 \\
\hline & Sajószentpéter & -16.22771 & 5.84908 & 0.104 & -34.0396 & 1.5842 \\
\hline \multirow[t]{7}{*}{ Esztergom } & Arka & -0.74549 & 4.07948 & 1.000 & -13.1685 & 11.6776 \\
\hline & Bodrogkeresztúr & -0.28528 & 3.81528 & 1.000 & -11.9038 & 11.3332 \\
\hline & Corvin-tér & 12.45000 & 5.70812 & 0.365 & -4.9326 & 29.8326 \\
\hline & Hidasnémeti & -6.60015 & 4.86790 & 0.876 & -21.4241 & 8.2238 \\
\hline & Nadap & 15.51167 & 8.46651 & 0.598 & -10.2710 & 41.2943 \\
\hline & Ságvár & 10.86188 & 3.79971 & 0.084 & -0.7092 & 22.4329 \\
\hline & Sajószentpéter & -3.77771 & 5.26262 & 0.996 & -19.8037 & 12.2483 \\
\hline \multirow[t]{7}{*}{ Hidasnémeti } & Arka & 5.85466 & 3.77790 & 0.780 & -5.6500 & 17.3593 \\
\hline & Bodrogkeresztúr & 6.31487 & 3.49095 & 0.614 & -4.3159 & 16.9457 \\
\hline & Corvin-tér & 19.05015 & 5.49663 & 0.013 & 2.3115 & 35.7888 \\
\hline & Esztergom & 6.60015 & 4.86790 & 0.876 & -8.2238 & 21.4241 \\
\hline & Nadap & 22.11182 & 8.32539 & 0.139 & -3.2411 & 47.4647 \\
\hline & Ságvár & 17.46203 & 3.47393 & 0.000 & 6.8831 & 28.0410 \\
\hline & Sajószentpéter & 2.82244 & 5.03245 & 0.999 & -12.5026 & 18.1475 \\
\hline
\end{tabular}


Table 206. Continued

Multiple Comparisons

\begin{tabular}{|c|c|c|c|c|c|c|}
\hline \multicolumn{7}{|c|}{ Dependent variable: length [mm] } \\
\hline \multicolumn{7}{|c|}{ Tukey HSD } \\
\hline \multirow[b]{2}{*}{ (I) assemblage } & \multirow[b]{2}{*}{ (J) assemblage } & \multirow{2}{*}{$\begin{array}{c}\text { Mean } \\
\text { difference } \\
(\mathrm{I}-\mathrm{J})\end{array}$} & \multirow[b]{2}{*}{ Std. error } & \multirow[b]{2}{*}{ Sig. } & \multicolumn{2}{|c|}{ 95\% Confidence interval } \\
\hline & & & & & $\begin{array}{l}\text { Lower } \\
\text { bound }\end{array}$ & $\begin{array}{l}\text { Upper } \\
\text { bound }\end{array}$ \\
\hline \multirow[t]{7}{*}{ Nadap } & Arka & -16.25715 & 7.89036 & 0.442 & -40.2853 & 7.7710 \\
\hline & Bodrogkeresztúr & -15.79695 & 7.75706 & 0.458 & -39.4191 & 7.8252 \\
\hline & Corvin-tér & -3.06167 & 8.84298 & 1.000 & -29.9907 & 23.8674 \\
\hline & Esztergom & -15.51167 & 8.46651 & 0.598 & -41.2943 & 10.2710 \\
\hline & Hidasnémeti & -22.11182 & 8.32539 & 0.139 & -47.4647 & 3.2411 \\
\hline & Ságvár & -4.64979 & 7.74942 & 0.999 & -28.2487 & 18.9491 \\
\hline & Sajószentpéter & -19.28938 & 8.56218 & 0.322 & -45.3633 & 6.7846 \\
\hline \multirow[t]{7}{*}{ Ságvár } & Arka & -11.60736 & 2.23918 & 0.000 & -18.4262 & -4.7885 \\
\hline & Bodrogkeresztúr & -11.14716 & 1.71117 & 0.000 & -16.3581 & -5.9362 \\
\hline & Corvin-tér & 1.58812 & 4.57759 & 1.000 & -12.3518 & 15.5280 \\
\hline & Esztergom & -10.86188 & 3.79971 & 0.084 & -22.4329 & 0.7092 \\
\hline & Hidasnémeti & -17.46203 & 3.47393 & 0.000 & -28.0410 & -6.8831 \\
\hline & Nadap & 4.64979 & 7.74942 & 0.999 & -18.9491 & 28.2487 \\
\hline & Sajószentpéter & -14.63958 & 4.00836 & 0.007 & -26.8460 & -2.4331 \\
\hline \multirow[t]{7}{*}{ Sajószentpéter } & Arka & 3.03222 & 4.27450 & 0.997 & -9.9847 & 16.0491 \\
\hline & Bodrogkeresztúr & 3.49243 & 4.02312 & 0.989 & -8.7590 & 15.7438 \\
\hline & Corvin-tér & 16.22771 & 5.84908 & 0.104 & -1.5842 & 34.0396 \\
\hline & Esztergom & 3.77771 & 5.26262 & 0.996 & -12.2483 & 19.8037 \\
\hline & Hidasnémeti & -2.82244 & 5.03245 & 0.999 & -18.1475 & 12.5026 \\
\hline & Nadap & 19.28938 & 8.56218 & 0.322 & -6.7846 & 45.3633 \\
\hline & Ságvár & 14.63958 & 4.00836 & 0.007 & 2.4331 & 26.8460 \\
\hline
\end{tabular}

Table 207. Blade domestic tool lengths comparison between assemblages (complete specimens) with ANOVA and Tukey post hoc test

ANOVA

\begin{tabular}{|l|c|c|c|c|c|}
\hline Length [mm] & Sum of squares & df & Mean square & F & Sig. \\
\hline Between groups & 21350.389 & 7 & 3050.056 & 8.216 & 0.000 \\
\hline Within groups & 213828.464 & 576 & 371.230 & & \\
\hline Total & 235178.853 & 583 & & & \\
\hline
\end{tabular}

Multiple Comparisons

Dependent variable: length [mm]

Tukey HSD

\begin{tabular}{|c|c|c|c|c|c|c|}
\hline \multirow[b]{2}{*}{ (I) assemblage } & \multirow[b]{2}{*}{ (J) assemblage } & \multirow{2}{*}{$\begin{array}{c}\text { Mean } \\
\text { difference } \\
(\mathrm{I}-\mathrm{J})\end{array}$} & \multirow[b]{2}{*}{ Std. error } & \multirow[b]{2}{*}{ Sig. } & \multicolumn{2}{|c|}{$95 \%$ Confidence interval } \\
\hline & & & & & $\begin{array}{l}\text { Lower } \\
\text { bound }\end{array}$ & $\begin{array}{l}\text { Upper } \\
\text { bound }\end{array}$ \\
\hline \multirow{7}{*}{ Arka } & Bodrogkeresztúr & -5.06483 & 2.29891 & 0.351 & -12.0583 & 1.9287 \\
\hline & Corvin-tér & 14.70383 & 6.08314 & 0.235 & -3.8017 & 33.2093 \\
\hline & Esztergom & 1.41891 & 2.70848 & 1.000 & -6.8205 & 9.6584 \\
\hline & Hidasnémeti & -4.08833 & 2.86464 & 0.844 & -12.8028 & 4.6262 \\
\hline & Nadap & -8.77660 & 5.29198 & 0.714 & -24.8753 & 7.3221 \\
\hline & Ságvár & 11.28926 & 2.88821 & 0.003 & 2.5030 & 20.0755 \\
\hline & Sajószentpéter & -11.29708 & 4.48670 & 0.190 & -24.9461 & 2.3519 \\
\hline
\end{tabular}


Table 207. Continued

Multiple Comparisons

Dependent variable: length [mm]

Tukey HSD

\begin{tabular}{|c|c|c|c|c|c|c|}
\hline \multirow[b]{2}{*}{ (I) assemblage } & \multirow[b]{2}{*}{ (J) assemblage } & \multirow{2}{*}{$\begin{array}{c}\text { Mean } \\
\text { difference } \\
(\mathrm{I}-\mathrm{J})\end{array}$} & \multirow[b]{2}{*}{ Std. error } & \multirow[b]{2}{*}{ Sig. } & \multicolumn{2}{|c|}{ 95\% Confidence interval } \\
\hline & & & & & $\begin{array}{l}\text { Lower } \\
\text { bound }\end{array}$ & $\begin{array}{l}\text { Upper } \\
\text { bound }\end{array}$ \\
\hline \multirow[t]{7}{*}{ Bodrogkeresztúr } & Arka & 5.06483 & 2.29891 & 0.351 & -1.9287 & 12.0583 \\
\hline & Corvin-tér & 19.76865 & 5.98137 & 0.022 & 1.5727 & 37.9646 \\
\hline & Esztergom & 6.48374 & 2.47144 & 0.150 & -1.0346 & 14.0021 \\
\hline & Hidasnémeti & 0.97650 & 2.64165 & 1.000 & -7.0597 & 9.0127 \\
\hline & Nadap & -3.71177 & 5.17467 & 0.996 & -19.4536 & 12.0301 \\
\hline & Ságvár & 16.35408 & 2.66719 & 0.000 & 8.2402 & 24.4680 \\
\hline & Sajószentpéter & -6.23226 & 4.34772 & 0.841 & -19.4585 & 6.9939 \\
\hline \multirow[t]{7}{*}{ Corvin-tér } & Arka & -14.70383 & 6.08314 & 0.235 & -33.2093 & 3.8017 \\
\hline & Bodrogkeresztúr & -19.76865 & 5.98137 & 0.022 & -37.9646 & -1.5727 \\
\hline & Esztergom & -13.28492 & 6.15042 & 0.378 & -31.9951 & 5.4253 \\
\hline & Hidasnémeti & -18.79216 & 6.22076 & 0.053 & -37.7163 & 0.1320 \\
\hline & Nadap & -23.48042 & 7.64832 & 0.046 & -46.7474 & -0.2135 \\
\hline & Ságvár & -3.41457 & 6.23165 & 0.999 & -22.3719 & 15.5427 \\
\hline & Sajószentpéter & -26.00091 & 7.11493 & 0.007 & -47.6452 & -4.3566 \\
\hline \multirow[t]{7}{*}{ Esztergom } & Arka & -1.41891 & 2.70848 & 1.000 & -9.6584 & 6.8205 \\
\hline & Bodrogkeresztúr & -6.48374 & 2.47144 & 0.150 & -14.0021 & 1.0346 \\
\hline & Corvin-tér & 13.28492 & 6.15042 & 0.378 & -5.4253 & 31.9951 \\
\hline & Hidasnémeti & -5.50724 & 3.00486 & 0.598 & -14.6483 & 3.6338 \\
\hline & Nadap & -10.19551 & 5.36918 & 0.552 & -26.5291 & 6.1381 \\
\hline & Ságvár & 9.87034 & 3.02734 & 0.026 & 0.6609 & 19.0798 \\
\hline & Sajószentpéter & -12.71599 & 4.57750 & 0.103 & -26.6412 & 1.2092 \\
\hline \multirow[t]{7}{*}{ Hidasnémeti } & Arka & 4.08833 & 2.86464 & 0.844 & -4.6262 & 12.8028 \\
\hline & Bodrogkeresztúr & -0.97650 & 2.64165 & 1.000 & -9.0127 & 7.0597 \\
\hline & Corvin-tér & 18.79216 & 6.22076 & 0.053 & -0.1320 & 37.7163 \\
\hline & Esztergom & 5.50724 & 3.00486 & 0.598 & -3.6338 & 14.6483 \\
\hline & Nadap & -4.68827 & 5.44962 & 0.989 & -21.2666 & 11.8900 \\
\hline & Ságvár & 15.37759 & 3.16782 & 0.000 & 5.7408 & 25.0144 \\
\hline & Sajószentpéter & -7.20875 & 4.67160 & 0.784 & -21.4202 & 7.0027 \\
\hline \multirow[t]{7}{*}{ Nadap } & Arka & 8.77660 & 5.29198 & 0.714 & -7.3221 & 24.8753 \\
\hline & Bodrogkeresztúr & 3.71177 & 5.17467 & 0.996 & -12.0301 & 19.4536 \\
\hline & Corvin-tér & 23.48042 & 7.64832 & 0.046 & 0.2135 & 46.7474 \\
\hline & Esztergom & 10.19551 & 5.36918 & 0.552 & -6.1381 & 26.5291 \\
\hline & Hidasnémeti & 4.68827 & 5.44962 & 0.989 & -11.8900 & 21.2666 \\
\hline & Ságvár & 20.06585 & 5.46205 & 0.006 & 3.4497 & 36.6820 \\
\hline & Sajószentpéter & -2.52048 & 6.45157 & 1.000 & -22.1468 & 17.1058 \\
\hline \multirow[t]{7}{*}{ Ságvár } & Arka & -11.28926 & 2.88821 & 0.003 & -20.0755 & -2.5030 \\
\hline & Bodrogkeresztúr & -16.35408 & 2.66719 & 0.000 & -24.4680 & -8.2402 \\
\hline & Corvin-tér & 3.41457 & 6.23165 & 0.999 & -15.5427 & 22.3719 \\
\hline & Esztergom & -9.87034 & 3.02734 & 0.026 & -19.0798 & -0.6609 \\
\hline & Hidasnémeti & -15.37759 & 3.16782 & 0.000 & -25.0144 & -5.7408 \\
\hline & Nadap & -20.06585 & 5.46205 & 0.006 & -36.6820 & -3.4497 \\
\hline & Sajószentpéter & -22.58634 & 4.68609 & 0.000 & -36.8419 & -8.3308 \\
\hline \multirow[t]{7}{*}{ Sajószentpéter } & Arka & 11.29708 & 4.48670 & 0.190 & -2.3519 & 24.9461 \\
\hline & Bodrogkeresztúr & 6.23226 & 4.34772 & 0.841 & -6.9939 & 19.4585 \\
\hline & Corvin-tér & 26.00091 & 7.11493 & 0.007 & 4.3566 & 47.6452 \\
\hline & Esztergom & 12.71599 & 4.57750 & 0.103 & -1.2092 & 26.6412 \\
\hline & Hidasnémeti & 7.20875 & 4.67160 & 0.784 & -7.0027 & 21.4202 \\
\hline & Nadap & 2.52048 & 6.45157 & 1.000 & -17.1058 & 22.1468 \\
\hline & Ságvár & 22.58634 & 4.68609 & 0.000 & 8.3308 & 36.8419 \\
\hline
\end{tabular}


Table 208. Backed bladelet lengths comparison between assemblages (complete specimens) with ANOVA and Tukey post hoc test

ANOVA

\begin{tabular}{|l|c|c|c|c|c|}
\hline Length [mm] & Sum of squares & df & Mean square & F & Sig. \\
\hline Between groups & 2551.548 & 6 & 425.258 & 3.275 & 0.004 \\
\hline Within groups & 29991.577 & 231 & 129.834 & & \\
\hline Total & 32543.125 & 237 & & & \\
\hline
\end{tabular}

Multiple Comparisons

Dependent variable: length [mm]

Tukey HSD

\begin{tabular}{|c|c|c|c|c|c|c|}
\hline \multirow{2}{*}{ (I) assemblage } & \multirow[b]{2}{*}{ (J) assemblage } & \multirow{2}{*}{$\begin{array}{c}\text { Mean } \\
\text { difference } \\
(\mathrm{I}-\mathrm{J})\end{array}$} & \multirow[b]{2}{*}{ Std. error } & \multirow[b]{2}{*}{ Sig. } & \multicolumn{2}{|c|}{$95 \%$ Confidence interval } \\
\hline & & & & & $\begin{array}{l}\text { Lower } \\
\text { bound }\end{array}$ & $\begin{array}{l}\text { Upper } \\
\text { bound }\end{array}$ \\
\hline \multirow[t]{6}{*}{ Arka } & Bodrogkeresztúr & 5.03333 & 4.28872 & 0.903 & -7.7235 & 17.7902 \\
\hline & Corvin-tér & 0.28333 & 6.97765 & 1.000 & -20.4718 & 21.0385 \\
\hline & Esztergom & -5.11526 & 2.51477 & 0.396 & -12.5955 & 2.3650 \\
\hline & Hidasnémeti & -3.41667 & 3.67755 & 0.968 & -14.3556 & 7.5222 \\
\hline & Nadap & 0.33028 & 3.00270 & 1.000 & -8.6013 & 9.2619 \\
\hline & Ságvár & 6.46905 & 4.89463 & 0.841 & -8.0901 & 21.0282 \\
\hline \multirow[t]{6}{*}{ Bodrogkeresztúr } & Arka & -5.03333 & 4.28872 & 0.903 & -17.7902 & 7.7235 \\
\hline & Corvin-tér & -4.75000 & 7.50075 & 0.996 & -27.0611 & 17.5611 \\
\hline & Esztergom & -10.14859 & 3.72796 & 0.097 & -21.2375 & 0.9403 \\
\hline & Hidasnémeti & -8.45000 & 4.59325 & 0.523 & -22.1127 & 5.2127 \\
\hline & Nadap & -4.70306 & 4.07306 & 0.910 & -16.8184 & 7.4123 \\
\hline & Ságvár & 1.43571 & 5.61525 & 1.000 & -15.2669 & 18.1384 \\
\hline \multirow[t]{6}{*}{ Corvin-tér } & Arka & -0.28333 & 6.97765 & 1.000 & -21.0385 & 20.4718 \\
\hline & Bodrogkeresztúr & 4.75000 & 7.50075 & 0.996 & -17.5611 & 27.0611 \\
\hline & Esztergom & -5.39859 & 6.64772 & 0.984 & -25.1723 & 14.3752 \\
\hline & Hidasnémeti & -3.70000 & 7.16886 & 0.999 & -25.0239 & 17.6239 \\
\hline & Nadap & 0.04694 & 6.84722 & 1.000 & -20.3202 & 20.4141 \\
\hline & Ságvár & 6.18571 & 7.86292 & 0.986 & -17.2027 & 29.5741 \\
\hline \multirow[t]{6}{*}{ Esztergom } & Arka & 5.11526 & 2.51477 & 0.396 & -2.3650 & 12.5955 \\
\hline & Bodrogkeresztúr & 10.14859 & 3.72796 & 0.097 & -0.9403 & 21.2375 \\
\hline & Corvin-tér & 5.39859 & 6.64772 & 0.984 & -14.3752 & 25.1723 \\
\hline & Hidasnémeti & 1.69859 & 3.00482 & 0.998 & -7.2393 & 10.6365 \\
\hline & Nadap & 5.44554 & 2.12622 & 0.143 & -0.8789 & 11.7700 \\
\hline & Ságvár & 11.58431 & 4.41157 & 0.123 & -1.5380 & 24.7066 \\
\hline \multirow[t]{6}{*}{ Hidasnémeti } & Arka & 3.41667 & 3.67755 & 0.968 & -7.5222 & 14.3556 \\
\hline & Bodrogkeresztúr & 8.45000 & 4.59325 & 0.523 & -5.2127 & 22.1127 \\
\hline & Corvin-tér & 3.70000 & 7.16886 & 0.999 & -17.6239 & 25.0239 \\
\hline & Esztergom & -1.69859 & 3.00482 & 0.998 & -10.6365 & 7.2393 \\
\hline & Nadap & 3.74694 & 3.42361 & 0.929 & -6.4366 & 13.9305 \\
\hline & Ságvár & 9.88571 & 5.16355 & 0.473 & -5.4733 & 25.2448 \\
\hline \multirow[t]{6}{*}{ Nadap } & Arka & -0.33028 & 3.00270 & 1.000 & -9.2619 & 8.6013 \\
\hline & Bodrogkeresztúr & 4.70306 & 4.07306 & 0.910 & -7.4123 & 16.8184 \\
\hline & Corvin-tér & -0.04694 & 6.84722 & 1.000 & -20.4141 & 20.3202 \\
\hline & Esztergom & -5.44554 & 2.12622 & 0.143 & -11.7700 & 0.8789 \\
\hline & Hidasnémeti & -3.74694 & 3.42361 & 0.929 & -13.9305 & 6.4366 \\
\hline & Ságvár & 6.13877 & 4.70682 & 0.849 & -7.8617 & 20.1393 \\
\hline
\end{tabular}


Table 208. Continued

Multiple Comparisons

Dependent variable: length [mm]

Tukey HSD

\begin{tabular}{|c|c|c|c|c|c|c|}
\hline \multirow[b]{2}{*}{ (I) assemblage } & \multirow[b]{2}{*}{$(\mathrm{J})$ assemblage } & \multirow{2}{*}{$\begin{array}{c}\text { Mean } \\
\text { difference } \\
(\mathrm{I}-\mathrm{J})\end{array}$} & \multirow[b]{2}{*}{ Std. error } & \multirow[b]{2}{*}{ Sig. } & \multicolumn{2}{|c|}{$95 \%$ Confidence interval } \\
\hline & & & & & $\begin{array}{l}\text { Lower } \\
\text { bound }\end{array}$ & $\begin{array}{l}\text { Upper } \\
\text { bound }\end{array}$ \\
\hline \multirow[t]{6}{*}{ Ságvár } & Arka & -6.46905 & 4.89463 & 0.841 & -21.0282 & 8.0901 \\
\hline & Bodrogkeresztúr & -1.43571 & 5.61525 & 1.000 & -18.1384 & 15.2669 \\
\hline & Corvin-tér & -6.18571 & 7.86292 & 0.986 & -29.5741 & 17.2027 \\
\hline & Esztergom & -11.58431 & 4.41157 & 0.123 & -24.7066 & 1.5380 \\
\hline & Hidasnémeti & -9.88571 & 5.16355 & 0.473 & -25.2448 & 5.4733 \\
\hline & Nadap & -6.13877 & 4.70682 & 0.849 & -20.1393 & 7.8617 \\
\hline
\end{tabular}

Table 209. Backed bladelet widths comparison between assemblages (complete specimens) with ANOVA and Tukey post hoc test

ANOVA

Width [mm]

\begin{tabular}{|l|c|c|c|c|c|}
\hline & Sum of squares & df & Mean square & F & Sig. \\
\hline Between groups & 1576.446 & 6 & 262.741 & 31.299 & 0.000 \\
\hline Within groups & 1939.135 & 231 & 8.395 & & \\
\hline Total & 3515.581 & 237 & & & \\
\hline
\end{tabular}

Multiple Comparisons

Dependent variable: width [mm]

Tukey HSD

\begin{tabular}{|c|c|c|c|c|c|c|}
\hline \multirow[b]{2}{*}{ (I) assemblage } & \multirow[b]{2}{*}{ (J) assemblage } & \multirow{2}{*}{$\begin{array}{c}\text { Mean } \\
\text { difference } \\
(\mathrm{I}-\mathrm{J})\end{array}$} & \multirow[b]{2}{*}{ Std. error } & \multirow[b]{2}{*}{ Sig. } & \multicolumn{2}{|c|}{$95 \%$ Confidence interval } \\
\hline & & & & & $\begin{array}{l}\text { Lower } \\
\text { bound }\end{array}$ & $\begin{array}{l}\text { Upper } \\
\text { bound }\end{array}$ \\
\hline \multirow[t]{6}{*}{ Arka } & Bodrogkeresztúr & 1.25125 & 1.09052 & 0.913 & -1.9925 & 4.4950 \\
\hline & Corvin-tér & 1.29792 & 1.77425 & 0.991 & -3.9796 & 6.5754 \\
\hline & Esztergom & -5.53988 & 0.63944 & 0.000 & -7.4419 & -3.6378 \\
\hline & Hidasnémeti & -3.86875 & 0.93511 & 0.001 & -6.6502 & -1.0873 \\
\hline & Nadap & -0.92181 & 0.76351 & 0.891 & -3.1929 & 1.3493 \\
\hline & Ságvár & 1.24554 & 1.24458 & 0.953 & -2.4565 & 4.9476 \\
\hline \multirow[t]{6}{*}{ Bodrogkeresztúr } & Arka & -1.25125 & 1.09052 & 0.913 & -4.4950 & 1.9925 \\
\hline & Corvin-tér & 0.04667 & 1.90726 & 1.000 & -5.6265 & 5.7198 \\
\hline & Esztergom & -6.79113 & 0.94793 & 0.000 & -9.6108 & -3.9715 \\
\hline & Hidasnémeti & -5.12000 & 1.16795 & 0.000 & -8.5941 & -1.6459 \\
\hline & Nadap & -2.17306 & 1.03568 & 0.357 & -5.2537 & 0.9076 \\
\hline & Ságvár & -0.00571 & 1.42782 & 1.000 & -4.2528 & 4.2414 \\
\hline \multirow[t]{6}{*}{ Corvin-tér } & Arka & -1.29792 & 1.77425 & 0.991 & -6.5754 & 3.9796 \\
\hline & Bodrogkeresztúr & -0.04667 & 1.90726 & 1.000 & -5.7198 & 5.6265 \\
\hline & Esztergom & -6.83779 & 1.69035 & 0.001 & -11.8658 & -1.8098 \\
\hline & Hidasnémeti & -5.16667 & 1.82286 & 0.073 & -10.5888 & 0.2555 \\
\hline & Nadap & -2.21972 & 1.74108 & 0.863 & -7.3986 & 2.9591 \\
\hline & Ságvár & -0.05238 & 1.99935 & 1.000 & -5.9995 & 5.8947 \\
\hline
\end{tabular}


Table 209. Continued

Multiple Comparisons

\begin{tabular}{|c|c|c|c|c|c|c|}
\hline \multicolumn{7}{|c|}{ Dependent variable: width [mm] } \\
\hline \multicolumn{7}{|l|}{ Tukey HSD } \\
\hline \multirow[b]{2}{*}{ (I) assemblage } & \multirow[b]{2}{*}{$(\mathrm{J})$ assemblage } & \multirow{2}{*}{$\begin{array}{c}\text { Mean } \\
\text { difference } \\
(\mathrm{I}-\mathrm{J})\end{array}$} & \multirow[b]{2}{*}{ Std. error } & \multirow[b]{2}{*}{ Sig. } & \multicolumn{2}{|c|}{ 95\% Confidence interval } \\
\hline & & & & & $\begin{array}{l}\text { Lower } \\
\text { bound }\end{array}$ & $\begin{array}{l}\text { Upper } \\
\text { bound }\end{array}$ \\
\hline \multirow[t]{6}{*}{ Esztergom } & Arka & 5.53988 & 0.63944 & 0.000 & 3.6378 & 7.4419 \\
\hline & Bodrogkeresztúr & 6.79113 & 0.94793 & 0.000 & 3.9715 & 9.6108 \\
\hline & Corvin-tér & 6.83779 & 1.69035 & 0.001 & 1.8098 & 11.8658 \\
\hline & Hidasnémeti & 1.67113 & 0.76405 & 0.307 & -0.6016 & 3.9438 \\
\hline & Nadap & 4.61807 & 0.54065 & 0.000 & 3.0099 & 6.2262 \\
\hline & Ságvár & 6.78541 & 1.12175 & 0.000 & 3.4487 & 10.1221 \\
\hline \multirow[t]{6}{*}{ Hidasnémeti } & Arka & 3.86875 & 0.93511 & 0.001 & 1.0873 & 6.6502 \\
\hline & Bodrogkeresztúr & 5.12000 & 1.16795 & 0.000 & 1.6459 & 8.5941 \\
\hline & Corvin-tér & 5.16667 & 1.82286 & 0.073 & -0.2555 & 10.5888 \\
\hline & Esztergom & -1.67113 & 0.76405 & 0.307 & -3.9438 & 0.6016 \\
\hline & Nadap & 2.94694 & 0.87054 & 0.014 & 0.3575 & 5.5364 \\
\hline & Ságvár & 5.11429 & 1.31296 & 0.002 & 1.2089 & 9.0197 \\
\hline \multirow[t]{6}{*}{ Nadap } & Arka & 0.92181 & 0.76351 & 0.891 & -1.3493 & 3.1929 \\
\hline & Bodrogkeresztúr & 2.17306 & 1.03568 & 0.357 & -0.9076 & 5.2537 \\
\hline & Corvin-tér & 2.21972 & 1.74108 & 0.863 & -2.9591 & 7.3986 \\
\hline & Esztergom & -4.61807 & 0.54065 & 0.000 & -6.2262 & -3.0099 \\
\hline & Hidasnémeti & -2.94694 & 0.87054 & 0.014 & -5.5364 & -0.3575 \\
\hline & Ságvár & 2.16734 & 1.19683 & 0.542 & -1.3926 & 5.7273 \\
\hline \multirow[t]{6}{*}{ Ságvár } & Arka & -1.24554 & 1.24458 & 0.953 & -4.9476 & 2.4565 \\
\hline & Bodrogkeresztúr & 0.00571 & 1.42782 & 1.000 & -4.2414 & 4.2528 \\
\hline & Corvin-tér & 0.05238 & 1.99935 & 1.000 & -5.8947 & 5.9995 \\
\hline & Esztergom & -6.78541 & 1.12175 & 0.000 & -10.1221 & -3.4487 \\
\hline & Hidasnémeti & -5.11429 & 1.31296 & 0.002 & -9.0197 & -1.2089 \\
\hline & Nadap & -2.16734 & 1.19683 & 0.542 & -5.7273 & 1.3926 \\
\hline
\end{tabular}

Table 210. Backed bladelet thicknesses comparison between assemblages (complete specimens) with ANOVA and Tukey post hoc test

ANOVA

\begin{tabular}{|l|c|c|c|c|c|}
\hline \multicolumn{7}{|l|}{ Thickness [mm] } & Sum of squares & df & Mean square & F & Sig. \\
\hline Between groups & 105.832 & 6 & 17.639 & 10.740 & 0.000 \\
\hline Within groups & 379.381 & 231 & 1.642 & & \\
\hline Total & 485.214 & 237 & & & \\
\hline
\end{tabular}


Table 210. Continued

Multiple Comparisons

Dependent variable: thickness [mm]

Tukey HSD

\begin{tabular}{|c|c|c|c|c|c|c|}
\hline \multirow[b]{2}{*}{ (I) assemblage } & \multirow[b]{2}{*}{ (J) assemblage } & \multirow{2}{*}{$\begin{array}{c}\text { Mean } \\
\text { difference } \\
(\mathrm{I}-\mathrm{J})\end{array}$} & \multirow[b]{2}{*}{ Std. error } & \multirow[b]{2}{*}{ Sig. } & \multicolumn{2}{|c|}{$95 \%$ Confidence interval } \\
\hline & & & & & $\begin{array}{l}\text { Lower } \\
\text { bound }\end{array}$ & $\begin{array}{l}\text { Upper } \\
\text { bound }\end{array}$ \\
\hline \multirow[t]{6}{*}{ Arka } & Bodrogkeresztúr & 0.17250 & 0.48235 & 1.000 & -1.2623 & 1.6073 \\
\hline & Corvin-tér & 1.07917 & 0.78478 & 0.815 & -1.2552 & 3.4135 \\
\hline & Esztergom & -1.34947 & 0.28284 & 0.000 & -2.1908 & -0.5082 \\
\hline & Hidasnémeti & -1.65625 & 0.41362 & 0.002 & -2.8866 & -0.4259 \\
\hline & Nadap & -0.21778 & 0.33772 & 0.995 & -1.2223 & 0.7868 \\
\hline & Ságvár & 0.21250 & 0.55050 & 1.000 & -1.4250 & 1.8500 \\
\hline \multirow[t]{6}{*}{ Bodrogkeresztúr } & Arka & -0.17250 & 0.48235 & 1.000 & -1.6073 & 1.2623 \\
\hline & Corvin-tér & 0.90667 & 0.84361 & 0.935 & -1.6027 & 3.4160 \\
\hline & Esztergom & -1.52197 & 0.41929 & 0.006 & -2.7691 & -0.2748 \\
\hline & Hidasnémeti & -1.82875 & 0.51661 & 0.009 & -3.3654 & -0.2921 \\
\hline & Nadap & -0.39028 & 0.45810 & 0.979 & -1.7529 & 0.9723 \\
\hline & Ságvár & 0.04000 & 0.63155 & 1.000 & -1.8386 & 1.9186 \\
\hline \multirow[t]{6}{*}{ Corvin-tér } & Arka & -1.07917 & 0.78478 & 0.815 & -3.4135 & 1.2552 \\
\hline & Bodrogkeresztúr & -0.90667 & 0.84361 & 0.935 & -3.4160 & 1.6027 \\
\hline & Esztergom & -2.42864 & 0.74767 & 0.022 & -4.6526 & -0.2047 \\
\hline & Hidasnémeti & -2.73542 & 0.80628 & 0.014 & -5.1337 & -0.3371 \\
\hline & Nadap & -1.29694 & 0.77011 & 0.627 & -3.5876 & 0.9938 \\
\hline & Ságvár & -0.86667 & 0.88435 & 0.958 & -3.4972 & 1.7638 \\
\hline \multirow[t]{6}{*}{ Esztergom } & Arka & 1.34947 & 0.28284 & 0.000 & 0.5082 & 2.1908 \\
\hline & Bodrogkeresztúr & 1.52197 & 0.41929 & 0.006 & 0.2748 & 2.7691 \\
\hline & Corvin-tér & 2.42864 & 0.74767 & 0.022 & 0.2047 & 4.6526 \\
\hline & Hidasnémeti & -0.30678 & 0.33795 & 0.971 & -1.3120 & 0.6985 \\
\hline & Nadap & 1.13169 & 0.23914 & 0.000 & 0.4204 & 1.8430 \\
\hline & Ságvár & 1.56197 & 0.49617 & 0.030 & 0.0861 & 3.0378 \\
\hline \multirow[t]{6}{*}{ Hidasnémeti } & Arka & 1.65625 & 0.41362 & 0.002 & 0.4259 & 2.8866 \\
\hline & Bodrogkeresztúr & 1.82875 & 0.51661 & 0.009 & 0.2921 & 3.3654 \\
\hline & Corvin-tér & 2.73542 & 0.80628 & 0.014 & 0.3371 & 5.1337 \\
\hline & Esztergom & 0.30678 & 0.33795 & 0.971 & -0.6985 & 1.3120 \\
\hline & Nadap & 1.43847 & 0.38505 & 0.004 & 0.2931 & 2.5838 \\
\hline & Ságvár & 1.86875 & 0.58075 & 0.025 & 0.1413 & 3.5962 \\
\hline \multirow[t]{6}{*}{ Nadap } & Arka & 0.21778 & 0.33772 & 0.995 & -0.7868 & 1.2223 \\
\hline & Bodrogkeresztúr & 0.39028 & 0.45810 & 0.979 & -0.9723 & 1.7529 \\
\hline & Corvin-tér & 1.29694 & 0.77011 & 0.627 & -0.9938 & 3.5876 \\
\hline & Esztergom & -1.13169 & 0.23914 & 0.000 & -1.8430 & -0.4204 \\
\hline & Hidasnémeti & -1.43847 & 0.38505 & 0.004 & -2.5838 & -0.2931 \\
\hline & Ságvár & 0.43028 & 0.52938 & 0.983 & -1.1444 & 2.0049 \\
\hline \multirow[t]{6}{*}{ Ságvár } & Arka & -0.21250 & 0.55050 & 1.000 & -1.8500 & 1.4250 \\
\hline & Bodrogkeresztúr & -0.04000 & 0.63155 & 1.000 & -1.9186 & 1.8386 \\
\hline & Corvin-tér & 0.86667 & 0.88435 & 0.958 & -1.7638 & 3.4972 \\
\hline & Esztergom & -1.56197 & 0.49617 & 0.030 & -3.0378 & -0.0861 \\
\hline & Hidasnémeti & -1.86875 & 0.58075 & 0.025 & -3.5962 & -0.1413 \\
\hline & Nadap & -0.43028 & 0.52938 & 0.983 & -2.0049 & 1.1444 \\
\hline
\end{tabular}




\section{THE TECHNOLOGICAL DATA IN THE MUP AND LUP OF EASTERN CENTRAL EUROPE}

\section{The Late Gravettian period}

In Hungary, apart from what has been presented here, no complex lithic technology study was performed on the assemblages of the "Gravettian Entity". The often presented technological feature is the lithic raw material provenience (DoBOsI 2009c).

Among Late Gravettian sites not studied here, Hont-Parassa III (DoBosi, Simán 2003) yielded mostly regional materials and the assemblage contains several obsidian artifacts which show connection towards the Tokaj Mountains. The transcarpathian (TC) material group consisting of seven items makes up $0.5 \%$ of the assemblage. Pilisszántó I rockshelter (DовоSI, VöRös 1987) lower layer yielded 33\% of regional material and $22 \%$ of TC origin. There is a piece of obsidian in this layer that is of distant origin. The upper layer yielded only $14 \% \mathrm{TC}$ artifact and the rest was made of regional material. The material which cannot be sorted into any of the layers of Pilisszántó I rockshelter also is dominated by regional materials (77\%).

Outside the territory of Hungary, in the northern Carpathian basin, local and regional raw material use dominate the Late Gravettian assemblages, but TC materials also are present. Nitra-I Cěrmáň in Western Slovakia contained 5\% TC material and so did Cejkov I 6\% in Eastern Slovakia (KAMINSKÁ 2016). Nitra-I Cěrmáň also contained a piece of obsidian as an example for distant material (KAMINSKÁ, KozŁowsKi 2011). The highest TC material use (87.8\%) was recorded from the Moravany-Banka Horné fraské role shouldered point assemblage (KozıowsKi 2000). Trenčianske Bohuslavice assemblage contains $42.2 \%$ TC flints, and the rest of the material was made from the locally available radiolarite, and 56 items are of obsidian (ŽAÁr 2007). In eastern Slovakia, Kašov I lower layer yielded $49.3 \%$ of TC materials (Novík 2004) and the rest consists of mostly local, and in smaller percent regional lithic raw materials. This list showed that the TC use is more often greater at Slovakian than Hungarian sites, especially near the western periphery of the Carpathian basin.

In Lower Austria, Willendorf II layer 9 assemblage contains 55.2\% of TC materials and the rest was made of locally and regionally available radiolarite and different silicites (Oтте 1981). This pattern of raw material use is similar to what was found at the Váh valley sites. Grub-Kranawetberg main layer yielded mostly white patinated flint artifacts, part of which could have been derived from southern Moravian sources and from the erratic outcrops of Odera basin (ANTL-WEISER et al. 2010). The upper layer yielded mostly radiolarite artifacts.

In Moravia, Petřkovice I used chiefly local material (Novák 2008), which is Cretaceous flint at the Moravian Gate (PřICHYSTAL 2008). There are 17 items of radiolarite which seem to have been derived from the White Carpathians. It is of interest that the most complete shouldered point found in the 1953 excavation was made of radiolarite. The $55 \%$ of Milovice I lithic industry was made of regionally available radiolarite, $20 \%$ of the lithics are flints from the Moravian gate and the Kraków-Częstochowa 
Upland (Oliva 2009). Among the radiolarite pieces a few are of the Transdanubian Bakony type. Moreover, there are seven items of limnic silicites which might have derived from Central Slovakia or the Tokaj Mountains. Tokaj mountains connection was shown by the single piece of obsidian, as well.

In Poland, Jaksice II lithic tools were made of regional materials such as the Jurassic flint $38.9 \%$ and $51.6 \%$ of erratic cretaceous flint that might have been originated from Silesia (WILCZYŃSKi 2015, 2016). Also, the 4.4\% radiolarite material could have been collected from the Pieniny Klippen Belt outcrops in the Polish Carpathians. Kraków-Spadzista Late Gravettian hunters highly were local raw material users. Obsidian and radiolarite appear sporadically in at Spadzista (KozŁowski, SobCZYK 1987; WILCZYŃSKI 2015).

Going deeper into the lithic technology of the Late Gravettian, lesser data can be compared with the Hungarian sites. Universal feature of the lithic technology is the blade debitage, which is similar to what has been revealed from Hungary. Late Gravettian sites in Slovakia are dominated by unidirectional debitage (KozŁOWSKI 1998) and Trenčianske Bohuslavice is the only with more frequent use of opposite platform cores (ŽAÁR 2007). Plain platforms dominate the blades, and again Trenčianske Bohuslavice is the only with more prepared/faceted platform.

In Poland, Kraków-Spadzista layer 6 from areas B+B1 and C2 involved both the uni- and the bidirectional core reduction in the blade technology, and their proportions change from one excavated area to the other (WILCZYŃSKI 2016; WILCZYŃSKI et al. 2015). But, the blade platforms are also plain. Jaksice II chiefly performed unidirectional debitage and also plain platform preparation (WILCZYŃSKI 2015).

Milovice I technology in southern Moravia included mostly single platform cores but about $40 \%$ of all cores have two striking platforms (Oliva 2009). The blades of Milovice I have chiefly punctiform platforms. Petřkovice I (Novák 2008) showed the same features, but this industry contains the greatest amount of flake cores (11.6\%) among the Late Gravettian assemblages surveyed here, which is similar to Trenčianske Bohuslavice (10.7\%) (ŽAÁr 2007).

\section{The Early Epigravettian (Last Glacial Maximum)}

EE assemblages of Hungary are dominated by local or regional and distant raw materials. The proportion of TC material is 6\% at Mogyorósbánya, 3.5\% at Madaras, and none was found at Szob (Dobosi et al. 1989; Markó 2007; Dobosi 2016). The Mogyorósbánya TC material percent is higher than what was found at most LG sites except Bodrogkeresztúr, and the two EE sites, which was not predicted by present study based on the known raw material circulation in the Carpathian basin (LENGYEL 2014a). Szob and Mogyorósbánya also yielded a set of obsidian artifacts showing relations towards the Tokaj Mountains area (MARKó 2007, 2017). The Gravettian Entity Model describes Ságvár and the Ságvárian culture as Pebble Gravettian (DoBOsi 2016). At Ságvár, although pebble raw material is abundant, it far does not dominate the whole assemblage (LENGYEL 2011). The pebbles are chiefly of radiolarite, mostly Bakony types, 
which seem to be the closest raw material source to Ságvár. Among Ságvárian sites not analysed here, Mogyorósbánya and Szob were presented as pebble consumers (MARKó 2007, 2011; Doвosi 2016). The exploitation of pebbles at these sites seems to be related with the fact that this raw material was the closest available to the knappers. Also, the observations of Doвosi (2016) on the low blade frequency and short size of the blades suits the technological features of EE assemblages studied here. Indeed, Ságvár huntergatherers never were able to produce most of their blades long from the small sized pebble materials, but from the distant raw materials they made larger blades. The pebble use in the Ságvárian seems rather a Transdanubian lithic resource constrain observed also from the Lower Palaeolithic (Dовоsi 2016). Among the sites which have been reclassified as Early Epigravettian on the basis of their typological features (LENGYEL 2016), at Jászfelsőszentgyörgy the lithic raw material usage is similar to Ságvár with regional and distant materials (PRISKIN 2011). The proportions of the Bükk and Tokaj Mountains raw materials are greater in this assemblage due to the presence meta-rhyolite, obsidian and limnic silicite, all together $27.4 \%$. The ratio of TC materials is $1.66 \%$ that is similarly low in EE assemblages. In the Pilismarót site cluster the lithic raw material composition in details was not published (DoBosi 2006, 2014), but it is known that there are TC materials in these assemblages.

Early Epigravettian lithic technology data, compared to the Late Gravettian, is less abundant due to that most of the sites are found in Hungary and their technological studies have not yet been performed. As far as the raw material concerned, Kašov I upper layer in eastern Slovakia used mainly the local obsidian sources (BÁNESZ et al. 1992). Grubgraben, Lower Austria, in layers 1 and 2 regional material dominate and TC materials make up less than 5\%. In layers 3 and 4, however, the TC material proportion grows up to $23-24 \%$ on the expense of the regional materials (MONTET-WHITE 1990). This elevated frequency of TC material is the highest among all Last Glacial Maximum (LGM) EE sites. Stránská skála IV processed regional and local materials the most, and TC material makes up $2.1 \%$ of the lithic assemblage. A single piece of obsidian in this industry shows connections with the northeastern corner of the Carpathian basin (Svoboda 1991; ŠKrdLA, Plch 1993). Mohelno-Plevovce KSA and KSB lithic assemblages (ŠKRDLA et al. 2016) together yielded 19.7\% TC flint, and the rest of the material was regionally available, such as the rock crystal and Krumlovsky les chert. The radiolarite, $0.9 \%$ of the assemblage, probably derived from the Danube gravels south from the site, but the White Carpathians and the Bakony Mountain in the Transdanubian range was also mentioned as a source of a few items. The obsidian is the farthest raw material in this assemblage.

This dataset illustrated that LGM hunters exploited regional raw material sources. This was one of the character noticed by SvoBodA and NovÁk (2004) to separate LGM industries from the preceding and the later cultures. The chief use of Middle Danube basin raw materials may refer to that the main foraging zone was located south of the Carpathians and the Sudetes, east-west direction along the Middle Danube basin. Eventually, the mobility in LGM could have been less intense and shorter ranged than in the preceding period. 
Beyond the lithic raw material provenience, further technological data from the LGM period, which is comparable with what has been obtained from the Hungarian assemblages is sparse. Flake production seems to be more pronounced for the tool kits at Kašov I upper layer (BÁNESZ et al. 1991), Stránská skála IV (Svoboda 1991; ŠKrdLA, PlCH 1993), and throughout the sequence of Grubgraben (Montet-White 1990). Plain platforms are however not dominant. Mohelno Plevovce KSA and KSB assemblages have small flake cores and proper blade production is missing (ŠKRDLA et al. 2016). Small blades and bladelets were produced from single platform cores, and flake production seems to make up half of the debitage process. This industry was found similar to Ságvár (ŠKRDLA et al. 2016), and the presented lithic technology indeed seems similar.

\section{The Late Epigravettian}

Among the few LE assemblages in Hungary, the Budapest-Csillaghegy material can be mentioned here, where TC material makes up $72.5 \%$ of the assemblage,but the armature typology does not support LE affiliation. If the prediction that Megyaszó (Dobosi, Simán 1996) could be re-dated to LE is correct (Lengyel 2016), then this would be the only LE industry with $2.9 \%$ of TC material and the dominance of local and regional materials.

Late Epigravettian comparable materials are also few outside Hungary. The westernmost occurrence of LE hunters is Sowin 7 in Silesia (WIŚNIEWSKI et al. 2017). The lithic assemblage of this site exploited solely local raw material sources available right at the location of the site. Brno-Štýrice III lithic assemblage consists of regional and TC material in almost equal share (Nerudoví, Neruda 2014). Targowisko 10 used local materials the most (56.9\%), then regional sources, and obsidian accounts for $3 \%$, which is the greatest portion of Carpathian basin material ever in the Palaeolithic archaeological record of Poland (WILCZYŃSKI 2009).

The proportion of the TC material in LE assemblages in the Middle Danube basin is greater than in the LGM. In the case of Polish and Prut-Dniester sites, again local and regional lithic raw material source exploitation is prevalent, but this is the first time when obsidian left the Carpathian basin northward in a greater amount.

Further details of lithic technology from Targowisko 10 and Sowin 7 are the unidirectional blade knapping method, soft hammer technique and plain platform preparation (WILCZYŃSKI 2009; WIŚNIEWSKI et al. 2012).

\section{CONCLUSION}

An important feature of the studied periods is how raw material sources were exploited and what they mean in the sense of hunter-gatherer subsistence. Most Late Gravettian sites are located near raw material sources (i.e. within $10 \mathrm{~km}$ ): KrakówSpadzista, Petřkovice I, Trenčianske Bohuslavice, Arka, Bodrogkeresztúr and Moravany 
sites; a few are found at localities where lithic sources are available on regional level (Milovice I, Sajószentpéter and Hidasnémeti); and it is exceptional when most of the lithics were retrieved from distant sources (Willendorf II layer 9 and Moravany-Banka). The distribution of a considerable amount of TC material over the territory of the Western Carpathians in LG times (KozŁowSKI 2013) likely marks the range of a hunter-gatherer foraging zone (GOODYEAR 1979). This archaeological record suggests that LG site location choice, besides the necessary food availability, was also based upon the closeness of the lithic source. The co-occurrence of domestic tools and armatures at every LG site shows both domestic and hunting, activity at the sites, which is rather an emblem of residential bases (BINFORD 1980). Altogether, this illustrates that LG hunter-gatherers could have been residentially mobile (BINFORD 1979; KeLLY 1983).

The Váh valley record shows that the northernmost site in the Carpathian basin with abundant settlement remains is Trenčianske Bohuslavice. All other sites are located southward. If we consider LG hunters following the prey (PRYOR 2008), which might be supported by the changes of ${ }^{86} \mathrm{Sr} /{ }^{87} \mathrm{Sr}$ ratio through a reindeer molar enamel in the Trenčianske Bohuslavice fauna (VLAČIKY et al. 2013), than any valley passing from Moravia to Trenčianske Bohuslavice could have been one of the migratory routes of reindeers. If we pair this with the ratio of TC material in LG sites within the Carpathian Basin, we see that Váh valley sites more often have a greater ratio of TC material than sites located inward the Carpathian basin. Studies on the peopling of North America (Graf, Goebel 2009) demonstrated that the first entry to a new land brings lithic raw material from the former foraging area and the new camps contain mostly remote materials. Exploring the new land identify local sources, thus the distant lithic component erodes out from the tool kit only if the former territory is not visited again. In the frame of this theory, it can be proposed that the greater ratio of TC material at Váh valley sites marks the first major camping location within the Carpathian basin. Similarly to the Váh valley sites, Grub-Kranawetberg in Lower Austria, located on the western side of the Lesser Carpathians in river Morava valley, showed that the lower occupation dated to the same period as the upper layer consists of remote Cretaceous flint material and the upper layer already is dominated by radiolarite from the nearby White Carpathians (ANTL-WeISER et al. 2010). This superposition could have been formed shortly after the first settlement, possibly during foraging arrays to the eastern side of the White Carpathians. Similar first base camps from possibly eastern routes across the Carpathians could have been Kašov I lower layer, Arka and Bodrogkeresztúr, where relatively a significant amount of TC material was left at the abundant local lithic sources.

The raw material circulation in Late Gravettian times, however, was unbalanced between the northern and the southern sides of the Western Carpathians. While TC materials flowed into the Carpathian basin, the raw materials of the Carpathian basin hardly travelled beyond the Carpathians. The very few obsidians and radiolarite are the sole markers of the mobility range of LG hunters. This raw material pattern can be due to that TC materials were more important for managing the mobile toolkit, since TC flint nodules usually provide better access to quality in greater quantity than 
the limnic silicites and better options to obtain more blades in greater size with low rate of knapping accidents (LENGYEL 2013). And large flint blades might have fulfilled better the task of a mobile lithic gear (Morrow 1996; Lengyel, Chu 2016). In LG, the only considerable amount of obsidian outside the Tokaj Mountains was found in the Trenčianske Bohuslavice assemblage (ŽAÁr 2007), which eventually did not leave the Carpathian basin towards Moravia, in contrast with the radiolarite.

In the EE period, due to the low integrity or lack of radiocarbon dates, there is no compelling evidence for settlements north of the Carpathians and the Sudetes. Thus, the human foraging territory seems to have been restricted to Moravia, Lower Austria and Carpathian basin during LGM (LeNGyel 2014a). Among EE sites Grubgraben layer 3 and 4 (Montet-White 1990) and Mohelno-Plevovce KSA-KSB (ŠKRDLA et al. 2016) are the sole ones with a high percent of erratic flint, which eventually could have been collected from northern Moravia alike the case of Petŕkovice I LG site (PřICHYSTAL 2008). Towards the Carpathian basin, TC material drastically decreases in the assemblages, and the highest ratio, 6\%, was reported from Mogyorósbánya (DoBosi 2016), which might have originated in Moravian sources. Most LGM sites are not located near lithic raw material sources (ie within $10 \mathrm{~km}$ ), and their assemblages are characterized with regionally available tool stones. The absence of northward mobility was found related with the effect of the Eurasian Ice Sheet advance between 21 and $17 \mathrm{ka}$ BP (Lengyel 2014a). The Carpathian basin could have become a refugee area that provided sufficient resources for surviving near the edge of the Eurasian Ice Sheet. Besides the hints from the lithic raw material provenience and the lithic tool types, EE hunter-gatherers might have established residential bases with abundant site furniture, such as the stone structures in Grubgraben and Mohelno-Plevovce, and the huts at Ságvár, thus, similarly to LG hunters, they also might have been residentially mobile, but on a shorter scale with less frequency.

The LE period raw material circulation was found different from what characterizes the LG and EE. The TC material proportion increased again up to $40 \%$ in Moravia (Brno-Štýrice III) and in the Carpathian basin over 70\%. The farther the location of the site from the TC materials source, the greater the percent of the TC material. The absence of local material and the dwindled frequency of regional material in the Carpathian basin and the high ratio of armature refers to that LE hunter-gatherers equipped themselves for long range mobility and established short term hunting camps (HISCOCK 1994, 2005; Ellis 1997; Elston, Brantigham 2002; Yaroshevich 2006; Robertson et al. 2009; YAroshevich et al. 2010; Hiscock et al. 2011; LengYel 2014b). This theory implies very quick movement across the land and the Carpathian basin could not have been populated as much as it was during the LGM period (VERPOORTE 2004). The fact that this is the first time in the Paleolithic when the obsidian appear in "greater" amount (3\%) over the arch of the Carpathians at Targowisko 10 site, also supports this theory.

The rest of the lithic technology in most cases consisted of blade production. The sole blade technological element that would allow predicting the age of an assemblage is the length, according to which EE blades are shorter than others. On the other hand, blade length was found to be affected by the properties of the raw material. The 
increased flake production on the expense of the blades also seems to be an EE feature. The highly standardized blade production in LE sites is in relation with the long distances mobility and consequently logistical issues (LENGYeL 2014b; LeNGYeL, CHU 2016). The most frequent blade knapping modality, the unipolar debitage, certainly is the simplest way to produce blades. In striking contrast with the typology of the MUP and LUP, which is apt to differentiate the three archaeological cultures studied here (LENGYEL 2016), the blade debitage modalities seem to have been applied flexibly to achieve the desired products with the simplest method adjusted to the raw material properties. This means that none of the hunter-gatherer groups were determined by specific traditions but to maximize efficiency of the production.

ACKNOWLEDGMENT. I am grateful to the staff at the Archaeological Repository of the Hungarian National Museum for providing the facilities to accomplish the study of lithic artifacts. This project has received funding from the European Union's Horizon 2020 research and innovation programme under the Marie Skłodowska-Curie grant agreement No 665778, and financed by the National Science Centre, Poland, agree-

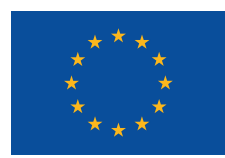
ment No. UMO-2016/23/P/HS3/04034.

\section{REFERENCES}

ANDREFSKY W. Jr., 1994a. The geological occurrence of lithic material and stone tool production strategies. Geoarchaeology: An International Journal 9(5): 375-391.

ANDREFSKY W., 1994b. Raw-material availability and the organization of technology. American Antiquity 59(1): 21-34.

ANDREFSKY W. Jr., 2009. The analysis of stone tool procurement, production, and maintenance. Journal of Archaeological Research 17(1): 65-103.

Antl-Weiser W., Fladerer F. A., Nigst Ph. R., Verpoorte A., 2010. Grub/Kranawetberg (Lower Austria) - Insights into a Gravettian micro-region in Eastern Austria. In: Neugebauer-Maresch Ch., Owen L., (Eds) New aspects of the Central and Eastern European Upper palaeolithic - methods, chronology, technology and subsistence. MPK 72: 231-245.

Bánesz L., Hromada J., Desbrosse R., Margerand I., Kozlowski J. K., Sobczyk K., Pawlikowski M., 1992. Le site de plein air du Paléolithique supérieur de Kasov 1 en Slovaquie orientale. Slovenská Archeológia 40(1): 5-27.

BÁRTA J., 1988. Trenčianske Bohuslavice un habitat gravettien en Slovaquie occidentale. L'Anthropologie 92(4): 173-182.

BINFORD L. R., 1979. Organization and formation processes: looking at curated technologies. Journal of Anthropological Research 35(3): 255-273.

Binford L. R., 1980. Willow Smoke and Dogs' Tails: Hunter-Gatherer Settlement Systems and Archaeological Site Formation. American Antiquity 45(1): 4-20.

BINFORD L. R., 1982. The archaeology of place. Journal of Anthropological Archaeology 1: 5-31.

Binford L. R., 1990. Mobility, Housing, and Environment: A Comparative Study. Journal of Anthropological Research 46(2): 119-152.

Biró K. T., Dobosi V. T., 1991. Lithotheca - The comparative raw material collection of the Hungarian National Museum. Magyar Nemzeti Múzeum, Budapest. 
Biró K. T., Dobosi V. T., SchléDer Z., 2000. Lithitheca - The comparative raw material collection of the Hungarian National Museum. Magyar Nemzeti Múzeum, Budapest.

Boulton G.S., Dongelmans P., Punkari M., Broadgate M., 2004. Evidence of European ice sheet fluctuation during the last glacial cycle. In: Ehlers J., Gibbard P.L, (Eds) Quaternary Glaciations. Extent and Chronology. Part I: Europe. Elsevier, Amsterdam: 441-460.

Bösken J., Sümegi P., Zeeden C., Klasen N., Gulyás S., Lehmkuhl F., 2018. Investigating the last glacial Gravettian site 'Ságvár Lyukas Hill' (Hungary) and its paleoenvironmental and geochronological context using a multi-proxy approach. Palaeogeography, Palaeoclimatology, Palaeoecology 509: 77-90.

Bradák B, Kiss K, Barta G, Varga Gy, Szeberényi J, Józsa S, Novothny Á, Kovács J, Markó A, MÉSzÁros E, Szalai Z., 2014. Different paleoenvironments of Late Pleistocene age identified in Veröce outcrop, Hungary: preliminary results. Quaternary International 319: 119-136.

Clark P. U., Dyke A. S., Shakun J. D., Carlson A. E., Clark J., Wohlfarth B., Mitrovica J. X., Hostetler S. W., Mccabe A. M., 2009. The Last Glacial Maximum. Science 325: 710-714.

Csalogovits J., GaÁl I., Hollendonner F., Hillebrand J., 1931 Az 1931. évi ságvári ásatások eredménye. Archaeológiai Értesítő 45: 240-247.

Cyrek K., Nadachowski A., Madeyska T., Bocheński Z., Tomek T., Wojtal P., Miękina B., Lipecki G., Garapich A., Rzebik-Kowalska B., Stworzewicz E., Wolsan M., Godawa J., Kościów R., Fostowicz-Frelik Ł., Szyndlar Z., 2000. Excavation in the Deszczowa Cave (Kroczyckie Rocks, Częstochowa Upland, Central Poland). Folia Quaternaria 71: 5-84.

Davies W., Hedges R., 2008-2009. Dating a Type Site: Fitting Szeleta Cave into Its Regional Chronometric Context. Praehistoria 9-10: 35-45.

Demars P.-Y., Laurent P., 1992. Types d'outils lithiques du Paléolithique supérieur en Europe. Cahiers du Quaternaire No7. Presses du CNRS, Paris.

Demidenko Y. E., Škrdla P., Rios-Garaizar J., 2018. European perspectives of the East European LGM Epi-Aurignacian with Sagaidak-Muralovka-type microliths. In: Valde-Nowak P., Sobczyk K., Nowak M., Zralka J., (Eds), Multas per gentes et multa per saecula amici magistro et collegae suo Ioanni Christopho Kozłowski dedicant. Krakow: Institute of Archaeology, Jagiellonian University: 85-92.

Doвosi V. T., 1974. Adatok a Bodrog-völgy őskőkorához. Folia Archaeologica 25: 9-32.

Dobosi V. T., 2000a. Upper Palaeolithic research in Hungary - a situation report from 2000. Praehistoria 1: $149-159$.

Dobosi V. T., (ed.) 2000b. Bodrogkeresztúr-Henye (NE Hungary), Upper Palaeolithic site. Hungarian National Museum, Budapest.

Dobosi V. T., 2001. Antecedents: Upper Paleolithic in the Jászság region. In: Kertész R., Makkay J., (Eds) From the Mesolithic to the Neolithic. Archaeolingua, Budapest: 177-191.

Dobosi V. T., 2004. After the golden age (Hungary between 20 and 16 ka BP). In: Dewez M., Noiret P., Teheux E., (Eds) The Upper Palaeolithic. General Sessions and Posters. Acts of the XIVth UISPP Congress, University of Liege, 2-8 September 2001, BAR International Series 1240, Oxford: 153-168.

Doвоsi V. T., 2006. Gravetti lelöhelyek Pilismarót környékén. Folia Archaeologica 52: 21-48.

Doвosi V. T., 2009a. A hazai felső paleolitikum vázlata. Tisicum 19: 13-27.

Dobosi V. T., 2009b. Constancy and Change in Upper Palaeolithic, Hungary. In: Djindjian F., Kozłowski J., Bicho N., (Eds), Le concept de territoires dans le Paléolithique supérieur européen. 15th International Congress of Prehistoric and Protohistoric Sciences (15th: 2006: Lisbon, Portugal), Proceedings of the XV World Congress (Lisbon, 4-9 September 2006), BAR International Series 1938, Archaeopress, Oxford: 123-133.

Dobosi V. T., 2009c. Filling the void: Lithic raw material utilization during the Hungarian Gravettian. In: Adams B., Blades B. S., (Eds) Lithic Materials and Paleolithic Societies. Oxford: 116-126. 
Doвоsı V. T., 2014. A Dunakanyar felső paleolitikuma. Archaeológiai Értesítő 139: 7-33.

Doвosi V. T., 2016. Tradition and modernity in the lithic assemblage of Mogyorósbánya Late Palaeolithic site. Acta Archaeologica Academiae Scientiarum Hungaricae 67: 5-30.

Dobosi V. T., Kövecses-VArga E., 1991. Upper Palaeolithic Site at Esztergom-Gyurgyalag. Acta Archaeologica Academiae Scientiarum Hungaricae 43: 233-255.

Dobosi V. T., Simán K., 1996, New Upper Paleolitic Site at Megyaszó-Szelestető. Communicationes Archaeologicae Hungariae 1996: 5-22.

Doвosi V. T., Simán K., 2003. Hont-Parassa III Orgonás, Upper Palaeolithic Settlement. Communicationes Archaeologicae Hungariae 2003: 15-29.

DoBosi V. T., SzÁNTó Zs., 2003. A gravetti időszak hagyományos és radiokarbon koradatai. Archaeologiai Értesítő 128: 5-16.

Doвоsi V. T., Vörös I. 1987. The Pilisszántó I. Rock-shelter revision. Folia Archaeologica 38: 7-64.

Dobosi V. T., Jungbert B., Ringer Á., Vörös I., 1988. Palaeolithic settlement in Nadap. Folia Archaeologica 39: 13-40.

Dobosi V., KÖHegYi M., Krolopp E., Vörös I., BíRó K. T., 1989. Felsőpaleolit telep Madaras-Téglavetőben, ásatások 1966-1974. Cumania 11: 9-66.

ELLIS C. J., 1997. Factors influencing the use of stone projectile tips. An ethnographic perspective. In: Knecht H., (Ed.) Projectile Technology, Plenum Press, New York: 37-74.

Elston R. G., Brantingham P. J., 2002. Microlithic Technology in Northern Asia: A Risk-Minimizing Strategy of the Late Paleolithic and Early Holocene. In: Elston G. R., Kuhn S. L. (Eds) Thinking Small: Global Perspectives on Microlithization Archeological Papers of the American Anthropological Association 12. Arlington, Virginia: 103-116.

FÉBlot-Augustins J., 1997. La circulation des matieres premieres au paléolithique. Synthese des données perspectives comportementales. Tome I-II. ERAUL 75. Liège.

GÁBORI M., 1959. A ságvgári paleolitikus telep újabb ásatásának eredményei. Archaeológiai Értesítő 86: 3-19.

Gábori M., 1965. Der Zweite Palaolithische Hausgrundriss von Ságvár. Acta Archaeologica Academiae Scientiarum Hungaricae 17: 111-127.

Gábori-Csánk V., 1970. C-14 dates of the Hungarian Palaeolithic. Acta Archaeologica Academiae Scientiarum Hungaricae 22: 3-11.

GÁBori-CsÁnK V., 1978. Une oscillation climatique a la fin du Würm en Hongrie. Acta Archaeologica Academiae Scientiarum Hungaricae 30: 3-11.

Gábori-Csánk V., 1986. Spuren des Jung paläolithikums in Budapest. Acta Archaeologica Academiae Scientiarum Hungaricae 38: 3-12.

GÁBORI M., GÁBORI-CsáNK V., 1957. Études archéologiques et stratigraphiques dans les stations de loess paléolithiques de Hongrie. Acta Archaeologica Academiae Scientiarum Hungaricae 8: 3-117.

Goodyear A.C., 1979. A hypothesis for the use of cryptocrystalline raw materials among paleo-indian groups of North America. Research Manuscript Series. Book 127.

Graf K. E. Goebel T., 2009. Upper Paleolithic Toolstone Procurement and Selection Across Beringia. In: Adams B., Blades B. S., (Eds) Lithic Materials and Paleolithic Societies. Oxford: 54-77.

Grosswald M. G., 1980. Late Weichselian ice sheet of Northern Eurasia. Quaternary Research 13(1): $1-32$.

HäNDEL M., 2017. The stratigraphy of the Gravettian sites at Krems. Quartär 64: 129-155.

Haynes C. V., Damon P. E., Grey D. C., 1966. Arizona Radiocarbon dates VI. Radiocarbon 8: 1-21. 
HERTELENDi E., 1991. Radiocarbon dating of a wood sample from an excavation near Esztergom-Gyurgyalag. Acta Archaeologica Academiae Scientiarum Hungaricae 43: 271.

Hiscock P., 1994. Technological Responses to Risk in Holocene Australia. Journal of World Prehistory 8(3): 267-292.

Hiscock P., 2005. Blunt and to the Point: Changing Technological Strategies in Holocene Australia. In: Lilley I., (Ed.) Archaeology of Oceania: Australia and the Pacific Islands, Blackwell: 69-95.

Hiscock P., Clarkson C., Mackay A., 2011. Big debates over little tools: ongoing disputes over microliths on three continents. World Archaeology 43(4): 653-664.

Hughes P. D, Gibbard P. D, Ehlers J., 2013. Timing of glaciation during the last glacial cycle: evaluating the concept of a global 'Last Glacial Maximum' (LGM). Earth Science Reviews 125: 171-198.

Hughes A. L. C., Gyllencreutz R., Lohne Ř. S., Mangerud J., Svendsen J. I., 2016. The last Eurasian ice sheets - a chronological database and time-slice reconstruction, DATED-1. Boreas 45: 1-45.

Inizan M.-L., Reduron-Ballinger M., Roche H., Tixier J., 1999. Technology and Terminology of Knapped Stone. Meudon: CREP, Nanterre.

KAmINSKÁ L., 2016. Gravettian and Epigravettian lithics in Slovakia. Quaternary International 406(A): $144-165$.

Kaminská L', KozŁowski J.K., 2011. Nitra I-Čermáň V Rámci Štruktúry Osídlenia Gravettienskej Kultúry Na Slovensku. Slovenská Archeológia 41(1): 1-85.

KeLLY R. L., 1983. Hunter-Gatherer Mobility Strategies. Journal of Anthropological Research 39(3): 277-306.

KeLLy R. L., 2013. The lifeways of hunter-gatherers. The foraging spectrum. Cambridge University Press, Cambridge.

Kormos T., Lambrecht K., 1915. A pilisszántói kőfülke. Tanulmányok a postglaciális kor geológiája, ősipara és faunája köréböl. Földtani Intézet Évkönyve 23: 307-498.

Kozıowski J. K., 1979. La fin des temps glaciaires dans le bassin du Danube moyen et inferéeur. In: de Sonneville-Bordes D., (Ed.) La fin des temps glaciaires en Europe, CNRS: 821-835.

Kozıowski J. K., 1996. The Danubian Gravettian as seen from the northern perspective. In: Svoboda J., (Ed.) Palaeolithic in the Middle Danube region, Archeologický ústav AV ČR, Brno: 11-22.

KozŁowski, J. K., (Ed.) 1998. Complex of Upper Palaeolithic sites near Moravany, Western Slovakia, Vol. 2, Moravany-Lopata II (excavations 1993-1996), Kraków: Jagellonian University, Institute of Archaeology.

Kozıowski J. K., (Ed.) 2000. Complex of Upper Palaeolithic sites near Moravany, Western Slovakia, Vol. 3, Late Gravettien shouldered points horizon sites in the Moravany - Banka area, Nitra.

Kozıowski J. K., 2008. The shouldered point horizon and the impact of the LGM on human settlement distribution in Europe. In: Svoboda J., (Ed.) Petřkovice: on shouldered points and female figurines, The Dolní Věstonice Studies Vol 15, Institute of Archaeology at Brno, Academy of Sciences of the Czech Republic, Brno: 181-192.

Kozıowski J. K., 2013. Raw materials procurement in the Late Gravettian of the Carpathian Basin. In: Mester Z., (Ed.) The lithic raw material sources and the interregional human contacts in the Northern Carpathian regions, Polska Akademia Umiejętności, Kraków-Budapest: 63-85.

KozŁowsKi J. K., 2015. The origin of the Gravettian, Quaternary International 359-360: 3-18.

Kozıowski J. K., Sobczyк K., 1987. The Upper Paleolithic site Kraków-Spadzista street C2, Excavations 1980. Prace Archeologiczne 42: 7-68.

Krolopp E., SüMEgi P., 2002. A ságvári lösz rétegsor csigafaunája. Malakológiai Tájékoztató 20: 7-14. 
Laczkó D., GaÁl I., Hollendonner F., Hillebrand J. 1930. A ságvári felső diluviális lösztelep. Archaeológiai Értesítő 44: 213-220.

Lengyel G., 2008-2009. Radiocarbon dates of the "Gravettian Entity" in Hungary. Praehistoria 9-10: $241-263$.

Lengyel G., 2010. An aspect to the re-evaluation of Ságvár (Lyukas-domb) Upper Palaeolithic site. Folia Archaeologica 54: 25-37.

Lengyel G., 2011. The pebble, the block and the tabular. Lithic raw material use at Ságvár Lyukas-domb Upper Palaeolithic site. In: Biró K. T., Markó A., (Eds) Emlékkönyv Violának, Tanulmányok T. Dobosi Viola tiszteletére. (Papers in Honour of Viola T. Dobosi) Magyar Nemzeti Múzeum, Budapest: 93-102.

Lengyel G., 2013. Knapping experiments on lithic raw materials of the Early Gravettian in Hungary. In: Mester Z., (Ed.) The lithic raw material sources and interregional human contacts in the Northern Carpathian regions. Polish Academy of Arts and Sciences - Institute of Archaeological Sciences of the Eötvös Loránd University, Kraków-Budapest: 39-51.

LenGYel G., 2014a. Distant connection changes from the Early Gravettian to the Epigravettian in Hungary. In: Otte M., le Brun-Ricalens F., (Eds) Modes de contacts et de déplacements au Paléolithique eurasiatique: Modes of contact and mobility during the Eurasian Palaeolithic ERAUL 140 - ArhéoLogiques 5. Université de Liege, Liege-Luxembourg: 331-347.

Lengyel G., 2014b. Backed tool technology at Esztergom-Gyurgyalag Epigravettian site in Hungary. In: Biró K.T., Markó A., Bajnok K.P., (Eds) Aeolian Scripts: New Ideas On The Lithic World, Studies In Honour Of Viola T. Dobosi. Budapest: Magyar Nemzeti Múzeum: 121-129.

LENGYeL G., 2015. Lithic raw material procurement at Bodrogkeresztúr-Henye Gravettian site, northeast Hungary. Quaternary International 359-360: 292-303.

Lengyel G., 2016. Reassessing the Middle and Late Upper Palaeolithic in Hungary. Acta Archaeologica Carpathica 51: 47-66.

Lengyel G., Chu W., 2016. Long thin blade production and Late Gravettian hunter-gatherer mobility in Eastern Central Europe. Quaternary International 406(A): 166-173.

LeNGYeL G., WiLCZYŃSKI J., 2018. The Gravettian and the Epigravettian chronology in eastern central Europe: a comment on Bösken et al. 2017. Palaeogeography, Palaeoclimatology, Palaeoecology 506: 265-269.

Lengyel G., Mester Z., Szolyák P., 2016. The Late Gravettian and Szeleta Cave, northeast Hungary. Quaternary International 406(A): 174-183.

Lorenc M., 2006. Radiocarbon dating of some Late Pleistocene faunal assemblages in caves in Poland. Acta zoologica cracoviensia, 49A(1-2): 41-61.

MaIER A., 2015. The Central European Magdalenian. Regional Diversity and Internal Variability, Springer.

Markó A., 2007. The Upper Palaeolithic site at Szob. Folia Archaeologica 53: 7-22.

Markó A., 2011. A little puzzle: further studies on the Upper Palaeolithic assemblage of Szob. Folia Archaeologica 54: 9-24.

MARKó A., 2015. Istállóskő revisited: Lithic artefacts and assemblages, sixty years after. Acta Archaeologica Academiae Scientiarum Hungaricae 66: 5-38.

Markó A., 2017. Use of Obsidian During the LGM: Case Studies from the Pebble Gravettian Sites in Hungary. Archeometriai Mühely 14(3): 131-142.

Marks L., 2012. Timing of the Late Vistulian (Weichselian) glacial phases in Poland. Quaternary Science Reviews 44: 81-88.

Mester Z., 2013. The lithic raw material sources and interregional human contacts in the northern Carpathian regions: aims and methodology. In: Mester Z. (Ed.), The lithic raw material sources and 
interregional human contacts in the northern Carpathian regions. Polish Academy of Arts and Sciences, Kraków-Budapest: 9-21.

Montet-White A., (Ed.) 1990. The Epigravettian Site of Grubgraben, Lower Austria: The 1986 and 1987 Excavations, ERAUL 40, l'Université de Liège, Liège.

Moreau L., 2009. Geißenklösterle. Das Gravettien der Schwäbischen Alb im europäischen Kontext, Kerns Verlag, Tübingen.

Moreau L., 2012. Le Gravettien ancien d'Europe centrale revisité: mise au point et perspectives. L'Anthropologie 116: 609-638.

Morrow T. A., 1996. Bigger is Better: Comments on Kuhn's Formal Approach to Mobile Tool Kits. American Antiquity 61(3): 581-590

Nadachowski A., Żarski M., Urbanowski M., Wojtal P., MięKina B., Lipecki G., Ochman K., KraWCZYK M., JaKUBOwski G., TomeK T., 2009. Late Pleistocene environment of the Częstochowa Upland (Poland) reconstructed on the basis of faunistic evidence from archaeological cave sites. Institute of Systematics and Evolution of Animals, Polish Academy of Sciences, Kraków

Nerudoví Z., Neruda P., 2014. Štýřice III (Koněvova St. or Vídeňská St.) - an Epigravettian Site in Brno (Czech Republic). Interdisciplinaria Archaeologica 5: 7-18.

Neugebauer-Maresch C., Einwögerer T., Richter J., Maier A., Hussain S.T., 2016. Kammern-Grubgraben. Neue Erkenntnisse zu den Grabungen 1985-1994. Archaeologia Austriaca 100: 225-254.

Novák M., 2004. Gravettian occupation in the lower layer of Kašov I. In: Svoboda J.A., Sedlácková L., (Eds) The Gravettian Along the Danube. Proceedings of the Mikulov Conference, The Dolní Vestonice Studies, Vol. 11. Brno: 217-242.

NovíK M., 2008. Flint and radiolarite assemblages: technology and typology. In: Svoboda J.A., (Ed.) Petřkovice: On Shouldered Points and Female Figurines, The Dolní Věstonice Studies, Vol 15. Institute of Archaeology at Brno, Academy of Sciences of the Czech Republic, Brno: 70-142.

Oliva M., (Ed.) 2009. Sídliště mamutího lidu, u Milovic pod Pálavou: otázka strukturs mamutími kostmi (Milovice, site of the mammoth people below the Pavlov hills: the question of mammoth bone structures). Studies in Anthropology, Palaeoethnology and Quaternary Geology 27, ns 19, Brno.

Отте M., 1981. Le Gravettien en Europe centrale, Dissertationes Archaeologicae Gandenses XX, 2 vol. Bruges, De Tempel.

Patou-Mathis M., Vercoutère C., Lengyel G., Szolyák P., Mester Z. 2016. New interpretation of the Upper Palaeolithic human occupations at Istállóskő Cave (Bükk Mountains, Hungary). Eurasian Prehistory 13(1-2): 77-90.

Pelegrin J., 2011. Sur les débitages laminaires du Paléolithique supérieur. In: Delpech F., Jaubert J., (Eds) François Bordes et la préhistoire. Actes du colloque international François Bordes, Bordeaux, 22-24 avril 2009, éditions du CTHS, coll. Documents préhistoriques, $n^{\circ} 29$, Bordeaux: 141-152.

PÉCSI M., 1985. Chronostratigraphy of Hungarian loesses and the underlying subaerial formation. In: Pécsi M., (Ed.) Loess and the Quaternary. Chinese and Hungarian case studies. Studies in Geography in Hungary 18. Akadámiai Kiadó, Budapest: 33-49.

Poltowicz-Bobak M., 2012. Observations on the late Magdalenian in Poland. Quaternary International 272-273: 297-307.

Priskin A., 2011. Jászfelsőszentgyörgy-Szúnyogos és - Székes-dűlő felső paleolit lelőhelyek pattintott kőeszköz anyaga (tipológia és nyersanyag felhasználás). Unpublished MA thesis, University of Pécs.

Pryor A.J.E., 2008. Following the fat: food and mobility in the European Upper Palaeolithic 45,000 to 18,000 BP. Archaeological Review from Cambridge 23(2): 161-179.

PřIChystal A., 2008. Lithic raw materials. In: Svoboda J.A., (Ed.) Petřkovice: On Shouldered Points and 
Female Figurines, The Dolní Věstonice Studies, Vol 15. Institute of Archaeology at Brno, Academy of Sciences of the Czech Republic, Brno: 67-69.

PřIChYSTAL A., 2013. Lithic raw materials in prehistoric times of Eastern Central Europe. Masaryk University, Brno.

Ringer Á., Holló Z., 2001. Sajószentpéter Margit-kapu-dűlő, egy felső-paleolit lelőhely a Sajó völgyében, A Herman Ottó Múzeum Évkönyve 40: 63-72.

Ringer Á., Lengyel G., 2008-2009. The Upper Palaeolithic Site at Budapest Corvin-tér, Praehistoria 9-10: 205-211.

Robertson G., Attenbro, V., Hiscock P., 2009. Multiple uses for Australian backed artefacts. Antiquity 83(320): 296-308.

Sнотт M., 1986. Technological Organization and Settlement Mobility: An Ethnographic Examination. Journal of Anthropological Research 42(1): 15-51.

SimÁn K., 1989. Hidasnémeti - Upper Palaeolithic Site in the Hernád valley (Northeast Hungary), Acta Archaeologica Carpathica 28: 5-24.

SimÁn K., 1990 Considerations on the "Szeletian unity". In: Kozłowski J.K., (Ed.) Feuilles de pierre. Les industries á pointes foliacées du Paléolithique supérieur européen, ERAUL 42, Liège, Université de Liège: 189-198.

Sümegi P., Krolopp E., Hertelendi E., 1998. A Ságvár-Lascaux Interstadiális paleoökológiai rekonstrukciója. Közlemények a Debreceni Kossuth Lajos Tudományegyetem Ásvány- és Földtani Intézetéből 71: $165-180$.

SÜMEgi P., RudNER E., DeSZEDA I., 2000. Stratigraphical and palaeoecological investigation of the fossil soil comprising Upper Palaeolithic tools at Bodrogkeresztúr-Henye. In: Dobosi V.T., (Ed.) BodrogkeresztúrHenye (NE Hungary), Upper Palaeolithic site. Hungarian National Museum, Budapest: 217-220.

Stroeven A. P., Hattestrand C., Kleman J., Heyman J., Fabel D., Fredin O., Goodfellow B. W., Harbor J. M., Jansen J. D., Olsen L., Caffee M. W., Fink D., Lundqvist J., Rosqvist G. C., Stromberg B., JANSSON K. N., 2015. Deglaciation of Fennoscandia. Quaternary Science Reviews 147: 91-121.

Svoboda J., 1991. Stránská skála. Výsledky výzkumu v letech 1985-1987. Památky archeologické 82: $5-47$.

Svoboda J., 1996. The Pavlovian: typology and behavior. In: Svoboda J., (Ed.) Palaeolithic in the Middle Danube region, Archeologický ústav AV ČR, Brno: 283-301.

Svoboda J. A., 2007. The Gravettian on the Middle Danube. Paléo 19: 203-220.

Svoboda J., 2016. Settlement units and human fossils. Spatial context, stratigraphies and chronology. In: Svoboda J., (Ed) Dolní Vestonice II. Chronostratigraphy, paleoethnology, paleoanthropology. Th Dolní Vestonice Studies vol. 21: 16-48.

Svoboda J. A., NovÁk M., 2004. Eastern Central Europe after the Upper Pleniglacial: Changing points of observation. Archäologisches Korrespondenzblatt 34: 463-477.

SzoLYÁK P., 2008-2009. New Radiocarbon Data with Stratigraphical, Climatic and Archaeological Contexts to the Palaeolithic Assemblage of the Herman Ottó Cave, Miskolc-Alsóhámor, Northeast Hungary. Praehistoria 9-10: 213-224.

ŠKrdla P., Plch M., 1993. Osídlení epigravettienu v okolí Stránské skály (okr. Brno-město). Archeologické rozhledy 45: 429-435.

Škrdla P., Nejman L., Bartík J., RychtaŘíkova T., Nikolajev P., Eigner J., NÝvltova Fišáková M., NovÁk J., Polanská M., 2016. Mohelno - A terminal Last Glacial Maximum industry with microlithic tools made on carenoidal blanks, Quaternary International 406(A): 184-194. 
Tallavaaraa M., Luotob M., Korhonenc N., Järvinend H., Seppë H., 2015. Human population dynamics in Europe over the Last Glacial Maximum. PNAS 112(27): 8232-8237.

Tixier J., 2012. A method for the study of stone tools. ArchéoLogique 4. Centre National de Recherche Archéologique Musée National d'Histoire et d'Art, Luxemburg.

Tolna-Dobosi V. T., 2001. About Ságvárian: chronological-cultural sketch of the Upper Palaeolithic in Hungary. In: Ginter B., Drobniewicz B., Kazior B., Nowak M., Poltowicz M., (Eds) Problems of the Stone Age in the Old World, Jagellonian University, Institute of Archaeology, Kraków: 195-201.

VARGA I., 1991. Mineralogical analysis of the lithic material from Esztergom-Gyurgyalag. Acta Archaeologica Academaiae Scientiarum Hungariae 43: 267-269.

Verpoorte A., 2004. Eastern Central Europe during the Pleniglacial. Antiquity 78: 257-266.

VERPOORTE A., 2009. Limiting factors on early modern human dispersals: The human biogeography of late Pleniglacial Europe. Quaternary International 201: 77-85.

VÉRTES L., 1955. Neuere Ausgrabungen und paläolithische Funde in der Höhle von Istállóskő. Acta Archaeologica Academiae Scientiarum Hungaricae 5: 111-131.

VÉRTES L., 1962. Ausgrabungen der altsteinzeitlichen Siedlung von Arka 1960-61, Acta Archaeologica Academiae Scientiarum Hungaricae 14: 143-157.

VÉRTES L., 1964-1965. Das Jungpaläolithikum von Arka in Nord-Ungarn. Quartär 15-16: 79-132.

VÉRTES L., 1965. "Lunar Calendar” from the Hungarian Upper Paleolithic. Science 149(3686): 855-856.

VÉRTES L., 1966. The Upper Palaeolithic Site on Mt. Henye at Bodrogkeresztúr, Acta Archaeologica Academiae Scientiarum Hungaricae 18: 3-14.

Vita-Finzi C., Higgs E. S., Sturdy D., Harriss J., Legge A. J., Tippett H., 1970. Prehistoric Economy in the Mount Carmel Area of Palestine: Site Catchment Analysis. Proceeding sof the Prehistoric Society 36: 1-37.

Vlačiky M., Michalík T., NÝvltová FišÁková M., NÝvlt D., Moravcová M., KráLík M., Kovanda J., PéKová K., Přichystal A., Dohnaloví A., 2013. Gravettian occupation of the Beckov Gate in Western Slovakia as viewed from the interdisciplinary research of the Trenčianske Bohuslavice - Pod Tureckom site. Quaternary International 294: 41-60.

Vogel J.C., Waterbolk H.T., 1964. Groningen Radiocarbon Dates V. Radiocarbon 6: 349-369.

Vogel J.C., Waterbolk H.T., 1967. Groningen Radiocarbon Dates VII. Radiocarbon 9: 107-155.

Vörös I., 1982. Faunal remains from the Gravettian reindeer hunter's campsite at Ságvár. Folia Archaeologica 33: 43-71.

Vörös I., 2000. Macro-mammal remains on Hungarian Upper Pleistocene sites. In: Dobosi V. T., (Ed.) Bodrogkeresztúr-Henye (NE Hungary), Upper Palaeolithic site, Hungarian National Museum, Budapest: $186-212$.

ŽAÁR O., 2007. Gravettienska stanice v Trenčianskich Bohuslavicach. Unpublished MA thesis, Filozofická Fakulta, Univerzita Konštantína Filozofa v Nitre. Nitra.

WiLCZYŃSKi J., 2007. The Gravettian and Epigravettian lithic assemblages from Kraków-Spadzista B+B1: dynamic approach to the technology. Folia Quaternaria 77: 37-96.

WiLCZYŃSKi J., 2009. Targowisko - a new Late Glacial site in southern Poland. Eurasian Prehistory 6: 95-118.

WiLCZYŃSKi J., (Ed.) 2015. A Gravettian Site in Southern Poland, Jaksice II. Plosh Academy of Sciences, ISEA PAS, Kraków.

WiLCZYŃSKi J., 2016. Variability of Late Gravettian lithic industries in southern Poland: A case study of the Krakow Spadzista and Jaksice II sites, Quaternary International 406(A): 129-143. 
WilczyŃski J., Wojtal P., Sobieraj D., Sobczyk K., 2015. Kraków Spadzista trench C2: New research and interpretations of Gravettian settlement. Quaternary International 359-360: 96-113.

Wiśniewski A., Furmanek M., Borowski M., KąDZıoŁka K., Rapiński A., Winnicka K., 2012. Lithic raw material and Late Palaeolithic strategies of mobility: a case study from Sowin 7, SW Poland. Anthropologie 50(4): 391-409.

Wiśniewski A., PŁonka T., Jary Z., Lisa L., Traczyk A., Kufel-Diakowska B., Raczyk J., Bajer A., 2015. The early Gravettian in a marginal area: New evidence from SW Poland. Quaternary International 359-360: 131-152.

Wiśniewski A., Poltowicz-Bobak M., Bobak D., Jary Z., Moska P., 2017. The Epigravettian and the Magdalénian in Poland: new chronological data and old problem. Geochronometria 44: 16-29.

Yaroshevich A., 2006. Techno-morphological aspects of microlithic projectile implements: examples from the Levantine Geometric Kebaran and the east European Epigravettian. Archaeology, Ethnology \& Anthropology of Eurasia 4(28): 8-17.

Yaroshevich A., Kaufman D., Nuzhny D., Bar-Yosef O., Weinstein-Evron M., 2010. Design and performance of microlith implemented projectiles during the Middle and the Late Epipaleolithic of the Levant: experimental and archaeological evidence Journal of Archaeological Science 37: 368-388. 